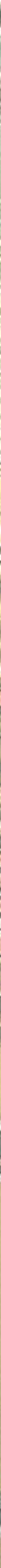
To WaterWave

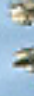




\title{
Variational Approaches \\ To Water Wave Simulations
}

\author{
Elena Gagarina
}

University of Twente 2014 


\section{Composition of the Graduation Committee:}

Chairman and Secretary:

Prof. Dr. P. M. G. Apers University of Twente

Promotors:

Prof. Dr. Ir. J. J. W. van der Vegt University of Twente

Prof. Dr. Ir. O. Bokhove University of Leeds

Members:

Prof. Dr. Ir. E. W. C. van Groesen University of Twente

Prof. Dr. H. J. Zwart University of Twente

Prof. Dr. Ir. R. H. M. Huijsmans Delft University of Technology

Prof. Dr. A. E. P. Veldman University of Groningen

Referee:

Dr. T. Bunnink

MARIN

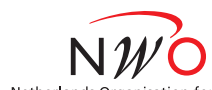

Netherlands Organisation for Scientific Research

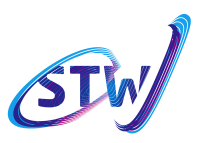

Nieuwe technologie

mogelijk maken
J.M.Burgerscentrum

ISBN: 978-90-36-3754-4

DOI: $10.3990 / 1.9789036537544$

http://dx.doi.org/10.3990/1.9789036537544

Printed by CPI-Wöhrmann Print Service, Zutphen.

Copyright (c) 2014 Elena Gagarina, Enschede, The Netherlands. 


\section{VARIATIONAL APPROACHES TO WATER WAVE SIMULATIONS}

DISSERTATION

to obtain

the degree of doctor the University of Twente, on the authority of the Rector Magnificus,

Prof. Dr. H. Brinksma,

on account of the decision of the Graduation Committee, to be publicly defended

on Friday 3 October 2014 at 12:45

by

Elena Vitalyevna Gagarina

born on 25th of November 1987

in Kiev, USSR 
The dissertation is approved by:

Prof. Dr. Ir. J. J. W. van der Vegt (promotor)

Prof. Dr. Ir. O. Bokhove (promotor) 
To the memory of Sergei Ivanovich Gagarin 


\section{Preface}

The research presented in this dissertation was carried out at the Mathematics of Computational Science (MaCS) group, Department of Applied Mathematics, Faculty of Electrical Engineering, Mathematics and Computer Science pf the University of Twente, The Netherlands.

The work has been a part of the STW project "Complex wave-current interactions in a numerical wave tank" and NWO project "Compatible Mathematical Models for Coastal Hydrodynamics". The support from the Technology Foundation STW, the Netherlands Organization for Scientific Research (NWO), the Maritime Research Institute Netherlands MARIN is gratefully acknowledged.

The project has been associated with the J. M. Burgers Research School for Fluid Dynamics and the Nanotechnology Research institute of the Univeristy of Twente MESA+. 


\section{Contents}

1 Introduction 1

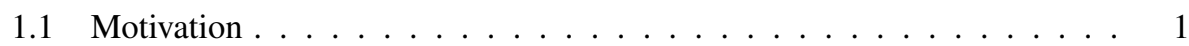

1.2 Objectives . . . . . . . . . . . . . . . . . . . . . 4

1.3 Understanding water waves . . . . . . . . . . . . . . . . . . . . . . . . . . . . .

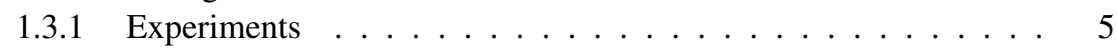

1.4 Modeling water waves $\ldots \ldots \ldots \ldots$

1.4.1 Mathematical wave models . . . . . . . . . . . . . 6

1.4 .2 Numerical methods . . . . . . . . . . . . . . . . . . 7

1.4 .3 Numerical wave tank . . . . . . . . . . . . . . . . . . . . . . . . . . . . .

1.4 .4 Variational dynamics . . . . . . . . . . . . . . . . . . . . . 9

1.5 Outline ............................... 10

2 Horizontal circulation and jumps in Hamiltonian wave models 13

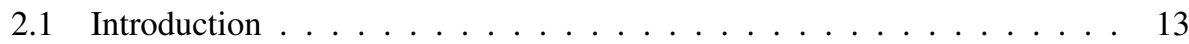

2.2 New water wave model . . . . . . . . . . . . . . . . . . . . . . . . . . . . . . . 15

$2.2 .1 \quad$ Variational principle . . . . . . . . . . . . . . . 15

2.2 .2 Reduction of Hamiltonian dynamics . . . . . . . . . . . . . . 18

2.2 .3 Hamiltonian dynamics of new water wave model . . . . . . . . . 19

2.3 Shallow water and potential flow limits . . . . . . . . . . . . . . . . 22

2.4 Hamiltonian Boussinesq reductions of new model . . . . . . . . . . . . . 23

2.4 .1 Variational Boussinesq model . . . . . . . . . . . . . . . 24

2.4 .2 Green-Naghdi limit . . . . . . . . . . . . . . . . . . . . 26

2.5 Jump conditions for bores . . . . . . . . . . . . . . . . . . . 28

$2.5 .1 \quad$ 1D Jump conditions for shallow water equations . . . . . . . . . 29

2.5 .2 2D Jump conditions for new water wave model . . . . . . . . . . 33

2.5 .3 2D Jump conditions for shallow water equations . . . . . . . . . . . 38

2.5 .4 1D Jump conditions for new water wave model . . . . . . . . . . 39 
2.5.5 1D Jump conditions for variational Boussinesq and Green-Naghdi

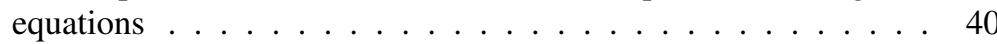

2.6 Conclusions . . . . . . . . . . . . . . . . . . . . . . . . . 40

2.7 Appendix . . . . . . . . . . . . . . . . . . . . . . 42

2.7 .1 Integrals . . . . . . . . . . . . . . . 42

2.7.2 Variational Reynolds' transport theorem . . . . . . . . . . . . . 43

3 Symplectic Time Discontinuous Galerkin Discretizations for Hamiltonian Sys-

tems 49

$3.1 \quad$ Introduction . . . . . . . . . . . . . . . . . . . . . . . . 49

3.2 Dynamics of a Hamiltonian system with one degree of freedom. . . . . . 51

3.3 Variational discontinuous Galerkin time discretization of a Hamiltonian

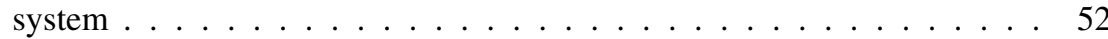

3.3 .1 Discrete functional . . . . . . . . . . . . . 52

3.3 .2 First order variational time discretization . . . . . . . . . . 54

3.3 .3 Second order variational time discretization: Störmer-Verlet . . . 55

3.3 .4 Second order time discretization: Modified symplectic midpoint

3.4 New variational time integrators . . . . . . . . . . . . . 60

3.4.1 Explicit second order accurate time integration scheme . . . . . . 61

3.4.2 Third order scheme . . . . . . . . . . . . . 62

3.5 Applications to non-autonomous systems . . . . . . . . . . . . . . 69

3.5.1 Damped oscillator . . . . . . . . . . . . . 71

3.5 .2 Forced oscillator . . . . . . . . . . . . . . . . 74

3.6 Conclusions . . . . . . . . . . . . . . . . . . . . . . 78

3.7 Appendix . . . . . . . . . . . . . . . . . . 78

3.7 .1 Continuum harmonic oscillator . . . . . . . . . . . . 78

3.7 .2 Störmer-Verlet scheme . . . . . . . . . . . . . . 80

3.7.3 New explicit second order variational time integration scheme . . 82

3.7 .4 Extended continuum system . . . . . . . . . . . 89

4 Variational space-time (dis)continuous Galerkin method for nonlinear free $\begin{array}{ll}\text { surface water waves } & 93\end{array}$

$4.1 \quad$ Introduction . . . . . . . . . . . . . . . . . . . . . . . . . . . 94

$4.2 \quad$ Variational Description of Nonlinear Inviscid Water Waves . . . . . . . . 96

4.3 Variational Finite Element Formulation . . . . . . . . . . . . . . . . . 99

4.3 .1 Tessellation . . . . . . . . . . . . . . . . . 99

4.3.2 Function spaces and approximations . . . . . . . . . . . . . 100

4.3.3 Space-plus-time formulation . . . . . . . . . . . . . . . 102

4.3 .4 Dynamics . . . . . . . . . . . . . . . . . . . . . . . . . . . . . . . . . . . . . . . . . . . . .

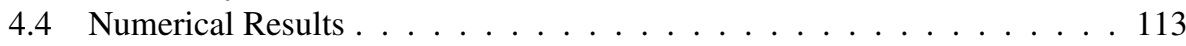

4.4 .1 Verification . . . . . . . . . . . . . . . . . . . . . . . . . . . . . . . . . .

4.4 .2 Validation against laboratory data $\ldots \ldots \ldots . . \ldots 115$ 
4.5 Application of new third order symplectic time integrator . . . . . . . . . 123 4.5 .1 Nonlinear potential flow water waves . . . . . . . . . . . 127

4.6 Conclusions . . . . . . . . . . . . . . . . . . . 132

4.7 Appendix . . . . . . . . . . . . . . . . . . . 133

4.7 .1 Evaluation of finite element matrices . . . . . . . . . . . . 133

4.7 .2 Variations of the kinetic energy . . . . . . . . . . . . . 134

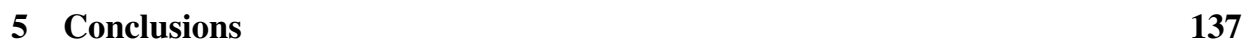

5.1 Recommendations . . . . . . . . . . . . . . . . . . 137

$\begin{array}{ll}\text { Summary } & 139\end{array}$

$\begin{array}{ll}\text { Samenvatting } & 141\end{array}$

\begin{tabular}{ll}
\hline Bibliography & 143
\end{tabular}

\begin{tabular}{ll}
\hline Acknowledgments & 157
\end{tabular} 


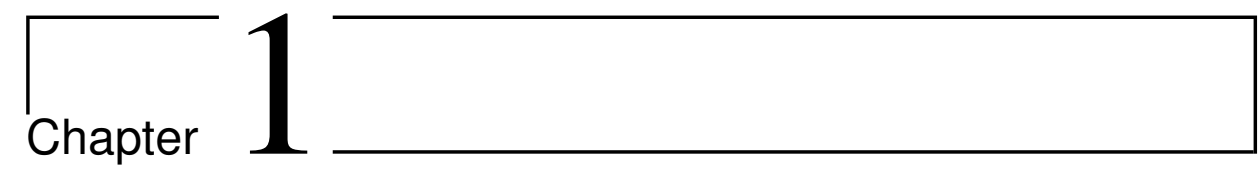

\section{Introduction}

\subsection{Motivation}

A wise man once said that one can observe fire and water forever. The elements are fascinating indeed, what is their secret? Perhaps, the reason is the subtle interplay between life and death. We don't exist without water, but it can also easily kill. Water has always been a crucial part in the progress of mankind. The largest, richest, and most important cities are those that have easy access to a convenient water transportation route. This allows the exchange of goods and knowledge between civilizations. For many centuries explorers and merchants were sacrificing their lives in a battle with nature to explore new shipping routes and distant countries. It is no surprise, that understanding the behavior of water waves became of great importance to humanity. This was also motivated by the many natural disasters that had a great impact on society.

One of the most dangerous events for coastal towns is a tsunami wave. The Santorini eruption and subsequent tsunami are believed to be the cause of the Minoan civilization downfall. The disastrous 1755 Lisbon earthquake and tsunami, Fig. 1.1.a), influenced the mind of many Europeans. For instance, Voltaire, Rousseau, and Kant were trying to comprehend in their works a power so supernatural and unfriendly. An extensive overview of floods, tsunamis and other natural disasters can be found in [117]. These events have also extensively motivated the visual arts, see [84].

The recent tragic events of the Boxing Day Tsunami in 2004 and the 2011 Tohoku Tsunami reveal how unprotected modern society still can be when disaster strikes. Possible ways of protection against disastrous waves, such as dikes, wave breakers, and mangrove forests, are therefore of great importance. A novel way to protect people from a devastating tsunami is an alarm system that is based on accurate and fast methods to simulate wave propagation and run-up in the near coastal zone.

On the open seas tsunamis are hardly noticeable, but severe storms, resulting in large 

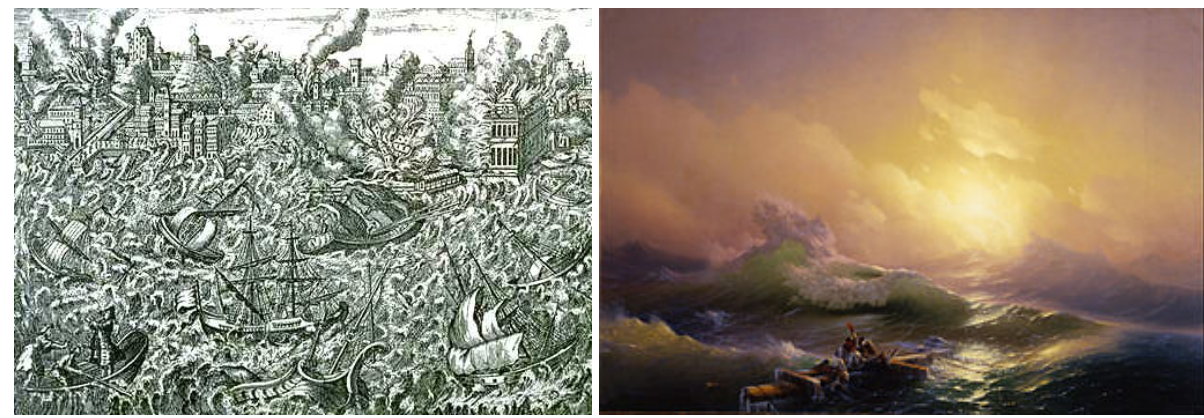

Figure 1.1: (a) A copper engraving showing Lisbon, devastated by the earthquake and subsequent tsunami in 1755. Original in: Museu da Cidade, Lisbon. Source: Wikipedia.org. (b) "The Ninth Wave" - a famous painting of Russian Armenian marinist Ivan Aivazovsky. Collection of the State Russian Museum, St. Petersburg. Source: Wikipedia.org.
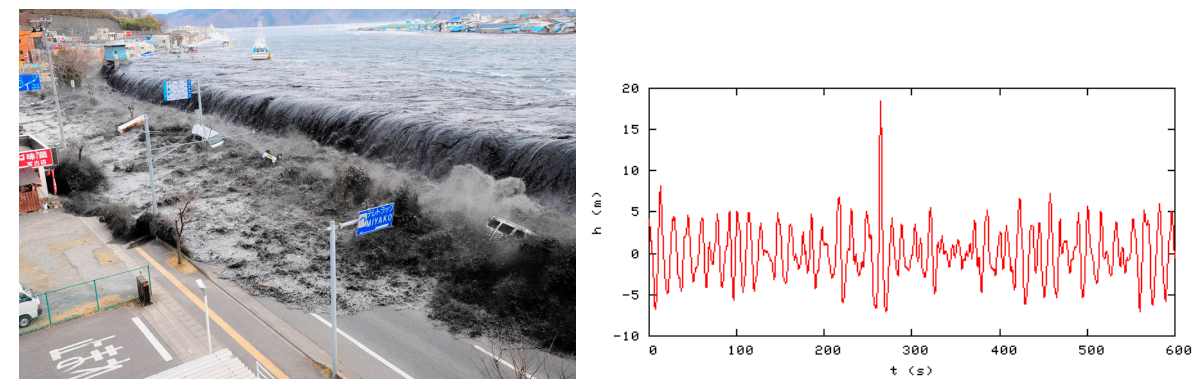

Figure 1.2: (a) Tsunami wave enters Miyako city in Japan in 2011. Authorship: Mainichi Shimbun /Reuters. Source: reuters.com. (b) The Draupner wave or New Year's wave is one of the first measured rogue waves. It occurred at the Draupner platform in the North Sea on 1 January 1995. Source: Wikipedia.org.

and sometimes extreme waves, present a serious danger to ships and offshore structures. Recently, special attention has been given to rare extreme wave events, called freak or rogue waves. In 1978 the German carrier München sank. The investigation into this accident revealed that the ship was most likely damaged by a wave of up to $30 \mathrm{~m}$ height. In 1942 the passenger ship "Queen Mary" was transporting troops from the USA to Europe. Sixteen thousand people were on board when a wave of 28 meters height stroke the ship's side and tilted it by 52 degrees [95]. Those people were just lucky to survive. Nevertheless, the actual causes of ship damages and losses in deep water often remain unclear. Freak waves, which used to be a scary fisherman tale, now are proven to exist [76]. One of the first recorded freak waves is the Draupner wave, see Fig. 1.2 (b), it had a maxi- 
mum height of $25.6 \mathrm{~m}$, which more than twice exceeded the significant wave height. The southern tip of Africa is also a place where extreme waves happen more frequently, see Fig. 1.3 (b). One of the possible mechanisms to explain this is the special kind of wavecurrent interaction occurring in this region due to the Agulhas current, which is opposite to the direction of predominant winds.

In order to understand extreme wave phenomena it is important to define which sea states can result in the occurrence of extreme waves. A second important question is how to minimize the risks from freak waves and severe storms to ships and offshore structures, see Figs. 1.3 and 1.4 (a).
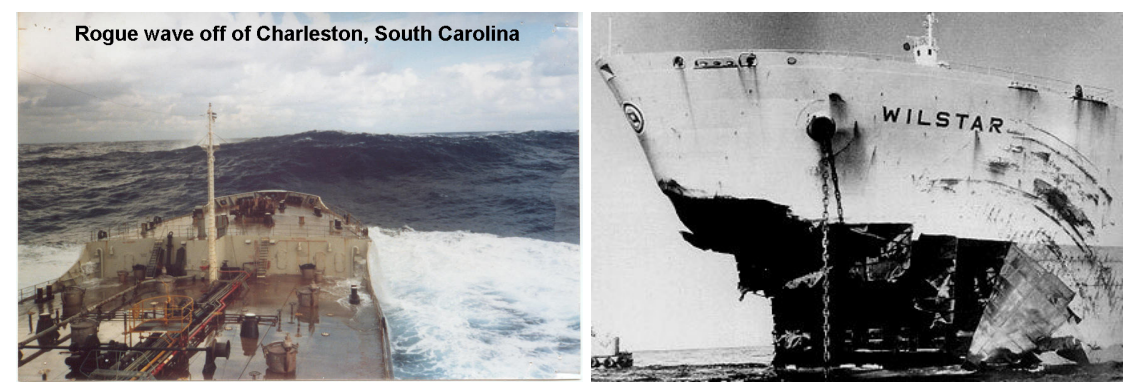

Figure 1.3: (a) Rogue wave estimated at 18 meters moving away from a ship after crashing into it a short time earlier. Gulf Stream off Charleston, South Carolina. Source: NOAA. (b) Norwegian tanker "Wilstar" survived a freak wave encounter in 1974 near the east coast of South Africa. Source: Photo collection of the European Space Agency with credits to H. Gunther and W. Rosenthal.

These questions are especially important for the design of new ships and oil platforms. In particular, this requires knowledge on the wave-floating structure interaction, since insurance companies want to make sure that the structure meets the safety requirements. The dramatic experience of Hurricanes Ivan, Rita and Katrina stimulated extensive research in extreme waves and has resulted in changes in construction requirements for offshore structures. Several extensive studies contributed to these new regulations. For instance, the project Extreme Seas [134] studied wave conditions in the northern hemisphere and consequently, proposed new safety criteria. Also, the study [12] predicts increasingly extreme wave heights in the coming years due to climate change and also gives recommendations for an update of construction requirements in tanker design.

Another aspect of (extreme) wave-structure interaction is the protection of cities. Wave surges, induced by hurricanes, might damage coastal cities, as is, unfortunately, observed over and over again, Fig. 1.4 b). Even without extreme events the knowledge of wave impact on a structure is important. For example, the streets of fragile Venice are suffering from ship-induced waves, which makes it necessary to restrict the boat speed. 

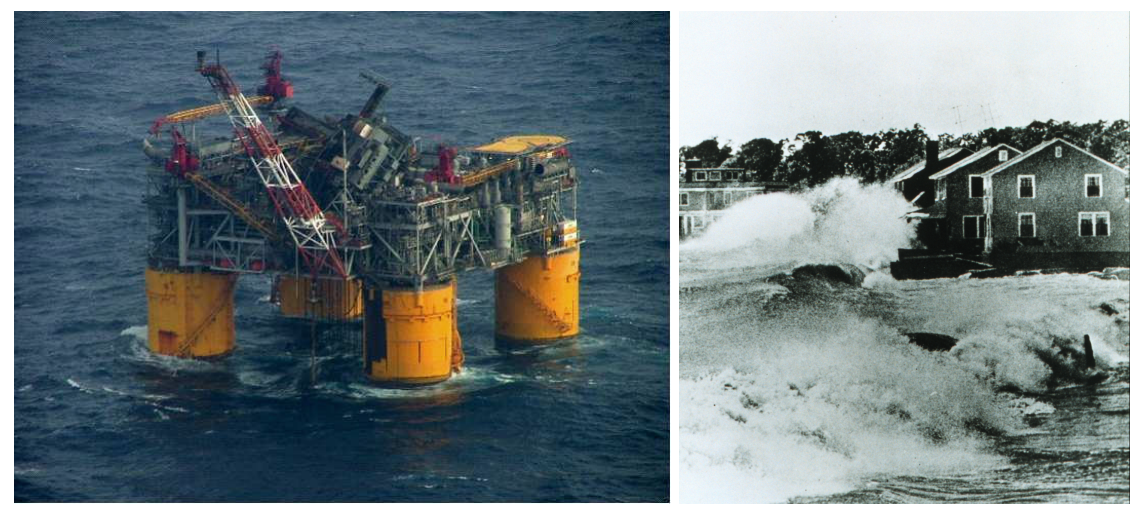

Figure 1.4: (a) Mars oil platform damaged by Hurricane Katrina in 2005. Source: Wikipedia.org. (b) Hurricane Carol induced huge waves in 1954 that destroyed hundreds of houses in Connecticut. Source: NOAA.

\subsection{Objectives}

Given the importance of various types of water waves to coastal regions and engineering, we aim to study the mathematical aspects of free surface flows in this thesis. There are many mathematical models describing water waves under different assumptions. We can study those models analytically or choose a numerical method. One of the tasks we will concentrate on is the construction of a numerical wave tank. This is a numerical approximation of waves in a model basin, which allows the simulation of realistic sea states. The challenge here is to include complicated wave phenomena in the model.

A detailed discussion of various mathematical aspects of extreme wave modeling is given in [76], [120], [119]. In this thesis an important topic will be wave breaking, as happens in the near shore region, and is particularly important for tsunami studies. The difficulty here are the complex turbulent processes in the overturning wave, which will be approximated as a discontinuous flow. Another important topic in this thesis is wave focusing, which is important for rogue waves and stormy waves. Wave run-up and the interaction of waves with structures will not be considered here, but they will be part of future research.

The presence of a free surface and moving boundaries, such as wave makers, complicates the numerical modelling of wave phenomena. To cope with this we use specially designed variational finite element methods. Another important topic is mass and energy conservation, which are particularly important for long time simulations, as is frequently required in a numerical wave tank. We address these issues through the development of a (discrete) variational approach, which is directly associated with the conservation laws, in particular energy conservation. In the simulation of focusing waves it is also important to ensure numerical stability for large amplitude waves, which motivated us to use 
symplectic time integrators.

\subsection{Understanding water waves}

It is no surprise that the brightest minds were attracted by the secrets of water waves. The "Eureka" exclamation from Archimedes became a symbol of scientific excitement. An overview of the development of water wave theory can be found in [33], [36]. In this section we will give a summary of the main techniques used to study water waves.

\subsubsection{Experiments}

There are several ways to study water waves. The oldest way is of course observation. This is how Archimedes discovered his law and John Scott Russel discovered the soliton type of wave propagation. Field surveys and data collection of extreme events are very important for risk analysis, validation, and to provide realistic sea states in model experiments and computations [110], [137]. Sometimes data collection on a rare event, especially a historic one, reminds of a crime investigation with witness interviews and a detailed reconstruction of the event [114]. One can even try to look for traces more than 240 hundred years after the event, as did the authors of the prominent study [56] on the 1771 Meiwa Tsunami event.

As a variant of observation we have experiments. Experiments cover a wide range of wave phenomena. One can study wave focusing [25], [116], wave breaking [26], [80], [141], wave run up [19], [71], tsunami generation [43], [108], or wave-structure interaction, [28], [122]. A recent breakthrough was the observation of a freak wave in a laboratory, with a shape that corresponds to the analytic solution of the nonlinear Schrödinger equation - a so-called Peregrine breather [24].

There are many laboratories with various facilities all over the world. An overview on issues and specifications of laboratory experiments can be found in [37]. In these facilities prototype offshore structures and models of ships are tested in wave conditions mimicking a realistic sea state. Even though the concept is very natural, there are a number of difficulties. Not every condition can be modeled in a laboratory. For example, a floating oil platform in deep water is difficult to scale properly, due to the difference in the size of the platform, the risers and the depth of the ocean. Also, experiments require the construction of a model and the set-up of the experiment, which takes time, and needs to be accounted for in the design process of ships and offshore structures.

An example of a model basin is presented in Figure 1.5, which shows the wave basin of the Maritime Research Institute Netherlands (MARIN). In this advanced experimental facility it is possible to generate various wave forms by the movement of wave makers, and to study the wave impact on floating and fixed objects. A crucial question is then how one should move the wave maker in order to obtain the required wave phenomena. This is a non-trivial problem that has resulted in a number of studies, e.g. [1], [82], [89], 
[135], [152] and others. The special problem of generating focusing wave trains, which is important to study extreme wave events, is considered in [68], [70].

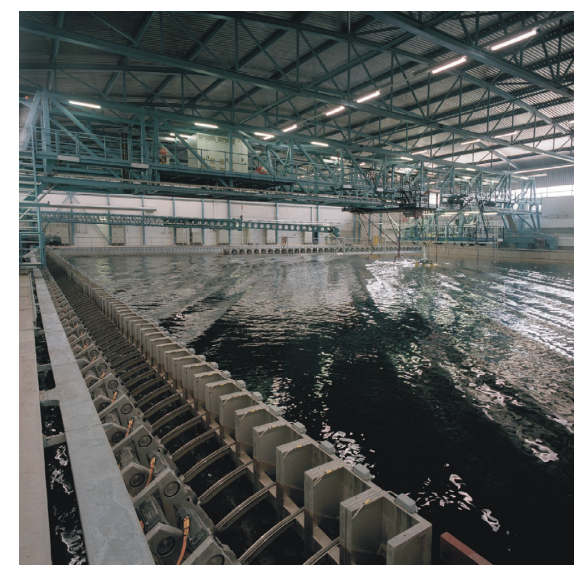

Figure 1.5: The MARIN facilities allow the creation of various wave types by moving wave makers at the side of the model basin.

\subsection{Modeling water waves}

\subsubsection{Mathematical wave models}

There are a number of mathematical models that describe water waves under different assumptions. A broad overview of wave models is given in the prominent books of Whitham [155] and Landau and Lifschitz [90]. The Navier-Stokes equations describe a general viscous incompressible fluid, with the Euler equations as inviscid limit. These equations describe general fluid flow, including a free surface, but for many types of free surface waves they are too complicated, therefore further simplifications are useful for practical applications. These simplifications can be, for example, the potential flow system, the shallow water equations, the Boussinesq equations, and many others.

Since there is a variety of wave models, one has to carefully consider the wave phenomena the model has to describe. We will discuss therefore the advantages and disadvantages of some widely used wave models. An important wave model is the shallow water equations, which are relatively simple and easy to solve numerically. This wave model allows discontinuous solutions - so called bores - as a simplification of complicated turbulent breaking waves. While mass and momentum are conserved across the discontinuity, energy is decreasing. This corresponds to the observations of breaking waves in [155] - where the water sprays and splashes while the wave is overturning, and, therefore, dissipates energy. 
As described in [121], when waves are breaking, energy is lost, but momentum is conserved. This results in a rise in horizontal shear or vertical vorticity, which becomes important when simulating breaking waves near the shore with two-dimensional models, such as the shallow water equations.

Among the downsides of the shallow water equations is the absence of dispersion (the property that waves with different wave length travel with different speed). In particular, waves are breaking when the nonlinearity outweights dispersion. Due to the absence of dispersion in the shallow water equations waves computed with this model tend to break too early in comparison with observations.

A second widely used wave model is the potential flow model. The potential flow equations are obtained from the Euler equations under the assumption that velocity field is irrotational (the velocity is the gradient of a scalar function called the potential), [73]. The potential flow model includes accurate dispersion, which makes the system attractive for wave focusing problems and the simulation of waves generated by a wave maker.

A unified wave model, embracing both the shallow water and potential flow system is useful when wave phenomena in various regions (e.g. open sea and near coastal region) need to be modeled simultaneously. Such a model was proposed by Cotter and Bokhove [30]. This model was obtained from an Eulerian variational principle with extended Clebsch variables [158], that only depend on the horizontal coordinates. By construction the velocity field consists of a three-dimensional potential flow part and a horizontally dependent vortical part. Under appropriate restrictions the new water wave model can be reduced to the shallow water equations, the potential flow model, and the variational Boussinesq model of Klopman et al., [82].

The Euler equations, which include three dimensional vorticity, is the next level of wave models. In Nurijanyan [112], a variational model for the rotating Euler equations is provided, and a linearised version of this model is applied to inertial waves.

A mathematical wave model can be solved either analytically or numerically. In practice, analytical methods can only be used to solve a wave model in special occasions. For potential flow a pseudo-analytical solution is provided in [47] and [55], for the shallow water equations solutions can be found in [4] and [140], and for the Euler equations [15] and [102]. There are also some known exact solutions for the Navier-Stokes equations [45] and [150], but practical wave phenomena might require a computation with numerical methods.

\subsubsection{Numerical methods}

Considering the difficulties in obtaining analytical solutions for the various wave models, numerical methods are of great practical importance. Since the power of computers is continuously growing, increasingly more sophisticated numerical models are becoming available. Still, there is a pay-off between the complexity of the wave model and its practical use. In particular, one has to define the necessary phenomena that need to be included 
in the simulation model and to consider the required computational power. Imagine there is an underwater earthquake with the danger of a tsunami. It is then important to compute the possible wave shapes generated by the motion of the ocean bottom, to simulate their propagation and to define as soon as possible areas under threat. In this case, the simplest model, the shallow water model, will do the job. On the other hand, when one works with focusing waves or wave-current interactions, where dispersion or three dimensional vorticity play a role, more sophisticated wave models need to be used.

Provided the main interest of this research, we give now a brief overview of numerical methods suitable for the simulation of water waves in a model basin.

\subsubsection{Numerical wave tank}

Apart from studying wave phenomena in the ocean or near coastal region, wave models are also important to study wave generation in a model basin or even to replace experiments in a model basin all together. This motivates the development of a numerical wave tank (NWT), which simulates waves in a laboratory (or in the ocean). In 1976 Longuet-Higgins and Cokelet simulated an overturning wave using potential flow theory with a mixed Eulerian-Lagrangian method in combination with a boundary integral equation formulation. Almost simultaneously, in 1977, Faltinsen [46] implemented a similar method for a floating body in water waves. One of the first to employ a finite element method for the nonlinear potential flow equation were Wu, Wang and Eatock Taylor [41] in 1994. A spectral method using a Dirichlet to Neumann operator was introduced by Craig and Sulem in 1993, [32]. For a review of the numerical methods for nonlinear water waves before 2000, see [77] and [142].

For complicated breaking waves the meshless Smoothed Particle Hydrodynamics (SPH) methods are an interesting new development and currently under active study, [67 and [143]. Even though SPH is a convenient method to simulate wave-structure interaction or extreme waves, the method suffers from substantial artificial dissipation, which does not make it suitable for accurate long time simulations or wave propagation studies.

One of the first three-dimensional numerical wave tanks was developed by Grilli et al [58]. It contains an arbitrary bottom topography, sloping beach and the possibility to include wave makers. The nonlinear potential flow equation is solved with a combination of a boundary element method (BEM) and a mixed Eulerian-Lagrangian technique to compute the free surface motion. This NWT was used both for the study of breaking waves [63], and freak waves [51]. Another three dimensional NWT based on BEM is presented in [127], [128].

An alternative to the widely used boundary element methods for nonlinear water waves is provided by finite element methods, which currently are under active development [101], [145], [151]. These methods have a number of advantages. In particular, they allow to deal with complicated moving boundaries. There are also other benefits for finite element methods: the numerical discretization results in sparse matrices suitable for fast solvers, they have the potential to be extended to more complicated flow models, 
such as Euler and the Navier-Stokes equations, which is not possible with BEM. Discontinuous finite element methods, which result in a local, element based discretization with only a weak coupling to neighboring elements are particularly useful for wave problems requiring mesh adaptation using local mesh refinement and parallel computations [138]. For problems with moving boundaries, such as free surface waves, it is beneficial to use a space-time approach, which has attracted much attention recently, [23], [75]. This approach considers time as an extra dimension, making a time-dependent $n$-dimensional problem an $n+1$ dimensional steady state problem. In a space-time framework it is easier to satisfy the geometric conservation law on time-dependent domains, where the interior mesh movement is induced by the free surface and boundary motion, see Lesoinne and Farhat, [94]. Space-time DG finite element methods for linear free surface wave problems were developed in Ambati [3], and for nonlinear flows in Van der Vegt and Xu [144]. Understanding time as an extra dimension to the spatial problem also leads to the construction of novel time stepping methods using time-discontinuous elements. For an extensive overview of numerical wave tanks, see [40].

In order to facilitate the validation of numerical simulation models for water waves MARIN organized in 2010 a special workshop [22], for which it conducted a number of experiments in its facilities. These experiments included wave focusing over a flat bottom and irregular waves over an uneven bottom. These experiments provided a wealth of data, which was used in [60], [91] for a model validation and will also be extensively used in this study.

\subsubsection{Variational dynamics}

Many water wave problems can be described by a variational principle, which provides a description of the state of a physical system as the extremum of a functional. Originally this idea was explored in problems of finding the shortest path between two points or the least time of light is travelling between two points (the principle of least action). Euler was first to establish a connection between the principle of least action and a tendency of physical bodies to occupy a state with minimal total potential energy. Lagrange and, later, Hamilton reformulated classical mechanics using an energy-related functional, now referred to as Lagrangian. For an extensive overview of mathematical methods of classical mechanics, see [6].

In a variational principle, the complete system is expressed as a single functional a Lagrangian - which is the difference between potential and kinetic energy. Within a variational framework it is relatively easy to introduce variable transformations, establish conservation laws [10] and stability theorems, and it is also a good starting point for a numerical discretization. The representation of the dynamics using a variational principle is concise and elegant.

The variational formulation also provides a straightforward way to obtain simplified systems that preserve the symmetry of the original, more complicated system. An example related to wave phenomena is the simplification of the velocity potential in Miles' 
formulation as is done by Klopman [82]. A wide range of consistent approximations is discussed in [31].

Hamiltonian mechanics is a reformulation of Lagrangian mechanics to investigate the symmetry properties of a system. There is a direct connection between the variational principle and the symmetry properties of Hamiltonian systems and conservation laws as is established by Noether's theorem [111].

The first developments in variational fluid dynamics were made by Clebsch in 1859 [29], Hargreaves in 1908 [66], and Bateman in 1944 [8]. They considered the pressure as a Lagrangian density, but did not include the boundary conditions at the free surface.

Inspired by Whitham [153], [154], Luke [98], introduced a variational principle for free surface waves in a bounded basin. He also sketched the possibility to add vorticity to the velocity field. Independently, Zakharov [159], Broer [20], and Miles [106], proved the Hamiltonian structure of the nonlinear potential flow equations. The pair of canonically conjugate variables for potential flow free surface waves are $\eta$ and $\phi_{S}$ - the free surface elevation and the velocity potential at the free surface, which act as generalized coordinate and momentum. Variational Boussinesq type approximations were developed by Broer [20], Miles and Salmon [107], Milder [105], and Klopman [82]. For a review, see Finlayson [48] and Grimshaw [59].

The variational formulation allows a straightforward numerical discretization. Indeed, one just needs to approximate the variables with e.g. a spatial finite element or spectral representation. The discrete variational principle will then inherit the conservation of energy and phase space of the continuous variational formulation. One has to be, however, careful with the time integration, otherwise the variational structure will not be preserved and the numerical discretization might suffer from numerical instabilities. For this purpose symplectic time integrators for Hamiltonian systems were developed [64]. These time integrators preserve conservation laws and ensure that the discrete solution has no energy drift and its fluctuations are bounded if the original system is energy conservative.

Recent developments in the field of (discrete) Hamiltonian fluid dynamics can be found in [17], [27], [78], [82].

\subsection{Outline}

The outline of this thesis is as follows. In Chapter 2 we consider a new variational water wave model, developed by Cotter and Bokhove [30]. This model inherits the benefits of the shallow water model and the potential flow model. It has a three-dimensional velocity field consisting of the full three-dimensional potential velocity field plus extra, horizontally dependent, components. This construction implies that only the vertical vorticity component is nonzerd 1 . The model includes dispersion and allows breaking waves. In this thesis the Hamiltonian structure of the model is shown and the Poisson bracket to

\footnotetext{
${ }^{1}$ It is pointed out to us by T. J. Bridges in 2012, that an absence of the horizontal components of vorticity might lead to an instability, likewise it happens for Benney's shallow water equations [18].
} 
describe the evolution of the system is obtained, see also [54]. The model reductions to a Green-Naghdi type of system and to the Boussinesq model of Klopman [82] are demonstrated under certain assumptions. Also, a variational approach to derive jump conditions to represent bores is presented. The approach is based on the work of Wakelin [148], where the domain is split into two parts by a bore and jump conditions at the bore are obtained. When the wave model is reduced to the shallow water limit the jump relations at the bore reduce to the well-known shallow water jump relations [90], [155]. Also, a variational analog of the Reynolds transport theorem is proved.

In Chapter 3 we use a variational approach to derive novel time integration methods. The time integration method is based on a discontinuous Galerkin finite element discretization in time, with a specially derived numerical flux. With this approach we were able to derive the well known symplectic Euler, symplectic modified midpoint and Störmer-Verlet time integration methods. Also, new time integrators were obtained. For all novel variational time integrators the order of accuracy, linear stability conditions, symplecticity, and dispersion of the schemes are analyzed. The original motivation for this research was to obtain stable time integration methods for forced systems, and the newly developed techniques are applied to forced pendulums and, later in Chapter 4 for waves generated by a wave maker. Also, damped systems are considered.

In Chapter 4 we consider waves generated by a wave maker in a numerical wave tank [52]. The model we use is the nonlinear potential flow equation. The numerical method is a mixed space continuous - time discontinuous Galerkin method with linear basis functions, providing second order of accuracy in space and time. A comparison with laboratory experiments provided by the Maritime Research Institute Netherlands (MARIN) is made and found to be satisfactory. Also in Chapter 4 we combine the novel third order time integrator developed in Chapter 3 and the described variational numerical method to compute nonlinear water waves. In this autonomous test case the numerical method shows promising results.

Finally, conclusions and recommendation for future work will be discussed in Chap$\operatorname{ter} 5$ 


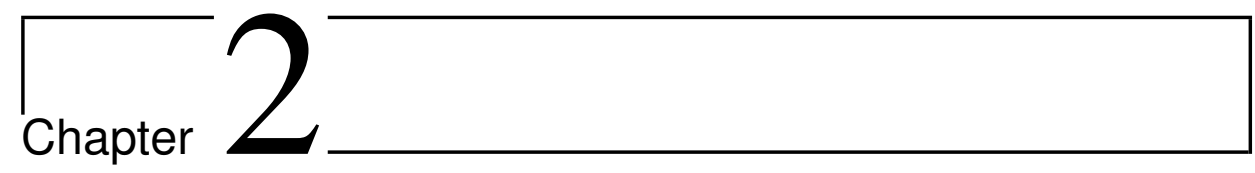

\section{Horizontal circulation and jumps in Hamiltonian wave models}

We are interested in the modeling of wave-current interactions around surf zones at beaches. Any model that aims to predict the onset of wave breaking at the breaker line needs to capture both the nonlinearity of the wave and its dispersion. We have therefore formulated the Hamiltonian dynamics of a new water wave model, incorporating both the shallow water and pure potential flow water wave models as limiting systems. It is based on a Hamiltonian reformulation of the variational principle derived by Cotter and Bokhove [30] by using more convenient variables. Our new model has a threedimensional velocity field consisting of the full three-dimensional potential velocity field plus extra horizontal velocity components. This implies that only the vertical vorticity component is nonzero. Variational Boussinesq models and Green-Naghdi equations, and extensions thereof, follow directly from the new Hamiltonian formulation after using simplifications of the vertical flow profile. Since the full water wave dispersion is retained in the new model, waves can break. We therefore explore a variational approach to derive jump conditions for the new model and its Boussinesq simplifications.

The content of this chapter is presented as a journal article [54] written in collaboration with J.J.W. van der Vegt and O. Bokhove.

\subsection{Introduction}

The beach surf zone is defined as the region of wave breaking and white capping between the moving shore line and the (generally time-dependent) breaker line. Let us consider wave propagation from deeper water to shallow water regions. The start of the surf zone on the offshore side is at the breaker line where sustained wave breaking 
begins. It demarcates the points where the nonlinearity of the waves becomes strong enough to outweigh dispersion. The waves thus start to overturn. From the point of breaking till the shore, the waves lose energy and generate vorticity. A mathematical model that can predict the onset of wave breaking at the breaker line will need to capture both the nonlinearity of the waves and their dispersion. Moreover the model has to include vorticity effects to simulate wave-current interactions.

Various mathematical models are used to describe water waves. A popular model for smooth waves in deep water is the potential flow model, but its velocity field does not include vorticity. In the near-shore region, vorticity effects are, however, important. When obliquely incident waves shoal in shallow water, steepen and break, a horizontal shear or vertical vorticity is generated. On semi-enclosed or enclosed beaches, this leads to an overall circulation induced by wave breaking. A classical hydraulic model for the surf zone is the shallow water model. The complicated, turbulent three-dimensional wave breaking is approximated in this model by discontinuities or so-called bores. These are special relations holding across the jumps connecting the right and left states of the flow. Mass and momentum are conserved across the discontinuity, while energy is not, as can be expected from observing the white capping zone of fine-scale splashes and sprays in the broken wave [154]. Shallow water waves are not dispersive, and these waves tend to break too early in comparison with real, dispersive waves. Boussinesq models include internal wave dispersion to a higher degree of accuracy, but dispersion always seems to beat nonlinearity. Therefore wave overturning tends to be prevented in these models. The variational Boussinesq model proposed by Klopman [82] could be a notable exception, but it is based on the Ansatz of potential flow. In three dimensions, a purely potential-flow model cannot be extended by inclusion of bores and hydraulic jumps as a simple model to represent wave breaking. The reason is that at least some vorticity has to be generated by bores that have non-uniformities along their jump line as was shown in [124], [121], and [123].

We therefore seek to develop a more advanced model that includes both the shallow water approximation of breaking waves as bores and the accurate dispersion of the potential flow model. Such a model was obtained by Cotter and Bokhove [30] from a parent Eulerian variational principle with extended Clebsch variables, in which the vortical parts only depended on the horizontal coordinates. This restricts the vorticity to have a vertical component only. Extended Clebsch variables may, however, be less convenient algebraically and also yield a larger phase space of variables. We therefore reformulate this system in terms of surface velocity, velocity potential and water depth, and derive the Hamiltonian structure including its Poisson bracket. This new water wave model can be reduced to the shallow water equations, the potential flow model, and the Boussinesq model of Klopman [82] under corresponding restrictions. The Green-Naghdi equations emerge from the variational Boussinesq model by introducing a parabolic potential flow profile in the Poisson bracket, as well as another, columnar approximation of the velocity in the Hamiltonian.

Finally, a new variational approach to derive jump conditions across bores is also ex- 
plored. It is inspired by the work of Waklein [148] for stationary shock or jump conditions for the shallow water equations. These results have been extended to moving shocks in shallow water based on the variational principles for the relevant Clebsch variables. Naturally, this approach allows us to obtain jump conditions for the new water wave model as well. The jump relations can be implemented in any system with a variational and Hamiltonian structure, but not every system with a Hamiltonian structure allows shocks or discontinuities to persist in time. For example, it was shown in [42] that the Green-Naghdi system has an unsteady undular bore, i.e., an initial discontinuity in the free surface and velocity expands instantly into smooth undulations. It is therefore necessary to analyze the energy loss across jumps, and juxtapose this analysis between the original and our new extended Green-Naghdi system.

The outline of the chapter is as follows. First, a systematic derivation of the new Hamiltonian formulation will be given starting from a slightly adapted formulation of the variational principle from [30] in $\$ 2.2$ Subsequently, we show in $\$ 2.3$ how it can be reduced to limiting systems, such as the shallow water equations, the potential flow model, the Boussinesq model of Klopman [82] and an extended version of the GreenNaghdi equations. In 2.5, a variational approach to derive jump conditions is given, starting from the well-known Rankine-Hugoniot or jump conditions for the shallow water equations. We end with conclusions in 2.6

\subsection{New water wave model}

\subsubsection{Variational principle}

Consider an incompressible fluid at time $t$ in a three-dimensional domain bounded by solid surfaces and a free surface, with horizontal coordinates $x, y$, and vertical coordinate $z$. The water depth is denoted by $h=h(x, y, t)$. There exists a parent Eulerian variational principle for incompressible flow with a free surface. Its three-dimensional velocity field $\vec{U}=\vec{U}(x, y, z, t)=(u, v, w)^{T}$, with $(\cdot)^{T}$ denoting the transpose, contains both potential and rotational parts and is represented as

$$
\vec{U}=\nabla \phi+\vec{\pi}_{j} \nabla \vec{l}_{j}
$$

through extended Clebsch variables: the velocity potential $\phi=\phi(x, y, z, t)$, the threedimensional fluid parcel label $\vec{l}=\vec{l}(x, y, z, t)$ and the corresponding Lagrange multiplier vector $\vec{\pi}=\vec{\pi}(x, y, z, t), j=1,2,3$. Such a representation describes a velocity field containing all three components of vorticity $\nabla \times \vec{U}$. In order to avoid confusion, indices are also introduced in (2.1) and the Einstein convention for repeated indices is used, such that $\vec{\pi}_{j} \nabla \vec{l}_{j}=\pi_{1} \nabla l_{1}+\pi_{2} \nabla l_{2}+\pi_{3} \nabla l_{3}$. This velocity representation is similar to the expression (3.9) in [130]. Also Lin [96] used two three-dimensional vector Clebsch variables to introduce a vorticity for superfluids. As in [158], we see, that a pair of extended Clebsch vectors suffices for the generalized form to be complete. 
When only the potential velocity field $\nabla \phi(x, y, z, t)$ is used, there is no vorticity. In contrast, a shallow water velocity field includes the vertical component of vorticity. Similarly, in an Eulerian variational principle with planar Clebsch variables that only depend on the horizontal coordinates the vertical component of vorticity is retained. This component is constant throughout the whole water column and flows with helicity [88] are thus excluded by construction.

Cotter and Bokhove [30] derived novel water wave dynamics from the parent Eulerian variational principle which includes two limits: Luke's variational principle giving the classical potential water wave model and a principle for depth-averaged shallow water flows based on planar Clebsch variables. At least conceptually, the novel variational principle follows readily from the parent principle with two-dimensional label and multiplier fields $\vec{l}$ and $\vec{\pi}$ depending only on the two-dimensional horizontal coordinates and time. Hence, they no longer depend on the vertical coordinate $z$. In his prominent paper, Luke [98] has mentioned about the possibility of the introduction of Clebsch potentials into the variational principle for the rotational case. In contrast, we do not use Clebsch scalar variables, but extended vector Clebsch variables.

Extended Clebsch variables are, however, not convenient to work with. We therefore reduce the model to a more compact and conventional form. This reduction from six variables $\{\phi, h, \vec{l}, \vec{\pi}\}$ to four more conventional variables $\left\{\phi, h, \vec{u}^{*}\right\}$ is undertaken in a Hamiltonian setting. The latter variables involve a new velocity $\vec{u}^{*}$, which is a suitable horizontal velocity.

The variational principle of [30] has the following form:

$$
0=\delta \int_{0}^{T} \mathcal{L}[\vec{l}, \vec{\pi}, \phi, h] \mathrm{d} t=\delta \int_{0}^{T} \int_{\Omega_{H}} \int_{b}^{b+h} \partial_{t} \phi+\vec{\pi} \cdot \partial_{t} \vec{l} \mathrm{~d} z \mathrm{~d} x \mathrm{~d} y+\mathcal{H} \mathrm{d} t,
$$

where the horizontal part of the domain is $\Omega_{H}$; the single-valued free surface boundary lies at $z=h(x, y, t)+b(x, y)$, with $h(x, y, t)$ the water depth and $b(x, y)$ a given, fixed topography; and, $t$ is time, its derivative is $\partial_{t}$, and $T$ a final time. The variational derivative is defined as

$$
\delta \int_{0}^{T} \mathcal{L}[\vec{l}, \vec{\pi}, \phi, h] \mathrm{d} t=\lim _{\epsilon \rightarrow 0} \int_{0}^{T} \frac{\mathcal{L}[\vec{l}+\epsilon \delta \vec{l}, \vec{\pi}+\epsilon \delta \vec{\pi}, \phi+\epsilon \delta \phi, h+\epsilon \delta h]-\mathcal{L}[\vec{l}, \vec{\pi}, \phi, h]}{\epsilon} \mathrm{d} t .
$$

The component of the velocity with vortical parts is contained in

$$
\overrightarrow{\mathrm{v}}(x, y, t)=\pi_{j}(x, y, t) \nabla l_{j}(x, y, t), \quad \text { with } \quad j=1,2
$$

and three-dimensional gradient $\nabla$. Thus, the entire three-dimensional velocity field is represented by

$$
\vec{U}(x, y, z, t)=\nabla \phi(x, y, z, t)+\vec{v}(x, y, t)
$$


combining the potential velocity $\nabla \phi(x, y, z, t)$ and the planar velocity $\vec{v}(x, y, t)$. The relevant Hamiltonian, the sum of kinetic and potential energies, equals

$$
\mathcal{H}=\mathcal{H}[\vec{l}, \vec{\pi}, \phi, h]=\int_{\Omega_{H}} \int_{b}^{b+h} \frac{1}{2}|\nabla \phi+\vec{v}|^{2} \mathrm{~d} z+\frac{1}{2} g\left((h+b)^{2}-b^{2}\right)-g h H_{0} \mathrm{~d} x \mathrm{~d} y,
$$

with $g$ the acceleration of the Earth's gravity, and $H_{0}$ a still water reference level. This Hamiltonian contains the available potential energy, due to the additional subtraction of the rest level contribution, cf. [136].

As is shown in [30], the variational formulation of the new system is similar to Hamiltonian classical mechanics, and becomes

$$
\begin{aligned}
& \delta \phi: \quad \nabla^{2} \phi+\nabla \cdot \vec{v}=0, \\
& \delta h: \quad \partial_{t} \phi_{s}=-\frac{\delta \mathcal{H}}{\delta h}, \quad \delta \phi_{s}: \quad \partial_{t} h=\frac{\delta \mathcal{H}}{\delta \phi_{s}}, \\
& \delta(h \vec{\pi}): \quad \partial_{t} \vec{l}=-\frac{\delta \mathcal{H}}{\delta(h \vec{\pi})}, \quad \delta \vec{l}: \quad \partial_{t}(h \vec{\pi})=\frac{\delta \mathcal{H}}{\delta \vec{l}},
\end{aligned}
$$

with the free surface velocity potential

$$
\phi_{s}=\phi_{s}(x, y, t)=\phi(x, y, z=h+b, t) .
$$

Hamiltonian variations are equal to

$$
\begin{aligned}
\frac{\delta \mathcal{H}}{\delta h}= & \frac{1}{2}\left|\nabla_{H} \phi_{s}+\vec{v}\right|^{2}+g\left(h+b-H_{0}\right)-\vec{v} \cdot \overrightarrow{\vec{u}} \\
& -\frac{1}{2}\left(\partial_{z} \phi\right)_{s}^{2}\left(1+\left|\nabla_{H}(h+b)\right|^{2}\right), \\
\frac{\delta \mathcal{H}}{\delta \phi_{s}}= & \left(\partial_{z} \phi\right)_{s}\left(1+\left|\nabla_{H}(h+b)\right|^{2}\right)-\left(\nabla_{H} \phi_{s}+\vec{v}\right) \cdot \nabla_{H}(h+b), \\
\frac{\delta \mathcal{H}}{\delta\left(h \vec{\pi}_{i}\right)}= & \overline{\vec{u}} \cdot \nabla \vec{l}_{i}, \\
\frac{\delta \mathcal{H}}{\delta \vec{l}_{i}}= & -\nabla \cdot\left(h \overline{\vec{u}}_{i}\right) .
\end{aligned}
$$

In the above expressions, we used the depth-averaged horizontal velocity

$$
\overline{\vec{u}}(x, y, t)=\frac{1}{h} \int_{b}^{b+h} \vec{U}_{H} \mathrm{~d} z
$$

where $\vec{U}_{H}=(u, v)^{T}$ is the horizontal component of the velocity $\vec{U}$, and $\nabla_{H}=\left(\partial_{x}, \partial_{y}\right)^{T}$ is the horizontal component of the gradient. Here the subscript $(.)_{s}$ denotes variables at 
the free surface. To obtain these results, we also employed the relation

$$
\delta\left(\phi_{s}\right)=(\delta \phi)_{s}+\left(\partial_{z} \phi\right)_{s} \delta h
$$

and a similar one for $\partial_{t}\left(\phi_{s}\right)$.

The pairs $(\vec{l}, h \vec{\pi})$ and $\left(\phi_{s}, h\right)$ at the free surface are canonically conjugated. Thus the Hamiltonian dynamics arising from (2.2)-(2.7) is canonical and takes the form

$$
\frac{d \mathcal{F}}{d t}=\{\mathcal{F}, \mathcal{H}\}=\int_{\Omega_{H}} \frac{\delta \mathcal{F}}{\delta h} \frac{\delta \mathcal{H}}{\delta \phi_{s}}-\frac{\delta \mathcal{F}}{\delta \phi_{s}} \frac{\delta \mathcal{H}}{\delta h}+\frac{\delta \mathcal{F}}{\delta(h \vec{\pi})} \cdot \frac{\delta \mathcal{H}}{\delta \vec{l}}-\frac{\delta \mathcal{F}}{\delta \vec{l}} \cdot \frac{\delta \mathcal{H}}{\delta(h \vec{\pi})} \mathrm{d} x \mathrm{~d} y
$$

Subsequent substitution for $\mathcal{F}$ of one of these variables $\vec{l}, h \vec{\pi}, \phi_{s}$ or $h$-rewritten as a functional in 2.12 - in turn yields 2.7). Than following [109] we take $h\left(\vec{x}^{\prime}, t\right)=$ $\int_{\Omega_{H}} h(\vec{x}, t) D\left(\vec{x}-\vec{x}^{\prime}\right) \mathrm{d} x \mathrm{~d} y$, etc, where $D\left(\vec{x}-\vec{x}^{\prime}\right)$ is Dirac delta-function.

\subsubsection{Reduction of Hamiltonian dynamics}

The aim is to reduce the number of variables in the Hamiltonian formulation from the set $\left\{\phi, \phi_{s}, h, \vec{l}, h \vec{\pi}\right\}$ to the set $\left\{\varphi \equiv \phi-\phi_{s}, h, \vec{u}^{*}\right\}$. Doing so removes the reference to the label fields and their conjugates and yields a reduction by two fields. This transformation is achieved via variational techniques. The surface velocity $\vec{u}^{*}$ is now split into a potential and rotational part, which allows us to reformulate the Hamiltonian dynamics. The key observaton is that the velocity field $(2.5)$ can be rewritten as

$$
\vec{U}=\vec{u}^{*}+\nabla\left(\phi-\phi_{s}\right) \equiv \vec{u}^{*}+\nabla \varphi
$$

by introducing a surface velocity

$$
\vec{u}^{*}(x, y, t)=\nabla \phi_{s}(x, y, t)+\vec{v}(x, y, t) .
$$

Upon using this in (2.6), the resulting Hamiltonian becomes

$$
\mathcal{H}\left[\phi, \phi_{s}, h, \vec{u}^{*}\right]=\int_{\Omega_{H}} \int_{b}^{b+h} \frac{1}{2}\left|\vec{u}^{*}+\nabla\left(\phi-\phi_{s}\right)\right|^{2} \mathrm{~d} z+\frac{1}{2} g\left((h+b)^{2}-b^{2}\right)-g h H_{0} \mathrm{~d} x \mathrm{~d} y .
$$

Consequently, instead of the seven fields used in 2.6, we can use five fields. The question is whether a similar reduction can be achieved from the Poisson bracket, thus closing the Hamiltonian formulation in the new variables. The subsequent derivation has a technical character and readers can safely jump to the next subsection, in which the result is stated. 
We relate the two sets of variational derivatives by taking variations of a functional $\mathcal{F}$ in terms of the prognostic variables

$$
\begin{aligned}
\delta \mathcal{F} & =\int_{\Omega_{H}} \frac{\delta \mathcal{F}}{\delta \phi_{s}} \delta \phi_{s}+\frac{\delta \mathcal{F}}{\delta h} \delta h+\frac{\delta \mathcal{F}}{\delta(h \vec{\pi})} \cdot \delta(h \vec{\pi})+\frac{\delta \mathcal{F}}{\delta \vec{l}} \cdot \delta \vec{l} \mathrm{~d} x \mathrm{~d} y \\
& =\int_{\Omega_{H}} \frac{\delta \mathcal{F}}{\delta\left(h \vec{u}^{*}\right)} \cdot \delta\left(h \vec{u}^{*}\right)+\frac{\delta \mathcal{F}}{\delta h} \delta h \mathrm{~d} x \mathrm{~d} y,
\end{aligned}
$$

which connects variations with respect to the different sets of variables. After using (2.14) with 2.4 in the above, an integration by parts and using Gauss' law, we obtain

$$
\begin{aligned}
\frac{\delta \mathcal{F}}{\delta \phi_{s}} & =-\nabla \cdot\left(h \frac{\delta \mathcal{F}}{\delta\left(h \vec{u}^{*}\right)}\right), \\
\frac{\delta \mathcal{F}}{\delta \vec{l}_{j}} & =-\nabla \cdot\left(h \vec{\pi}_{j} \frac{\delta \mathcal{F}}{\delta\left(h \vec{u}^{*}\right)}\right), \\
\left.\frac{\delta \mathcal{F}}{\delta h}\right|_{\phi_{s}} & =\frac{\delta \mathcal{F}}{\delta\left(h \vec{u}^{*}\right)} \cdot \nabla \phi_{s}+\left.\frac{\delta \mathcal{F}}{\delta h}\right|_{h u^{*}}, \\
\frac{\delta \mathcal{F}}{\delta\left(h \vec{\pi}_{j}\right)} & =\frac{\delta \mathcal{F}}{\delta\left(h \vec{u}^{*}\right)} \cdot \nabla \vec{l}_{j},
\end{aligned}
$$

where the index notation is $i, j, k=1,2$. Boundary contributions in the above calculation vanish because at solid vertical boundaries $\hat{\vec{n}}_{H} \cdot \delta \mathcal{F} / \delta\left(h \vec{u}^{*}\right)=0$ with the horizontal outward normal $\hat{\vec{n}}_{H}$, or because $h=0$ at the water line. Substitution of 2.17) into 2.12) yields the transformed Hamiltonian formulation in momentum variables

$$
\begin{aligned}
& \frac{d \mathcal{F}}{d t}=\int_{\Omega_{H}} \frac{\delta \mathcal{H}}{\delta h} \nabla_{i}\left(h \frac{\delta \mathcal{F}}{\delta\left(h \vec{u}_{i}^{*}\right)}\right)-\frac{\delta \mathcal{F}}{\delta h} \nabla_{i}\left(h \frac{\delta \mathcal{H}}{\delta\left(h \vec{u}_{i}^{*}\right)}\right) \\
& +h \vec{u}_{k}^{*}\left(\frac{\delta \mathcal{H}}{\delta\left(h \vec{u}_{k}^{*}\right)} \nabla_{i} \frac{\delta \mathcal{F}}{\delta\left(h \vec{u}_{i}^{*}\right)}-\frac{\delta \mathcal{F}}{\delta\left(h \vec{u}_{k}^{*}\right)} \nabla_{i} \frac{\delta \mathcal{H}}{\delta\left(h \vec{u}_{i}^{*}\right)}\right) \\
& +\frac{\delta \mathcal{F}}{\delta\left(h \vec{u}_{i}^{*}\right)} \frac{\delta \mathcal{H}}{\delta\left(h \vec{u}_{k}^{*}\right)}\left(\nabla_{i}\left(h \vec{u}_{k}^{*}\right)-\nabla_{k}\left(h \vec{u}_{i}^{*}\right)\right) \mathrm{d} x \mathrm{~d} y,
\end{aligned}
$$

where we employed the chain rule, notation $\nabla_{1}=\partial_{x}, \nabla_{2}=\partial_{y}$ for clarity's sake, the relation

$$
\nabla_{i} h \nabla_{k} \phi_{s}-\nabla_{k} h \nabla_{i} \phi_{s}=\nabla_{i}\left(h \nabla_{k} \phi_{s}\right)-\nabla_{k}\left(h \nabla_{i} \phi_{s}\right)
$$

and $\nabla_{i} \nabla_{k} \phi_{s}=\nabla_{k} \nabla_{i} \phi_{s}$.

\subsubsection{Hamiltonian dynamics of new water wave model}

We complete the derivation by stating the Hamiltonian dynamics of the new water wave model. In the next two sections, two limiting systems and Boussinesq approximations will be based directly on this new Hamiltonian formulation. The final step is to 
transform the Hamiltonian formulation 2.18 with respect to the set $\left\{h, h \vec{u}^{*}\right\}$ into one with respect to $\left\{h, \vec{u}^{*}\right\}$, using the relations

$$
\frac{\delta \mathcal{F}}{\delta\left(h \vec{u}^{*}\right)}=\frac{1}{h} \frac{\delta \mathcal{F}}{\delta \vec{u}^{*}} \quad \text { and }\left.\quad \frac{\delta \mathcal{F}}{\delta h}\right|_{h u^{*}}=\left.\frac{\delta \mathcal{F}}{\delta h}\right|_{u^{*}}-\frac{\vec{u}^{*}}{h} \frac{\delta \mathcal{F}}{\delta \vec{u}^{*}} .
$$

By substitution of 2.20) into 2.18, we obtain the desired Hamiltonian formulation in the new variables

$$
\frac{d \mathcal{F}}{d t}=\int_{\Omega_{H}}-q \frac{\delta \mathcal{F}}{\delta \vec{u}^{*}} \cdot \frac{\delta \mathcal{H}}{\delta \vec{u}^{*}}-\frac{\delta \mathcal{F}}{\delta h} \nabla \cdot \frac{\delta \mathcal{H}}{\delta \vec{u}^{*}}+\frac{\delta \mathcal{H}}{\delta h} \nabla \cdot \frac{\delta \mathcal{F}}{\delta \vec{u}^{*}} \mathrm{~d} x \mathrm{~d} y,
$$

with $(\cdot)^{\perp}$ the rotated vector as in $\left(\vec{u}^{*}\right)^{\perp} \equiv\left(-u_{2}^{*}, u_{1}^{*}\right)^{T}$, and note that the gradients $\nabla$ are effectively two dimensional as they operate on functions independent of $z$. The potential vorticity is defined as

$$
\begin{aligned}
q \equiv\left(\partial_{x} v-\partial_{y} u\right) / h & =\left(\partial_{x} \mathrm{v}_{2}-\partial_{y} \mathrm{v}_{1}\right) / h \\
& =\left(\partial_{x} u_{2}^{*}-\partial_{y} u_{1}^{*}\right) / h .
\end{aligned}
$$

No integration by parts was required in the previous transformation. Since only the difference of variables $\phi$ and $\phi_{s}$ appears, we introduce a modified potential $\varphi=\phi-\phi_{s}$, zero at the free surface. Hence, we can slightly simplify 2.15 to

$$
\mathcal{H}\left[\varphi, h, \vec{u}^{*}\right]=\int_{\Omega_{H}} \int_{b}^{b+h} \frac{1}{2}\left|\vec{u}^{*}+\nabla \varphi\right|^{2} \mathrm{~d} z+\frac{1}{2} g\left((h+b)^{2}-b^{2}\right)-g h H_{0} \mathrm{~d} x \mathrm{~d} y .
$$

Specification of $\mathcal{F}$ in 2.21, in turn, and use of 2.23, yields the equations of motion

$$
\begin{aligned}
\partial_{t} h & =-\nabla \cdot \frac{\delta \mathcal{H}}{\delta \vec{u}^{*}}, \\
\partial_{t} \vec{u}^{*} & =-\nabla \frac{\delta \mathcal{H}}{\delta h}-q \frac{\delta \mathcal{H}}{\delta \vec{u}^{*}}
\end{aligned}
$$

using Hamiltonian variations

$$
\begin{aligned}
\delta h: & \frac{\delta \mathcal{H}}{\delta h}=B, \\
\delta \vec{u}^{*}: & \frac{\delta \mathcal{H}}{\delta \vec{u}^{*}}=h \overline{\vec{u}}, \\
\delta \varphi: & -\frac{\delta \mathcal{H}}{\delta \varphi}=\nabla \cdot \vec{u}^{*}+\nabla^{2} \varphi=0,
\end{aligned}
$$

with the depth-weighted horizontal velocity vector in 2.10 redefined as

$$
\overline{\vec{u}}(x, y, t)=\frac{1}{h} \int_{b}^{b+h}\left(\vec{u}^{*}+\nabla_{H} \varphi\right) \mathrm{d} z
$$


and the Bernoulli function

$$
B=\frac{1}{2}\left|\vec{u}^{*}\right|^{2}+g\left(h+b-H_{0}\right)-\frac{1}{2}\left(\partial_{z} \varphi\right)_{s}^{2}\left(1+\left|\nabla_{H}(h+b)\right|^{2}\right) .
$$

Note that $\delta \mathcal{H} / \delta \varphi=0$ acts here as a constraint, since it does not play a role in the prognostics.

The final system of equations in the new free surface variables equals

$$
\begin{aligned}
\partial_{t} h+\nabla \cdot(h \overline{\vec{u}}) & =0, \\
\partial_{t} \vec{u}^{*}+\nabla B+q(h \overline{\vec{u}})^{\perp} & =0,
\end{aligned}
$$

with the elliptic equation for $\varphi$ in the interior

$$
\nabla^{2} \varphi=-\nabla \cdot \vec{u}^{*}
$$

The boundary conditions for $\varphi$ in 2.29$)$ are $\vec{n} \cdot\left(\vec{u}^{*}+\nabla \varphi\right)=0$ at solid walls, with $\vec{n}$ the exterior normal vector, and $\varphi=0$ at the free surface.

We can also formulate the new system in a (conservative) form, which will become relevant for the derivation of jump conditions later. Using definitions 2.26 and 2.13, the key step is to notice that

$$
\begin{aligned}
\partial_{t}(h \overline{\vec{u}}) & =\partial_{t}\left(\int_{b}^{b+h} \vec{U}_{H} \mathrm{~d} z\right) \\
& =\left(u_{s}, v_{s}\right)^{T} \partial_{t} h+h \partial_{t} \vec{u}^{*}+\int_{b}^{b+h} \nabla_{H} \partial_{t} \varphi \mathrm{d} z .
\end{aligned}
$$

The term $h \partial_{t} \vec{u}^{*}$ can now be obtained from 2.28b. The integral term is rewritten by interchanging the order of integral and horizontal gradient, thus introducing surface and bottom boundary contributions. The next step is to rewrite the continuity equation $2.25 \mathrm{c}$, or $\nabla \cdot \vec{U}=0$, by integrating over depth, to obtain

$$
\partial_{t} h=w_{s}-u_{s} \partial_{x}(b+h)-v_{s} \partial_{y}(b+h),
$$

in which we use the full velocity evaluated at the free surface and we note that $w_{s}=$ $\left(\partial_{z} \varphi\right)_{s}$. Hence, we can evaluate each term in 2.30 further. Substitution of 2.31) into 2.30 leads to terms like $-u_{s}^{2} \partial_{x}(b+h)-u_{s} v_{s} \partial_{y}(b+h)$, which can be rewritten in terms of depth-integrated fluxes of the three-dimensional velocity. For example, $u_{s}^{2} \partial_{x}(b+h)$ can be determined from

$$
\partial_{x} \int_{b}^{b+h} u^{2} \mathrm{~d} z=\int_{b}^{b+h} 2 u \partial_{x} u \mathrm{~d} z+u_{s}^{2} \partial_{x}(b+h)-u_{b}^{2} \partial_{x} b
$$


in which subscript $(\cdot)_{b}$ in $u_{b}$ denotes that horizontal velocity $u$ is evaluated at the bottom $z=b$. For gradients at the free surface, we extensively use relations like

$$
(\boldsymbol{\nabla} \varphi)_{s}=\left(\boldsymbol{\nabla} \varphi_{s}\right)-\left(\partial_{z} \phi\right)_{s} \boldsymbol{\nabla}(h+b)=-w_{s} \boldsymbol{\nabla}(h+b),
$$

since by definition $\varphi_{s}=0$. In addition, we use the condition that the velocity normal to the bottom boundary is zero.

Without going through further details, the reformulated equations of motion resulting after some calculations become as follows:

$$
\partial_{t}\left(\begin{array}{c}
h \\
h \bar{u} \\
h \bar{v}
\end{array}\right)+\nabla \cdot\left(\begin{array}{c}
\mathcal{F}_{0} \\
\mathcal{F}_{1} \\
\mathcal{F}_{2}
\end{array}\right)=\left(\begin{array}{c}
0 \\
S_{1} \\
S_{2}
\end{array}\right),
$$

with the flux tensor

$$
\left(\begin{array}{c}
\mathcal{F}_{0} \\
\mathcal{F}_{1} \\
\mathcal{F}_{2}
\end{array}\right)=\left(\begin{array}{cc}
h \bar{u} & h \bar{v} \\
A & \int_{b}^{h+b} u v \mathrm{~d} z \\
\int_{b}^{h+b} u v \mathrm{~d} z & C
\end{array}\right),
$$

where $\overline{\vec{u}}=(\bar{u}, \bar{v})^{T}$,

$$
\begin{aligned}
A= & \int_{b}^{h+b}\left(u^{2}-\frac{|\vec{U}|^{2}}{2}+\frac{\left|\vec{u}^{*}\right|^{2}}{2}-\partial_{t} \varphi\right) \mathrm{d} z+\frac{g h^{2}}{2} \\
& -\frac{h}{2} w_{s}^{2}\left(1+|\nabla(h+b)|^{2}\right), \\
C= & \int_{b}^{h+b}\left(v^{2}-\frac{|\vec{U}|^{2}}{2}+\frac{\left|\vec{u}^{*}\right|^{2}}{2}-\partial_{t} \varphi\right) \mathrm{d} z+\frac{g h^{2}}{2} \\
& -\frac{h}{2} w_{s}^{2}\left(1+|\nabla(h+b)|^{2}\right),
\end{aligned}
$$

and

$$
\begin{aligned}
\left(S_{1}, S_{2}\right)^{T}= & \left(-g h+\frac{1}{2} w_{s}^{2}\left(1+|\nabla(h+b)|^{2}\right)\right. \\
& \left.+\left(\partial_{t} \varphi+\frac{1}{2}|\vec{U}|^{2}-\frac{1}{2}\left|\vec{u}^{*}\right|^{2}\right)_{b}\right)\left(\partial_{x} b, \partial_{y} b\right)^{T}
\end{aligned}
$$

\subsection{Shallow water and potential flow limits}

The shallow water and potential flow models emerge as limiting systems of the new water wave model, as will be shown next. The new water wave model reduces to the 
potential flow equations when we take $\vec{U}=\nabla \phi$ in the Hamiltonian (2.6) and only use the terms with $h$ and $\phi_{s}$ in the Poisson bracket 2.12). The Hamiltonian of the system then takes the form

$$
\mathcal{H}=\mathcal{H}[\phi, h]=\int_{\Omega_{H}} \int_{b}^{b+h} \frac{1}{2}|\nabla \phi|^{2} \mathrm{~d} z+\frac{1}{2} g\left((h+b)^{2}-b^{2}\right)-g h H_{0} \mathrm{~d} x \mathrm{~d} y .
$$

The shallow water limit is obtained when we restrict $\phi=\phi(x, y, z, t)$ to be the surface potential $\phi_{s}=\phi_{s}(x, y, t)$ in the extended Luke's variational principle (2.2) such that $\varphi=$ 0 . The velocity field then reduces to $\vec{U}(x, y, t)=\vec{u}^{*}(x, y, t)=\nabla \phi_{s}(x, y, t)+\vec{v}(x, y, t)$. This change yields $\overline{\vec{u}}=\vec{u}^{*}(x, y, t)$, and the Hamiltonian dynamics remains 2.21) but with the Hamiltonian

$$
\mathcal{H}=\mathcal{H}[\overline{\vec{u}}, h]=\int_{\Omega_{H}} \frac{1}{2} h|\overline{\vec{u}}|^{2}+\frac{1}{2} g\left((h+b)^{2}-b^{2}\right)-g h H_{0} \mathrm{~d} x \mathrm{~d} y,
$$

cf. [130]. In this case the second equation in (2.28) is transformed to the depth-averaged shallow water momentum equation

$$
\partial_{t} \overline{\vec{u}}+\nabla B+q h \overline{\vec{u}}^{\perp}=0,
$$

with $q h=\partial_{x} \bar{v}-\partial_{y} \bar{u}$ and $B=(1 / 2)|\overline{\vec{u}}|^{2}+g(h+b)$.

\subsection{Hamiltonian Boussinesq reductions of new model}

The idea to approximate the vertical structure of the flow velocity beneath the free surface was first applied by Boussinesq [16] for the description of fairly long surface waves in shallow water. Such Boussinesq-type water wave models are widely used in coastal and maritime enginering. Alternatively, these models can be viewed as a Galerkin or Ritz discretization of the velocity potential in the vertical coordinate $z$ only. When such an expansion of the velocity potential in terms of vertical profiles is substituted directly into the variational principle, a so-called variational Boussinesq model results. It depends on only the horizontal coordinates and time. An example is the variational Boussinesq model of Klopman [82]. These authors also sketched how to add a vorticity term to the potential flow model, but in an ad hoc fashion. In contrast, we apply the Galerkin or Ritz method directly to the Hamiltonian formulation of our new water wave model, and thus systematically maintain the vertical component of the vorticity. A Boussinesq-type wave model can subsequently also be discretized in the horizontal directions and time. It is unclear whether such a secondary discretization instead of one directly applied to the original model in three dimensions is more advantageous, or not. The advantage of first discretizing the vertical direction may be that these reduced Boussinesq models are more amenable to mathematical analysis. The analysis of jump conditions, explored later, perhaps illustrates this point. 


\subsubsection{Variational Boussinesq model}

In the Ritz method, the velocity potential is approximated as a linear combination of $M$ basis functions, such that

$$
\varphi(x, y, z, t)=\sum_{m=1}^{M} f_{m}\left(z ; h, b, k_{m}\right) \psi_{m}(x, y, t),
$$

with shape functions $f_{m}$ and variables $\psi_{m}(x, y, t)$. By definition, the shape functions are chosen such that $f_{m}=0$ at the free surface $z=h+b$ in a strong sense. The functions $k_{m}(x, y)$ may be used as optional shape parameters, but we assume them to be known and fixed a priori. Note that due to the direct substitution of 2.40) into 2.23, the Hamiltonian remains by default positive. The expansion 2.40 implies that the condition $\delta \mathcal{H} / \delta \varphi=0$ is replaced by

$$
\frac{\delta \mathcal{H}}{\delta \psi_{m}}=0, \quad m=1, . ., M .
$$

The simplest model of practical interest has one shape function $(M=1)$ :

$$
\varphi(x, y, z, t)=f(z ; b, h) \psi(x, y, t)
$$

and the following expression for the flow velocity is obtained

$$
\begin{aligned}
\nabla_{H} \varphi & =f \nabla_{H} \psi+\left(\partial_{b} f\right) \psi \nabla_{H} b+\left(\partial_{h} f\right) \psi \nabla_{H} h, \\
\partial_{z} \phi & =\left(\partial_{z} f\right) \psi .
\end{aligned}
$$

In principle it seems that a substitution of 2.43 into the Hamiltonian 2.23 combined with the Hamiltonian dynamics 2.21) suffices to define a reduced Boussinesq model. The challenge, however, is to satisfy the bottom boundary condition:

$$
w=\psi \partial_{z} f=\left(\vec{u}^{*}+\nabla_{H}(f \psi)\right) \cdot \nabla_{H} b \quad \text { at } \quad z=b
$$

in a strong sense. Satisfaction of this bottom boundary condition in a weak sense, as in numerical approaches, appears to be less well explored (in Boussinesq water wave models).

It is therefore common (cf. [82]) to assume the bed slopes to be mild, such that $\nabla_{H} b \approx 0$ and 2.43 can be approximated as

$$
\begin{aligned}
\nabla_{H} \varphi & =f \nabla_{H} \psi+\left(\partial_{h} f\right) \psi \nabla_{H} h, \\
\partial_{z} \phi & =\left(\partial_{z} f\right) \psi .
\end{aligned}
$$

Consequently, 2.44 reduces to $\partial_{z} f=0$, which is more easily imposed on the vertical profile $f(z ; b, h)$ in a strong sense. After introducing 2.45) into the Hamiltonian 2.23), 
the result is

$$
\begin{aligned}
\mathcal{H}= & \int_{\Omega_{H}} \int_{b}^{h+b} \frac{1}{2}\left|\vec{u}^{*}+f \nabla_{H} \psi+\left(\partial_{h} f\right) \psi \nabla_{H} h\right|^{2} \\
& \quad+\frac{1}{2}\left(\psi \partial_{z} f\right)^{2} \mathrm{~d} z+\frac{1}{2} g\left((h+b)^{2}-b^{2}\right) \mathrm{d} x \mathrm{~d} y \\
= & \int_{\Omega_{H}} \frac{1}{2} h\left|\vec{u}^{*}\right|^{2}+\frac{1}{2} F|\nabla \psi|^{2}+P \nabla \psi \cdot \vec{u}^{*} \\
& +\frac{1}{2} \psi^{2}\left(K+G|\nabla h|^{2}\right)+Q \psi \vec{u}^{*} \cdot \nabla h+R \psi \nabla \psi \cdot \nabla h \\
& +\frac{1}{2} g\left((h+b)^{2}-b^{2}\right) \mathrm{d} x \mathrm{~d} y,
\end{aligned}
$$

where $F, K, G, P, Q, R$ are functions of $h$, provided in Appendix 2.7.1. Variations of 2.46 with respect to $h, \vec{u}^{*}$ remain as in 2.25a and 2.25b, but the elliptic equation $2.25 \mathrm{c}$, here resulting from the variation of $\psi$, is reduced to

$$
\begin{gathered}
\delta \psi: \quad\left(K+G|\nabla h|^{2}\right) \psi+Q \vec{u}^{*} \cdot \nabla h+R \nabla \psi \cdot \nabla h \\
-\nabla \cdot\left(F \nabla \psi+P \vec{u}^{*}+R \psi \nabla h\right)=0 .
\end{gathered}
$$

Perhaps, it is a matter of taste whether (2.47) is simpler than (2.29). The reduction in dimensionality, however, is clear, as 2.29) is an elliptic equation in a three-dimensional domain, while 2.47) holds in the corresponding horizontal domain defined by the (singlevalued) free surface. These variations, combined with Hamiltonian dynamics 2.21, again yield the system (2.24). The expressions for the depth-averaged horizontal velocity and the Bernoulli function are, however, modified as follows

$$
\begin{aligned}
h \overline{\vec{u}} & =\int_{b}^{b+h}\left(\vec{u}^{*}+f \nabla_{H} \psi+\left(\partial_{h} f\right) \psi \nabla_{H} h\right) \mathrm{d} z \\
& =h \vec{u}^{*}+P \nabla \psi+Q \psi \nabla h, \\
B & =\frac{1}{2}\left|\vec{u}^{*}\right|^{2}+g(h+b)+\mathcal{R}^{*},
\end{aligned}
$$

with $\mathcal{R}^{*}$ defined as

$$
\begin{aligned}
\mathcal{R}^{*}= & \frac{1}{2} F^{\prime}|\nabla \psi|^{2}+\frac{1}{2}\left(K^{\prime}+G^{\prime}|\nabla h|^{2}\right) \psi^{2}+ \\
& \left(P^{\prime} \boldsymbol{\nabla} \psi+Q^{\prime} \psi \boldsymbol{\nabla} h\right) \cdot \vec{u}^{*}+R^{\prime} \psi \boldsymbol{\nabla} \psi \cdot \nabla h- \\
& \boldsymbol{\nabla} \cdot\left(G \psi^{2} \boldsymbol{\nabla} h+Q \psi \vec{u}^{*}+R \psi \boldsymbol{\nabla} \psi\right),
\end{aligned}
$$

and primed variables denote $P^{\prime}=\mathrm{d} P / \mathrm{d} h$, etc. In the variations of 2.46 with respect to $\psi$ and $h$, boundary contributions cancel either because the velocity normal to vertical 
walls is zero or because $h=0$ at the water line. Note that the approximated system of equations again takes the form 2.28) augmented with the elliptic equation (2.47) for $\psi$.

When, for example, we consider a parabolic vertical profile

$$
f=f^{(p)}=\frac{1}{2} \frac{(z-b)^{2}-h^{2}}{h},
$$

then the Hamiltonian becomes

$$
\begin{aligned}
\mathcal{H}= & \int_{\Omega_{H}} \frac{1}{2} h\left|\vec{u}^{*}-\frac{2}{3} \psi \nabla h-\frac{1}{3} h \boldsymbol{\nabla} \psi\right|^{2}+\frac{1}{2} g\left((h+b)^{2}-b^{2}\right) \\
& +\frac{1}{90} h|\psi \nabla h-h \boldsymbol{\nabla} \psi|^{2}+\frac{1}{6} h \psi^{2} \mathrm{~d} x \mathrm{~d} y,
\end{aligned}
$$

which is positive-definite, since the water depth $h>0$. The integrals $F, K, G, P, Q, R$ are readily calculated explicitly, see Appendix 2.7.1. Consequently, one finds that the relevant expressions become

$$
\begin{aligned}
h \overline{\vec{u}}= & h \vec{u}^{*}-\frac{1}{3} h^{2} \nabla \psi-\frac{2}{3} h \psi \nabla h \\
B= & \frac{1}{2}\left|\vec{u}^{*}\right|^{2}+g(h+b)+\mathcal{R}^{*}, \\
\mathcal{R}^{*}= & \frac{1}{5} h^{2}|\nabla \psi|^{2}+\frac{1}{6}\left(1+\frac{7}{5}|\nabla h|^{2}\right) \psi^{2} \\
& \quad-\frac{2}{3}(h \boldsymbol{\nabla} \psi+\psi \nabla h) \cdot \vec{u}^{*}+\frac{2}{5} h \psi \nabla \cdot \psi \nabla h \\
& \quad-\nabla \cdot\left(\frac{7}{15} h \psi^{2} \nabla h-\frac{2}{3} h \psi \vec{u}^{*}+\frac{1}{5} h^{2} \psi \nabla \psi\right), \\
& h \psi\left(\frac{1}{3}+\frac{7}{15}|\nabla h|^{2}\right)-\left(\frac{2}{3} h \vec{u}^{*}-\frac{1}{5} h^{2} \nabla \psi\right) \cdot \nabla h- \\
& \nabla \cdot\left(\frac{2}{15} h^{3} \nabla \psi-\frac{1}{3} h^{2} \vec{u}^{*}+\frac{1}{5} h^{2} \psi \nabla h\right)=0 .
\end{aligned}
$$

In summary, we derived and extended the variational Boussinesq model within a Hamiltonian framework, by a Ritz and mild-slope approximation of the vertical potential flow profile, while systematically including the vertical component of the vorticity. The difference between our model and Klopman's model is in the velocity field, which in our case includes the vertical vorticity. The surface velocity representation $\vec{u}^{*}(x, y, t)=\nabla \phi_{s}(x, y, t)+\vec{v}(x, y, t)$ namely replaces the representation used by Klopman $\vec{u}^{*}(x, y, t)=\nabla \phi_{s}(x, y, t)$ from the onset.

\subsubsection{Green-Naghdi limit}

The Green-Naghdi equations are obtained from a variational principle under the assumption that the fluid moves in vertical columns, as was shown by [107]. The model 
is sufficiently dispersive that shocks cannot be maintained as an initial discontinuity disperses into smooth undulations instantly, as was shown in [42]. We will show that the Green-Naghdi equations can be derived from the variational Boussinesq model with a parabolic potential flow profile via an additional approximation to the Hamiltonian.

Instead of (2.51), the shape function is taken to be $\left(h^{2}-(z-b)^{2}\right) / 2$. Hence, the modified velocity potential becomes

$$
\varphi(x, y, z, t)=\frac{h^{2}-(z-b)^{2}}{2} \psi(x, y, t) .
$$

Of course, this is equivalent to 2.42) with 2.51), i.e., $\varphi^{(p)}=\left((z-b)^{2}-h^{2}\right) \psi^{(p)} /(2 h)$, provided we redefine $\psi^{(p)}=-h \psi$. With the mild-slope approximation, the velocity field then becomes

$$
\begin{aligned}
\vec{u}_{H} & =\vec{u}^{*}+\frac{1}{2} \nabla_{H}\left(\left(h^{2}-z^{2}\right) \psi\right), \\
w & =\varphi_{z}=-z \psi .
\end{aligned}
$$

The expressions 2.52 and 2.53 are now immediately valid given this substitution of $\psi^{(p)}$ in terms of $h$ and $\psi$. The depth-averaged velocity thus follows from 2.53a as

$$
\overline{\vec{u}}=\frac{1}{h} \int_{b}^{h+b} \vec{u} \mathrm{~d} z=\vec{u}^{*}+h \psi \nabla h+\frac{h^{2}}{3} \nabla \psi .
$$

Likewise, the Hamiltonian 2.52 becomes

$$
\mathcal{H}\left[h, \vec{u}^{*}, \psi\right]=\int_{\Omega} \frac{1}{2} h|\overrightarrow{\vec{u}}|^{2}+\frac{1}{6} h^{3} \psi^{2}+\frac{1}{2} g\left((h+b)^{2}-b^{2}\right)+\beta \frac{h^{5}|\nabla \psi|^{2}}{90} \mathrm{~d} x \mathrm{~d} y,
$$

where we added a "switch" parameter $\beta=\{0,1\}$ to be used later, and rephrased the formulation in terms of $\overline{\vec{u}}$. Note, however, that $\overline{\vec{u}}$ is defined in terms of $h, \psi$ and $\vec{u}^{*}$ in 2.56.

The Hamiltonian dynamics 2.24) combined with variations of (2.57) with respect to $h$ and $\vec{u}^{*}$ (using 2.56) again lead to the dynamics 2.28). Either via 2.47) or more directly by taking variations with respect to $\psi$ for fixed $h$ and $\vec{u}^{*}$ in (2.57), one obtains

$$
\psi=\nabla \cdot \overline{\vec{u}}+\frac{\beta}{15 h^{3}} \nabla \cdot\left(h^{5} \nabla \psi\right) .
$$

This is an elliptic equation for $\psi$ once one uses 2.56 to reexpress $\overline{\vec{u}}$. The Bernoulli function follows either by rearranging 2.49) or from the variation of 2.57 with respect to $h$, and takes the form

$$
\begin{aligned}
B=\frac{1}{2}|\overline{\vec{u}}|^{2} & +\frac{1}{2} h^{2} \psi^{2}+g(h+b)-\frac{1}{3} h^{2} \overline{\vec{u}} \cdot \nabla \psi \\
& -h^{2} \psi \nabla \cdot \overline{\vec{u}}-h \psi \overline{\vec{u}} \cdot \nabla h+\beta h^{2} \frac{h^{2}|\nabla \psi|^{2}}{18} .
\end{aligned}
$$


The Green-Naghdi system arises by keeping the relation 2.56 between $\overline{\vec{u}}$ and $\vec{u}^{*}$ and the Hamiltonian dynamics (2.24), but simplifying the Hamiltonian (2.57) to one with $\beta=0$. Hence, the variations with respect to $h$ and $\psi$ and the equations (2.58) simplify to

$$
\begin{aligned}
& \psi=\boldsymbol{\nabla} \cdot \overline{\vec{u}}, \\
& \begin{aligned}
B= & \frac{1}{2}|\overline{\vec{u}}|^{2}-\frac{1}{2} h^{2}(\nabla \cdot \overline{\vec{u}})^{2}+g(h+b)-\frac{1}{3} h^{2} \overline{\vec{u}} \cdot \nabla(\nabla \cdot \overline{\vec{u}}) \\
& \quad-(h \boldsymbol{\nabla} \cdot \overline{\vec{u}})(\overline{\vec{u}} \cdot \nabla h) .
\end{aligned}
\end{aligned}
$$

This simplification of the Hamiltonian is equivalent to the substitution of yet another three-dimensional velocity

$$
\vec{u}=\tilde{\vec{u}}=(\overline{\vec{u}},-z \psi)^{T}
$$

into the original Hamiltonian 2.23. Consequently, 2.59a is a continuity equation given a columnar horizontal velocity $\vec{u}$ and that $w=-z \psi$. Due to this approximation, the velocity field given by 2.60 has non-zero horizontal vorticity components:

$$
\vec{\omega}=\nabla \times(\bar{u}, \bar{v}, w)^{T}=\left(\partial_{y} w,-\partial_{x} w, \partial_{x} \bar{v}-\partial_{y} \bar{u}\right),
$$

in contrast to the original system with $\beta=1$.

The explicit expression $\psi=\nabla \cdot \overline{\vec{u}}$ in 2.59a allows us to reformulate the system to the standard Green-Naghdi model, as follows

$$
\begin{aligned}
& \partial_{t} h+\nabla \cdot(h \overline{\vec{u}})=0, \\
& \partial_{t} \overline{\vec{u}}+(\overline{\vec{u}} \cdot \nabla) \overline{\vec{u}}+g \nabla(h+b)= \\
& \quad h \boldsymbol{\nabla} h\left(\nabla \cdot \partial_{t} \overline{\vec{u}}+(\overline{\vec{u}} \cdot \nabla)(\nabla \cdot \overline{\vec{u}})-(\nabla \cdot \overline{\vec{u}})^{2}\right)+ \\
& \quad \frac{h^{2}}{3} \nabla\left(\nabla \cdot \partial_{t} \overline{\vec{u}}+(\overline{\vec{u}} \cdot \nabla)(\nabla \cdot \overline{\vec{u}})-(\nabla \cdot \overline{\vec{u}})^{2}\right),
\end{aligned}
$$

cf. equation (1) in [14]. In summary, we have recovered the original Green-Naghdi system from a reformulation and approximation of the variational Boussinesq model. This approximation is Hamilonian, but consists of using another, columnar approximation of the three-dimensional velocity in the Hamiltonian rather than employing the parabolic potential profile that is still used in the Poisson bracket.

\subsection{Jump conditions for bores}

The most widely used model to describe wave propagation and breaking near the shore - the shallow water equations - doesn't contain dispersion. Nevetheless, dispersive effects during wave propagation in coastal zones can be important. We illustrate the subtle interplay between dispersion and dissipation with the bore-soliton-splash experiment [13]. This experiment is conducted in a wave channel with a sluice at the begining and a constriction at the end. The sluice gate locks in a higher water level than in the main part 
of the channel. At some point this gate is opened instantly and a soliton is formed (see Fig. 2.1, which breaks quickly because its amplitude is too high and propagates further as a hydraulic jump or bore (see Fig. 2.2. During its propagation the bore loses energy and amplitude, such that just before the constriction, it turns into the smooth soliton again (see Fig. 2.3. The first reflected soliton draws a through at the contraction in which the lower second soliton crashes and splashes up (see Fig. 2.4). We mention that there were three "nearly" similar reruns of the experiment, and we used the best images from any of these three [160]. The discussion concerns runs 3, 6, and 8 (performed at the opening of the education plaza at the University of Twente in 2010). The propagation of a smooth, broken and rejuvenated soliton is an illustration of the balance and imbalance between nonlinearity and dispersion. Therefore, a theoretical and numerical model to describe such a phenomena has to include dispersion and has to deal with breaking waves, in which nonlinearity dominates.

Following ideas of Wakelin [148], we further develop a technique to derive jump conditions from variational principles. To illustrate the intricate details of this approach, the well-known jump conditions for bores are derived first for the depth-averaged shallow water equations in one dimension. Subsequently, the jump conditions for the new water wave model in two horizontal dimensions are obtained and its limitation to the well-known 2D shallow water jump conditions is shown. The jump conditions for the closely related variational Boussinesq and the Green-Naghdi models are especially interesting as far as it is known that the Green-Naghdi model cannot maintain discontinuities since dispersion is too strong [42]. The situation for the variational Boussinesq model is unknown, while we know that the full water wave model with its potential flow water waves can lead to overturning and breaking waves.

\subsubsection{D Jump conditions for shallow water equations}

Consider a bore propagating in a channel $\Omega$. The domain $\Omega$ is split into two parts: $\Omega_{1}$ lying behind the bore and $\Omega_{2}$ lying in front of the bore, as shown in Fig. 2.5 Between these domains there is a moving boundary $\partial \Omega_{b}$ corresponding to the instant bore position at $x=x_{b}(t)$. The key point is to consider the two domains separately and couple them at $x_{b}$. If we consider one subdomain, then the moving bore interface is akin to a piston wave maker. It will be shown that variational techniques are a natural way to obtain the bore relations. The coupling establishes that there is an energy loss at the interior bore boundary.

Let us assume that the domain $\Omega$ has solid wall boundaries and a flat bottom. The state to the left from the interior bore boundary $x_{b}(t)$ is given by the depth $h^{-}$and horizontal velocity $u^{-}$, and the one to the right by $h^{+}$and $u^{+}$. The bore speed $S=\dot{x}_{b} \equiv \mathrm{d} x_{b} / \mathrm{d} t$. The shallow water velocity potential considered at the free surface is $\phi \equiv \phi_{s}(x, t)$, with corresponding depth-averaged horizontal velocity $u=\phi_{x} \equiv \partial_{x} \phi$. The analog of the extended Luke's variational principle 2.2 -2.6 for the depth-averaged shallow water 
system is

$$
\begin{aligned}
& 0=\delta \int_{0}^{T} \mathcal{L}[\phi, h, t] \mathrm{d} t=\delta \int_{0}^{T}\left(\int_{0}^{x_{b}^{-}}\left(-h \phi_{t}-\frac{1}{2} h\left(\phi_{x}\right)^{2}-\frac{1}{2} g h^{2}+g h H_{0}\right) \mathrm{d} x\right. \\
& \left.+\int_{x_{b}^{+}}^{L}\left(-h \phi_{t}-\frac{1}{2} h\left(\phi_{x}\right)^{2}-\frac{1}{2} g h^{2}+g h H_{0}\right) \mathrm{d} x\right) \mathrm{d} t,
\end{aligned}
$$

in which we used the more compact notation $\phi_{t} \equiv \partial_{t} \phi$, etc. and $x_{b}^{-}=\lim _{\epsilon \rightarrow 0^{-}}\left(x_{b}+\epsilon\right)$ and $x_{b}^{+}=\lim _{\epsilon \rightarrow 0^{+}}\left(x_{b}+\epsilon\right)$.

Taking variations of 2.63 we get

$$
\begin{aligned}
& 0=\delta \int_{0}^{T} \mathcal{L}[\phi, h, t] \mathrm{d} t= \\
& \int_{0}^{T}\left(\int_{0}^{x_{b}^{-}}+\int_{x_{b}^{+}}^{L}\right)\left(\left(-\phi_{t}-\frac{1}{2}\left(\phi_{x}\right)^{2}-g h+g H_{0}\right) \delta h-h \delta \phi_{t}-h \phi_{x} \delta \phi_{x}\right) \mathrm{d} x \\
& +\left(-h \partial_{t} \phi-\frac{1}{2} h\left(\phi_{x}\right)^{2}-\frac{1}{2} g h^{2}+g h H_{0}\right)^{-} \delta x_{b} \\
& \left.-\left(-h \partial_{t} \phi-\frac{1}{2} h\left(\phi_{x}\right)^{2}-\frac{1}{2} g h^{2}+g h H_{0}\right)^{+} \delta x_{b}\right) \mathrm{d} t .
\end{aligned}
$$

When we work out some terms in detail, we obtain

$$
\begin{aligned}
& \int_{0}^{T}\left(\int_{0}^{x_{b}^{-}}+\int_{x_{b}^{+}}^{L}\right) h \delta \phi_{t} \mathrm{~d} x \mathrm{~d} t=\int_{0}^{T}\left(\left(\int_{0}^{x_{b}^{-}}+\int_{x_{b}^{+}}^{L}\right)\left((h \delta \phi)_{t}-h_{t} \delta \phi\right) \mathrm{d} x\right) \mathrm{d} t \\
& =\int_{0}^{T}\left(-\left(\int_{0}^{x_{b}^{-}}+\int_{x_{b}^{+}}^{L}\right) h_{t} \delta \phi \mathrm{d} x+\frac{d}{d t}\left(\left(\int_{0}^{x_{b}^{-}}+\int_{x_{b}^{+}}^{L}\right) h \delta \phi \mathrm{d} x\right)-\dot{x}_{b} h^{-}(\delta \phi)^{-}+\dot{x}_{b} h^{+}(\delta \phi)^{+}\right) \mathrm{d} t,
\end{aligned}
$$


and

$$
\begin{aligned}
& \int_{0}^{T}\left(\left(\int_{0}^{x_{b}^{-}}+\int_{x_{b}^{+}}^{L}\right) h \phi_{x} \delta \phi_{x} \mathrm{~d} x\right) \mathrm{d} t=\int_{0}^{T}\left(\left(\int_{0}^{x_{b}^{-}}+\int_{x_{b}^{+}}^{L}\right)\left(\left(h \phi_{x} \delta \phi\right)_{x}-\left(h \phi_{x}\right)_{x} \delta \phi\right) \mathrm{d} x\right) \mathrm{d} t \\
& =\int_{0}^{T}\left(-\left(\int_{0}^{x_{b}^{-}}+\int_{x_{b}^{+}}^{L}\right)\left(h \phi_{x}\right)_{x} \delta \phi \mathrm{d} x+\left(h \phi_{x} \delta \phi\right)^{-}-\left(h \phi_{x} \delta \phi\right)^{+}\right) \mathrm{d} t .
\end{aligned}
$$

Using the endpoint conditions $(\delta \phi)_{t=0}=(\delta \phi)_{t=T},(\delta h)_{t=0}=(\delta h)_{t=T}$, the resulting variations become

$$
\begin{aligned}
& 0=\delta \int_{0}^{T} \mathcal{L}[\phi, h, t] \mathrm{d} t= \\
& \int_{0}^{T}\left(\left(\int_{0}^{x_{b}^{-}}+\int_{x_{b}^{+}}^{L}\right)\left(\left(-\phi_{t}-\frac{1}{2}\left(\phi_{x}\right)^{2}-g h+g H_{0}\right) \delta h+\left(h_{t}+\left(h \phi_{x}\right)_{x}\right) \delta \phi\right) \mathrm{d} x\right. \\
& +\left[h \phi_{t}+\frac{1}{2} h\left(\phi_{x}\right)^{2}+\frac{1}{2} g h^{2}-g h H_{0}\right] \delta x_{b} \\
& \left.+S h^{-}(\delta \phi)^{-}-S h^{+}(\delta \phi)^{+}-\left(h \phi_{x} \delta \phi\right)^{-}+\left(h \phi_{x} \delta \phi\right)^{+}\right) \mathrm{d} t,
\end{aligned}
$$

where we defined the jump $[f] \equiv f^{+}-f^{-}$for an aribitrary quantity $f$. Under the assumption that the velocity field can at most contain discontinuities, it follows that the velocity potential at the interface $\phi_{b}=\phi\left(x_{b}\right)=\phi\left(x_{b}^{-}\right)=\phi\left(x_{b}^{+}\right)$must be continuous. For variations over the interface variables we use the relation $\delta\left(\phi_{b}\right)=(\delta \phi)_{b}+\left(\phi_{x}\right)_{b} \delta x_{b}$, and then obtain

$$
\begin{aligned}
& 0=\delta \int_{0}^{T} \mathcal{L}[\phi, h, t] \mathrm{d} t= \\
& \int_{0}^{T}\left(\left(\int_{0}^{x_{b}^{-}}+\int_{x_{b}^{+}}^{L}\right)\left(\left(-\phi_{t}-\frac{1}{2}\left(\phi_{x}\right)^{2}-g h+g H_{0}\right) \delta h+\left(h_{t}+\left(h \phi_{x}\right)_{x}\right) \delta \phi\right) \mathrm{d} x\right. \\
& \left.+\left[h \phi_{t}+\frac{1}{2} h\left(\phi_{x}\right)^{2}+\frac{1}{2} g h^{2}-g h H_{0}\right] \delta x_{b}-\left[S h-h \phi_{x}\right] \delta \phi_{b}+\left[S h \phi_{x}-h \phi_{x}^{2}\right] \delta x_{b}\right) \mathrm{d} t .
\end{aligned}
$$

The final step is to use the equation for $\phi_{t}$ following from the arbitrary variation $\delta h$ to combine the terms with $\delta x_{b}$. 
Subsequently, variations with respect to $\phi, h, x_{b}, \phi_{b}$ produce the following system of equations

$$
\begin{aligned}
\delta \phi: & \partial_{t} h+\left(h \phi_{x}\right)_{x}=0, \text { at }\left[0, x_{b}^{-}\right) \cup\left(x_{b}^{+}, L\right], \\
\delta h: & \partial_{t} \phi+\frac{1}{2}\left(\phi_{x}\right)^{2}+g\left(h-H_{0}\right)=0, \text { at }\left[0, x_{b}^{-}\right) \cup\left(x_{b}^{+}, L\right], \\
\delta \phi_{b}: & {\left[h\left(S-\phi_{x}\right)\right]=0, \quad \text { at } x=x_{b}, } \\
\delta x_{b}: & {\left[h \phi_{x}^{2}-h S \phi_{x}+\frac{1}{2} g h^{2}\right]=0, \quad \text { at } x=x_{b} . }
\end{aligned}
$$

Equations 2.69a - 2.69b are the well-known shallow water equatons. Using $u=\phi_{x}$ they can be represented as

$$
\partial_{t} h+(h u)_{x}=0 \quad \text { and } \quad \partial_{t} u+u u_{x}+g h_{x}=0,
$$

in $\left[0, x_{b}^{-}\right) \cup\left(x_{b}^{+}, L\right]$, and the jump conditions 2.69c) and 2.69d) are reformulated as

$$
\begin{array}{ll}
\delta \phi_{b}: & {[h(S-u)]=0,} \\
\delta x_{b}: & {\left[h(S-u)^{2}+\frac{1}{2} g h^{2}\right]=0 .}
\end{array}
$$

These are the well-known Rankine-Hugoniot conditions for a moving bore in the shallow water equations.

An important property of hydraulic jumps in shallow water is the loss of energy similar to the rise of entropy for shocks in compressible fluid dynamics. It corresponds to the observation that breaking waves spray into many droplets losing mechanical energy in the turbulent processes. The energy of the system is given by the Hamiltonian. Taking the time derivative of the Hamiltonian and using (2.70), we obtain

$$
\begin{aligned}
\frac{\mathrm{d} \mathcal{H}}{\mathrm{d} t} & =\frac{\mathrm{d}}{\mathrm{d} t}\left(\int_{0}^{x_{b}^{-}}+\int_{x_{b}^{+}}^{L}\right)\left(\frac{1}{2} h u^{2}+\frac{1}{2} g h^{2}\right) \mathrm{d} x \\
& =\left(\int_{0}^{x_{b}^{-}}+\int_{x_{b}^{+}}^{L}\right)\left(\frac{1}{2} h u^{2}+\frac{1}{2} g h^{2}\right)_{t} \mathrm{~d} x-\dot{x}_{b}\left[\frac{1}{2} h u^{2}+\frac{1}{2} g h^{2}\right] \\
& =\left(\int_{0}^{x_{b}^{-}}+\int_{x_{b}^{+}}^{L}\right)\left(-\frac{1}{2} h u^{3}-g h^{2} u\right)_{x} \mathrm{~d} x-S\left[\frac{1}{2} h u^{2}+\frac{1}{2} g h^{2}\right] \\
= & {\left[\frac{1}{2} h u^{3}+g h^{2} u\right]-S\left[\frac{1}{2} h u^{2}+\frac{1}{2} g h^{2}\right], }
\end{aligned}
$$


which equals expression (13.86) in [154]. Using the jump conditions [2.71, the expression 2.72 takes the known form

$$
\frac{\mathrm{d} \mathcal{H}}{\mathrm{d} t}=\frac{g\left(h^{+}-h^{-}\right)^{3} h^{-}\left(S-u^{-}\right)}{4 h^{-} h^{+}},
$$

which means that if $h^{+}>h^{-}$and $S-u^{+}<0$ or $h^{+}<h^{-}$and $S-u^{+}>0$ energy is lost, in 2.73). These cases can also be clarified by taking the velocity $u^{-}=0$, keeping the left domain at rest, when the bore comes. When $h^{+}>h^{-}$, the bore then must come from the right, and therefore $S<0$, whence the condition is satisfied. Vice versa when $u^{+}=0$, the right domain is at rest. When $h^{+}<h^{-}$, then the bore must come from the left and $S>0$.

\subsubsection{D Jump conditions for new water wave model}

The jump conditions for the new water wave model in two horizontal dimensions (2DH) can be obtained in a similar way. The three dimensional domain $\Omega$ is split into two parts: $\Omega_{1}$ lying on one side of the bore and $\Omega_{2}$ is lying on the other side, see Fig. 2.6 Viewed from above, the maximum horizontal extents of these two domains at the free surface are denoted by $\Omega_{1 H}$ and $\Omega_{2 H}$, respectively. Between the domains there is a vertical and curved moving interface $\partial \Omega_{b}$, corresponding to the unknown bore position $\left(x_{b}, y_{b}\right)(t)$ in the horizontal plane. The domain $\Omega$ is taken to have solid wall boundaries and a flat bottom. The free surface $z=h$ is denoted as $\partial \Omega_{s}$. Assume that $h^{-}>h^{+}$with $h^{-}$and $h^{+}$along the interface $\partial \Omega_{b}$ in $\Omega_{1}$ and $\Omega_{2}$, respectively. Hence, the bore moves towards $\Omega_{2}$ with speed $S=\left(\dot{x}_{b}, \dot{y}_{b}\right)^{T} \cdot \vec{n}$. We use $\vec{n}$ for the outward normal of the domain $\Omega_{1}$ at the point $\mathbf{x}_{b}=\left(x_{b}, y_{b}\right)^{T}$ along the moving boundary $\partial \Omega_{b}$. At the same point $\mathbf{x}_{b}$ the outward normal $\vec{n}_{2}$ in $\Omega_{2}$ has the opposite sign: $\vec{n}_{2}=-\vec{n}$. In the expressions that follow, we generally omit the third zero component of this normal at the vertical boundary $\partial \Omega_{b}$.

The velocity field in the new water wave model has the form

$$
\vec{U}(x, y, z, t)=\nabla \phi+\vec{\pi}_{i} \nabla \overrightarrow{l_{i}}=\nabla \phi+\vec{v}=\vec{u}^{*}+\nabla \varphi,
$$

with $i=1,2$. The depth-averaged horizontal velocity is defined as

$$
\overline{\vec{u}}(x, y, t)=\frac{1}{h} \int_{0}^{h} \vec{U}_{H} \mathrm{~d} z .
$$


As before in (2.2), the variational principle for the new water wave model has the form

$$
\begin{aligned}
0 & =\delta \int_{0}^{T} \mathcal{L}\left[\vec{l}, \vec{\pi}, \phi, \phi_{s}, h\right] \mathrm{d} t \\
& =\delta \int_{0}^{T}\left(\int_{\Omega_{1 H}} \int_{0}^{h}\left(\partial_{t} \phi+\vec{\pi} \cdot \partial_{t} \vec{l}+\frac{1}{2}|\vec{U}|^{2}+g\left(z-H_{0}\right)\right) \mathrm{d} z \mathrm{~d} x \mathrm{~d} y\right. \\
+ & \left.\int_{\Omega_{2 H}}^{h}\left(\partial_{0} \phi+\vec{\pi} \cdot \partial_{t} \vec{l}+\frac{1}{2}|\vec{U}|^{2}+g\left(z-H_{0}\right)\right) \mathrm{d} z \mathrm{~d} x \mathrm{~d} y\right) \mathrm{d} t .
\end{aligned}
$$

First, we have to identify the independent variables, with respect to which we take the variations. Clearly, these include $\phi$ in the interior, and $h, \phi_{s}, \vec{l}$ and $\vec{\pi}$ at the free surface. Again, we impose the following continuity assumptions on the velocity potential $\phi_{b}=\phi\left(x_{b}, y_{b}, z, t\right)=\phi\left(x_{b}^{-}, y_{b}^{-}, z, t\right)=\phi\left(x_{b}^{+}, y_{b}^{+}, z, t\right)$ and the particle labels $\vec{l}_{b}=\vec{l}\left(x_{b}, y_{b}, t\right)=\vec{l}\left(x_{b}^{-}, y_{b}^{-}, t\right)=\vec{l}\left(x_{b}^{+}, y_{b}^{+}, t\right)$ at the bore boundary $\partial \Omega_{b}$. It turns out later that $\vec{l}_{b}$ and $\phi_{b}$ emerge as independent variations as well. The interior boundary is evolving in time, implying that $x_{b}(q, t)$ and $y_{b}(q, t)$ are part of the dynamics for some parameterization involving $q$ along $\partial \Omega_{b}$. It turns out to be more convenient to work in a coordinate system along $\partial \Omega_{b}$ that is aligned with the normal vector $\vec{n}$ and tangential vector $\vec{\tau}$ tangential to it, such that $\vec{n} \cdot \vec{\tau}=0$. By definition this normal coincides with the direction of the jump with speed $S$. Instead of $\delta \mathbf{x}_{b}$ we will use $\left(\delta n_{b}, \delta \tau_{b}\right)^{T}$, concerning the variations of a bore position in the $\vec{n}$ and $\vec{\tau}$ directions. The projection of the vector $\delta \mathbf{x}_{b}$ on the normal $\vec{n}$ is $\delta n_{b}=\left(\delta x_{b}, \delta y_{b}\right)^{T} \cdot \vec{n}$. Similary, $\delta \tau_{b}=\left(\delta x_{b}, \delta y_{b}\right)^{T} \cdot \vec{\tau}$, with $\vec{n}=\left(n_{1}, n_{2}\right)^{T}$ and $\vec{\tau}=\left(\tau_{1}, \tau_{2}\right)^{T}$ the unit vectors of the new coordinate system. Hence, we find

$$
\begin{aligned}
& \delta x_{b}=n_{1} \delta n_{b}+\tau_{1} \delta \tau_{b}, \\
& \delta y_{b}=n_{2} \delta n_{b}+\tau_{2} \delta \tau_{b} .
\end{aligned}
$$

Using (2.77), we relate variations of $\phi$ at the boundary $\partial \Omega_{b}$, as follows

$$
\begin{aligned}
& \delta\left(\phi_{b}\right)=(\delta \phi)_{b}+\left(\phi_{x}\right)_{b} \delta x_{b}+\left(\phi_{y}\right)_{b} \delta y_{b} \\
& =(\delta \phi)_{b}+\left(n_{1} \phi_{x}+n_{2} \phi_{y}\right)_{b} \delta n_{b}+\left(\tau_{1} \phi_{x}+\tau_{2} \phi_{y}\right)_{b} \delta \tau_{b} \\
& =(\delta \phi)_{b}+(\boldsymbol{\nabla} \phi \cdot \vec{n})_{b} \delta n_{b}+(\boldsymbol{\nabla} \phi \cdot \vec{\tau})_{b} \delta \tau_{b} .
\end{aligned}
$$

Similar formulas can be obtained, as follows

$$
\begin{aligned}
& \delta\left(h^{+}\right)=(\delta h)^{+}+(\nabla h)^{+} \cdot \vec{n} \delta n_{b}+(\nabla h)^{+} \cdot \vec{\tau} \delta \tau_{b}, \\
& \frac{\mathrm{d} h^{+}}{\mathrm{d} t}=\left(h_{t}\right)^{+}+(\nabla h)^{+} \cdot \vec{n} S, \\
& \frac{\mathrm{d} \phi_{b}}{\mathrm{~d} t}=\left(\phi_{t}\right)_{b}+(\nabla \phi)_{b} \cdot \vec{n} S .
\end{aligned}
$$


The subdomains $\Omega_{i}$ with $i=1,2$ are time-dependent, since the interface between these subdomains moves in time. We therefore have to use a variational analogue of Reynolds transport theorem (see, e.g., [35, 49], and Appendix 2.7.2], as follows

$$
\delta \int_{\Omega_{i}} F \mathrm{~d} x \mathrm{~d} y \mathrm{~d} z=\int_{\partial \Omega_{i}} F \delta \mathbf{x}_{\Gamma} \cdot \vec{n}_{\Gamma} \mathrm{d} \Gamma_{i}+\int_{\Omega_{i}} \delta F \mathrm{~d} x \mathrm{~d} y \mathrm{~d} z
$$

with the time-dependent part of the boundary $\Gamma_{i}, \vec{n}_{\Gamma}$ the three dimensional normal to the boundary, and $\delta \mathbf{x}_{\Gamma}=\left(\delta x_{\Gamma}, \delta y_{\Gamma}, \delta z_{\Gamma}\right)^{T}$ the variations of the coordinates of that boundary. Given 2.76, the expression for $F$ is complicated and depends on the independent variables in the variational principle. It may in principle also contain given functions of space, such as the bottom topography $b=b(x, y)$ (here set to zero for simplicity). There are two time dependent parts of the subdomains $\Omega_{i}$ in 2.76): the 2D free surface $\Gamma_{s}$ and the bore boundary $\partial \Omega_{b}$, which extends from $z \in[0, h]$ along the horizontal 1D bore line $\Gamma_{b}$. The outward normal at the free surface is $\mathbf{n}_{s}=\left(-h_{x},-h_{y}, 1\right)^{T} / \sqrt{1+\left(h_{x}\right)^{2}+\left(h_{y}\right)^{2}}$. The chosen parameterization is $z-h(x, y, t)=0$ for a single-valued free surface, in which $x$ and $y$ are the coordinates. In addition, $\mathrm{d} \Gamma_{s}=\sqrt{1+\left(h_{x}\right)^{2}+\left(h_{y}\right)^{2}} \mathrm{~d} x \mathrm{~d} y$. Hence,

$$
\begin{aligned}
\delta \mathbf{x}_{s} \cdot \vec{n}_{s} \mathrm{~d} \Gamma_{s} & =\left(\delta x_{s} \delta y_{s}, \delta z_{s}\right)^{T} \cdot\left(-h_{x},-h_{y}, 1\right) \mathrm{d} x \mathrm{~d} y \\
& =\delta h \mathrm{~d} x \mathrm{~d} y
\end{aligned}
$$

since $\delta x_{s}=\delta x=0, \delta y_{s}=\delta y=0$ and $\delta z_{s}=\delta h$. The bore boundary is vertical and a line when viewed from above with parameterization $x_{b}(q, t)$ and $y_{b}(q, t)$ with parameter $q$ along this line. The tangential vector $\vec{\tau}=\left(x_{q}, y_{q}, 0\right)^{T}$ with $x_{q} \equiv \partial_{q} x_{b}$ and $y_{q} \equiv$ $\partial_{q} y_{b}$. Hence the three-dimensional normal is $\vec{n}=\left(-y_{q}, x_{q}, 0\right)^{T}$ in the direction of bore propagation. Consequently, Reynolds' theorem for variations used here becomes

$$
\delta \int_{\Omega_{i}} F \mathrm{~d} x \mathrm{~d} y \mathrm{~d} z=\int_{\Gamma_{s}} F \delta h \mathrm{~d} x \mathrm{~d} y+\int_{0}^{h} \int_{\Gamma_{b}} F \delta \mathbf{x}_{b} \cdot \vec{n}_{b} \mathrm{~d} \Gamma_{b} \mathrm{~d} z+\int_{\Omega_{i}} \delta F \mathrm{~d} x \mathrm{~d} y \mathrm{~d} z
$$

with $\mathrm{d} \Gamma_{b}$ an infinitesimal line element along the bore line $\Gamma_{b}$.

Reynolds' transport theorem for time derivatives has a similar form as 2.82) provided we change the variational derivatives by time derivatives, giving:

$$
\frac{\mathrm{d}}{\mathrm{d} t} \int_{\Omega_{i}} F \mathrm{~d} x \mathrm{~d} y \mathrm{~d} z=\int_{\Gamma_{s}} F \partial_{t} h \mathrm{~d} x \mathrm{~d} y+\int_{0}^{h} \int_{\Gamma_{b}} F S \mathrm{~d} \Gamma_{b} \mathrm{~d} z+\int_{\Omega_{i}} \partial_{t} F \mathrm{~d} x \mathrm{~d} y \mathrm{~d} z .
$$


Application of (2.82) to the variations in 2.76 yields

$$
\begin{aligned}
& 0=\int_{0}^{T} \int_{\Omega_{1 H}, \Omega_{2 H}}\left(\partial_{t} \phi+\vec{\pi} \cdot \partial_{t} \vec{l}+\frac{1}{2}|\vec{U}|^{2}+g\left(h-H_{0}\right)\right)_{s} \delta h \mathrm{~d} x \mathrm{~d} y \\
& -\int_{\Gamma_{b}}\left[\int_{0}^{h}\left(\partial_{t} \phi+\vec{\pi} \cdot \partial_{t} \vec{l}+\frac{1}{2}|\vec{U}|^{2}\right) \mathrm{d} z+\frac{g h^{2}}{2}-g h H_{0}\right] \delta n_{b} \mathrm{~d} \Gamma_{b} \\
& +\int_{\Omega_{1 H}, \Omega_{2 H}} \int_{0}^{h} \delta \phi_{t}+\vec{U} \cdot \delta(\nabla \phi)+\vec{\pi}_{i} \vec{U} \cdot \delta\left(\nabla \overrightarrow{l_{i}}\right) \\
& \quad+\vec{U} \cdot \nabla \vec{l}_{i} \delta \vec{\pi}_{i} \mathrm{~d} z+h \vec{l}_{t} \cdot \delta \vec{\pi}+h \vec{\pi} \cdot \delta \vec{l}_{t} \mathrm{~d} x \mathrm{~d} y \mathrm{~d} t,
\end{aligned}
$$

with the jump notation $[F]=F^{+}-F^{-}$for some arbitrary quantity $F$ across the bore.

We illustrate the derivation by working out one of the variations in detail. Using Reynolds' transport theorems 2.82) and 2.83, the variation of the integrals involving $\phi_{t}$ in 2.76) (see also 2.84) becomes

$$
\begin{gathered}
\delta \int_{0}^{T} \int_{\Omega_{1 H}, \Omega_{2 H}} \int_{0}^{h} \phi_{t} \mathrm{~d} z \mathrm{~d} x \mathrm{~d} y \mathrm{~d} t=\int_{0}^{T} \int_{\Gamma_{s}}\left(\phi_{t}\right)_{s} \delta h \mathrm{~d} x \mathrm{~d} y+\int_{\Gamma_{b}} \int_{0}^{h}\left[-\phi_{t}\right] \delta n_{b} \mathrm{~d} z \mathrm{~d} \Gamma_{b} \\
+\frac{d}{d t} \int_{\Omega_{1 H}, \Omega_{2 H}} \int_{0}^{h} \delta \phi \mathrm{d} z \mathrm{~d} x \mathrm{~d} y-\int_{\Gamma_{s}} h_{t}(\delta \phi)_{s} \mathrm{~d} x \mathrm{~d} y-\int_{\Gamma_{b}} S\left[\int_{0}^{h}(\delta \phi)_{b} \mathrm{~d} z\right] \mathrm{d} \Gamma_{b} \mathrm{~d} t .
\end{gathered}
$$

In the last step, we use the end-point conditions $(\delta \phi)_{t=0}=(\delta \phi)_{t=T}=0$, expression 2.78 for $(\delta \phi)_{b}$ and a similar expression for $(\delta \phi)_{s}=\delta\left(\phi_{s}\right)-\left(\phi_{z}\right)_{s} \delta h$, in order to determine the variations with respect to the independent variables. The variations in the last two terms in 2.85) thus become

$$
\begin{aligned}
& =\int_{0}^{T}-\int_{\Gamma_{s}} h_{t}\left(\delta\left(\phi_{s}\right)-\left(\phi_{z}\right)_{s} \delta h\right) \mathrm{d} x \mathrm{~d} y \\
& -\int_{\Gamma_{b}}\left[S \int_{0}^{h}\left(\delta\left(\phi_{b}\right)-\nabla \phi \cdot \vec{n} \delta n_{b}-\nabla \phi \cdot \vec{\tau} \delta \tau_{b}\right) \mathrm{d} z\right] \mathrm{d} \Gamma_{b} \mathrm{~d} t .
\end{aligned}
$$

Analyzing the variations of 2.84 in a similar way, while integrating by parts, using the endpoint conditions $(\delta \phi)_{t=0}=(\delta \phi)_{t=T},(\delta \vec{l})_{t=0}=(\delta \vec{l})_{t=T}$, and the definition of the 
velocity (2.74), we first obtain the following system of equations

$$
\begin{aligned}
\delta \phi: & \nabla^{2} \phi+\nabla \cdot \vec{v}=0 \quad \text { in } \Omega \backslash \partial \Omega_{b}, \\
\delta h: \quad & \partial_{t} \phi_{s}+\frac{1}{2}\left|\nabla_{H} \phi_{s}+\vec{v}\right|^{2}+g\left(h-H_{0}\right)-\vec{v} \cdot \overrightarrow{\vec{u}} \\
& -\frac{1}{2}\left(\partial_{z} \phi\right)_{s}^{2}\left(1+\left|\nabla_{H} h\right|^{2}\right)=0 \quad \text { in } \Gamma_{s} \backslash \Gamma_{b}, \\
\delta \phi_{s}: \quad & \partial_{t} h-\left(\partial_{z} \phi\right)_{s}\left(1+\left|\nabla_{H} h\right|^{2}\right) \\
& +\left(\nabla_{H} \phi_{s}+\vec{v}\right) \cdot \nabla_{H} h=0 \quad \text { in } \Gamma_{s} \backslash \Gamma_{b}, \\
\delta(h \vec{\pi}): & \partial_{t} \vec{l}+\overrightarrow{\vec{u}} \cdot \nabla \vec{l}=0 \quad \text { in } \Gamma_{s} \backslash \Gamma_{b}, \\
\delta \vec{l}: & \partial_{t}(h \vec{\pi})+\nabla \cdot(h \overline{\vec{u}} \vec{\pi})=0 \text { in } \Gamma_{s} \backslash \Gamma_{b},
\end{aligned}
$$

similar to 2.7) and 2.9. It can also be reformulated to 2.28 and 2.29.

Second, the variations with respect to the interior boundary variables at $\partial \Omega_{b}$ arise with the help of relations 2.78, 2.79, and equations 2.87):

$$
\begin{aligned}
\delta \phi_{b}: & {[h(\overline{\vec{u}} \cdot \vec{n}-S)]=0, } \\
\delta n_{b}: & {\left[\int_{0}^{h}(\vec{U} \cdot \vec{n})(\vec{U} \cdot \vec{n}-S) \mathrm{d} z-\frac{1}{2} g h^{2}-\int_{0}^{h}\left(\phi_{t}+\frac{1}{2}|\vec{U}|^{2}\right) \mathrm{d} z+h \vec{v} \cdot \overrightarrow{\vec{u}}\right]=0, } \\
& \left.\quad \int_{0}^{h}(\vec{U} \cdot \vec{\tau})(\vec{U} \cdot \vec{n}-S) \mathrm{d} z\right]=0, \\
\delta \tau_{b}: & \quad\left[\vec{l}_{b}: \quad[h \vec{\pi}(\overline{\vec{u}} \cdot \vec{n}-S)]=0 .\right.
\end{aligned}
$$

Together with 2.88a, condition 2.88d expresses continuity of the Lagrange multipliers $\vec{\pi}$. The jump conditions $2.88 \mathrm{a}, 2.88 \mathrm{~b}$ ) coincide with the jump conditions resulting from the conservative form 2.34 of the new water wave model, and the jump condition 2.88c shows that the tangential component of velocity contains no jump.

The expected loss of energy can be found via a similar procedure as for the shallow water equations. Taking the time derivative of the Hamiltonian, invoking Reynolds' 
theorem (2.83), and using (2.87) extensively, we find

$$
\begin{aligned}
\frac{\mathrm{d} \mathcal{H}}{\mathrm{d} t} & =\frac{\mathrm{d}}{\mathrm{d} t} \int_{\Omega_{1 H}, \Omega_{2 H}}\left(\int_{0}^{h} \frac{1}{2}|\vec{U}|^{2} \mathrm{~d} z+\frac{1}{2} g h^{2}\right) \mathrm{d} x \mathrm{~d} y \\
& =\int_{\Omega_{1 H}, \Omega_{2 H}}\left(\int_{0}^{h} \frac{1}{2}|\vec{U}|^{2} \mathrm{~d} z+\frac{1}{2} g h^{2}\right)_{t} \mathrm{~d} x \mathrm{~d} y-\int_{\Gamma_{b}} S\left[\int_{0}^{h} \frac{1}{2}|\vec{U}|^{2} \mathrm{~d} z+\frac{1}{2} g h^{2}\right] \mathrm{d} \Gamma_{b} \\
& =-\int_{\Gamma_{b}}\left[\int_{0}^{h} \vec{U} \cdot \vec{n} \phi_{t} \mathrm{~d} z+(h \overline{\vec{u}} \cdot \vec{n})(\overrightarrow{\mathrm{v}} \cdot \overrightarrow{\vec{u}})\right] \mathrm{d} \Gamma_{b}-\int_{\Gamma_{b}} S\left[\int_{0}^{h} \frac{1}{2}|\vec{U}|^{2} \mathrm{~d} z+\frac{1}{2} g h^{2}\right] \mathrm{d} \Gamma_{b} .
\end{aligned}
$$

Additionally, we used a rewritten form of the continuity equation $\partial_{t} h+\nabla \cdot(h \overline{\vec{u}})=0$ and $\partial_{t} \vec{v}+\overline{\vec{u}} \cdot \nabla \vec{v}+\vec{v} \nabla \overline{\vec{u}}=0$. Using jump conditions 2.88, expression 2.89) finally takes the form

$$
\frac{\mathrm{d} \mathcal{H}}{\mathrm{d} t}=\int_{\Gamma_{b}} Q \mathrm{~d} \Gamma_{b} \equiv \int_{\Gamma_{b}}\left[\int_{0}^{h}(S-\vec{U} \cdot \vec{n})\left(\phi_{t}+S(\vec{U} \cdot \vec{n})-\overrightarrow{\mathrm{v}} \cdot \overrightarrow{\vec{u}}\right) \mathrm{d} z\right] \mathrm{d} \Gamma_{b}
$$

with rate of energy loss $Q$ along the bore boundary.

\subsubsection{D Jump conditions for shallow water equations}

The variational approach can be implemented for the $2 \mathrm{D}$ shallow water equations. Nevertheless, the final result for the jump conditions coincides with the jump conditions derived from 2.88) under the simplification of the velocity potential. When we assume the velocity potential to be $\phi=\phi_{s}$, the shallow water velocity field emerges as

$$
\begin{aligned}
\vec{U}(x, y, t) & =\overrightarrow{\vec{u}}=\vec{u}^{*}(x, y, t)=\nabla \phi_{s}(x, y, t)+\vec{v}(x, y, t) \\
& =\nabla \phi_{s}+\vec{\pi}_{i} \nabla \vec{l}_{i},
\end{aligned}
$$

with $i, j=1,2$. For the surface velocity potential $\phi_{z}=0$, which allows us to compute the integral in 2.88b explicitly and to simplify equation 2.87b. We substitute 2.87b into 2.88b), which leads to the Rankine-Hugoniot conditions for the shallow water equations at $\Gamma_{b}$ :

$$
\begin{array}{ll}
\delta \phi_{b}: & {[h(\overline{\vec{u}} \cdot \vec{n}-S)]=0,} \\
\delta n_{b}: & {\left[h(\overline{\vec{u}} \cdot \vec{n})(\overline{\vec{u}} \cdot \vec{n}-S)+\frac{1}{2} g h^{2}\right]=0,} \\
\delta \tau_{b}: & {[h(\overline{\vec{u}} \cdot \vec{\tau})(\overline{\vec{u}} \cdot \vec{n}-S)]=0,} \\
\delta \vec{l}_{b}: & {[h \vec{\pi}(\overline{\vec{u}} \cdot \vec{n}-S)]=0,}
\end{array}
$$


yielding again continuity of the Lagrange multipliers $\vec{\pi}$.

Under the assumption that $\phi=\phi_{s}$, while using the simplified version of 2.87b), the energy loss expression 2.90 reduces to the shallow water energy loss expression as follows

$$
Q=\left[(\overline{\vec{u}} \cdot \vec{n})\left(\frac{1}{2} h|\overline{\vec{u}}|^{2}+g h^{2}\right)\right]-S\left[\frac{1}{2} h|\overline{\vec{u}}|^{2}+\frac{1}{2} g h^{2}\right] .
$$

Using jump conditions 2.92a and 2.92b expression 2.93 takes the well-known form [121]

$$
Q=\frac{g\left(h^{+}-h^{-}\right)^{3} h^{+}\left(S-\vec{n} \cdot \overline{\vec{u}}^{+}\right)}{4 h^{-} h^{+}}
$$

which means that in the cases $h^{+}>h^{-}$and $\left(S-\vec{n} \cdot \overline{\vec{u}}^{+}\right)<0$ or $h^{+}<h^{-}$and $(S-\vec{n}$. $\left.\overline{\vec{u}}^{+}\right)>0$ the energy is lost. It is a natural 2D generalization of relation (2.73).

\subsubsection{D Jump conditions for new water wave model}

In one horizontal dimension the velocity field reduces to potential flow $\vec{U}=\nabla \phi$ with $\vec{v}=0$. We again split the domain $\Omega$ into two parts, $\Omega_{1}$ and $\Omega_{2}$ and in each of them the free surface profile is assumed single-valued.

The jump conditions 2.88a and 2.88b in $1 \mathrm{D}$ are reformulated as

$$
\begin{aligned}
{[h(S-\bar{u})] } & =0, \\
{\left[\int_{0}^{h}\left(\frac{1}{2} u^{2}-\frac{1}{2} w^{2}-\partial_{t} \phi\right) \mathrm{d} z-h \bar{u} S-\frac{1}{2} g h^{2}\right] } & =0,
\end{aligned}
$$

with vertical velocity component $w=\phi_{z}$.

It is worth to mention that the jump conditions could also be obtained from 2.34). In $1 \mathrm{D}$ the depth-averaged momentum equation in 2.34 takes the form:

$$
\left(\int_{0}^{h} u \mathrm{~d} z\right)_{t}+\left(\int_{0}^{h}\left(\frac{1}{2} u^{2}-\frac{1}{2} w^{2}-\partial_{t} \phi\right) \mathrm{d} z-\frac{1}{2} g h^{2}\right)_{x}=0
$$

which relates to the jump condition 2.95b).

The energy loss relation can be reduced from 2.90 to a form

$$
\frac{\mathrm{d} \mathcal{H}}{\mathrm{d} t}=\left[\int_{0}^{h}(S-u)\left(\phi_{t}+S u\right) \mathrm{d} z\right]
$$

It is not clear what the sign of $\mathrm{d} \mathcal{H} / \mathrm{d} t$ is in the previous expression. The integral expressions can be simplified when we use the Ritz method to approximate the velocity potential, which is illustrated next. 


\subsubsection{D Jump conditions for variational Boussinesq and Green-Naghdi equations}

When we substitute the Green-Naghdi Ansatz

$$
\phi=\phi_{s}+\frac{1}{2}\left(h^{2}-z^{2}\right) \psi
$$

into jump condition 2.95b), we obtain

$$
\left[h \bar{u}^{2}-h S \bar{u}+\frac{1}{2} g h^{2}-\frac{h^{3}}{3}\left(\psi_{t}-\psi^{2}+\bar{u} \psi_{x}\right)+\beta \frac{h^{5}}{15} \psi_{x}^{2}\right]=0 .
$$

after using that $\partial_{t} u^{*}=\partial_{t x}\left(\phi_{s}\right)=-B_{x}$ such that we can use $\partial_{t}\left(\phi_{s}\right)=-B$ with $B$ given in 2.58b. To reconstruct the Green-Naghdi system we take $\beta=0$ and following 2.59a take $\psi=\bar{u}_{x}$. Then jump conditions 2.95a), 2.99) are simplified as

$$
\begin{aligned}
{[h(S-\bar{u})] } & =0, \\
{\left[h \bar{u}^{2}-h S \bar{u}+\frac{g h^{2}}{2}-\frac{h^{3}}{3}\left(\bar{u}_{x t}-\left(\bar{u}_{x}\right)^{2}+\bar{u} \bar{u}_{x x}\right)\right] } & =0 .
\end{aligned}
$$

Considering 2.97 with the velocity potential simplified as 2.98 and for $\beta=0$, we reformulate the entropy expression for energy loss as follows

$$
\frac{\mathrm{d} \mathcal{H}}{\mathrm{d} t}=\left[h(\bar{u}-S)\left(\frac{(\bar{u}-S)^{2}}{2}+g h+\frac{h^{2} \bar{u}_{x}^{2}}{2}-\frac{h^{2}}{3}\left(\bar{u}_{x t}+\bar{u} \bar{u}_{x x}\right)\right)\right] .
$$

In order to check whether energy is lost in the jump we need to determine the sign of [2.101). The analysis of [42] shows that due to the strong influence of dispersion in the Green-Naghdi model a discontinuity cannot be maintained. In that case, 2.101 reduces to $\mathrm{d} \mathcal{H} / \mathrm{d} t=0$ as there is no discontinuity. It is unclear at the moment whether the variational Boussinesq model for the case $\beta=1$ can maintain bores. This can in principle be checked in a numerical model with a shock fitting approach, such that numerical dissipation at the discontinuity is at least avoided. Such an investigation is left to future work possibly using results from [2].

\subsection{Conclusions}

A systematic derivation of a new Hamiltonian formulation for water waves was given starting from the variational principle (2.2). The new water wave model includes both water-wave dispersion and the vertical component of the vorticity by construction. It was pointed out in [18] that the shallow water equations, or any Boussinesq system without proper circulation in the vertical plane, miss an instability they found in Benney's shallow water equations. It remains an open question to what extent this omission of horizontal vorticity components matters in the shallow water flows investigated here. 


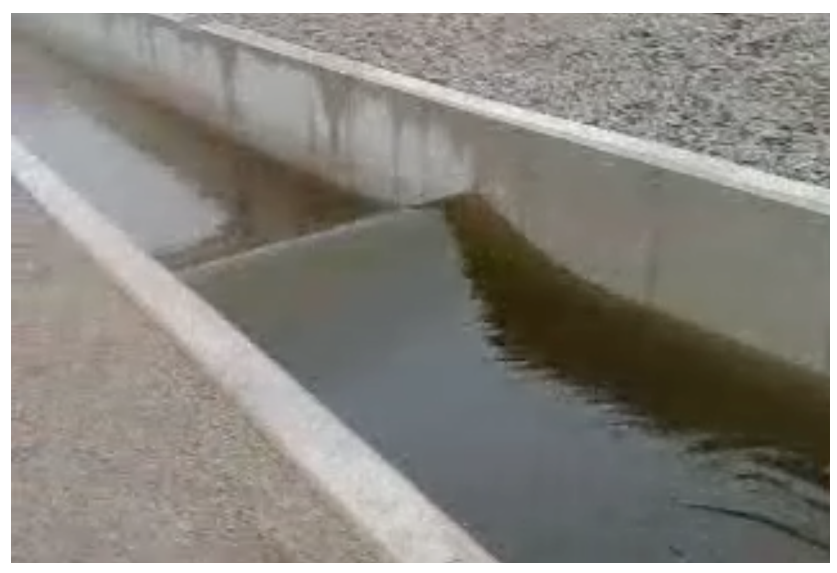

Figure 2.1: Bore-soliton-splash experiment: a smooth soliton is generated just after the sluice gate has been opened. Run case 6 [160].

Subsequently, we showed how the new Hamiltonian formulation reduces to the classical shallow water and potential flow models. The new system could be simpified further to an extension of the variational Boussinesq models of Klopman [82], now including potential vorticity. For a parabolic potential flow profile, these Hamiltonian Boussinesq models were shown to contain the Green-Naghdi system provided the velocity in the Hamiltonian was approximated further to be columnar.

Finally, a new variational approach to analyse systems with discontinuities was explored. It resulted into known jump conditions for the shallow water system and novel conditions for our new system. Moreover, it provides an apparatus to analyse the stability of shocks or jumps for systems with the Hamiltonian structure. We were, however, unable to determine yet whether the jumps derived for the new system and its Boussinesq simplifications could be sustained via local dissipation of energy in the bore. Future plans therefore include the numerical evaluation of these jump conditions including shock-fitting methods, in contrast to shock-capturing methods in which artificial, numerical disperion may incorrectly lead to smoothening of flows with discontinuities or bores. 


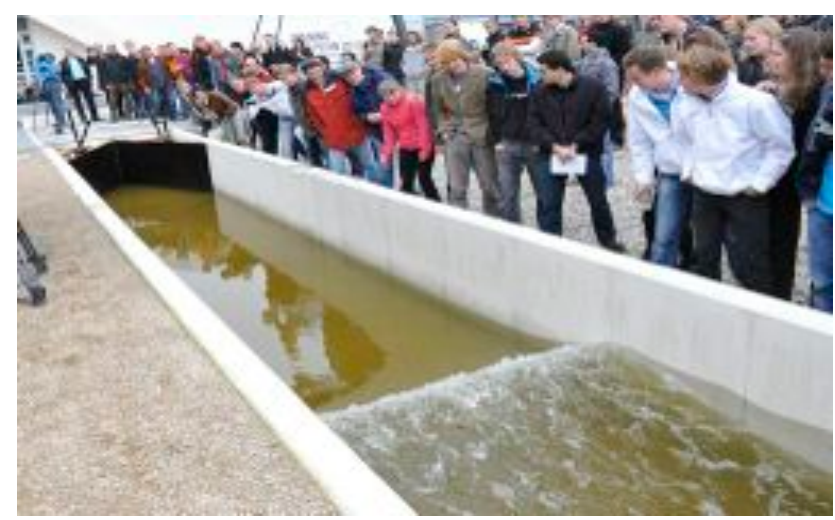

Figure 2.2: Bore-soliton-splash experiment: after the soliton breakes it propagates as a bore through the channel. Run case 8. Photo: University of Twente [13].

\subsection{Appendix}

\subsubsection{Integrals}

In this Appendix, we define the integrals in expressions 2.46-2.50, as follows

$$
\begin{aligned}
& F(h, b)=\int_{b}^{b+h} f^{2} \mathrm{~d} z, \quad G(h, b)=\int_{b}^{b+h}\left(\partial_{h} f\right)^{2} \mathrm{~d} z, \\
& K(h, b)=\int_{b}^{b+h}\left(\partial_{z} f\right)^{2} \mathrm{~d} z, \quad P(h, b)=\int_{b}^{b+h} f \mathrm{~d} z, \\
& Q(h, b)=\int_{b}^{b+h}\left(\partial_{h} f\right) \mathrm{d} z, \quad R(h, b)=\int_{b}^{b+h} f\left(\partial_{h} f\right) \mathrm{d} z .
\end{aligned}
$$

When $f(z ; b, h)$ equals the parabolic vertical profile 2.51, these integrals reduce to

$$
\begin{aligned}
& F=\frac{2}{15} h^{3}, \quad G=\frac{7}{15} h, \quad K=\frac{1}{3} h, \\
& P=-\frac{1}{3} h^{2}, \quad Q=-\frac{2}{3} h, \quad R=\frac{1}{5} h^{2} .
\end{aligned}
$$




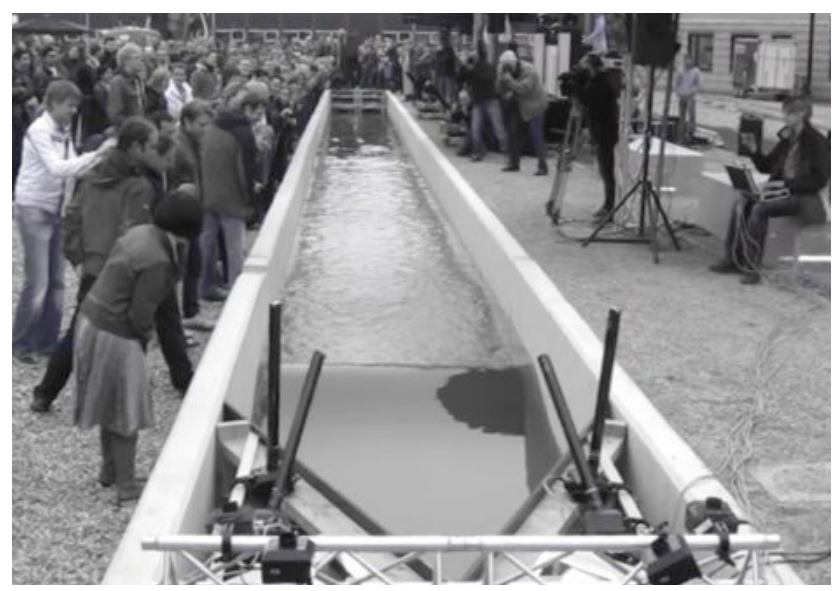

Figure 2.3: Bore-soliton-splash experiment: the broken wave has dissipated enough energy near the end of the channel such that it is smoothened back to a soliton of lower amplitude. Run case 8 [13].

\subsubsection{Variational Reynolds' transport theorem}

To take the variations of an integral with boundaries depending on dynamic variables, we have to obtain a variational analogue of Reynolds' transport theorem. Consider a domain $\Omega\left(x_{\Gamma}, y_{\Gamma}, z_{\Gamma}, t\right)$ in which (part of) the boundary $\partial \Omega_{\Gamma}$ is evolving in time. We need to find the variations of the integral

$$
I\left[F, \mathbf{x}_{\Gamma}\right]=\int_{\Omega\left(\mathbf{x}_{\Gamma}\right)} F(x, y, z, t) \mathrm{d} x \mathrm{~d} y \mathrm{~d} z,
$$

in which $F$ can depend implicitly on spatial coordinates and time via other variables, or an integral thereof, as in 2.76), or explicitly on $x, y, z$ and $t$. The variation has to be taken with respect to the function $F$ (in short, as in our case $F$ includes further dependencies) and the boundary positions $\mathbf{x}_{\Gamma}=\left(x_{\Gamma}, y_{\Gamma}, z_{\Gamma}\right)^{T}$. Taking a short-cut, the definition of the variation is

$$
\delta I=\lim _{\epsilon \rightarrow 0} \frac{1}{\epsilon}\left(I\left[F+\epsilon \delta F, \mathbf{x}_{\Gamma}+\epsilon \delta \mathbf{x}_{\Gamma}\right]-I\left[F, \mathbf{x}_{\Gamma}\right]\right) .
$$

We introduce a transformation $\chi: \hat{\Omega} \mapsto \Omega$ from reference space $\hat{\Omega}$ to physical space $\Omega$, assuming that such a transformation exist. The reference space has coordinates $\xi_{1}=$ $\xi, \xi_{2}=\eta$ and $\xi_{3}=\zeta$. The evaluation of $F$ in the reference space is denoted by $F \circ \chi \equiv$ $F(\vec{x}=\vec{\chi}(\xi, \eta, \zeta), t)$, which includes the complicated dependence on the variables, as 
discussed. The inverse of $\chi$ is denoted by $\chi^{-1}$ and is assumed to exist. It transforms the physical domain $\Omega$ into a reference domain $\hat{\Omega}$. The key simplification used is that the reference domain is fixed in time. We denote the Jacobian matrix of this transformation by

$$
J=\left(\begin{array}{lll}
x_{\xi} & x_{\eta} & x_{\zeta} \\
y_{\xi} & y_{\eta} & y_{\zeta} \\
z_{\xi} & z_{\eta} & z_{\zeta}
\end{array}\right)
$$

and its determinant as $|J|$.

The integral over the domain $\Omega$ is calculated to be

$$
\int_{\Omega} F(x, y, z, t) \mathrm{d} x \mathrm{~d} y \mathrm{~d} z=\int_{\hat{\Omega}} F \circ \chi|J| \mathrm{d} \xi_{1} \mathrm{~d} \xi_{2} \mathrm{~d} \xi_{3} .
$$

The variations are easier in reference space, given that the reference domain is fixed in time. We thus obtain

$$
\begin{aligned}
& \delta \int_{\hat{\Omega}} F \circ \chi|J| \mathrm{d} \xi_{1} \mathrm{~d} \xi_{2} \mathrm{~d} \xi_{3}=\int_{\hat{\Omega}} \delta(F \circ \chi|J|) \mathrm{d} \xi_{1} \mathrm{~d} \xi_{2} \mathrm{~d} \xi_{3} \\
= & \int_{\hat{\Omega}}|J| \delta(F \circ \chi)+(F \circ \chi) \delta|J| \mathrm{d} \xi_{1} \mathrm{~d} \xi_{2} \mathrm{~d} \xi_{3} .
\end{aligned}
$$

We consider the terms in 2.108 consecutively. The first one becomes

$$
\int_{\hat{\Omega}}|J| \delta(F \circ \chi) \mathrm{d} \xi_{1} \mathrm{~d} \xi_{2} \mathrm{~d} \xi_{3}=\int_{\Omega} \delta(F \circ \chi) \circ \chi^{-1} \mathrm{~d} x \mathrm{~d} y \mathrm{~d} z .
$$

To evaluate the second term in 2.108, we have to take variations of the Jacobian, as follows

$$
\delta|J|=\nabla_{\xi} \cdot\left(|J| J^{-1} \mathrm{w}\right)
$$

with the "variational wind" $\mathrm{w}=\delta \mathbf{x} \circ \chi$ denoting variations $\delta \mathbf{x}$ projected to the reference space, and $\nabla_{\xi} \equiv(\partial / \partial \xi, \partial / \partial \eta, \partial / \partial \zeta)^{T}$. This leads to

$$
\begin{aligned}
& \int_{\hat{\Omega}}(F \circ \chi) \delta|J| \mathrm{d} \xi_{1} \mathrm{~d} \xi_{2} \mathrm{~d} \xi_{3}=\int_{\hat{\Omega}}(F \circ \chi) \nabla_{\xi} \cdot\left(|J| J^{-1} \mathrm{w}\right) \mathrm{d} \xi_{1} \mathrm{~d} \xi_{2} \mathrm{~d} \xi_{3} \\
& =\int_{\hat{\Omega}} \nabla_{\xi} \cdot\left((F \circ \chi)|J| J^{-1} \mathrm{w}\right)-\nabla_{\xi}(F \circ \chi) \cdot\left(|J| J^{-1} \mathrm{w}\right) \mathrm{d} \xi_{1} \mathrm{~d} \xi_{2} \mathrm{~d} \xi_{3} .
\end{aligned}
$$


It is worthwhile noting that we use the general vector $\delta \mathbf{x}$ because it depends through the transformation $\chi$, in a generally complicated manner, on $\delta \mathbf{x}_{\Gamma}$. The dependency does not need to be found explicitly as will become clear shortly. Only at the moving parts of the boundary $\partial \Omega_{\Gamma}$ is $\delta \mathbf{x}=\delta \mathbf{x}_{\Gamma}$, in contrast to the situation at the other boundaries.

In 2.111, we consider the terms seperately. The first one can be evaluated using Gauss' theorem as follows

$$
\begin{aligned}
& \int_{\hat{\Omega}} \nabla_{\xi} \cdot\left((F \circ \chi)|J| J^{-1} \mathrm{w}\right) \mathrm{d} \xi_{1} \mathrm{~d} \xi_{2} \mathrm{~d} \xi_{3} \\
& =\int_{\partial \hat{\Omega}}\left((F \circ \chi)|J| J^{-1} \mathrm{w}\right) \cdot \vec{n}_{0} \mathrm{~d} \Gamma_{0}=\int_{\partial \Omega} F \delta \mathbf{x} \cdot \vec{n} \mathrm{~d} \Gamma
\end{aligned}
$$

with $\vec{n}_{0}, \mathrm{~d} \Gamma_{0}$ and $\vec{n}, \mathrm{~d} \Gamma$ the normal and line elements along the boundary in the reference and physical spaces, respectively. We note that $J \vec{n}=|J| \vec{n}_{0}$ follows directly from taking the gradient $\nabla_{\xi}$ of the equations of the same plane tangent to the surface at the boundary: $\vec{n} \cdot \mathbf{x}=C_{0}$ and $\vec{n}_{0} \cdot \boldsymbol{\xi}=C_{1}$ in the physical and references spaces. The actual constants $C_{0}$ and $C_{1}$ are unimportant and the determinant acts as a normalization.

The last term of (2.111) is evaluated by using

$$
\nabla(F \circ \chi)=\left(J^{T}\right)^{-1} \nabla_{\xi}(F \circ \chi),
$$

such that we obtain

$$
\int_{\hat{\Omega}} \nabla_{\xi}(F \circ \chi) \cdot\left(|J| J^{-1} \mathrm{w}\right) \mathrm{d} \xi_{1} \mathrm{~d} \xi_{2} \mathrm{~d} \xi_{3}=\int_{\Omega} \boldsymbol{\nabla}(F \circ \chi) \cdot \delta \mathbf{x} \mathrm{d} x \mathrm{~d} y \mathrm{~d} z .
$$

After all, we combine the results in 2.110 and 2.114) into:

$$
\begin{aligned}
& \delta \int_{\Omega} F(x, y, z, t) \mathrm{d} x \mathrm{~d} y \mathrm{~d} z=\int_{\partial \Omega} F \delta \mathbf{x} \cdot \vec{n} \mathrm{~d} \Gamma \\
& +\int_{\Omega} \delta(F \circ \chi) \circ \chi^{-1}-\nabla(F \circ \chi) \cdot \delta \mathbf{x} \mathrm{d} x \mathrm{~d} y \mathrm{~d} z,
\end{aligned}
$$

Using the chain rule for variations, one can derive that

$$
\int_{\Omega} \delta(F \circ \chi) \circ \chi^{-1} \mathrm{~d} x \mathrm{~d} y \mathrm{~d} z=\int_{\Omega} \nabla(F \circ \chi) \cdot \delta \mathbf{x}+(\delta F) \mathrm{d} x \mathrm{~d} y \mathrm{~d} z,
$$

such we can combine the last two terms of 2.115). Hence, we finally derived the required Reynolds' transport theorem for variations 2.80$]$ used in the main text

$$
\delta \int_{\Omega} F \mathrm{~d} x \mathrm{~d} y \mathrm{~d} z=\int_{\partial \Omega} F \delta \mathbf{x}_{\Gamma} \cdot \vec{n} \mathrm{~d} \Gamma+\int_{\Omega} \delta F \mathrm{~d} x \mathrm{~d} y \mathrm{~d} z,
$$

where we used $\left.\delta \mathbf{x}\right|_{\partial \Omega}=\delta \mathbf{x}_{\Gamma}$. 


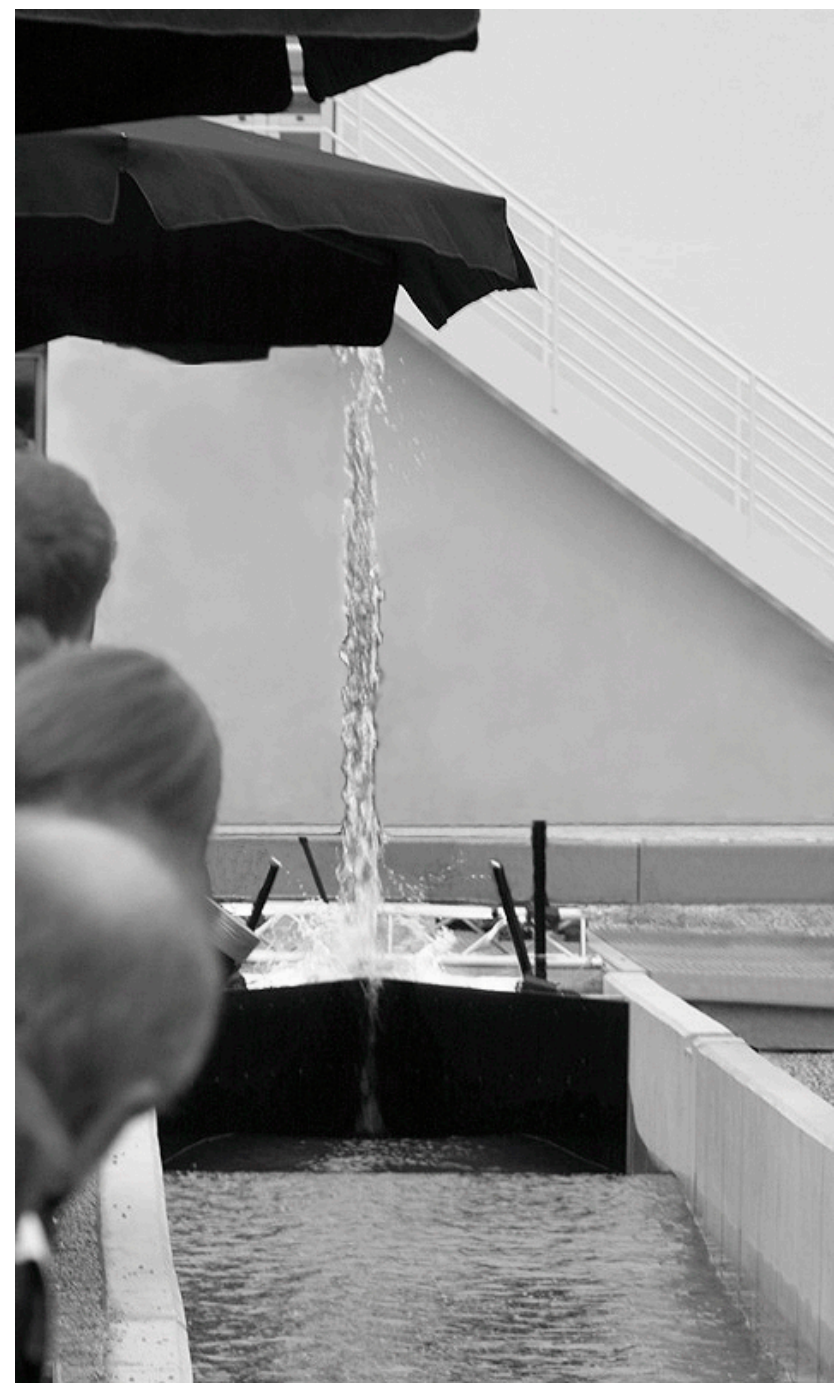

Figure 2.4: Bore-soliton-splash experiment: the final splash in the constriction of the channel. Run case 8 [160]. 


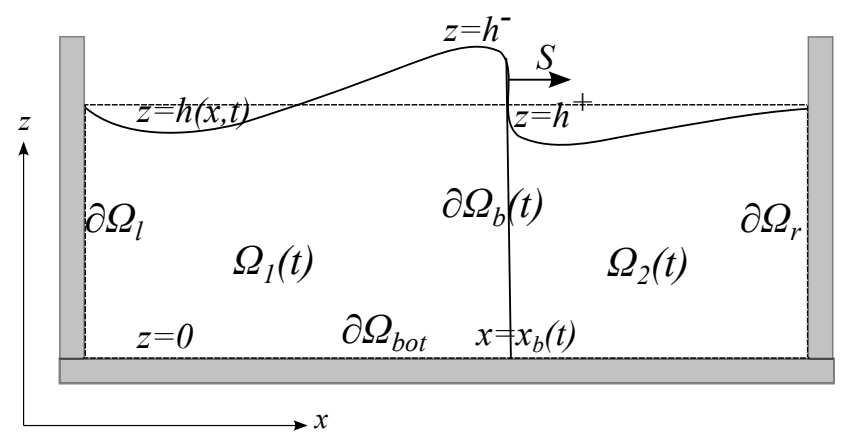

Figure 2.5: Domain sketch for a breaking wave. Vertical cut with axes $(x, z)$.

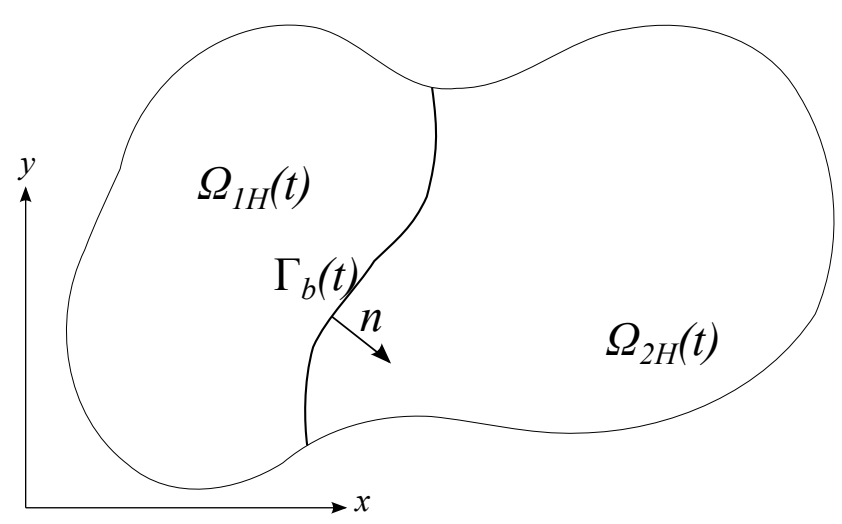

Figure 2.6: Domain sketch for a breaking wave. Horizontal cut with axes $(x, y)$. The bore boundary $\partial \Omega_{b}$ is a vertical sheet with bore line $\Gamma_{b}$. 


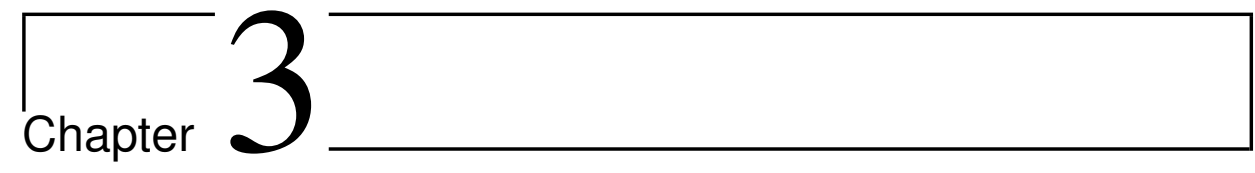

\section{Symplectic Time Discontinuous Galerkin Discretizations for Hamiltonian Systems}

Various systems in nature have a Hamiltonian structure and therefore accurate time integrators for those systems are of great practical use. In this chapter, a finite element method will be explored to derive symplectic time stepping schemes for (non)autonomous systems in a systematic way. The technique used is a variational discontinuous Galerkin finite element method in time. This approach provides a unified framework to derive known and new symplectic time integrators. An extended analysis for the new time integrators will be provided. This analysis shows that a novel third order time integrator presented in this chapter has excellent dispersion properties. Also, the time stepping schemes are proven to be accurate for non-autonomous systems 1 .

\subsection{Introduction}

The dynamics of various physical phenomena, such as the movement of pendulums, planets, or water waves can be described in a variational framework. The development of variational principles for classical mechanics traces back to Euler, Lagrange, and Hamilton; an overview of this history can be found in [6], [103]. This approach allows to express all the dynamics of a system in a single functional - the Lagrangian - which is an action integral. Hamiltonian mechanics is a reformulation of Lagrangian mechanics which

\footnotetext{
${ }^{1}$ The content of this chapter is joint work in collaboration with S. Nurijanyan, V. Ambati, J.J.W. van der Vegt and O. Bokhove, and will be submitted as journal articles.
} 
provides a convenient framework to study the symmetry properties of a system. This is expressed by Noether's theorem which establishes the direct connection between the symmetry properties of Hamiltonian systems and conservation laws. When one approximates the equations numerically, it is advantageous to preserve the Hamiltonian structure also at the discrete level. Given that Hamiltonian systems are abundant in nature, their numerical approximation is therefore a topic of significant relevance.

The topic of time integration of Hamiltonian systems has seen a rigorous development during the past decades. This has resulted in symplectic time integrators that have been designed to preserve the Hamiltonian structure for an approximated system. More information on symplectic integration of Hamiltonian systems can be found in [64] and [92]. According to [104], variational time integrators can be proven to be symplectic by construction under certain conditions. A review on variational time integration can be found in [57] and [104]. In the variational approach, one can derive well-known schemes or new schemes, as reported in [57], [93], [113] and [139].

One of the most actively developed numerical methods is the finite element method with time discontinuous basis functions. Such a discontinuous Galerkin method in time was studied by [38], [39] and [44]. In particular, the variational Galerkin finite element method in time was implemented by [61], [104] and [113]. Integrators for forced variational principles were developed among others in [104].

The study of the variational time integrators that are discussed in this chapter was motivated by our research into numerical discretizations of the equations describing nonlinear water waves, which have a Hamiltonian structure. The Hamiltonian structure of nonlinear gravity free surface water waves was shown by Zakharov [159]. In [52] (and Chapter 4 of this thesis), we constructed a numerical water wave tank based on a discrete Miles' variational principle with forcing. The computational method had to fulfill a number of requirements in order to perform the simulations of the laboratory experiments presented in [68]. First, the experiments with which we compared numerical results run over a long period of time, covering also an extensive spatial domain, which meant that conservation of mass and bounded fluctuations of energy at the discrete level are important. Second, in one of the test cases in [52] (and Chapter 4) a focussing wave was created with a wave height five times exceeding the ambient, incoming wave height. Therefore numerical stability had to be ensured. Third, a piston wave maker was used in the experiments, which gives external forcing to the system and results in a non-autonomous Hamiltonian system. It is non-trivial to meet all these requirements, in particular in combination with a spatial discretization of the nonlinear potential flow water equations. We therefore investigate variational time integrators in detail in this chapter.

In the construction of variational time integrators we choose to work with a discontinuous Galerkin finite element method in time because it allows additional freedom to develop new symplectic integrators, also for non-autonomous Hamiltonian systems. In this method the time domain is split into elements, and in each element the variables are approximated with polynomial expansions. The discrete variational principle is then obtained by substitution of the approximations in the continuum variational principle, 
provided one calculates the contribution of the delta functions that arise. After evaluation of the variational principle by calculating its variations, one obtains the discrete system of equations for the variational time integration method.

A crucial part of the discontinuous finite element method is the numerical flux arising from the before-mentioned delta functions. It has to be introduced to establish a connection between between the time elements since in each element only a local polynomial approximation is used. We study a specially chosen numerical flux, somewhat inspired by the one explored in [125] and [126], which in turn was based on the theory of nonconservative products developed in [34] and [147]. The flux is also implemented in the numerical discretization for the nonlinear potential flow water wave equations, discussed in Chapter 4 and gives excellent results.

Combining the discrete variational principle with the derived numerical flux, we obtain a unified approach to construct time integrators for (non)-autonomous Hamiltonian systems. We derive both well-known and novel symplectic time stepping schemes of first, second and third order accuracy. An extensive analysis for the discretizations is provided, including a linear stability analysis and an investigation of the symplectic nature of the schemes. The novel third order scheme is shown to have improved dispersion properties. Furthermore, within this approach we derive and test time stepping schemes for nonautonomous Hamiltonian systems, such as forced and damped oscillators. We study the approximation of forcing and damping terms considering the discrete versions of certain integrals.

The outline of this chapter is as follows. In Section 3.2 we introduce the dynamics of a Hamiltonian system with one degree of freedom. Next, Section 3.3 introduces the variational discontinuous Galerkin method. Here we obtain first and second order time stepping schemes, which can be formulated in the form of well-known symplectic schemes. In Section 3.4 two novel time integrators of second and third order accuracy in time are derived and analyzed. In Section 3.5 we present numerical results for nonautonomous systems. Conclusions are drawn in Sec. 3.6. More details on the analysis of the time integration methods are presented in the Appendix.

\subsection{Dynamics of a Hamiltonian system with one degree of freedom}

The dynamics of a Hamiltonian system with one degree of freedom is embedded in the Lagrangian functional

$$
\mathcal{L}(p, q, t):=\int_{0}^{T}\left(p \frac{\mathrm{d} q}{\mathrm{~d} t}-H(p, q)\right) \mathrm{d} t
$$

where $p(t)$ is the momentum, $q(t)$ the coordinate, and $H(p, q)$ the energy (or so-called Hamiltonian) of the system. 
The variational principle for the system is $\delta \mathcal{L}:=\delta_{p} \mathcal{L}+\delta_{q} \mathcal{L}=0$, where $\delta_{p} \mathcal{L}$ and $\delta_{q} \mathcal{L}$ are the functional derivatives with respect to $p$ and $q$, respectively. The functional derivatives are defined as

$$
\delta \mathcal{L}=\lim _{\epsilon \rightarrow 0} \frac{1}{\epsilon}(\mathcal{L}(p+\epsilon \delta p, q+\epsilon \delta q)-\mathcal{L}(p, q)) .
$$

Consider the variation of the Lagrangian functional 3.1

$$
\delta \mathcal{L}(p, q, t)=\int_{0}^{T}\left(\frac{\mathrm{d} q}{\mathrm{~d} t} \delta p+p \frac{\mathrm{d} \delta q}{\mathrm{~d} t}-\frac{\delta H}{\delta p} \delta p-\frac{\delta H}{\delta q} \delta q\right) \mathrm{d} t=0 .
$$

After integration by parts of 3.3 , using the end point conditions $\delta q(0)=\delta q(T)=0$, and the arbitrariness of the variations, the dynamics of a Hamiltonian system emerges as follows:

$$
\delta p: \frac{\mathrm{d} q}{\mathrm{~d} t}-\frac{\delta H}{\delta p}=0 \quad \text { and } \quad \delta q: \frac{\mathrm{d} p}{\mathrm{~d} t}+\frac{\delta H}{\delta q}=0,
$$

with initial conditions $p(t=0)=p_{0}$ and $q(t=0)=q_{0}$.

\subsection{Variational discontinuous Galerkin time discretiza- tion of a Hamiltonian system}

\subsubsection{Discrete functional}

The accurate numerical approximation of equations (3.4) is a well-developed subject of research. Geometric integrators target on the preservation of system properties at the discrete level and symplectic integrators ensure that the approximated system is also Hamiltonian, [64]. We start to approximate the Lagrangian (3.1) rather than approximating equations 3.4 .

To approximate the functional 3.1 for a single-degree-of-freedom system in time, we first divide the time domain $[0, T]$ into $N$ finite time intervals $I_{n}=\left(t_{n}, t_{n+1}\right), n=$ $0, \ldots, N-1$, with length $\Delta t=t_{n+1}-t_{n}$. Each time interval $I_{n}$ has a constant length and is related to a reference domain $\hat{I}=(-1,1)$ through the mapping $F_{n}$, defined as

$$
F_{n}: \hat{I} \rightarrow I_{n}: \tau \mapsto t=\frac{1}{2}\left(t_{n}(1-\tau)+t_{n+1}(1+\tau)\right) .
$$

Second, the functions $(p, q)$ are approximated element-wise with a polynomial expansion. In every element the basis functions are continuous, but could be discontinuous across element boundaries. The following finite element space is defined

$$
V^{\tau}:=\left\{v^{\tau}\left|v^{\tau} \in L^{2}([0, T]), v^{\tau}\right|_{I_{n}} \in P_{s}\left(I_{n}\right), \quad \forall I_{n}, n=0, \cdots, N-1\right\},
$$


where $P_{s}$ is the space of polynomials of degree $s$. Each variable in finite element $I_{n}$ is now approximated as $p^{\tau} \in V^{\tau}, q^{\tau} \in V^{\tau}$ and represented in a time slab $\left(t_{n}, t_{n+1}\right)$ as

$$
p^{\tau}=p^{i} \varphi^{i}, \quad q^{\tau}=q^{i} \psi^{i},
$$

with basis functions $\varphi^{i}, \psi^{i}$, which do not necessarily have to coincide, and expansion coefficients $p^{i}, \psi^{i}$. In this thesis, we will consider in particular Lagrange and Bernstein polynomials. Also, the Einstein summation convention will be used, which implies summation over repeating indices.

In order to account for the discontinuities at the element boundary we introduce the right and left traces of a variable $d(t)$ at time $t_{n}$ as

$$
d^{n,+}:=\lim _{\epsilon \downarrow 0} d\left(t_{n}+\epsilon\right) \quad \text { and } \quad d^{n,-}:=\lim _{\epsilon \downarrow 0} d\left(t_{n}-\epsilon\right),
$$

respectively. Then at each time level $t_{n}$ the following jump [[. $\left.]\right]$ and average $\{\{\cdot\}\}_{\alpha}^{\beta}$ operators can be defined

$$
\left.[[d]]\right|_{t_{n}}=d^{n,-}-d^{n,+} \quad \text { and }\left.\quad\{\{d\}\}_{\alpha}^{\beta}\right|_{t_{n}}=\alpha d^{n,-}+\beta d^{n,+},
$$

where $\alpha, \beta$ are arbitrary real values with $\alpha+\beta=1$ and $\alpha, \beta \geq 0$. The discrete functional for the Hamiltonian system, defined in 3.1 , is now obtained after introducing the polynomial approximations of $p$ and $q$ into the functional and the splitting of the time integral into a summation over time intervals

$$
\begin{aligned}
\mathcal{L}^{\tau}\left(p^{\tau}, q^{\tau}, t\right):= & \sum_{n=0}^{N-1} \int_{t_{n}}^{t_{n+1}}\left(p^{\tau} \frac{\mathrm{d} q^{\tau}}{\mathrm{d} t}-H\left(p^{\tau}, q^{\tau}\right)\right) \mathrm{d} t \\
& -\left.\sum_{n=-1}^{N-1}\left[\left[q^{\tau}\right]\right]\left\{\left\{p^{\tau}\right\}\right\}_{\alpha}^{\beta}\right|_{t_{n+1}} .
\end{aligned}
$$

The last term in 3.10 comes as a numerical flux ${ }^{2}$. The flux is required to establish a connection between the time element boundaries, as we use discontinuous basis functions.

There is still some freedom in the definition of the discrete Lagrangian 3.10). In the first place we need to specify the polynomial order of the basis functions. As in general it is not possible or desirable to compute the integral of the Hamiltonian analytically, we also have to choose a quadrature rule [104]. In [65] it is proven that the order of the quadrature rule will give an upper bound for the attainable order of the time integration scheme. Finally, the choice of the weight $\alpha$ in the flux term also leaves some freedom to derive and optimize different time integration methods.

The derivation of a time integration scheme is now performed as follows. We choose a polynomial approximation of order $s$. To obtain a scheme of order $s+1$ we should take

\footnotetext{
${ }^{2}$ Personal communication Vijaya Ambati and Onno Bokhove, who suggested and derived these flux terms, loosely inspired by the flux terms for non-conservative products in hyperbolic systems [125], [126].
} 


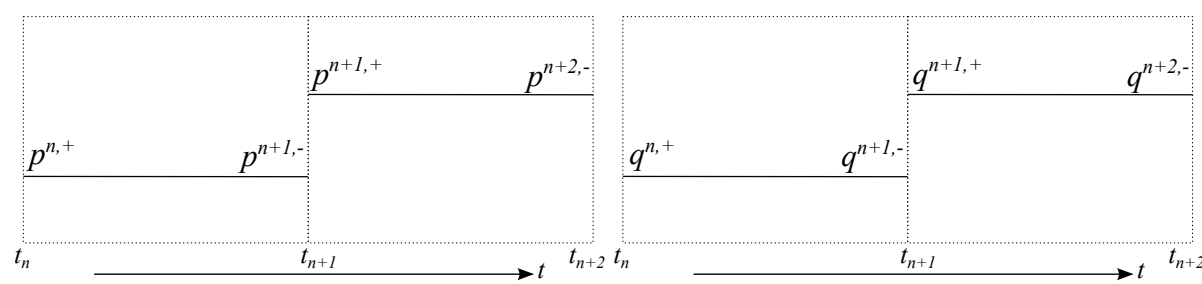

Figure 3.1: Piecewise constant approximation in time.

a quadrature rule with an order of accuracy that is at least $s+1$, [65]. After the scheme is derived, a linear stability analysis will be performed to find appropriate weights $\alpha$ in the flux term. For novel schemes further analysis will be performed to define the computational properties of the time integration scheme.

\subsubsection{First order variational time discretization}

We will now show that several well-known symplectic time integration schemes can be derived directly from the discrete variational formulation 3.10. To obtain a first order time integrator we choose constant basis functions $\left(p^{\tau}, q^{\tau}\right)=\left(p^{n,+}, q^{n,+}\right)$ in each time interval $I_{n}$. As we may see from the approximation and also Figure 3.1, then we have $p^{n,+}=p^{n+1,-}$. For a piece-wise constant approximation of the variables a one point quadrature approximation

$$
\int_{t_{n}}^{t_{n+1}} H\left(p^{\tau}, q^{\tau}\right) \mathrm{d} t=\Delta t H\left(p^{n,+}, q^{n,+}\right)
$$

will be exact. Next, we substitute (3.11) into the discrete functional 3.10 . Since $q^{\tau}=$ const in each time element, the term $p^{\tau} \frac{\mathrm{d} q^{\tau}}{\mathrm{d} t}$ drops out. The discrete functiona $\sqrt[3]{3}$ then becomes

$$
\begin{aligned}
\mathcal{L}^{\tau}\left(p^{\tau}, q^{\tau}, t\right)= & -\sum_{n=0}^{N-1} H\left(p^{n,+}, q^{n,+}\right) \Delta t \\
& -\sum_{n=-1}^{N-1}\left(q^{n,+}-q^{n+1,+}\right)\left(\alpha p^{n,+}+\beta p^{n+1,+}\right) .
\end{aligned}
$$

\footnotetext{
${ }^{3}$ Investigation of this variational principle for $\alpha=0$ and $\alpha=1$ was done by V. Ambati and O. Bokhove.
} 
Applying the variational principle $\delta \mathcal{L}^{\tau}=0$ and using what is called "discrete integration by parts" (for the rearranging of the summation, see [104]), we get

$$
\begin{aligned}
& \sum_{n=0}^{N-1}\left(-\frac{\partial H\left(p^{n,+}, q^{n,+}\right)}{\partial p^{n,+}} \delta p^{n,+}-\frac{\partial H\left(p^{n,+}, q^{n,+}\right)}{\partial q^{n,+}} \delta q^{n,+}\right) \Delta t \\
& +\sum_{n=-1}^{N-1}\left(\left(-\alpha\left(q^{n,+}-q^{n+1,+}\right)-\beta\left(q^{n-1,+}-q^{n,+}\right)\right) \delta p^{n,+}\right. \\
& \left.+\left(-\alpha p^{n,+}-\beta p^{n+1,+}+\alpha p^{n-1,+}+\beta p^{n,+}\right) \delta q^{n,+}\right)=0 .
\end{aligned}
$$

Now using the arbitrariness of the variations $\delta q^{n,+}$ and $\delta p^{n,+}$, and boundary conditions $\delta q^{0,-}=0, \delta p^{0,-}=0, \delta q^{N,+}=0, \delta p^{N,+}=0$, we obtain the following first order time stepping scheme:

$$
\begin{array}{ll}
\delta q^{n,+}: & \beta p^{n+1,+}=(\beta-\alpha) p^{n,+}+\alpha p^{n-1,+}-\Delta t \frac{\partial H\left(p^{n,+}, q^{n,+}\right)}{\partial q^{n,+}}, \\
\delta p^{n,+}: & \alpha q^{n+1,+}=(\alpha-\beta) q^{n,+}+\beta q^{n-1,+}+\Delta t \frac{\partial H\left(p^{n,+}, q^{n,+}\right)}{\partial p^{n,+}} .
\end{array}
$$

This scheme is stable in three cases: when $\alpha=0, \alpha=0.5$, or $\alpha=1$. For $\alpha=0.5$ one could recover a modified symplectic midpoint scheme for a double time step $2 \Delta t$. With $\alpha=1, \beta=0$, the system becomes

$$
\begin{array}{ll}
\delta q^{n,+}: & p^{n,+}=p^{n-1,+}-\Delta t \frac{\partial H\left(p^{n,+}, q^{n,+}\right)}{\partial q^{n,+}}, \\
\delta p^{n,+}: & q^{n+1,+}=q^{n,+}+\Delta t \frac{\partial H\left(p^{n,+}, q^{n,+}\right)}{\partial p^{n,+}} .
\end{array}
$$

To obtain a known form for scheme 3.15 , we notice that by construction $p^{n,+}=p^{n+1,-}=$ : $p^{n+1}$. Introducing also the notation $q^{n}:=q^{n,+}$, we reformulate the equations as

$$
\begin{aligned}
& p^{n+1}=p^{n}-\Delta t\left(\frac{\partial H\left(p^{n+1}, q^{n}\right)}{\partial q^{n}}\right), \\
& q^{n+1}=q^{n}+\Delta t\left(\frac{\partial H\left(p^{n+1}, q^{n}\right)}{\partial p^{n+1}}\right),
\end{aligned}
$$

which is the well-known symplectic Euler scheme, [64]. To obtain an adjoint symplectic Euler method, one can choose $\alpha=0$ and $\beta=1$, and rename the variables in the approximation as $\left(p^{\tau}, q^{\tau}\right)=\left(p^{n}, q^{n+1}\right)$.

\subsubsection{Second order variational time discretization: Störmer-Verlet}

The next step is to obtain known schemes of second order accuracy. To obtain a second order time integrator we need to choose linear basis functions in the polynomial 

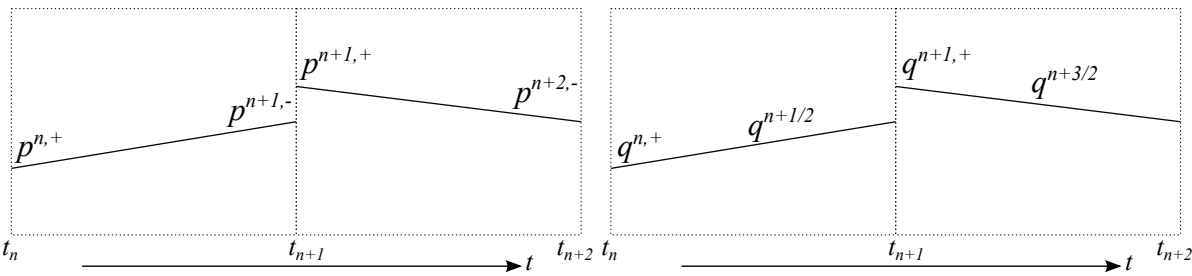

Figure 3.2: Piecewise linear approximation in time.

expansions. Various options for the approximation of the variables and integrals are possible. Since we want to derive a particular known Störmer-Verlet scheme, we take the trapezoidal rule for $p$ and the midpoint rule for $q$

$$
\int_{t_{n}}^{t_{n+1}} H\left(p^{\tau}, q^{\tau}\right) \mathrm{d} t=\frac{\Delta t}{2}\left(H\left(p^{n,+}, q^{n+1 / 2}\right)+H\left(p^{n+1,-}, q^{n+1 / 2}\right)\right),
$$

where the expansion coefficient $q^{n+1 / 2}$ is the value in the middle of the time interval. We approximate the variables in each time element $I_{n}$ as

$$
\begin{aligned}
& p^{\tau}=\frac{t^{n+1}-t}{\Delta t} p^{n,+}+\frac{t-t^{n}}{\Delta t} p^{n+1,-}, \\
& q^{\tau}=\frac{2\left(t-t^{n}\right)}{\Delta t} q^{n+1 / 2}+\frac{t^{n}+t^{n+1}-2 t}{\Delta t} q^{n,+},
\end{aligned}
$$

as shown at Figure 3.2 Introducing $3.177-\sqrt{3.18}$ ) in the discrete functional 3.10 we obtain $4^{4}$

$$
\begin{aligned}
\mathcal{L}^{\tau}\left(p^{\tau}, q^{\tau}, t\right)= & \sum_{n=0}^{N-1}\left(\left(p^{n,+}+p^{n+1,-}\right)\left(q^{n+1 / 2}-q^{n,+}\right)\right. \\
& \left.-\frac{\Delta t}{2}\left(H\left(p^{n,+}, q^{n+1 / 2}\right)+H\left(p^{n+1,-}, q^{n+1 / 2}\right)\right)\right) \\
& -\sum_{n=-1}^{N-1}\left(2 q^{n+1 / 2}-q^{n,+}-q^{n+1,+}\right)\left(\alpha p^{n+1,-}+\beta p^{n+1,+}\right) .
\end{aligned}
$$

Applying the variational principle $\delta \mathcal{L}^{\tau}=0$, using the arbitrariness of the variations, and using boundary conditions $\delta\left(2 q^{-1 / 2}-q^{-1,+}\right):=\delta q^{0,-}=0, \delta p^{0,-}=0, \delta q^{N,+}=$

\footnotetext{
${ }^{4}$ Investigation of this variational principle for $\alpha=0$ and $\alpha=1$ was done by V. Ambati and O. Bokhove.
} 

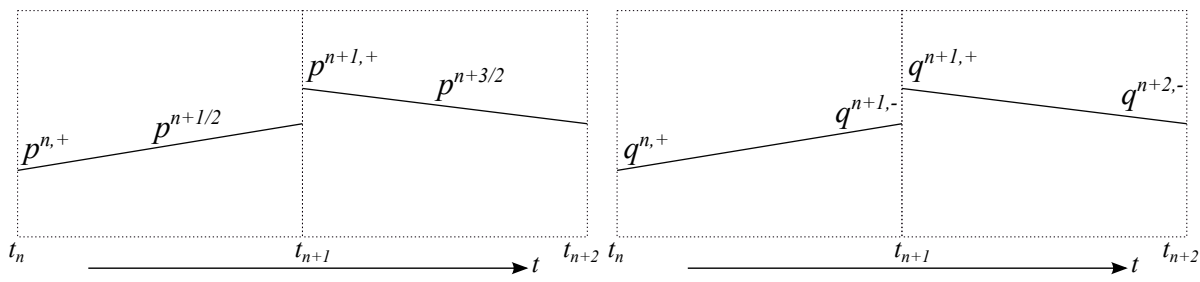

Figure 3.3: Piecewise linear approximation in time.

$0, \delta p^{N,+}=0$; we obtain the following variational time integration method

$$
\begin{aligned}
\delta q^{n,+}: \quad & (\beta-1) p^{n,+}+(\alpha-1) p^{n+1,-}+\beta p^{n+1,+}+\alpha p^{n,-}=0, \\
\delta p^{n,+}: \quad & q^{n+1 / 2}=q^{n,+}+\beta\left(2 q^{n-1 / 2}-q^{n-1,+}-q^{n,+}\right) \\
& +\frac{\Delta t}{2} \frac{\partial H\left(p^{n,+}, q^{n+1 / 2}\right)}{\partial p^{n,+}}, \\
\delta q^{n+1 / 2}: \quad & 2 \beta p^{n+1,+}+(2 \alpha-1) p^{n+1,-}=p^{n,+} \\
& -\frac{\Delta t}{2}\left(\frac{\partial H\left(p^{n,+}, q^{n+1 / 2}\right)}{\partial q^{n+1 / 2}}+\frac{\partial H\left(p^{n+1,-}, q^{n+1 / 2}\right)}{\partial q^{n+1 / 2}}\right), \\
\delta p^{n+1,-}: \quad & q^{n+1 / 2}-\alpha\left(2 q^{n+1 / 2}-q^{n,+}-q^{n+1,+}\right)=q^{n,+} \\
& +\frac{\Delta t}{2} \frac{\partial H\left(p^{n+1,-}, q^{n+1 / 2}\right)}{\partial p^{n+1,-}} .
\end{aligned}
$$

In Appendix 3.7.2 we show that the scheme is stable for weights $\alpha \in[0.5,1]$ and coincides under this choice with the well-known form of the second order Störmer-Verlet time integrator. It is interesting to note that we start with two discontinuous variables, but at the end there is no jump in $p$ at the time levels $t_{n}, n=0, \ldots, N$ anymore. Moreover, the presence of a jump in the $q$ variable does not result in a scheme different from the classical one (see Appendix 3.7.2.

An adjoint Störmer-Verlet scheme can be obtained via a swapped linear approximation of the variables in each element $I_{n}$

$$
\begin{aligned}
& q^{\tau}=\frac{t^{n+1}-t}{\Delta t} q^{n,+}+\frac{t-t^{n}}{\Delta t} q^{n+1,-}, \\
& p^{\tau}=\frac{2\left(t-t^{n}\right)}{\Delta t} p^{n+1 / 2}+\frac{t^{n}+t^{n+1}-2 t}{\Delta t} p^{n,+},
\end{aligned}
$$

and a trapezoidal rule for $q$ and a midpoint rule for $p$

$$
\int_{t_{n}}^{t_{n+1}} H\left(p^{\tau}, q^{\tau}\right) \mathrm{d} t=\frac{\Delta t}{2}\left(H\left(p^{n+1 / 2}, q^{n,+}\right)+H\left(p^{n+1 / 2}, q^{n+1,-}\right)\right) .
$$




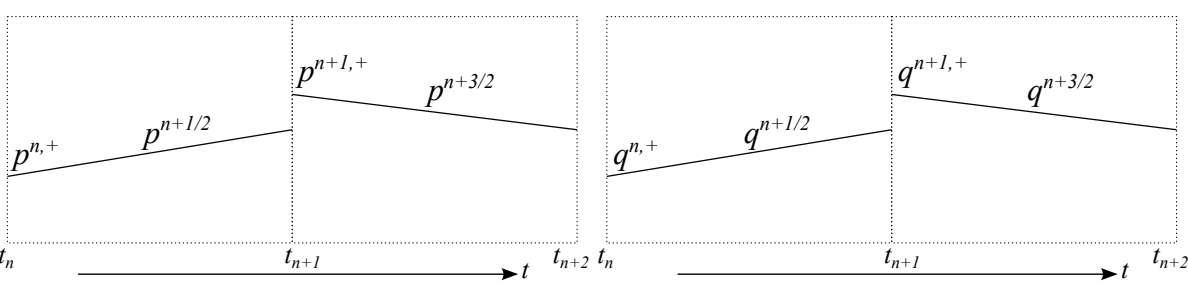

Figure 3.4: Piecewise linear approximation in time.

This weighted scheme is stable for $\alpha \in[0,0.5]$ and a reformulation allows to recover a well known formulation of the scheme, as given in [64].

\subsubsection{Second order time discretization: Modified symplectic mid- point scheme}

Next, we investigate a symmetric approximation of the variables using piecewise linear polynomials. This will result in the symplectic modified midpoint scheme, [64].

The variables $p^{\tau}$ and $q^{\tau}$ are approximated in the time slab $\left(t^{n}, t^{n+1}\right)$ as follows

$$
\begin{aligned}
& p^{\tau}=\frac{2\left(t-t^{n}\right)}{\Delta t} p^{n+1 / 2}+\frac{t^{n}+t^{n+1}-2 t}{\Delta t} p^{n,+} \text { and } \\
& q^{\tau}=\frac{2\left(t-t^{n}\right)}{\Delta t} q^{n+1 / 2}+\frac{t^{n}+t^{n+1}-2 t}{\Delta t} q^{n,+} .
\end{aligned}
$$

The integral of the Hamiltonian is approximated with the midpoint rule

$$
\int_{t_{n}}^{t_{n+1}} H\left(p^{\tau}, q^{\tau}\right) \mathrm{d} t \cong \Delta t H\left(p^{n+1 / 2}, q^{n+1 / 2}\right) .
$$

The discrete functional 3.10 then results in the following expression ${ }^{5}$

$$
\begin{gathered}
\mathcal{L}^{\tau}\left(p^{\tau}, q^{\tau}\right)=\sum_{n=0}^{N-1}\left(2 p^{n+1 / 2}\left(q^{n+1 / 2}-q^{n,+}\right)-\Delta t H\left(p^{n+\frac{1}{2}}, q^{n+\frac{1}{2}}\right)\right) \\
-\sum_{n=-1}^{N-1}\left(2 q^{n+1 / 2}-q^{n,+}-q^{n+1,+}\right)\left(\alpha\left(2 p^{n+1 / 2}-p^{n,+}\right)+\beta p^{n+1,+}\right) .
\end{gathered}
$$

Applying the variational principle $\delta \mathcal{L}^{\tau}=0$, using the arbitrariness of the variations, and using boundary conditions $\delta\left(2 q^{-1 / 2}-q^{-1,+}\right):=\delta q^{0,-}=0, \delta\left(2 p^{-1 / 2}-p^{-1,+}\right):=$

\footnotetext{
${ }^{5}$ Investigation of this variational principle for $\alpha=0.5$ was done by S. Nurijanyan.
} 
$\delta p^{0,-}=0, \delta q^{N,+}=0, \delta p^{N,+}=0 ;$ we obtain the following system of equations

$$
\begin{aligned}
\delta p^{n+1 / 2}: \quad 2 q^{n+1 / 2}-2 \alpha\left(2 q^{n+1 / 2}-q^{n,+}-q^{n+1,+}\right) & \\
= & 2 q^{n,+}+\Delta t \frac{\partial H\left(p^{n+\frac{1}{2}}, q^{n+\frac{1}{2}}\right)}{\partial p^{n+\frac{1}{2}}}, \\
\delta p^{n,+}: \quad \alpha\left(2 q^{n+1 / 2}-q^{n,+}-q^{n+1,+}\right)=\beta\left(2 q^{n-1 / 2}-q^{n-1,+}-q^{n,+}\right), & -2\left(\alpha\left(2 p^{n+1 / 2}-p^{n,+}\right)+\beta p^{n+1,+}\right)+2 p^{n+1 / 2} \\
\delta q^{n+1 / 2}: \quad & -\Delta t \frac{\partial H\left(p^{n+\frac{1}{2}}, q^{n+\frac{1}{2}}\right)}{\partial q^{n+\frac{1}{2}}}, \\
\delta q^{n,+}: \quad & -2 p^{n+1 / 2}+\alpha\left(2 p^{n+1 / 2}-p^{n,+}\right)+\beta p^{n+1,+} \\
& +\alpha\left(2 p^{n-1 / 2}-p^{n-1,+}\right)+\beta p^{n,+}=0 .
\end{aligned}
$$

To make further analysis easier we reformulate system 3.26 in terms of the jumps

$$
\begin{aligned}
\delta p^{n+1 / 2}: & q^{n+1,-}=q^{n,+}+\left.2 \alpha[[q]]\right|_{t_{n+1}}+\Delta t \frac{\partial H\left(p^{n+\frac{1}{2}}, q^{n+\frac{1}{2}}\right)}{\partial p^{n+\frac{1}{2}}}, \\
\delta p^{n,+}: & \left.\alpha[[q]]\right|_{t_{n+1}}=\left.\beta[[q]]\right|_{t_{n}}, \\
\delta q^{n+1 / 2}: & p^{n+1,-}=p^{n,+}+\left.2 \beta[[p]]\right|_{t_{n+1}}-\Delta t \frac{\partial H\left(p^{n+\frac{1}{2}}, q^{n+\frac{1}{2}}\right)}{\partial q^{n+\frac{1}{2}}}, \\
\delta q^{n,+}: & \left.\beta[[p]]\right|_{t_{n+1}}=\left.\alpha[[p]]\right|_{t_{n}} .
\end{aligned}
$$

Consider equations $3.27 \mathrm{~b}$ and $3.27 \mathrm{~d}$. A jump - the difference between the left and the right traces of a function at a time level - should be bounded, otherwise an unbounded growth will lead to instability. To have an upper bound for the jump in $q$, i.e. to ensure that the jump $\left.[[q]]\right|_{t_{n+1}}$ is smaller than the jump $\left.[[q]]\right|_{t_{n}}$, we get the requirement from (3.27b that $\alpha \geq 0.5$, while for the jump in $p$ we get the requirement from $3.27 \mathrm{~d}$ ) that $\alpha \leq 0.5$. These two requirements contradict for all values of $\alpha$, except for $\alpha=0.5$. Besides, if we initially take $\left.[[d]]\right|_{t_{0}}=0$, with $d=p$ or $q$, the jump will not change in time due to Equations (3.27b) and (3.27d). Continuity in one variable is then enforced and not required for the other variable. Therefore to ensure stability, one needs to take one of the following options a) $\alpha=0.5$; or b) $\left.[[p]]\right|_{t_{0}} \equiv 0$ with the restriction $\alpha \leq 0.5$; or c) $\left.[[q]]\right|_{t_{0}} \equiv 0$ with the restriction $\alpha \geq 0.5$. In all three cases we obtain then a system that 


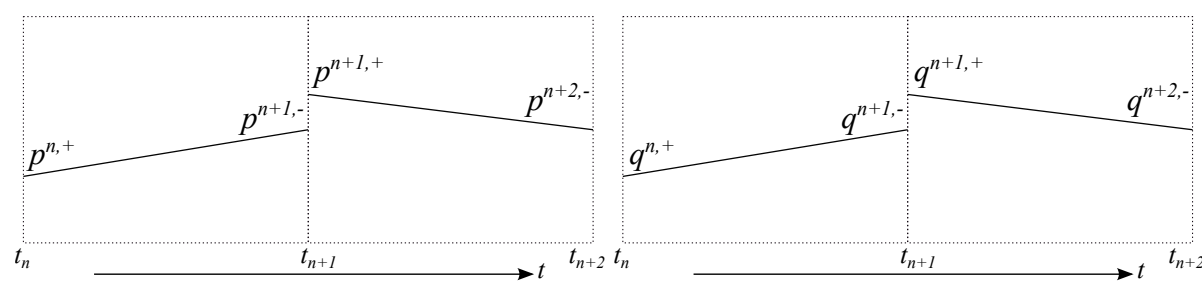

Figure 3.5: Piecewise linear approximation in time.

has the same structure as the symplectic modified midpoint scheme as given by [64], i.e.,

$$
\begin{aligned}
& p^{n+1}=p^{n}-\Delta t \frac{\partial H\left(p^{n+\frac{1}{2}}, q^{n+\frac{1}{2}}\right)}{\partial q^{n+\frac{1}{2}}}, \\
& q^{n+1}=q^{n}+\Delta t \frac{\partial H\left(p^{n+\frac{1}{2}}, q^{n+\frac{1}{2}}\right)}{\partial p^{n+\frac{1}{2}}} .
\end{aligned}
$$

To see that one has to introduce new variables in system (3.27). For convenience we can use initial conditions without a jump in the variables. Nevertheless, the equations allow an initial discontinuity in one of the variables to be present. The classical form of scheme (3.28) will be recovered by employing the notation $p^{n}:=\alpha p^{n,-}+\beta p^{n,+}$ if a jump is present in $p$, and $q^{n}:=\alpha q^{n,+}+\beta q^{n,-}$ if a jump is present in $q$. The values at the intermediate time level $t^{n+1 / 2}$ in the new notation are equal to $p^{n+1 / 2}:=\frac{1}{2}\left(p^{n}+p^{n+1}\right)$, and $q^{n+1 / 2}:=\frac{1}{2}\left(q^{n}+q^{n+1}\right)$. The introduction of the new variables is not necessary and can be avoided with the initial conditions $\left.[[p]]\right|_{t_{0}} \equiv 0$ and $\left.[[q]]\right|_{t_{0}} \equiv 0$.

\subsection{New variational time integrators}

A natural question is whether it is possible to find new time integrators following the variational approach described in the previous section. In this section (and Appendix 3.7.3 we will construct two new time integration schemes with second and third order accuracy using the variational approach. We will focus in this section on the quantitative analysis of the new schemes. In particular, we will investigate their convergence, symplecticity, linear stability and dispersion properties. 


\subsubsection{Explicit second order accurate time integration scheme}

An interesting case to investigate is the symmetric approximation of the variables in each time slab $I_{n}$ as

$$
\begin{aligned}
& p^{\tau}=\frac{t^{n+1}-t}{\Delta t} p^{n,+}+\frac{t-t^{n}}{\Delta t} p^{n+1,-}, \\
& q^{\tau}=\frac{t^{n+1}-t}{\Delta t} q^{n,+}+\frac{t-t^{n}}{\Delta t} q^{n+1,-},
\end{aligned}
$$

and a symmetric trapezoidal quadrature rule for the integral of the Hamiltonian is chosen

$$
\int_{t_{n}}^{t_{n+1}} H\left(p^{\tau}, q^{\tau}\right) \mathrm{d} t=\frac{\Delta t}{2}\left(H\left(p^{n,+}, q^{n,+}\right)+H\left(p^{n+1,-}, q^{n+1,-}\right)\right) .
$$

The discrete variational principle is given in Appendix 3.7 .3 by expression 3.100. The resulting scheme for $\alpha=0.5$ consists of four explicit steps

$$
\begin{aligned}
& q^{n+1,-}=q^{n,-}+\Delta t \frac{\partial H\left(p^{n,+}, q^{n,+}\right)}{\partial p^{n,+}}, \\
& p^{n+1,-}=p^{n,-}-\Delta t \frac{\partial H\left(p^{n,+}, q^{n,+}\right)}{\partial q^{n,+}}, \\
& q^{n+1,+}=q^{n,+}+\Delta t \frac{\partial H\left(p^{n+1,-}, q^{n+1,-}\right)}{\partial p^{n+1,-}}, \\
& p^{n+1,+}=p^{n,+}-\Delta t \frac{\partial H\left(p^{n+1,-}, q^{n+1,-}\right)}{\partial q^{n+1,-}},
\end{aligned}
$$

and is symplectic. The scheme works well for linear problems, but for the nonlinear water waves problem considered in this thesis it shows an increase in the energy error. Therefore the subject of applicability of the scheme requires further investigation, and we refer for details to Appendix 3.7.3. Some other applications of this scheme were considered by $\mathrm{O}$. Bokhove and available upon request. 


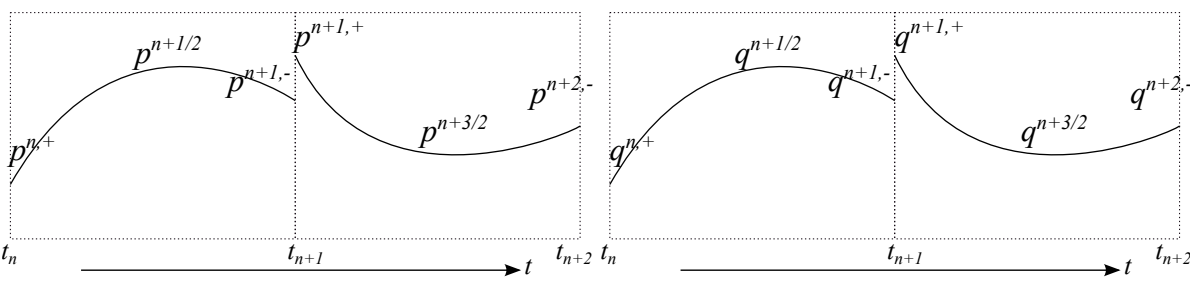

Figure 3.6: Piecewise quadratic approximation in time.

\subsubsection{Third order scheme}

To obtain a third order scheme we choose in each time element $I_{n}$ the following quadratic Lagrange basis functions

$$
\begin{aligned}
p^{\tau}= & \frac{\left(t^{n}+t^{n+1}-2 t\right)\left(t^{n+1}-t\right)}{\Delta t^{2}} p^{n,+}+\frac{4\left(t-t^{n}\right)\left(t^{n+1}-t\right)}{\Delta t^{2}} p^{n+1 / 2} \\
& -\frac{\left(t^{n}+t^{n+1}-2 t\right)\left(t-t^{n}\right)}{\Delta t^{2}} p^{n+1,-}, \\
q^{\tau}= & \frac{\left(t^{n}+t^{n+1}-2 t\right)\left(t^{n+1}-t\right)}{\Delta t^{2}} q^{n,+}+\frac{4\left(t-t^{n}\right)\left(t^{n+1}-t\right)}{\Delta t^{2}} q^{n+1 / 2} \\
& -\frac{\left(t^{n}+t^{n+1}-2 t\right)\left(t-t^{n}\right)}{\Delta t^{2}} q^{n+1,-},
\end{aligned}
$$

as can be seen in Fig. 3.6 There are three independent expansion coefficients present in the polynomial representation: $p^{n,+}$ at the left boundary of the interval, $p^{n+1 / 2}$ at the middle of the interval and $p^{n+1,-}$ at the right boundary of the interval. We approximate the integral over the Hamiltonian with Simpson's third order quadrature rule as

$$
\begin{aligned}
& \int_{t_{n}}^{t_{n+1}} H\left(p^{\tau}, q^{\tau}\right) \mathrm{d} t \\
& \quad \cong \frac{\Delta t}{6}\left(H\left(p^{n,+}, q^{n,+}\right)+4 H\left(p^{n+1 / 2}, q^{n+1 / 2}\right)+H\left(p^{n+1,-}, q^{n+1,-}\right)\right) .
\end{aligned}
$$


The discrete functional 3.10 then becomes

$$
\begin{aligned}
\mathcal{L}^{\tau}\left(p^{\tau}, q^{\tau}\right)= & \sum_{n=0}^{N-1} \int_{t^{n}}^{t^{n+1}} p^{\tau} \frac{\mathrm{d} q^{\tau}}{\mathrm{d} t} \mathrm{~d} t \\
& -\frac{\Delta t}{6}\left(H\left(p^{n,+}, q^{n,+}\right)+4 H\left(p^{n+1 / 2}, q^{n+1 / 2}\right)+H\left(p^{n+1,-}, q^{n+1,-}\right)\right) \\
& -\sum_{n=-1}^{N-1}\left(q^{n+1,-}-q^{n+1,+}\right)\left(\alpha p^{n+1,-}+\beta p^{n+1,+}\right) .
\end{aligned}
$$

This choice of quadrature rule and basis functions corresponds to the choices made in [113] for the scheme called P2N3Q4Lob. Nevertheless, the resulting scheme is different due to the choice of the numerical flux.

Applying the variational principle $\delta \mathcal{L}^{\tau}=0$, using the arbitrariness of the variations, and using boundary conditions $\delta q^{0,-}=0, \delta p^{0,-}=0, \delta q^{N,+}=0, \delta p^{N,+}=0$; we get a new third order scheme

$$
\begin{aligned}
& q^{n+1 / 2}=\left(1-\frac{3}{2} \beta\right) q^{n,+}+\frac{3}{2} \beta q^{n,-}+\frac{\Delta t}{4}\left(\frac{\partial H\left(p^{n,+}, q^{n,+}\right)}{\partial p^{n,+}}+\frac{\partial H\left(p^{n+1 / 2}, q^{n+1 / 2}\right)}{\partial p^{n+1 / 2}}\right) \\
& p^{n+1 / 2}=\left(1-\frac{3}{2} \alpha\right) p^{n,+}+\frac{3}{2} \alpha p^{n,-}-\frac{\Delta t}{4}\left(\frac{\partial H\left(p^{n,+}, q^{n,+}\right)}{\partial q^{n,+}}+\frac{\partial H\left(p^{n+1 / 2}, q^{n+1 / 2}\right)}{\partial q^{n+1 / 2}}\right), \\
& q^{n+1,-}=q^{n,+}+\Delta t \frac{\partial H\left(p^{n+1 / 2}, q^{n+1 / 2}\right)}{\partial p^{n+1 / 2}}, \\
& p^{n+1,-}=p^{n,+}-\Delta t \frac{\partial H\left(p^{n+1 / 2}, q^{n+1 / 2}\right)}{\partial q^{n+1 / 2}}, \\
& \alpha q^{n+1,+}=-\frac{1}{6} q^{n,+}+\frac{2}{3} q^{n+1 / 2}+\left(\alpha-\frac{1}{2}\right) q^{n+1,-}+\frac{\Delta t}{6} \frac{\partial H\left(p^{n+1,-}, q^{n+1,-}\right)}{\partial p^{n+1,-}}, \\
& \beta p^{n+1,+}=-\frac{1}{6} p^{n,+}+\frac{2}{3} p^{n+1 / 2}+\left(\beta-\frac{1}{2}\right) p^{n+1,-}-\frac{\Delta t}{6} \frac{\partial H\left(p^{n+1,-}, q^{n+1,-}\right)}{\partial q^{n+1,-}} .
\end{aligned}
$$

Equations (3.35a) and (3.35b) have to be solved together as an implicit system. The other four equations are explicit.

\section{Linear stability}

To perform the linear stability analysis we consider the Hamiltonian of the harmonic oscillator $H(p, q)=\frac{1}{2} \omega^{2} q^{2}+\frac{1}{2} p^{2}$ into scheme 3.35 . With the prescribed Hamiltonian 
we formulate scheme 3.35 in a form

$$
\left(p^{n+1,-}, q^{n+1,-}, p^{n+1,+}, q^{n+1,+}\right)^{T}=A\left(p^{n,-}, q^{n,-}, p^{n,+}, q^{n,+}\right)^{T} .
$$

To ensure that the numerical solution is bounded, it is required for the eigenvalues $\lambda_{i}$ of the matrix $A$ to be less than or equal to one in modulus [64]. The characteristic polynomial of the matrix $A$ is a quartic function in $\lambda$. Since, the explicit form of the eigenvalues is complicated we therefore compute the stability criteria numerically in Matlab. We take a range of weights $\alpha$, a range of frequencies times time step $\omega \triangle t$, substitute it to the expressions for the exact solutions of a quartic function, and show the domains where the maximum in modulus eigenvalue is smaller than one $\max _{i=1, \ldots, 4}\left|\lambda_{i}\right| \leq 1$. One can see in Figure 3.7 the only stability region located near $\omega \Delta t=0$ is the one corresponding to $\alpha=0.5$.

For $\alpha=0.5$ and the Hamiltonian of the harmonic oscillator the transformation matrix becomes equal to

$$
A=\left(\begin{array}{c:c:c:c}
-\frac{3}{5} g & -\frac{12 g}{5 \Delta t} & 1-g & -\omega^{2} \Delta t \frac{1-g}{4} \\
\frac{12 g}{5 \Delta t \omega^{2}} & -\frac{3}{5} g & \Delta t \frac{1-g}{4} & 1-g \\
1-g & -\omega^{2} \Delta t \frac{1-g}{4} & \frac{1}{15} g\left(\Delta t^{2} \omega^{2}-9\right) & \frac{4 g}{15 \Delta t}\left(\Delta t^{2} \omega^{2}-9\right) \\
\Delta t \frac{1-g}{4} & 1-g & -\frac{4 g}{15 \Delta t \omega^{2}}\left(\Delta t^{2} \omega^{2}-9\right) & \frac{1}{15} g\left(\Delta t^{2} \omega^{2}-9\right)
\end{array}\right)
$$

where $g=\frac{5 \Delta t^{2} \omega^{2}}{\Delta t^{2} \omega^{2}+16}$. The eigenvalues are computed numerically, see Fig. 3.8, which is a two-dimensional slice of the plot Fig. 3.7 with a fixed weight $\alpha=0.5$. Within the stability domain, all the eigenvalues are equal to one in modulus, which leads to an absence of dissipation in the numerical scheme. The numerical calculation of the eigenvalues provides the linear stability condition $|\omega \Delta t| \leq 1.757$, which is slightly more restrictive than the criteria for the Störmer-Verlet scheme. Compared with the scheme $P 2 N 3 Q 4 L o b$ in [113] we see that bumps are present in the linear stability domain for both cases, but the stability restriction for $P 2 N 3 Q 4 L o b$ is less restrictive and equals $|\omega \Delta t| \leq 2 \sqrt{2}$.

Since the value $\alpha=0.5$ gives the best stability results, the time integration scheme 3.35 becomes

$$
\begin{aligned}
q^{n+1 / 2} & =\frac{3}{4} q^{n,-}+\frac{1}{4} q^{n,+}+\frac{\Delta t}{4}\left(\frac{\partial H\left(p^{n,+}, q^{n,+}\right)}{\partial p^{n,+}}+\frac{\partial H\left(p^{n+1 / 2}, q^{n+1 / 2}\right)}{\partial p^{n+1 / 2}}\right) \\
p^{n+1 / 2} & =\frac{3}{4} p^{n,-}+\frac{1}{4} p^{n,+}-\frac{\Delta t}{4}\left(\frac{\partial H\left(p^{n,+}, q^{n,+}\right)}{\partial q^{n,+}}+\frac{\partial H\left(p^{n+1 / 2}, q^{n+1 / 2}\right)}{\partial q^{n+1 / 2}}\right) \\
q^{n+1,-} & =q^{n,+}+\Delta t \frac{\partial H\left(p^{n+1 / 2}, q^{n+1 / 2}\right)}{\partial p^{n+1 / 2}} \\
p^{n+1,-} & =p^{n,+}-\Delta t \frac{\partial H\left(p^{n+1 / 2}, q^{n+1 / 2}\right)}{\partial q^{n+1 / 2}} \\
q^{n+1,+} & =\frac{4}{3} q^{n+1 / 2}-\frac{1}{3} q^{n,+}+\frac{\Delta t}{3} \frac{\partial H\left(p^{n+1,-}, q^{n+1,-}\right)}{\partial p^{n+1,-}}
\end{aligned}
$$




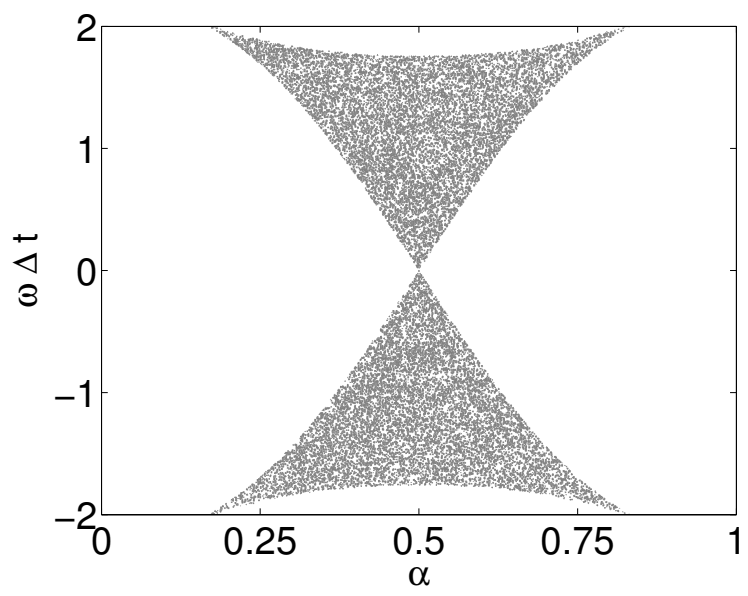

Figure 3.7: Modulus of eigenvalues $\lambda_{i}$ of system 3.35 for a linear harmonic oscillator. The shadowed area corresponds to the region where the modulus of all the eigenvalues is equal to one.

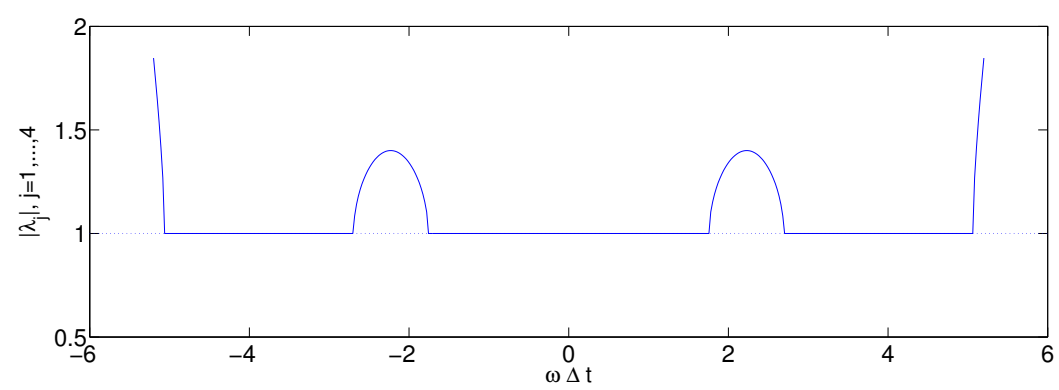

Figure 3.8: Modulus of the eigenvalues for the linear system 3.37 with $\alpha=0.5$ - solid line versus $\lambda=1-$ dotted line.

$$
p^{n+1,+}=\frac{4}{3} p^{n+1 / 2}-\frac{1}{3} p^{n,+}-\frac{\Delta t}{3} \frac{\partial H\left(p^{n+1,-}, q^{n+1,-}\right)}{\partial q^{n+1,-}} .
$$

The accuracy of the scheme (3.38) is verified for a linear system with Hamiltonian $H=\frac{1}{2} p^{2}+\frac{1}{2} \omega^{2} q^{2}$. The results in Table 3.1 show that the scheme 3.38) is third order accurate. We define $L^{2}$ error as $L_{e r r}^{2}=\sqrt{\sum_{n=1}^{N} \int_{t^{n-1}}^{t^{n}}\left(q^{\tau}-q_{e x}(t)\right)^{2} \mathrm{~d} t}$ for the polynomial approximation $q^{\tau}$ defined in $3.32 \mathrm{~b}$ and the exact solution of the autonomous system derived in 3.88 . The final result of the scheme are the values $q^{n+1,+}, p^{n+1,+}$, therefore 
$L^{\infty}$-error and $\Delta H$-error are defined as

$$
\begin{aligned}
& L_{e r r}^{\infty}=\max _{1 \leq n \leq N}\left|q^{n,+}-q_{e x}\left(t^{n}\right)\right|, \\
& \Delta H_{e r r}=\max _{1 \leq n \leq N}\left(H\left(p^{n,+}, q^{n,+}\right)\right)-\min _{1 \leq n \leq N}\left(H\left(p^{n,+}, q^{n,+}\right)\right) .
\end{aligned}
$$

\begin{tabular}{l|ll|ll|ll}
\hline \hline & \multicolumn{2}{|c|}{$q$} & \multicolumn{2}{c|}{$q$} & \multicolumn{2}{c}{ Energy } \\
\hline Step & $L^{\infty}$-error & order & $L^{2}$-error & order & $\Delta H$-error & order \\
\hline$\Delta t$ & $4.1204 \mathrm{E}-6$ & - & $6.1191 \mathrm{E}-6$ & - & $1.3489 \mathrm{E}-9$ & - \\
\hline$\Delta t / 2$ & $5.1937 \mathrm{E}-7$ & 2.9879 & $7.0457 \mathrm{E}-7$ & 3.1185 & $1.6565 \mathrm{E}-10$ & 3.0256 \\
\hline$\Delta t / 4$ & $6.5058 \mathrm{E}-8$ & 2.9970 & $8.6159 \mathrm{E}-8$ & 3.0317 & $2.0613 \mathrm{E}-11$ & 3.0065 \\
\hline$\Delta t / 8$ & $8.1366 \mathrm{E}-9$ & 2.9992 & $1.0710 \mathrm{E}-8$ & 3.0081 & $2.5735 \mathrm{E}-12$ & 3.0017 \\
\hline$\Delta t / 16$ & $1.0172 \mathrm{E}-9$ & 2.9998 & $1.3369 \mathrm{E}-9$ & 3.0020 & $3.2171 \mathrm{E}-13$ & 2.9999 \\
\hline$\Delta t / 32$ & $1.2716 \mathrm{E}-10$ & 3.0000 & $1.6705 \mathrm{E}-10$ & 3.0005 & $4.0211 \mathrm{E}-14$ & 3.0001 \\
\hline$\Delta t / 64$ & $1.5895 \mathrm{E}-11$ & 3.0000 & $2.0879 \mathrm{E}-11$ & 3.0001 & $5.0263 \mathrm{E}-15$ & 3.0000 \\
\hline
\end{tabular}

Table 3.1: Convergence in $L^{\infty}, L^{2}$-norms of the error in $q$ for a harmonic oscillator, and $L^{\infty}$ error in the energy of the system. Initial conditions are $q^{0,-}=q^{0,+}=-0.001$, $p^{0,-}=p^{0,+}=0, \omega^{2}=0.1$, the biggest time step $\Delta t=1$, the final time $T=40$. The precision set to solve the implicit system is $\epsilon=10^{-12}$.

\section{Dispersion relation}

The dispersion analysis is performed following [74]. In order to compute the dispersion properties of the new variational time integration scheme (3.38), we take the Fourier modes (i.e., we now take $\lambda=e^{-i \hat{\omega} \Delta t}$ such that $|\lambda|=1$ )

$$
\left(\begin{array}{l}
p^{n,-} \\
q^{n,-} \\
p^{n,+} \\
q^{n,+}
\end{array}\right)=\left(\begin{array}{l}
a e^{-i \hat{\omega} n \Delta t} \\
b e^{-i \hat{\omega} n \Delta t} \\
c e^{-i \hat{\omega} n \Delta t} \\
d e^{-i \hat{\omega} n \Delta t}
\end{array}\right)
$$

and substitute these into the system 3.37, which gives a linear system

$$
e^{-i \hat{\omega} \Delta t}(a, b, c, d)^{T}=A(a, b, c, d)^{T} .
$$

In general, we are not interested in the results for the variables $p^{n+1,-}, q^{n+1,-}$, as those are intermediate data. What matters is a relation for the variables $p^{n+1,+}, q^{n+1,+}$. In the continuum case, as shown in App.3.7.1 the exact dispersion relation is $c= \pm i d \omega$, (3.86). Nevertheless, as there are four equations, all of them have to be included in the analysis. We solve system of equations (3.41) with Matlab to find the relations between $a, b, c$ and $d$, and to obtain the numerical frequency $\hat{\omega}$. 
For the dispersion analysis we introduce the variables

$$
x=\sqrt{1-\frac{\Delta t^{6} \omega^{6}}{36\left(4-\Delta t^{2} \omega^{2}\right)^{2}}}, \quad y=\frac{\Delta t^{3} \omega^{3}}{6\left(4-\Delta t^{2} \omega^{2}\right)} .
$$

Notice, that $x \rightarrow 1, y \rightarrow 0$, when $\Delta t \rightarrow 0$. The first family of solutions of 3.41 is

$$
\begin{aligned}
& a=-i b \omega, \quad c=-i d \omega, \quad b=-(x+i y) d, \\
& \hat{\omega}=\frac{1}{\Delta t} \arccos \frac{-4\left(4-\Delta t^{2} \omega^{2}\right) x+\Delta t^{2} \omega^{2}\left(\frac{\Delta t^{2} \omega^{2}}{6}-3\right)}{\Delta t^{2} \omega^{2}+16} .
\end{aligned}
$$

We see, that the second relation in 3.43 is the exact one. The numerical frequency is, however, wrong as can be verified by taking the limit $\Delta t \rightarrow 0$ in $3.43 \mathrm{~b}$.

The second family of solutions is

$$
\begin{aligned}
& a=-i b \omega, \quad c=-i d \omega, \quad b=(x-i y) d, \\
& \hat{\omega}=\frac{1}{\Delta t} \arccos \frac{4\left(4-\Delta t^{2} \omega^{2}\right) x+\Delta t^{2} \omega^{2}\left(\frac{\Delta t^{2} \omega^{2}}{6}-3\right)}{\Delta t^{2} \omega^{2}+16} .
\end{aligned}
$$

The relation $c=-i d \omega$ is the exact dispersion relation, and the numerical frequency converges to the exact one $\hat{\omega} \rightarrow \omega$ if $\Delta t \rightarrow 0$ in 3.44b, see Fig. 3.9

The third family of solutions is

$$
\begin{aligned}
& a=i b \omega, \quad c=i d \omega, \quad b=(x+i y) d \\
& \hat{\omega}=-\frac{1}{\Delta t} \arccos \frac{4\left(4-\Delta t^{2} \omega^{2}\right) x+\Delta t^{2} \omega^{2}\left(\frac{\Delta t^{2} \omega^{2}}{6}-3\right)}{\Delta t^{2} \omega^{2}+16} .
\end{aligned}
$$

In this case the numerical frequency converges to the negative value, $\hat{\omega} \rightarrow-\omega$ when $\Delta t \rightarrow 0$.

The fourth family of solutions is

$$
\begin{aligned}
& a=i b \omega, \quad c=i d \omega, \quad b=(-x+i y) d \\
& \hat{\omega}=-\frac{1}{\Delta t} \arccos \frac{-4\left(4-\Delta t^{2} \omega^{2}\right) x+\Delta t^{2} \omega^{2}\left(\frac{\Delta t^{2} \omega^{2}}{6}-3\right)}{\Delta t^{2} \omega^{2}+16} .
\end{aligned}
$$

Here the numerical frequency does not converge to the correct value when $\Delta t \rightarrow 0$.

Since there are two parasitic modes we need to be careful that they do not pollute the solution. For the parasitic modes the relation $b \rightarrow-d$ holds. One important step is to choose $a=c$ and $b=d$, i.e. take $p^{0,-}=p^{0,+}$ and $q^{0,-}=q^{0,+}$ as initial conditions. In this case the parasitic modes are eliminated. For the nonlinear water wave case we verified this kind of filtering numerically (pending future analytical results), which appears to be sufficient to ensure stability in the nonlinear case. An absence of initial jump leads to satisfactory results, as will be demonstrated later in 4.5.1 


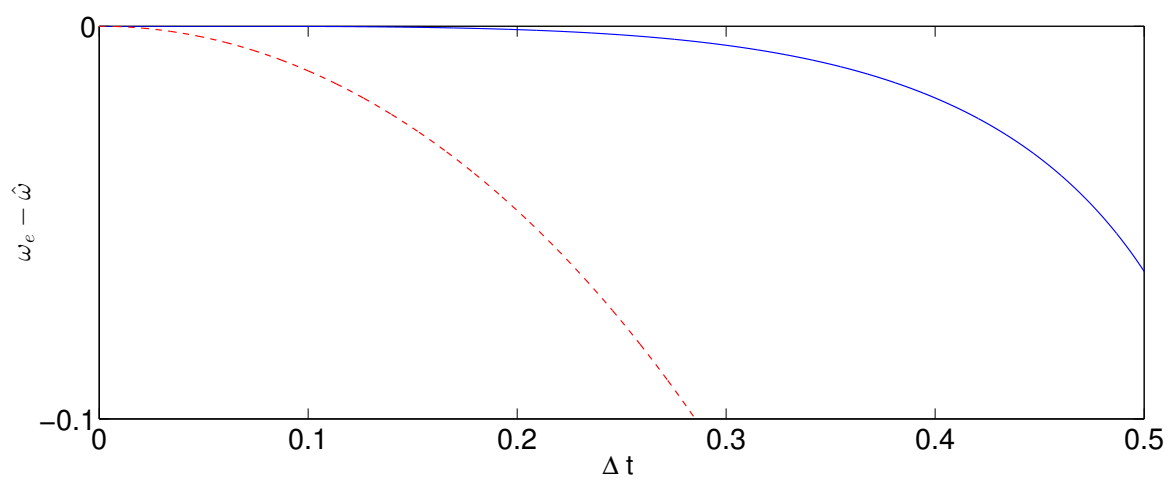

Figure 3.9: A diffirence between numerical and exact frequencies $\omega-\hat{\omega}$. In red dash we present the dispersion relation for the Störmer-Verlet scheme, in blue solid line the dispersion relation $3.44 \mathrm{~b}$ for the third order scheme 3.38 .

\section{Symplecticity}

If a one-degree-of-freedom Hamiltonian system is approximated with two variables $p^{n}$ and $q^{n}$, the symplecticity condition is formulated as

$$
M^{T} J M=J,
$$

where the Jacobian of the transformation is defined as

$$
M=\frac{\partial\left(p^{n+1}, q^{n+1}\right)}{\partial\left(p^{n}, q^{n}\right)} .
$$

The structure matrix $J$, defined as

$$
J=\left(\begin{array}{cc}
0 & I \\
-I & 0
\end{array}\right)
$$

where $I$ is the identity matrix with the dimension equal to the degrees of freedom of the Hamiltonian system, relates to the Hamiltonian system as

$$
\left(\begin{array}{l}
p \\
q
\end{array}\right)_{t}=J^{-1} \nabla H=\left(\begin{array}{cc}
0 & -1 \\
1 & 0
\end{array}\right)\left(\begin{array}{l}
H_{p} \\
H_{q}
\end{array}\right) .
$$

For the details we refer to [64]. Since we are working with an extended system, i.e. a onedegree-of-freedom Hamiltonian system is approximated with four variables $p^{n,-}, p^{n,+}$ and $q^{n,-}, q^{n,+}$, the matrices in the symplecticity condition 3.47 are also extended. We take

$$
M=\frac{\partial\left(p^{n+1,-}, p^{n+1,+}, q^{n+1,-}, q^{n+1,+}\right)}{\partial\left(p^{n,-}, p^{n,+}, q^{n,-}, q^{n,+}\right)},
$$


and the structure matrix $J$, defined as in 3.49 for a two-dimensional system, relates to the Hamiltonian system as

$$
\left(\begin{array}{l}
p^{-} \\
p^{+} \\
q^{-} \\
q^{+}
\end{array}\right)_{t}=(J)^{-1} \nabla H=\left(\begin{array}{cccc}
0 & 0 & 0 & -1 \\
0 & 0 & -1 & 0 \\
0 & 1 & 0 & 0 \\
1 & 0 & 0 & 0
\end{array}\right)\left(\begin{array}{l}
H_{p^{-}} \\
H_{p^{+}} \\
H_{q^{-}} \\
H_{q^{+}}
\end{array}\right)
$$

We verified in Matlab that the condition (3.47) is valid for the new system (3.38). The detail of the matrix $M$ are not given explicitly here since the expressions are too large to present.

For a Hamiltonian system with one degree of freedom the symplecticity means the preservation of an area in phase space, [64]. A phase-space plane is shown on Figure 3.10 A point on the plane is given as $\left(p^{n,+}, q^{n,+}\right)$, as our prime interest lies in these variables. We define four initial conditions in the plane, that constitute a quadrilateral shape. The numerical approximation of a harmonic oscillator system develops according to scheme (3.38). The system cycles over time through the same states which are defined by the initial conditions. Area of the rectangular shape oscillates around the initial state, see Fig 3.11

Nevertheless, from a geometrical point of view, the symplecticity condition (3.47) means in the general case with $d>1$ degrees of freedom that the sum of the oriented areas of the projections of a shape in $2 d$ space onto the planes $\left(p^{i}, q^{i}\right)$ is conserved [64]. Therefore Figures 3.10 and 3.11 do not prove symplecticity, but illustrate the property of the plus-variables $p^{n,+}, q^{n,+}$ to cycle through the same states. Symplecticty of the discrete map is verified with condition (3.47).

The conclusions on the novel third order scheme (3.38) are as follows. The scheme is symplectic, but the linear stability condition is more restrictive than the condition for the Störmer-Verlet scheme. The scheme is third order accurate, which is verified both for linear and nonlinear problems (an application to nonlinear water waves is presented in Sec. 4.5.1.

\subsection{Applications to non-autonomous systems}

In the following section we describe issues regarding the time integration of nonautonomous Hamiltonian systems. 


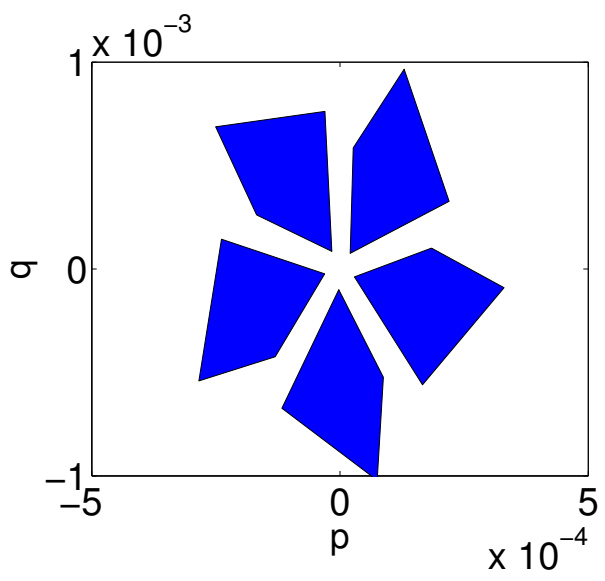

Figure 3.10: Evolution of variables $\left(p^{n,+}, q^{n,+}\right)$ in phase space over one time period. System (3.38) with $\omega=0.3162, \Delta t=0.2484$ is solved for four initial conditions. The error in solving the linear system in the implicit step of 3.38 is set to $\epsilon=10^{-15}$.

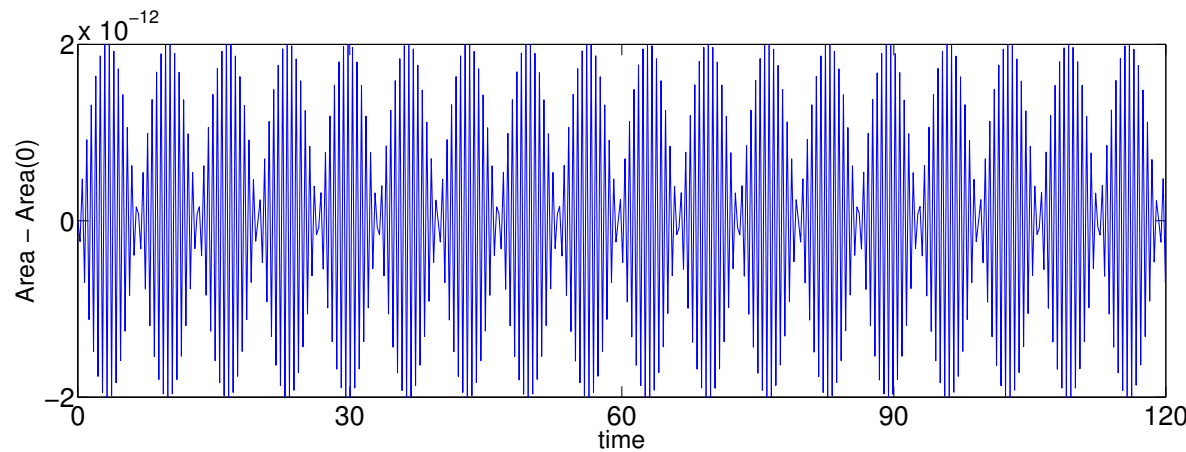

Figure 3.11: Oscillation in the area of a quadrilateral shape in phase-space shown in Fig 3.10 for the harmonic oscillator computed with the third order accurate symplectic time integrator 3.38). System 3.38 is solved over 10 time periods with $\omega=0.3162$, $\Delta t=0.2484$. The precision in solving the implicit step in 3.38 is set to $\epsilon=10^{-15}$. 


\subsubsection{Damped oscillator ${ }^{6}$}

Consider a damped one-degree-of-freedom Hamiltonian system with the following non-autonomous variational principle, [115]:

$$
\begin{aligned}
0 & =\delta \tilde{\mathcal{L}}(p, q, t)=\delta \int_{0}^{T}\left(p \frac{\mathrm{d} q}{\mathrm{~d} t}-H(p, q)\right) e^{\gamma t} \mathrm{~d} t \\
& =\int_{0}^{T}\left(\frac{\mathrm{d} q}{\mathrm{~d} t}-\frac{\partial H}{\partial p}\right) \delta\left(p e^{\gamma t}\right)-\left(\frac{\mathrm{d}\left(p e^{\gamma t}\right)}{\mathrm{d} t}+\frac{\partial H}{\partial q}\right) e^{\gamma t} \delta q \mathrm{~d} t+\left.p e^{\gamma t} \delta q\right|_{0} ^{T},
\end{aligned}
$$

with $\gamma>0$ the coefficient of the linear momentum damping and end-point conditions $\delta q(0)=\delta q(T)=0$. Variation of (3.53) with respect to the variables $p$ and $q$ yields Hamilton's equations for the damped oscillator when the Hamiltonian $H(p, q)=\frac{1}{2} p^{2}+$ $\frac{1}{2} q^{2}$ (and unit frequency). These equations are given by

$$
\begin{aligned}
& \delta\left(p e^{\gamma t}\right): \quad \frac{\mathrm{d} q}{\mathrm{~d} t}=\frac{\partial H}{\partial p}=p, \\
& \delta q: \quad \frac{\mathrm{d} p}{\mathrm{~d} t}+\gamma p=-\frac{\partial H}{\partial q}=-q .
\end{aligned}
$$

We perform the coordinate transformation $q=Q \exp (-\gamma t / 2)$ and $p=P \exp (-\gamma t / 2)]^{7}$ The variational principle then becomes

$$
0=\delta \int_{0}^{T}\left(P \frac{\mathrm{d} Q}{\mathrm{~d} t}-\tilde{H}(P, Q)\right) \mathrm{d} t
$$

with modified Hamiltonian

$$
\tilde{H}(P, Q)=\frac{1}{2} P^{2}+\frac{1}{2} Q^{2}+\frac{1}{2} \gamma P Q .
$$

Hence, the system 3.55) is reformulated as an autonomous system, viz. the modified Hamiltonian (3.56) is time independent in the new variables $P$ and $Q$. As this is a property of the continuous system, we want to monitor it at the discrete level.

\footnotetext{
${ }^{6}$ The essence of this case has been used in journal article [141] for linearly damped water waves.

${ }^{7}$ The analytical part of this section was first investigated by $\mathrm{O}$. Bokhove.
} 


\section{Discretization}

We start with a first order approximation of the functional 3.53 in time. We consider the discrete variational principle

$$
\tilde{\mathcal{L}}^{\tau}\left(p^{\tau}, q^{\tau}, t\right):=\sum_{n=0}^{N-1} \int_{t^{n}}^{t^{n+1}}\left(p^{\tau} \frac{\mathrm{d} q^{\tau}}{\mathrm{d} t}-H\left(p^{\tau}, q^{\tau}\right)\right) e^{\gamma t} \mathrm{~d} t-\left.\sum_{n=-1}^{N-1}\left[\left[q^{\tau}\right]\right]\left\{\left\{p^{\tau} e^{\gamma t}\right\}\right\}_{\alpha}^{\beta}\right|_{t_{n+1}},
$$

where the variables $(p, q)$ are approximated as piecewise-constant per time interval $\left(t^{n}, t^{n+1}\right)$. As we have discussed in Section 3.3.2 to obtain the well-known formulation of the symplectic Euler scheme, we need to rename the approximations as

$$
q^{\tau}=q^{n,+}, \quad p^{\tau}=p^{n+1,-} .
$$

For the average term in the flux we have chosen $\alpha=1, \beta=0$ as it was demonstrated in Section 3.3.2 to result in one of the forms of the symplectic Euler scheme. The difference with the case considered in Section 3.3.2 is the exponential term responsible for damping. It will be approximated using the value at the right boundary of the time element $e^{\gamma t^{n+1,-}}$. An additional comment has to be made here. Since we are working with piecewise continuous approximations and time is also a variable here, a uniform approximation of a variable has to be taken within the element $\left(t^{n}, t^{n+1}\right)$. Therefore, if the left trace at time $t^{n+1}$ is called $t^{n+1,-}$, we take the approximation in the time interval $\left(t^{n}, t^{n+1}\right)$ to be $t^{n+1,-}$. One has to keep in mind, that here $t^{n+1,-} \neq t^{n+1,+}$ as it is an element wise constant approximation. Substituting the approximations 3.58 into the discrete functional (3.57) we get

$$
\begin{aligned}
\tilde{\mathcal{L}}^{\tau}\left(p^{\tau}, q^{\tau}, t\right) & :=\sum_{n=0}^{N-1}-\Delta t H\left(p^{n+1,-}, q^{n,+}\right) e^{\gamma t^{n+1,-}} \\
& -\sum_{n=-1}^{N-1}\left(q^{n,+}-q^{n+1,+}\right)\left(p^{n+1,-} e^{\gamma t^{n+1,-}}\right) .
\end{aligned}
$$

To obtain the dynamics we compute the discrete variational principle $\delta \tilde{\mathcal{L}}^{\tau}=0$. We take variations of 3.59 with respect to the variables $p^{n+1,-}, q^{n,+}$, use an arbitrariness of variations and endpoint conditions $\delta q^{0,-}=0, \delta p^{0,-}=0, \delta q^{N,+}=0$, which results in

$$
\begin{gathered}
\delta q^{n,+}: \quad e^{\gamma t^{n+1,-}} p^{n+1,-}=e^{\gamma t^{n,-}} p^{n,-}-\Delta t e^{\gamma t^{n+1,-}} \frac{\partial H\left(p^{n+1,-}, q^{n,+}\right)}{\partial q^{n,+}}, \\
\delta p^{n+1,-}: \quad e^{\gamma t^{n+1,-}} q^{n+1,+}=e^{\gamma t^{n+1,-}} q^{n,+}+\Delta t e^{\gamma t^{n+1,-}} \frac{\partial H\left(p^{n+1,-}, q^{n,+}\right)}{\partial p^{n+1,-}} .
\end{gathered}
$$


After division by the exponential term $e^{\gamma t^{n+1,-}}$, we arrive at

$$
\begin{aligned}
& p^{n+1,-}=p^{n,-}-\Delta t \frac{\partial H\left(p^{n+1,-}, q^{n,+}\right)}{\partial q^{n,+}}-\left(1-e^{\gamma\left(t^{n,-}-t^{n+1,-}\right)}\right) p^{n,-}, \\
& q^{n+1,+}=q^{n,+}+\Delta t \frac{\partial H\left(p^{n+1,-}, q^{n,+}\right)}{\partial p^{n+1,-}}
\end{aligned}
$$

In this time integration scheme the first step is implicit and the second step is explicit, which corresponds to the symplectic Euler scheme.

The exponential term in (3.61a) needs some clarification. We can approximate

$$
1-e^{\gamma\left(t^{n,-}-t^{n+1,-}\right)}=1-e^{-\gamma \Delta t} \approx \gamma \Delta t
$$

which shows that the discrete equation $3.61 \mathrm{a}$ is an approximation to the damped equation

$$
\dot{p}+H_{q}+\gamma p=0 .
$$

This approximation ensures that the modified total energy 3.56 will be preserved as will be demonstrated next.

\section{Numerical results}

Consider the Hamiltonian $H(p, q)=\frac{1}{2} p^{2}+\frac{1}{2} q^{2}$. We compare three cases for the damped variational principle, viz. approximation 3.61a and the more straightforward approximations for the damping term.

$$
p^{n+1}=p^{n}-\Delta t \frac{\partial H\left(p^{n+1}, q^{n}\right)}{\partial q^{n}}-\Delta t \gamma p^{n},
$$

or

$$
p^{n+1}=p^{n}-\Delta t \frac{\partial H\left(p^{n+1}, q^{n}\right)}{\partial q^{n}}-\Delta t \gamma p^{n+1},
$$

while the second equation $3.61 \mathrm{~b}$ in the system remains unchanged. We verify the absence of drift in a discrete version of the modified energy 3.56 at each time level $t_{n}$, $n=0, \ldots, N$,

$$
H_{e x}\left(p^{n}, q^{n}, t^{n}\right)=\frac{1}{2}\left(\left(p^{n}\right)^{2}+\left(q^{n}\right)^{2}+\gamma p^{n} q^{n}\right) e^{\gamma t^{n}}
$$

for the different approximations. The results of the discrete modified energy are presented in Fig. 3.12 for the different approximations versus time. We see, that the modified energy for approximation 3.61a oscillates around the initial state, while the other two approximations reveal a drift in energy. The latter was expected for the backward approximation of the forcing term in (3.64). Nevertheless, the result is unexpected for implicit scheme (3.65), where the approximation of the damping term is done analogously to the symplectic Euler scheme. Therefore our variational approach allows also the derivation of time integrators that result in bounded fluctuation of special integrals. 


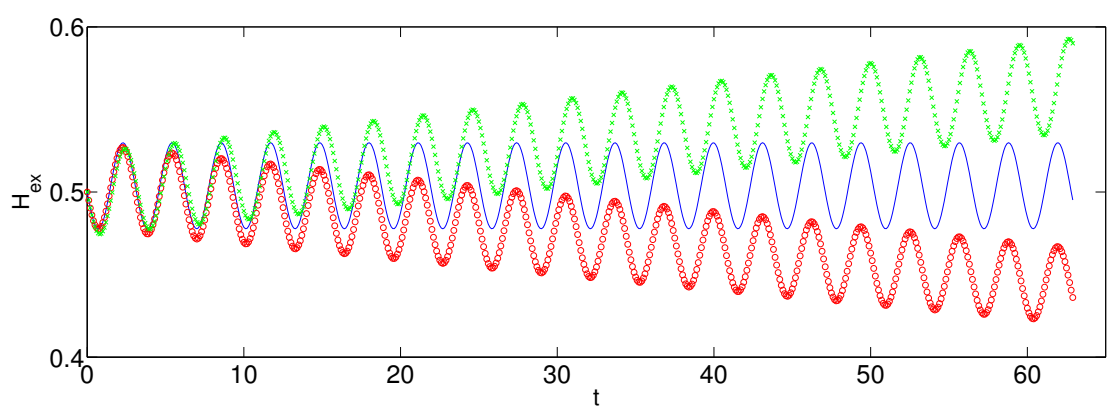

Figure 3.12: Modified total energy $H_{e x}$ for a damped oscillator versus time. The discrete modified energy for approximation 3.61a is plotted as a solid line, the discrete modified energy for approximation 3.65 is plotted with crosses and the discrete modified energy for approximation 3.64 is plotted versus time with circles.

\subsubsection{Forced oscillator}

In this section we study a forced Hamiltonan system. The Lagrangian 3.1 with added forcing is formulated as

$$
\mathcal{L}(p, q, t):=\int_{0}^{T}\left(p \frac{\mathrm{d} q}{\mathrm{~d} t}-H(p, q)-f(p, q, t)\right) \mathrm{d} t,
$$

We consider the simple case of a forced oscillator, where the point of suspension is moving in the horizontal direction. The Hamiltonian is $H(p, q)=\frac{1}{2} p^{2}+\frac{1}{2} q^{2}$ and the forcing function $f(p, q, t)=-a \gamma t q$, where $a$ is the amplitude and $\gamma$ the frequency of the forcing. The corresponding system of equation is

$$
\frac{\mathrm{d} q}{\mathrm{~d} t}=p \quad \text { and } \quad \frac{\mathrm{d} p}{\mathrm{~d} t}=-q+a \gamma t .
$$

This system is non-autonomous, but we can introduce a coordinate transformation to make it autonomous, which can be computed using the coordinate transformation

$$
q=Q+a \gamma t, \quad p=P .
$$

The Lagrangian 3.67 for a forced harmonic oscillator is formulated in the new variables as

$$
\mathcal{L}(p, q, t)=\int_{0}^{T}\left(P \frac{\mathrm{d} Q}{\mathrm{~d} t}-\left(\frac{1}{2} P^{2}+\frac{1}{2} Q^{2}-a \gamma P\right)\right) \mathrm{d} t+\frac{1}{6} a^{2} \gamma^{2} T^{3}
$$


An important quantity to monitor is the Hamiltonian in the new coordinates

$$
\hat{H}=\frac{1}{2} P^{2}+\frac{1}{2} Q^{2}-a \gamma P
$$

which can also be used to compute the order of accuracy of the forced scheme. The exact solution to the problem 3.68 is

$$
q_{e x}=q(0) \cos (t)+(p(0)-a \gamma) \sin (t)+a \gamma t
$$

Next, we will consider the symplectic Störmer-Verlet scheme to compute the evolution of the forced system.

\section{Forced symplectic Störmer-Verlet scheme}

We consider the second order approximation with added forcing, and investigate the accuracy for different choices of the forcing term 8 . We construct a Störmer-Verlet time stepping method that is adjoint to the one discussed in detail in Chapter 3.3.3. We take appropriate polynomial approximations (3.21), mixed quadrature rule (3.22) and $\alpha=0$. Then we obtain a discrete approximation of the Lagrangian (3.67) formulated as follows

$$
\begin{aligned}
\mathcal{L}^{\tau}\left(p^{\tau}, q^{\tau}, t\right)= & \sum_{n=0}^{N-1}\left(p^{n+1 / 2}\left(q^{n+1,-}-q^{n,+}\right)\right. \\
& \left.-\frac{\Delta t}{2}\left(H\left(p^{n+1 / 2}, q^{n,+}\right)+H\left(p^{n+1 / 2}, q^{n+1,-}\right)\right)\right) \\
& \left.-\frac{\Delta t}{2}\left(f\left(p^{n+1 / 2}, q^{n,+}, t^{*}\right)+f\left(p^{n+1 / 2}, q^{n+1,-}, t^{*}\right)\right)\right) \\
& -\sum_{n=-1}^{N-1}\left(q^{n+1,-}-q^{n+1,+}\right) p^{n+1,+} .
\end{aligned}
$$

\footnotetext{
${ }^{8}$ Investigation of a general first order forced variational principle within the current approach was first done by V. Ambati and O. Bokhove.
} 
Taking the variations, we obtain the following system of equations

$$
\begin{aligned}
\delta p^{n,+}: & q^{n+1,+}=q^{n+1,-}, \\
\delta q^{n,+}: & p^{n+1 / 2}=p^{n,+}-\frac{\Delta t}{2} \frac{\partial H\left(p^{n+1 / 2}, q^{n,+}\right)}{\partial q^{n,+}}-\frac{\Delta t}{2} \frac{\partial f\left(p^{n+1 / 2}, q^{n,+}, t^{*}\right)}{\partial q^{n,+}} \\
\delta p^{n+1 / 2}: & q^{n+1,-}=q^{n,+}+\frac{\Delta t}{2}\left(\frac{\partial H\left(p^{n+1 / 2}, q^{n,+}\right)}{\partial p^{n+1 / 2}}+\frac{\partial H\left(p^{n+1 / 2}, q^{n+1,-}\right)}{\partial p^{n+1 / 2}}\right) \\
& +\frac{\Delta t}{2}\left(\frac{\partial f\left(p^{n+1 / 2}, q^{n++}, t^{*}\right)}{\partial p^{n+1 / 2}}+\frac{\partial f\left(p^{n+1 / 2}, q^{n+1,-}, t^{*}\right)}{\partial p^{n+1 / 2}}\right) \\
\delta q^{n+1,-}: & p^{n+1,+}=p^{n+1 / 2}-\frac{\Delta t}{2} \frac{\partial H\left(p^{n+1 / 2}, q^{n+1,-}\right)}{\partial q^{n+1,-}}-\frac{\Delta t}{2} \frac{\partial f\left(p^{n+1 / 2}, q^{n+1,-}, t^{*}\right)}{\partial q^{n+1,-}}
\end{aligned}
$$

where we decide on the choice of time level $t^{*}$ later. Convergence tests are performed to compare different forcing term approximations. We use the following approximations of the integral on the forcing term in 3.74, viz.

$$
\begin{aligned}
& \int_{t_{n}}^{t_{n+1}} f\left(p^{\tau}, q^{\tau}\right) \mathrm{d} t \\
& \quad=\frac{\Delta t}{2}\left(f\left(p^{n+1 / 2}, q^{n,+}, t^{n}\right)+f\left(p^{n+1 / 2}, q^{n+1,-}, t^{n+1}\right)\right),
\end{aligned}
$$

or

$$
\begin{aligned}
& \int_{t_{n}}^{t_{n+1}} f\left(p^{\tau}, q^{\tau}, t\right) \mathrm{d} t \\
& \quad=\frac{\Delta t}{2}\left(f\left(p^{n+1 / 2}, q^{n,+}, t^{n+1 / 2}\right)+f\left(p^{n+1 / 2}, q^{n+1,-}, t^{n+1 / 2}\right)\right) .
\end{aligned}
$$

The convergence tests are based on the $L^{\infty}$-norm, see Table 3.2 , and the $L^{2}$-norm, see Table 3.3. The estimation of the extended energy error 3.71 is given in Table 3.4. The exact solution (3.72), here called $q_{e x}$, is used.

The set up of the numerical test is as follows. We start with $\Delta t=1$, the number of time steps is $N=40$. The final time, where we compare the results, is $T=40$. The amplitude of the forcing is $a=0.1$ and the frequency of the forcing is $\gamma=0.1$. The initial coordinate $q_{0}=0.1$ and the initial momentum $p_{0}=-0.1$. The errors are defined 
as

$$
\begin{aligned}
& L_{e r r}^{\infty}=\max _{1 \leq n \leq N}\left|q^{n}-q_{e x}\left(t^{n}\right)\right|, \\
& L_{e r r}^{2}=\sqrt{\sum_{n=1}^{N} \int_{t^{n-1}}^{t^{n}}\left(q^{\tau}-q_{e x}(t)\right)^{2} \mathrm{~d} t, \text { and }} \\
& \Delta \hat{H}_{e r r}=\max _{1 \leq n \leq N}\left(\hat{H}\left(p^{n}, q^{n}, t^{n}\right)\right)-\min _{1 \leq n \leq N}\left(\hat{H}\left(p^{n}, q^{n}, t^{n}\right)\right),
\end{aligned}
$$

where $q^{\tau}$ is an approximation of $q$ in the time interval $\left(t^{n-1}, t^{n}\right)$. For the case considered here, viz. a second order approximation, we have $q^{\tau}$ defined in 3.21.

\begin{tabular}{ll|ll|ll}
\hline \hline & & \multicolumn{2}{|c|}{ SV1 } & & \multicolumn{2}{c}{ SV2 } & \\
\hline Step & & $L_{\text {err }}^{\infty}$ & order & $L_{\text {err }}^{\infty}$ & order \\
\hline$\Delta t$ & $q$ & $2.2291 \mathrm{E}-1$ & - & $2.2041 \mathrm{E}-1$ & - \\
\hline$\Delta t / 2$ & $q$ & $6.2108 \mathrm{E}-2$ & 1.8436 & $6.1503 \mathrm{E}-2$ & 1.8414 \\
\hline$\Delta t / 4$ & $q$ & $1.5590 \mathrm{E}-2$ & 1.9942 & $1.5466 \mathrm{E}-2$ & 1.9915 \\
\hline$\Delta t / 8$ & $q$ & $3.8804 \mathrm{E}-3$ & 2.0063 & $3.8516 \mathrm{E}-3$ & 2.0056 \\
\hline$\Delta t / 16$ & $q$ & $9.6874 \mathrm{E}-4$ & 2.0020 & $9.6168 \mathrm{E}-4$ & 2.0018 \\
\hline$\Delta t / 32$ & $q$ & $2.4210 \mathrm{E}-4$ & 2.0005 & $2.4034 \mathrm{E}-4$ & 2.0005 \\
\hline$\Delta t / 64$ & $q$ & $6.0518 \mathrm{E}-5$ & 2.0001 & $6.0080 \mathrm{E}-5$ & 2.0001 \\
\hline
\end{tabular}

Table 3.2: Convergence in the $L^{\infty}$-norm of the error in $q$ for a forced harmonic oscillator. We denote (3.74) with the forcing term integral approximated as (3.75) by $S V 1$, and (3.74) with the forcing term integral approximated as 3.76 by $S V 2$.

\begin{tabular}{ll|ll|ll}
\hline \hline & & \multicolumn{2}{|c|}{ SV1 } & & \multicolumn{2}{c}{ SV2 } & \\
\hline Step & & $L_{e r r}^{2}$ & order & $L_{\text {err }}^{2}$ & order \\
\hline$\Delta t$ & $q$ & $6.8361 \mathrm{E}-1$ & - & $6.7380 \mathrm{E}-1$ & - \\
\hline$\Delta t / 2$ & $q$ & $1.7151 \mathrm{E}-1$ & 1.9949 & $1.6961 \mathrm{E}-1$ & 1.9901 \\
\hline$\Delta t / 4$ & $q$ & $4.2441 \mathrm{E}-2$ & 2.0147 & $4.2012 \mathrm{E}-2$ & 2.0134 \\
\hline$\Delta t / 8$ & $q$ & $1.0576 \mathrm{E}-2$ & 2.0047 & $1.0471 \mathrm{E}-2$ & 2.0043 \\
\hline$\Delta t / 16$ & $q$ & $2.6417 \mathrm{E}-3$ & 2.0012 & $2.6158 \mathrm{E}-3$ & 2.0011 \\
\hline$\Delta t / 32$ & $q$ & $6.6029 \mathrm{E}-4$ & 2.0003 & $6.5381 \mathrm{E}-3$ & 2.0003 \\
\hline$\Delta t / 64$ & $q$ & $1.6506 \mathrm{E}-4$ & 2.0001 & $1.6344 \mathrm{E}-3$ & 2.0001 \\
\hline
\end{tabular}

Table 3.3: Convergence in the $L^{2}$-norm of the error in $q$ for a forced harmonic oscillator. For details, see the caption of Table 3.2

These schemes are all second order and the extended energy $\hat{H}$ is bounded and reveals no drift in time. 


\subsection{Conclusions}

We have constructed novel symplectic time integrators using a variational approach. Also, we demonstrated how several well-known time integrators, such as the symplectic Euler, Störmer-Verlet, and symplectic modified midpoint time integrators, can be derived using this variational approach. The time integration method is based on a discontinuous Galerkin discretization in time, using a novel numerical flux, which connects the different time slabs. The new symplectic time integrators are second and third order accurate. The third order time integrator has excellent dispersion properties and also successfully tested on nonlinear water waves in a basin, as will be demonstrated in Chapter 4.5.1. The variational approach to derive time stepping schemes was also tested for non-autonomous Hamiltonian systems such as forced and damped oscillators.

\subsection{Appendix}

\subsubsection{Continuum harmonic oscillator}

In this section we will show the essential steps in the analysis of the harmonic oscillator following work in [7]. The dynamics of a harmonic oscillator is embedded in the functional

$$
\mathcal{L}(p, q):=\int_{0}^{T}\left(p \frac{\mathrm{d} q}{\mathrm{~d} t}-H(p, q)\right) \mathrm{d} t
$$

with $p(t)$ the momentum, $q(t)$ the coordinate and $H(p, q)$ the energy (or Hamiltonian) of the oscillator, which is defined as

$$
H(p, q):=\frac{1}{2} p^{2}+\frac{1}{2} \omega^{2} q^{2}
$$

\begin{tabular}{ll|ll|ll}
\hline \hline & \multicolumn{2}{|c|}{ SV1 } & \multicolumn{2}{c}{ SV2 } & \\
\hline Step & & $\Delta \hat{H}_{\text {err }}$ & order & $\Delta \hat{H}_{\text {err }}$ & order \\
\hline$\Delta t$ & $q$ & $2.7500 \mathrm{E}-3$ & - & $3.0625 \mathrm{E}-3$ & - \\
\hline$\Delta t / 2$ & $q$ & $7.1553 \mathrm{E}-4$ & 1.9423 & $8.0497 \mathrm{E}-4$ & 1.9277 \\
\hline$\Delta t / 4$ & $q$ & $1.7416 \mathrm{E}-4$ & 2.0386 & $1.9731 \mathrm{E}-4$ & 2.0285 \\
\hline$\Delta t / 8$ & $q$ & $4.3256 \mathrm{E}-5$ & 2.0094 & $4.9234 \mathrm{E}-5$ & 2.0027 \\
\hline$\Delta t / 16$ & $q$ & $1.0797 \mathrm{E}-5$ & 2.0023 & $1.2296 \mathrm{E}-5$ & 2.0015 \\
\hline$\Delta t / 32$ & $q$ & $2.6981 \mathrm{E}-6$ & 2.0006 & $3.0731 \mathrm{E}-6$ & 2.0004 \\
\hline$\Delta t / 64$ & $q$ & $6.7446 \mathrm{E}-7$ & 2.0001 & $7.6824 \mathrm{E}-7$ & 2.0001 \\
\hline
\end{tabular}

Table 3.4: Convergence of the extended energy error 3.77c for a forced harmonic oscillator. For details, see the caption of Table 3.2 . 
The variational derivative of the Hamiltonian is equal to

$$
\delta H(p, q):=\frac{\delta H}{\delta p} \delta p+\frac{\delta H}{\delta q} \delta q=p \delta p+\omega^{2} q \delta q .
$$

Next, we apply the variational principle

$$
\delta \mathcal{L}(p, q)=\int_{0}^{T}\left(\frac{\mathrm{d} q}{\mathrm{~d} t} \delta p+p \frac{\mathrm{d} \delta q}{\mathrm{~d} t}-\frac{\delta H}{\delta p} \delta p-\frac{\delta H}{\delta q} \delta q\right) \mathrm{d} t=0 .
$$

With the endpoint conditions $\Delta q(0)=0, \Delta q(T)=0$, the equations describing the dynamics of the harmonic oscillator emerge as follows:

$$
\frac{\mathrm{d} q}{\mathrm{~d} t}-\frac{\delta H}{\delta p}=0 \quad \text { and } \quad \frac{\mathrm{d} p}{\mathrm{~d} t}+\frac{\delta H}{\delta q}=0,
$$

with initial conditions $p(t=0)=p_{0}$ and $q(t=0)=q_{0}$. The system can be represented in matrix form as

$$
\left(\begin{array}{l}
p \\
q
\end{array}\right)_{t}=\left(\begin{array}{cc}
0 & -\omega^{2} \\
1 & 0
\end{array}\right)\left(\begin{array}{l}
p \\
q
\end{array}\right)=A\left(\begin{array}{l}
p \\
q
\end{array}\right)
$$

where a subscript $t$ refers to differentiation in time. The characteristic polynomial for the system matrix $A$ is

$$
\lambda^{2}+\omega^{2}=0
$$

therefore the eigenvalues are $\lambda= \pm i \omega$.

Following, for example, [74], we can find the dispersion relation by taking a Fourier mode

$$
\left(\begin{array}{l}
p \\
q
\end{array}\right)=\left(\begin{array}{l}
a e^{-i \hat{\omega} t} \\
b e^{-i \hat{\omega} t}
\end{array}\right)
$$

Solving it we arrive to

$$
\hat{\omega}=\omega, \quad a=-i \omega b
$$

or

$$
\hat{\omega}=-\omega, \quad a=i \omega b .
$$

Also we can write the exact solution of this system as

$$
\left(\begin{array}{l}
p \\
q
\end{array}\right)=\left(\begin{array}{cc}
\cos (\omega t) & -\omega \sin (\omega t) \\
\frac{1}{\omega} \sin (\omega t) & \cos (\omega t)
\end{array}\right)\left(\begin{array}{l}
p_{0} \\
q_{0}
\end{array}\right) .
$$




\subsubsection{Störmer-Verlet scheme}

In this section we provide the details of the analysis of scheme 3.20 introduced in Section 3.3.3. We demonstrate, that the scheme coincides with the Störmer-Verlet time stepping scheme.

In the system of equations 3.20 we have three variables from the time slab $\left(t^{n-1}, t^{n}\right)$ : $q^{n-1,+}, q^{n-1 / 2}, p^{n,-}$; four variables from the time slab $\left(t^{n}, t^{n+1}\right): p^{n,+}, q^{n,+}, q^{n+1 / 2}$, $p^{n+1,-}$, and two variables from the time slab $\left(t^{n+1}, t^{n+2}\right): p^{n+1,+}, q^{n+1,+}$. One can notice, that variables $q^{n-1 / 2}, q^{n-1,+}$ appear only in combination. To make the system solvable, we reformulate 3.20 with the variables $\left.[[q]]\right|_{t_{n+1}}, q^{n+1 / 2}$ and $\left.[[p]]\right|_{t_{n+1}}, p^{n+1,-}$ as unknowns:

$$
\begin{aligned}
\delta q^{n,+}: & \left.\beta[[p]]\right|_{t_{n+1}}=\left.\alpha[[p]]\right|_{t_{n}}, \\
\delta p^{n,+}: & q^{n+1 / 2}=q^{n,+}+\left.\beta[[q]]\right|_{t_{n}}+\frac{\Delta t}{2} \frac{\partial H\left(p^{n,+}, q^{n+1 / 2}\right)}{\partial p^{n,+}}, \\
\delta q^{n+1 / 2}: & p^{n+1,-}=p^{n,+}+\left.2 \beta[[p]]\right|_{t_{n+1}} \\
& -\frac{\Delta t}{2}\left(\frac{\partial H\left(p^{n,+}, q^{n+1 / 2}\right)}{\partial q^{n+1 / 2}}+\frac{\partial H\left(p^{n+1,-}, q^{n+1 / 2}\right)}{\partial q^{n+1 / 2}}\right), \\
\delta p^{n+1,-}: & \left.\alpha[[q]]\right|_{t_{n+1}}=q^{n+1 / 2}-q^{n,+}-\frac{\Delta t}{2} \frac{\partial H\left(p^{n+1,-}, q^{n+1 / 2}\right)}{\partial p^{n+1,-}} .
\end{aligned}
$$

In addition, we have the relations: $q^{n+1,+}=q^{n+1,-}-\left.[[q]]\right|_{t_{n+1}}=2 q^{n+1 / 2}-q^{n,+}-$ $\left.[[q]]\right|_{t_{n+1}}$, and $p^{n+1,+}=p^{n+1,-}-\left.[[p]]\right|_{t_{n+1}} ;$ therefore there are enough equations to close the system. 


\section{Linear stability}

To study the linear stability of the system of equations 3.89 we take $H=\frac{1}{2} p^{2}+$ $\frac{1}{2} \omega^{2} q^{2}$. Equations 3.89 for this Hamiltonian are written in matrix form as

$$
\begin{aligned}
& \left(\begin{array}{c}
{\left.[[p]]\right|_{t^{n+1}}} \\
p^{n+1,+} \\
{\left.[[q]]\right|_{t^{n+1}}} \\
q^{n+1,+}
\end{array}\right)=A^{n}\left(\begin{array}{c}
{\left.[[p]]\right|_{t^{n}}} \\
p^{n,+} \\
{\left.[[q]]\right|_{t^{n}}} \\
q^{n,+}
\end{array}\right) \\
& =\left(\begin{array}{c:c:c:c}
\frac{\alpha}{\beta} & 0 & 0 & 0 \\
2 \alpha-\frac{\alpha}{\beta} & 1-\frac{\Delta t^{2} \omega^{2}}{2} & -\Delta t \omega^{2} \beta & -\Delta t \omega^{2} \\
-\Delta t & \frac{\Delta t^{3} \omega^{2}}{4 \alpha} & \frac{\beta}{\alpha}\left(1+\frac{\Delta t^{2} \omega^{2}}{2}\right) & \frac{\Delta t^{2} \omega^{2}}{2 \alpha} \\
\Delta t & \Delta t-\frac{\Delta t^{3} \omega^{2}}{4 \alpha} & 2 \beta-\frac{\beta}{\alpha}-\frac{\Delta t^{2} \omega^{2} \beta}{2 \alpha} & 1-\frac{\Delta t^{2} \omega^{2}}{2 \alpha}
\end{array}\right)\left(\begin{array}{c}
{\left.[[p]]\right|_{t^{n}}} \\
p^{n,+} \\
{\left.[[q]]\right|_{t^{n}}} \\
q^{n,+}
\end{array}\right)
\end{aligned}
$$

The characteristic polynomial for the matrix $A^{n}$ has the form

$$
\left(\frac{\alpha}{\beta}-\lambda\right)\left(\frac{\beta}{\alpha}-\lambda\right)\left(\lambda^{2}+\left(\omega^{2}(\Delta t)^{2}-2\right) \lambda+1\right)=0,
$$

from which we can see that the necessary condition to satisfy the linear stability requirement $|\lambda| \leq 1$ is to take $\alpha=\beta=0.5$. Nevertheless, this restriction can be alleviated by taking $\left.[[p]]\right|_{t_{n}} \equiv 0$. In this case the first row of the transformation matrix $A^{n}$ in 3.90 , will be removed and the characteristic polynomial 3.91 reduces to

$$
\left(\frac{\beta}{\alpha}-\lambda\right)\left(\lambda^{2}+\left(\omega^{2}(\Delta t)^{2}-2\right) \lambda+1\right)=0
$$

which gives two linear stability requirements:

$$
\alpha \geq 0.5 \quad \text { and } \quad|\omega \Delta t| \leq 2 \text {. }
$$

Therefore, if $\left.[[p]]\right|_{t_{n}} \equiv 0$, a range of weights $\alpha \in[0.5,1]$ leads to a stable system under the usual Störmer-Verlet stability condition.

\section{Derivation of classical Störmer-Verlet scheme}

Considering the above mentioned requirement $\alpha \in[0.5,1],\left.[[p]]\right|_{t_{n}} \equiv 0$, we can recover the well-known form of the unforced symplectic Störmer-Verlet scheme. First, 
we rewrite 3.89 ) as

$$
\begin{aligned}
\delta q^{n,+}: & p^{n,+}=p^{n,-} \\
\delta p^{n,+}: & q^{n+1 / 2}=\beta q^{n,-}+\alpha q^{n,+}+\frac{\Delta t}{2} \frac{\partial H\left(p^{n,+}, q^{n+1 / 2}\right)}{\partial p^{n,+}} \\
\delta q^{n+1 / 2}: & p^{n+1,-}=p^{n,+}-\frac{\Delta t}{2}\left(\frac{\partial H\left(p^{n,+}, q^{n+1 / 2}\right)}{\partial q^{n+1 / 2}}+\frac{\partial H\left(p^{n+1,-}, q^{n+1 / 2}\right)}{\partial q^{n+1 / 2}}\right), \\
\delta p^{n+1,-}: & \beta q^{n+1,-}+\alpha q^{n+1,+}=q^{n+1 / 2}+\frac{\Delta t}{2} \frac{\partial H\left(p^{n+1,-}, q^{n+1 / 2}\right)}{\partial p^{n+1,-}}
\end{aligned}
$$

We see from (3.94a), that we can denote $p^{n}:=p^{n,+}=p^{n,-}$. Also, one can introduce a variable $q^{n}:=\beta q^{n,-}+\alpha q^{n,+}$, and recover the known form of the system where the first and second steps are implicit and the third step is explicit

$$
\begin{aligned}
q^{n+1 / 2} & =q^{n}+\frac{\Delta t}{2} \frac{\partial H\left(p^{n}, q^{n+1 / 2}\right)}{\partial p^{n}}, \\
p^{n+1} & =p^{n}-\frac{\Delta t}{2}\left(\frac{\partial H\left(p^{n}, q^{n+1 / 2}\right)}{\partial q^{n+1 / 2}}+\frac{\partial H\left(p^{n+1}, q^{n+1 / 2}\right)}{\partial q^{n+1 / 2}}\right) \\
q^{n+1} & =q^{n+1 / 2}+\frac{\Delta t}{2} \frac{\partial H\left(p^{n+1}, q^{n+1 / 2}\right)}{\partial p^{n+1}} .
\end{aligned}
$$

The resulting scheme is the symplectic Störmer-Verlet integrator, [64]. The charachteristic polynomial for the transformation matrix of this scheme has the form

$$
\lambda^{2}+\left(\omega^{2}(\Delta t)^{2}-2\right) \lambda+1=0,
$$

therefore the eigenvalues $\lambda$ satisfy the condition $|\lambda| \leq 1$ if $|\Delta t \omega| \leq 2$, which is the well-known stability condition for the Störmer-Verlet scheme. The dispersion relation, as discussed, for example, in [7], has the form

$$
a=-i \omega b \sqrt{1-\frac{\omega^{2} \Delta t^{2}}{4}}, \quad \hat{\omega}=\frac{\arccos \left(1-\frac{\omega^{2}(\Delta t)^{2}}{2}\right)}{\Delta t} .
$$

\subsubsection{New explicit second order variational time integration scheme}

In this section, we study the approximation introduced in Section 3.4.1. We investigate the symmetric approximation in each time slab $I_{n}$ of the variables $p$ and $q$ with Lagrangian polynomials

$$
\begin{aligned}
& p^{\tau}=\frac{t^{n+1}-t}{\Delta t} p^{n,+}+\frac{t-t^{n}}{\Delta t} p^{n+1,-}, \\
& q^{\tau}=\frac{t^{n+1}-t}{\Delta t} q^{n,+}+\frac{t-t^{n}}{\Delta t} q^{n+1,-} .
\end{aligned}
$$




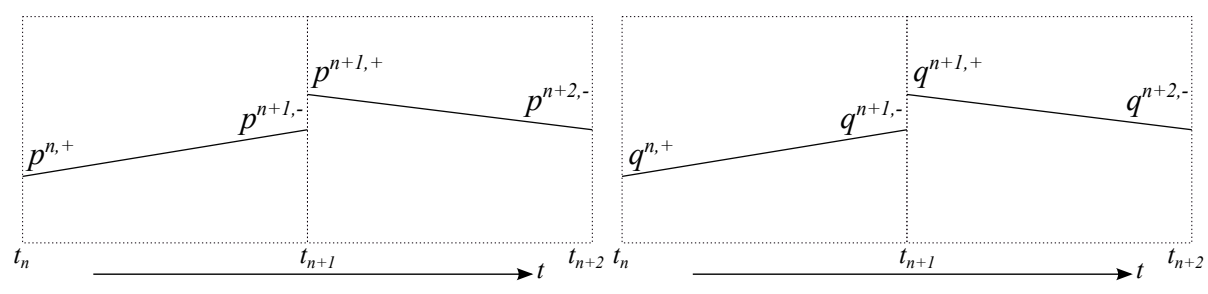

Figure 3.13: Piecewise linear approximation in time.

A symmetric, trapezoidal quadrature rule for the integral of the Hamiltonian is chosen

$$
\int_{t_{n}}^{t_{n+1}} H\left(p^{\tau}, q^{\tau}\right) \mathrm{d} t \cong \frac{\Delta t}{2}\left(H\left(p^{n,+}, q^{n,+}\right)+H\left(p^{n+1,-}, q^{n+1,-}\right)\right) .
$$

The discrete functional 3.10 is then approximated as

$$
\begin{aligned}
\mathcal{L}^{\tau}\left(p^{\tau}, q^{\tau}\right) & \cong \sum_{n=0}^{N-1}\left(\frac{1}{2}\left(p^{n,+}+p^{n+1,-}\right)\left(q^{n+1,-}-q^{n,+}\right)\right. \\
& -\frac{\Delta t}{2}\left(H\left(p^{n,+}, q^{n,+}\right)+H\left(p^{n+1,-}, q^{n+1,-}\right)\right) \\
& -\sum_{n=-1}^{N-1}\left(q^{n+1,-}-q^{n+1,+}\right)\left(\alpha p^{n+1,-}+\beta p^{n+1,+}\right) .
\end{aligned}
$$

Applying the variational principle $\delta \mathcal{L}^{\tau}=0$, using the arbitrariness of the variations, and using boundary conditions $\delta q^{0,-}=0, \delta p^{0,-}=0, \delta q^{N,+}=0, \delta p^{N,+}=0$; we obtain the second order scheme

$$
\begin{aligned}
\delta p^{n,+}: & q^{n+1,-}=(1-2 \beta) q^{n,+}+2 \beta q^{n,-}+\Delta t \frac{\partial H\left(p^{n,+}, q^{n,+}\right)}{\partial p^{n,+}}, \\
\delta q^{n,+}: & p^{n+1,-}=2 \alpha p^{n,-}+(2 \beta-1) p^{n,+}-\Delta t \frac{\partial H\left(p^{n,+}, q^{n,+}\right)}{\partial q^{n,+}}, \\
\delta p^{n+1,-}: & \alpha q^{n+1,+}=\frac{1}{2} q^{n,+}+\left(\alpha-\frac{1}{2}\right) q^{n+1,-}+\frac{\Delta t}{2} \frac{\partial H\left(p^{n+1,-}, q^{n+1,-}\right)}{\partial p^{n+1,-}}, \\
\delta q^{n+1,-}: & \beta p^{n+1,+}=\frac{1}{2} p^{n,+}+\left(\frac{1}{2}-\alpha\right) p^{n+1,-}-\frac{\Delta t}{2} \frac{\partial H\left(p^{n+1,-}, q^{n+1,-}\right)}{\partial q^{n+1,-}} .
\end{aligned}
$$

The resulting scheme consists of four explicit steps, which makes it an easy system to solve. In the next sections we will analyze the properties of this system. 


\section{Linear stability analysis}

In this section we will analyze the linear stability of the new explicit variational time integration scheme.

For the Hamiltonian, defined as $H=\frac{1}{2} \omega^{2} q^{2}+\frac{1}{2} p^{2}$ the scheme 3.101 in matrix form becomes

$$
\begin{aligned}
& \left(\begin{array}{l}
p^{n+1,-} \\
q^{n+1,-} \\
p^{n+1,+} \\
q^{n+1,+}
\end{array}\right)=A\left(\begin{array}{l}
p^{n,-} \\
q^{n,-} \\
p^{n,+} \\
q^{n,+}
\end{array}\right) \\
& =\left(\begin{array}{c:ccc}
2 \alpha & 0 & 2 \beta-1 & -\Delta t \omega^{2} \\
0 & 2 \beta & 0 t & 1-2 \beta \\
(\beta-\alpha) \frac{\alpha}{\beta} & -\Delta t \omega^{2} & \frac{1}{\beta}-2 \alpha-\frac{\omega^{2}(\Delta t)^{2}}{2 \beta} & 0 \\
\Delta t & (\alpha-\beta) \frac{\beta}{\alpha} & 0 & \frac{1}{\alpha}-2 \beta-\frac{\omega^{2}(\Delta t)^{2}}{2 \alpha}
\end{array}\right)\left(\begin{array}{l}
p^{n,-} \\
q^{n,-} \\
p^{n,+} \\
q^{n,+}
\end{array}\right) .
\end{aligned}
$$

We compute the eigenvalues of the matrix $A$ numerically by taking a range of weights $\alpha$ and frequencies multiplied by a time step, i.e. $\omega \Delta t$. Also, the relation $\beta=1-\alpha$ is used. In Figure 3.14 shadowed area in the $\alpha-\omega \Delta t$ plane presents the domain where the maximum modulus of the eigenvalue is equal to one. In fact, within the stability domain all the eigenvalues are equal to one in modulus. As can be seen from this figure, in order

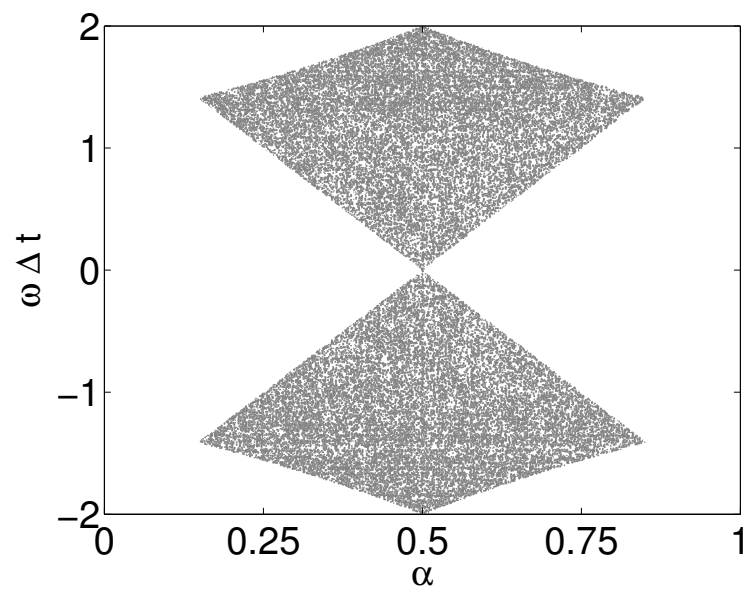

Figure 3.14: Modulus of eigenvalues $\lambda_{i}$ of system 3.102 for a linear harmonic oscillator. The shadowed area corresponds to the region where the modulus of all the eigenvalues is equal to one.

to ensure linear stability around $\omega \Delta t=0$, one needs to take the weight $\alpha=0.5$. In this 
case the characteristic polynomial takes the form

$$
\left(\lambda^{2}+\left(\omega^{2}(\Delta t)^{2}-2\right) \lambda+1\right)^{2}=0,
$$

which is the squared characteristic polynomial 3.96 for the Störmer-Verlet scheme. The eigenvalues are therefore the same as for the Störmer-Verlet scheme, and $|\lambda| \leq 1$ if $|\Delta t \omega| \leq 2$, so the stability condition for the new system is obtained and it coincides with the linear stability condition for the Störmer-Verlet scheme.

Substituting $\alpha=0.5, \beta=0.5$ in 3.101 we obtain the straightforward explicit scheme

$$
\begin{aligned}
& q^{n+1,-}=q^{n,-}+\Delta t \frac{\partial H\left(p^{n,+}, q^{n,+}\right)}{\partial p^{n,+}}, \\
& p^{n+1,-}=p^{n,-}-\Delta t \frac{\partial H\left(p^{n,+}, q^{n,+}\right)}{\partial q^{n,+}}, \\
& q^{n+1,+}=q^{n,+}+\Delta t \frac{\partial H\left(p^{n+1,-}, q^{n+1,-}\right)}{\partial p^{n+1,-}}, \\
& p^{n+1,+}=p^{n,+}-\Delta t \frac{\partial H\left(p^{n+1,-}, q^{n+1,-}\right)}{\partial q^{n+1,-}},
\end{aligned}
$$

which is a new four stage explicit scheme. This property is desirable for computations since other considered schemes are (partly) implicit.

\section{Order of accuracy of four stage explicit scheme}

A numerical convergence test for the variables $p^{n+1,+}, q^{n+1,+}$ shows that the scheme 3.104 is only first order accuracy, while a convergence test for the averaged variables $\frac{1}{2}\left(p^{n+1,-}+p^{n+1,+}\right), \frac{1}{2}\left(q^{n+1,-}+q^{n+1,+}\right)$ shows second order accuracy. In this section we will explain this behavior analytically.

We approximate a system with two variables $p$ and $q$ via a system with four variables $p^{-}, p^{+}$and $q^{-}, q^{+}$. The analysis of the order of accuracy needs to be changed for extended schemes therefore. Here we discuss the analysis for the new second order scheme 3.104 and give an explanation of the convergence issues related to it. To make the analysis simpler, we consider the harmonic oscillator with $H=\frac{1}{2} p^{2}+\frac{1}{2} \omega^{2} q^{2}$. We also split the system into two separate parts:

$$
\begin{aligned}
& p^{n+1,-}=p^{n,-}-\Delta t \omega^{2} q^{n,+}, \\
& q^{n+1,+}=\left(1-\omega^{2}(\Delta t)^{2}\right) q^{n,+}+\Delta t p^{n,-},
\end{aligned}
$$

and

$$
\begin{aligned}
& q^{n+1,-}=q^{n,-}+\Delta t p^{n,+}, \\
& p^{n+1,+}=\left(1-\omega^{2}(\Delta t)^{2}\right) p^{n,+}-\Delta t \omega^{2} q^{n,-} .
\end{aligned}
$$


We will analyze first each system separately. In this analysis it is useful to remember the partial differential equations that we are solving here:

$$
q_{t}=p, p_{t}=-\omega^{2} q
$$

which can be combined into

$$
p_{t t}+\omega^{2} p=0, q_{t t}+\omega^{2} q=0 .
$$

We start with system 3.105. Consider a Taylor expansion of 3.105a, 3.105b

$$
\begin{aligned}
& p^{-}\left(t^{n}\right)+\Delta t\left(p^{-}\right)_{t}\left(t^{n}\right)+\frac{\Delta t^{2}}{2}\left(p^{-}\right)_{t t}\left(t^{n}\right)+O\left(\Delta t^{3}\right) \\
&= p^{-}\left(t^{n}\right)-\Delta t \omega^{2} q^{+}\left(t^{n}\right), \\
& q^{+}\left(t^{n}\right)+\Delta t\left(q^{+}\right)_{t}\left(t^{n}\right)+\frac{\Delta t^{2}}{2}\left(q^{+}\right)_{t t}\left(t^{n}\right)+O\left(\Delta t^{3}\right) \\
&=\left(1-\omega^{2}(\Delta t)^{2}\right) q^{+}\left(t^{n}\right)+\Delta t p^{-}\left(t^{n}\right),
\end{aligned}
$$

which after dropping $t^{n}$ and employing the notation $\left(p^{-}\right)_{t}=p_{t}^{-}$can be rearranged into

$$
\begin{aligned}
& p_{t}^{-}+\frac{\Delta t}{2} p_{t t}^{-}+\omega^{2} q^{+}+O\left(\Delta t^{2}\right)=0, \\
& q_{t}^{+}+\frac{\Delta t}{2} q_{t t}^{+}+\omega^{2} \Delta t q^{+}-p^{-}+O\left(\Delta t^{2}\right)=0 .
\end{aligned}
$$

As a result the variables $p^{n,-}, q^{n,+}$ approximate the continuum variables $p^{-}, q^{+}$in equations 3.107) with first order accuracy only. In the same way we analyze the second system (3.106). After some rearrangements we have

$$
\begin{aligned}
& q_{t}^{-}+\frac{\Delta t}{2} q_{t t}^{-}-p^{+}+O\left(\Delta t^{2}\right)=0 \\
& p_{t}^{+}+\frac{\Delta t}{2} p_{t t}^{+}+\omega^{2} \Delta t p^{+}+\omega^{2} q^{-}+O\left(\Delta t^{2}\right)=0 .
\end{aligned}
$$

The same conclusion as for the first system holds: the numerical approximation is only first order accurate.

Nevertheless, remember, that the two systems need to be considered together. After summation of 3.105a, 3.105b), 3.106a and 3.106b we obtain

$$
\begin{aligned}
& p^{n+1,-}+p^{n+1,+}+q^{n+1,+}+q^{n+1,-} \\
& =p^{n,-}-\Delta t \omega^{2} q^{n,+}+\left(1-\omega^{2}(\Delta t)^{2}\right) q^{n,+}+\Delta t p^{n,-} \\
& +q^{n,-}+\Delta t p^{n,+}+\left(1-\omega^{2}(\Delta t)^{2}\right) p^{n,+}-\Delta t \omega^{2} q^{n,-} .
\end{aligned}
$$


Define $p^{n}=\frac{1}{2}\left(p^{n,-}+p^{n,+}\right), q^{n}=\frac{1}{2}\left(q^{n,-}+q^{n,+}\right)$ and reformulate 3.111, as

$$
p^{n+1}+q^{n+1}=p^{n}-\Delta t \omega^{2} q^{n}+\Delta t p^{n}+q^{n}-\omega^{2}(\Delta t)^{2}\left(p^{n,+}+q^{n,+}\right) .
$$

Next, we use the Taylor expansion and get

$$
\begin{aligned}
p\left(t^{n}\right) & +\Delta t p_{t}\left(t^{n}\right)+\frac{\Delta t^{2}}{2} p_{t t}\left(t^{n}\right)+q\left(t^{n}\right)+\Delta t q_{t}\left(t^{n}\right)+\frac{\Delta t^{2}}{2} q_{t t}\left(t^{n}\right)+O\left(\Delta t^{3}\right) \\
& =p\left(t^{n}\right)-\Delta t \omega^{2} q\left(t^{n}\right)+\Delta t p\left(t^{n}\right)+q\left(t^{n}\right)-\omega^{2} \frac{(\Delta t)^{2}}{2}\left(p^{+}+q^{+}\right),
\end{aligned}
$$

which can be simplified after dropping $t^{n}$ into

$$
\begin{aligned}
& \Delta t\left(p_{t}+\omega^{2} q\right)+\Delta t\left(q_{t}-p\right) \\
& +\frac{\Delta t^{2}}{2}\left(p_{t t}+\omega^{2} p^{+}\right)+\frac{\Delta t^{2}}{2}\left(q_{t t}+\omega^{2} q^{+}\right)+O\left(\Delta t^{3}\right)=0 .
\end{aligned}
$$

This relation can be further simplified using the jump notation $[d]=d^{-}-d^{+}$

$$
\begin{aligned}
& \Delta t\left(p_{t}+\omega^{2} q\right)+\Delta t\left(q_{t}-p\right) \\
& +\frac{\Delta t^{2}}{2}\left(p_{t t}+\omega^{2} p-\frac{\omega^{2}}{2}[p]\right)+\frac{\Delta t^{2}}{2}\left(q_{t t}+\omega^{2} q-\frac{\omega^{2}}{2}[q]\right)+O\left(\Delta t^{3}\right)=0 .
\end{aligned}
$$

For smooth solutions one can assume that the jump is $O(\triangle t)^{2}$ when we use linear polynomials. Then from relation 3.115) we observe that the scheme (3.104) approximates the system of equations 3.107 with second order accuracy if the final result of each time step is defined as

$$
p^{n+1}=\frac{1}{2}\left(p^{n+1,-}+p^{n+1,+}\right), \quad q^{n+1}=\frac{1}{2}\left(q^{n+1,-}+q^{n+1,+}\right) .
$$

\section{Dispersion analysis}

Since we have an extended system with four variables to approximate a two-variable system, it is necessary to use a special technique to compute the dispersion error. First, we use that for the simple case of a harmonic oscillator with the separable Hamiltonian 3.102 (where $\alpha=0.5$ ) the system can be split into two separate systems, viz. 3.105) and (3.106). In system 3.105 the variables $p^{n+1,-}$ and $q^{n+1,+}$ are conjugated, and in system (3.106) this applies to $p^{n+1,+}$ and $q^{n+1,-}$. This makes the system somewhat analogous to the continuous system given in Appendix 3.7.4 We will analyze both systems 3.105 and 3.106 separately.

We start with system 3.105. Consider the Fourier modes

$$
\left(\begin{array}{c}
p^{n,-} \\
q^{n,+}
\end{array}\right)=\left(\begin{array}{l}
a e^{-i \hat{\omega} n \Delta t} \\
b e^{-i \hat{\omega} n \Delta t}
\end{array}\right)
$$


and substitute them into 3.105 . After dividing by the factor $e^{-i \hat{\omega} n \Delta t}$ the system becomes

$$
\begin{aligned}
& a(\cos (\hat{\omega} \Delta t)-i \sin (\hat{\omega} \Delta t))=a-\Delta t \omega^{2} b, \\
& b(\cos (\hat{\omega} \Delta t)-i \sin (\hat{\omega} \Delta t))=\left(1-\omega^{2}(\Delta t)^{2}\right) b+\Delta t a,
\end{aligned}
$$

and one arrives at

$$
\begin{aligned}
a & =\frac{b \omega^{2} \Delta t \pm i b \omega \sqrt{4-\omega^{2} \Delta t^{2}}}{2}, \\
\cos (\hat{\omega} \Delta t) & =1-\frac{\omega^{2}(\Delta t)^{2}}{2} .
\end{aligned}
$$

Therefore the discrete amplitude relation converges to the exact relation $a=-i \omega b$ when the minus sign is chosen; and the discrete frequency $\hat{\omega}$ equals the discrete frequency of the Störmer-Verlet scheme.

In order to simplify the discrete amplitude relation 3.119a, we introduce the frequency $\theta$ as

$$
\cos \theta=\frac{\sqrt{4-\omega^{2} \Delta t^{2}}}{2}, \quad \sin \theta=\frac{\omega \Delta t}{2} .
$$

Relation 3.119a for a positive sign then becomes

$$
a=i b \omega e^{-\theta i},
$$

or for the negative sign

$$
a=-i b \omega e^{\theta i}
$$

The same analysis holds for system 3.106 . Consider the Fourier modes

$$
\left(\begin{array}{l}
p^{n,+} \\
q^{n,-}
\end{array}\right)=\left(\begin{array}{l}
c e^{-i \hat{\omega} n \Delta t} \\
d e^{-i \hat{\omega} n \Delta t}
\end{array}\right) .
$$

After substitution of these relations into 3.106 we obtain

$$
\begin{aligned}
c & =\frac{-d \omega^{2} \Delta t \pm i d \omega \sqrt{4-\omega^{2} \Delta t^{2}}}{2}, \\
\cos (\hat{\omega} \Delta t) & =1-\frac{\omega^{2}(\Delta t)^{2}}{2},
\end{aligned}
$$

where 3.124a can also be expressed as

$$
c=i d \omega e^{\theta i}
$$


or

$$
c=-i d \omega e^{-\theta i}
$$

Naturally, neither system 3.105 with the conjugated variables $p^{-}, q^{+}$nor the system 3.106 with $p^{+}, q^{-}$can be considered as the final result. Moreover, as shown in the previous subsection 3.7.3. one needs to take the average of the left and right trace at the time level $t^{n}$ to obtain second order accuracy. Therefore if we consider, for example, the average variable $p^{n}$, constructed using the coefficients $a(\sqrt{3.121})$ - negative mode or 3.122 - positive mode) and $c$ (3.125 - negative mode or 3.126 - positive mode), we might obtain a combination of positive and negative modes in one variable.

The explicit second order accurate scheme is stable for linear problems, but for nonlinear systems (e.g. nonlinear water waves) the energy error tends to grow. The solution, however, does not show an instability.

\section{Symplecticity}

One can verify that the condition 3.47 is valid for the new system 3.104. The transformation matrix for the new scheme has the form

$$
\begin{aligned}
& \frac{\partial\left(p^{n+1,-}, p^{n+1,+}, q^{n+1,-}, q^{n+1,+}\right)}{\partial\left(p^{n,-}, p^{n,+}, q^{n,-}, q^{n,+}\right)} \\
& =\left(\begin{array}{cccc}
1 & -\Delta t H_{p q}^{n,+} & 0 & -\Delta t H_{q q}^{n,+} \\
-\Delta t H_{p q}^{n+1,-} & a & -\Delta t H_{q q}^{n+1,-} & b \\
0 & \Delta t H_{p p}^{n,+} & 1 & \Delta t H_{p q}^{n,+} \\
\Delta t H_{p p}^{n+1,-} & c & \Delta t H_{p q}^{n+1,-} & d
\end{array}\right),
\end{aligned}
$$

with the notations

$$
\begin{aligned}
& a=1+(\Delta t)^{2} H_{p q}^{n+1,-} H_{p q}^{n,+}-(\Delta t)^{2} H_{q q}^{n+1,-} H_{p p}^{n,+}, \\
& b=(\Delta t)^{2} H_{p q}^{n+1,-} H_{q q}^{n,+}-(\Delta t)^{2} H_{p q}^{n,+} H_{q q}^{n+1,-}, \\
& c=(\Delta t)^{2} H_{p q}^{n+1,-} H_{p p}^{n,+}-(\Delta t)^{2} H_{p p}^{n+1,-} H_{p q}^{n,+}, \\
& d=1-(\Delta t)^{2} H_{p p}^{n+1,-} H_{q q}^{n,+}+(\Delta t)^{2} H_{p q}^{n+1,-} H_{p q}^{n,+},
\end{aligned}
$$

and $H_{p p}^{n,+}=H_{p p}\left(p^{n,+}, q^{n,+}\right), H_{p p}^{n+1,-}=H_{p p}\left(p^{n+1,-}, q^{n+1,-}\right)$ etc.

\subsubsection{Extended continuum system}

Since in the time discontinuous schemes we are working with extended systems, we also need to extend the analysis for the continuous case. For example, in the extended system for the harmonic oscillator model problem, we have four initial conditions $\left(p^{0,-}, p^{0,+}, q^{0,-}, q^{0,+}\right)$, instead of two for the classical schemes $\left(p^{0}, q^{0}\right)$, and four (instead of two) variables need to be updated every time step. 
For the new second order scheme (3.104) with two pairs of coupled variables, we can construct an analogous extended Lagrangian functional as

$$
\mathcal{L}(p, q):=\int_{0}^{T}\left(p^{+} \frac{\mathrm{d} q^{-}}{\mathrm{d} t}+p^{-} \frac{\mathrm{d} q^{+}}{\mathrm{d} t}-H\left(p^{+}, q^{-}\right)-H\left(p^{-}, q^{+}\right)\right) \mathrm{d} t
$$

with the Hamiltonian defined as

$$
H(p, q):=\frac{1}{2} p^{2}+\frac{1}{2} \omega^{2} q^{2} .
$$

Applying the variational principle, integrating 3.130 by parts, using the end point conditions $\delta q^{-}(0)=\delta q^{-}(T)=0, \delta q^{+}(0)=\delta q^{+}(T)=0$, and the arbitrariness of the variations; the equations for the extended system emerge as follows:

$$
\begin{array}{lll}
\frac{\mathrm{d} q^{+}}{\mathrm{d} t}-\frac{\delta H}{\delta p^{-}}=0 & \text { and } & \frac{\mathrm{d} p^{-}}{\mathrm{d} t}+\frac{\delta H}{\delta q^{+}}=0, \\
\frac{\mathrm{d} q^{-}}{\mathrm{d} t}-\frac{\delta H}{\delta p^{+}}=0 & \text { and } & \frac{\mathrm{d} p^{+}}{\mathrm{d} t}+\frac{\delta H}{\delta q^{-}}=0 .
\end{array}
$$

For the harmonic oscillator 3.131) these equations reduce to

$$
\begin{array}{lll}
q_{t}^{-}=p^{+} & \text {and } & p_{t}^{+}=-\omega^{2} q^{-}, \\
q_{t}^{+}=p^{-} & \text {and } & p_{t}^{-}=-\omega^{2} q^{+} .
\end{array}
$$

The system 3.133 can be represented in matrix form as

$$
\left(\begin{array}{l}
p^{-} \\
q^{-} \\
p^{+} \\
q^{+}
\end{array}\right)_{t}=\left(\begin{array}{cccc}
0 & 0 & 0 & -\omega^{2} \\
0 & 0 & 1 & 0 \\
0 & -\omega^{2} & 0 & 0 \\
1 & 0 & 0 & 0
\end{array}\right)\left(\begin{array}{l}
p^{-} \\
q^{-} \\
p^{+} \\
q^{+}
\end{array}\right)=A\left(\begin{array}{l}
p^{-} \\
q^{-} \\
p^{+} \\
q^{+}
\end{array}\right)
$$

The characteristic polynomial for the system matrix is

$$
\left(\lambda^{2}+\omega^{2}\right)^{2}=0
$$

which is the square of the relation for the simple oscillator 3.84. Therefore the eigenvalues are the same as or the simple oscillator.

We consider now the extended system. The exact solution has the form

$$
\left(\begin{array}{l}
p^{-} \\
q^{-} \\
p^{+} \\
q^{+}
\end{array}\right)=\left(\begin{array}{cccc}
\cos (\omega t) & 0 & 0 & -\omega \sin (\omega t) \\
0 & \cos (\omega t) & \frac{1}{\omega} \sin (\omega t) & 0 \\
0 & -\omega \sin (\omega t) & \cos (\omega t) & 0 \\
\frac{1}{\omega} \sin (\omega t) & 0 & 0 & \cos (\omega t)
\end{array}\right)\left(\begin{array}{l}
p^{0,-} \\
q^{0,-} \\
p^{0,+} \\
q^{0,+}
\end{array}\right)
$$


By analogy with system 3.85 we can find the dispersion relation by taking Fourier modes

$$
\begin{aligned}
& \left(\begin{array}{l}
p^{-} \\
q^{-} \\
p^{+} \\
q^{+}
\end{array}\right)=\operatorname{Re}\left(\begin{array}{c}
a e^{-i \hat{\omega} t} \\
b e^{-i \hat{\omega} t} \\
c e^{-i \hat{\omega} t} \\
d e^{-i \hat{\omega} t}
\end{array}\right) \\
& =\left(\begin{array}{cccc}
\cos (\hat{\omega} t) & 0 & 0 & \frac{\operatorname{Im}(a)}{\operatorname{Re}(d)} \sin (\hat{\omega} t) \\
0 & \cos (\hat{\omega} t) & \frac{\operatorname{Im}(b)}{\operatorname{Re}(c)} \sin (\hat{\omega} t) & 0 \\
0 & \frac{\operatorname{Im}(c)}{\operatorname{Re}(b)} \sin (\hat{\omega} t) & \cos (\hat{\omega} t) & 0 \\
\frac{\operatorname{Im}(d)}{\operatorname{Re}(a)} \sin (\hat{\omega} t) & 0 & 0 & \cos (\hat{\omega} t)
\end{array}\right)\left(\begin{array}{l}
p^{0,-} \\
q^{0,-} \\
p^{0,+} \\
q^{0,+}
\end{array}\right) .
\end{aligned}
$$

Comparing 3.137 to the exact solution 3.136 we obtain a system of equations that provides the dispersion relations

$$
\hat{\omega}=\omega, \quad c=-i \omega b, \quad a=-i \omega d,
$$

or

$$
\hat{\omega}=-\omega, \quad c=i \omega b, \quad a=i \omega d .
$$




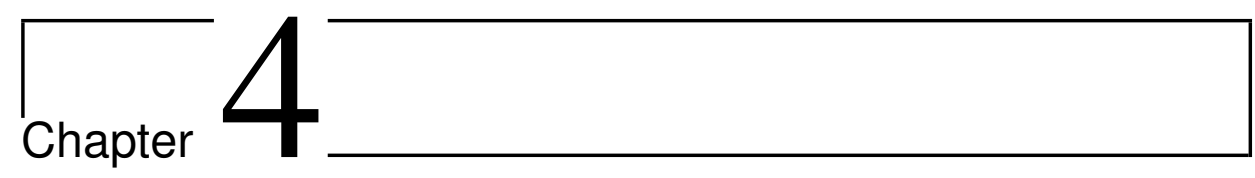

\section{Variational space-time (dis)continuous Galerkin method for nonlinear free surface water}

\section{waves}

A new variational finite element method is developed for nonlinear free surface gravity water waves using the potential flow approximation. This method also handles waves generated by a wave maker. Its formulation stems from Miles' variational principle for water waves together with a finite element discretization that is continuous in space and discontinuous in time. One novel feature of this variational finite element approach is that the free surface evolution is variationally dependent on the mesh deformation vis-à-vis the mesh deformation is geometrically dependent on free surface evolution. Another key feature is the use of a variational (dis)continuous Galerkin finite element discretization in time. Moreover, in the absence of a wave maker, it is shown to be equivalent to the second order symplectic Störmer-Verlet time stepping scheme for the free-surface degrees of freedom. These key features add to the stability of the numerical method. Finally, the resulting numerical scheme is verified against nonlinear analytical solutions with long time simulations and validated against experimental measurements of driven wave solutions in a wave basin of the Maritime Research Institute Netherlands.

The contents of the chapter are partly presented as a journal article [53] written in collaboration with V. Ambati, J.J.W. van der Vegt and O. Bokhove. 


\subsection{Introduction}

The hydrodynamics of water waves in offshore regions of seas and oceans are of significant interest to naval architects and marine engineers. Particularly important is the dynamics of extreme wave phenomena, such as rogue waves, and their effect on offshore structures and ships. Engineers often test prototype models of ships and offshore structures in experimental wave basins with wave makers to mimic realistic maritime environments. For extreme waves it is nontrivial to generate waves with specific properties at certain measurement locations in a wave basin. Numerical modeling of waves is therefore an important complementary activity.

A class of water wave problems is described by the Laplace equation for the velocity potential with Neumann and nonlinear free surface boundary conditions. These free surface gravity wave equations are obtained from the Euler equations of fluid motion under the assumptions that the fluid is inviscid and incompressible, and the velocity field irrotational [72].

Free surface gravity water wave equations can also be obtained in a succinct way via Luke's variational principle [98] or from its dynamical equivalent presented by Miles [106]. In the variational principle, the complete problem is embedded in a single functional. In addition, the variational formulation is associated with conservation of energy and phase space. Improved finite element solutions of the free surface water wave equations can be constructed directly from this variational formulation rather than from a separate weak formulation. Since variational finite element formulations have as main benefit that they provide a natural setting to ensure discrete energy and phase space conservation, they can more naturally lead to stable numerical schemes.

Variational and finite element formulations for free surface waves using potential flow can be found in Kim and Bai [78] and Kim et al. [79]. Their formulation is, however, restricted to an unbounded domain and involves a coordinate transformation to a reference domain. Klopman et al. [82] derived a variational Boussinesq model from Miles' variational principle (see also [30, 54]). More, standard finite element methods for free surface gravity water waves can be found in [99, 100, 101, 149, 151, 152, 156].

Another widely used numerical method for free surface waves using potential flow is the boundary integral method, which started with the work of Longuet-Higgins and Cokelet [97], and Vinje and Brevig [146]. This method has been applied extensively to two dimensional free surface waves, see the surveys of Romate and Zandbergen [129] and Tsai and Yue [142]. Applications in three dimensions (3D) using boundary integral methods are widespread [9, 21, 50, 51, 62, 69]. A comparison between the work load of boundary and finite element methods is made in Bettess [11], with finite element methods being competitive in anisotropic domains.

More recently, discontinuous Galerkin (DG) methods for elliptic problems have enabled researchers to model free surface water waves with DG methods (see the overview in Arnold et al. [5]). In particular, space-time DG methods, in which time is considered as an additional coordinate and the problem is solved in $d+1$ space, with $d$ the spatial 
dimension, are promising for free surface flows with their deforming domains. Space(time) DG finite element methods for linear free surface wave problems were developed in Van der Vegt and Tomar [145] and for nonlinear flows in Van der Vegt and Xu [144].

Numerically solving the nonlinear free surface gravity wave equations poses a number of challenges:

(i) the solution of the governing equations depends on the position of the free surface, which is not known a priori;

(ii) the flow domain continuously deforms in time, not only due to the free surface motion, but also due to the presence of a wave maker;

(iii) energy drift needs to be avoided in time in order to minimize numerical decay of wave amplitude (in the unforced limit); and,

(iv) dispersion errors need to be small for accurate capturing of complex wave interactions.

To cope with these challenges, we developed a variational finite element method for nonlinear free surface gravity water waves based on Miles' variational principle.

In our space-plus-time approach, space is discretized first by considering continuous basis functions in (space). These are substituted directly into Miles' variational principle. The resulting space discrete variational principle is either autonomous (i.e., with no explicit time dependence) or non-autonomous (i.e., including the explicit time dependence due to the wave maker) in time. Subsequently, we develop a time (dis)continuous Galerkin approach by using discontinuous basis functions in time to discretize this spatially discrete variational principle, by first eliminating the interior degrees of freedom. The result is that the free surface becomes continuous while the (surface) velocity potential remains discontinuous in time. A posteriori, we also establish the time discretization of the interior degrees of freedom. The final result is a space and time discrete variational principle.

By taking variations of this space(-time) discrete variational principle, a system of nonlinear ordinary-differential or algebraic equations for the free surface height and the velocity potential is obtained. Besides directly discretizing the variational principle, the central key features that make this discretization robust and stable are as follows:

(i) Variations of the positions of interior nodes of the dynamic mesh must be related variationally to the free surface nodes. If one removes this intrinsic dependence the resulting numerical scheme is prone to be unstable.

(ii) The new variational time discretization deals with the intrinsically coupled dynamical system, both in the autonomous and non-autonomous cases, the latter with driven waves. 
These two features appear to contrast with earlier (DG) finite element approaches, and result in a stable numerical scheme with no drift of the discrete energy and preservation of discrete phase space.

For a certain linear approximation of the flow fields in time, our space-time variational finite element method results in a time discretization similar to the symplectic StörmerVerlet time discretization. In the autonomous case without wave maker, we show that it is a Störmer-Verlet time discretization [64] of the dynamics at the free surface. The latter dynamics constitute a standard yet nonlinear canonical Hamiltonian system (of ordinary differential equations before time is discretized as well). The first two steps in the time integration scheme are implicit, and the third step is explicit. Hence, the variational finite element discretization leads to a system of nonlinear algebraic equations. It is solved by a Newton method yielding a sparse matrix. The sparsity depends directly on the number of nodes surrounding each node in the flow domain. The sparse matrix storage routines and sparse iterative or direct solvers of the PETSc package are used [131, 132, 133].

Our variational finite element method is verified against a nonlinear semi-analytical solution by Fenton and Rienecker [47] for horizontally periodic waves and validated against experimental data sets provided by the Maritime Research Institute Netherlands (MARIN). These experiments are conducted using two wave basin configurations, one with a varying bottom and one with a flat bottom. In both cases, waves are generated with a piston type wave maker at one end of the domain. This experimental setup can lead to highly irregular waves as well as wave focussing, in which a slow moving wave train is overtaken by a fast moving wave train. More information on the experiments can be found in [68] and another numerical validation in Latifah and Van Groesen [91].

The outline of the chapter is as follows. In Section 4.2, we present Miles' variational principle for nonlinear free surface waves and derive the governing equations. In Section 4.3 the variational finite element method is formulated. Subsequently, the numerical verification and experimental validation are discussed in Section 4.4. A novel third order time integrator derived in Chapter 3 is implemented for an autonomous case in Section 4.5.1. Finally, conclusions are drawn in Section 4.6 .

\subsection{Variational Description of Nonlinear Inviscid Water Waves}

We consider water waves generated by a wave maker in a two-dimensional wave basin in a vertical plane. This restriction to two dimensions is not essential to our approach. The fluid is assumed to be inviscid, irrotational and incompressible. A sketch of the wave basin is shown in Fig. 4.1 .

The dynamics of water waves are governed by the following variational principle (cf., 


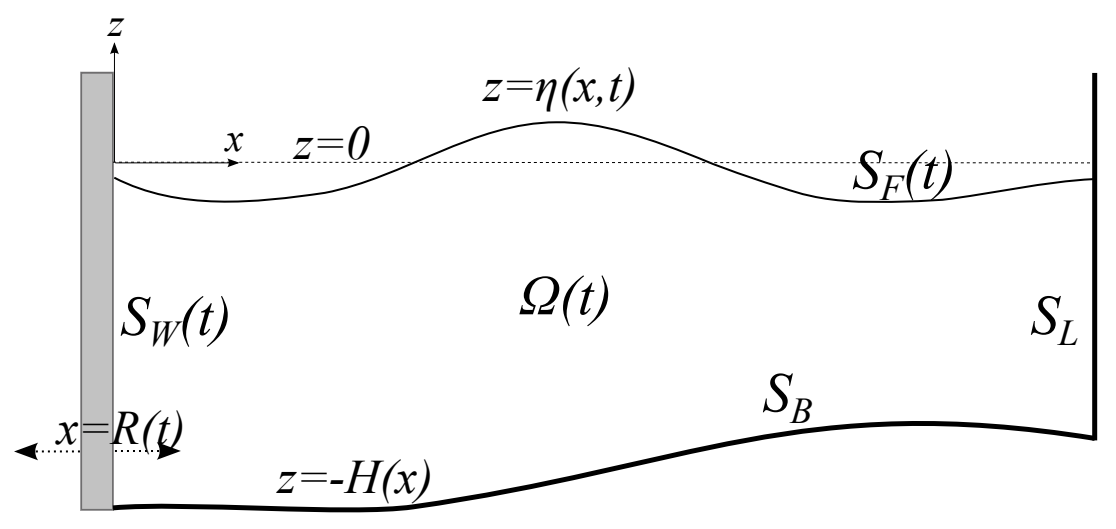

Figure 4.1: Sketch of the numerical wave basin with water waves generated by a piston wave maker.

Miles [106]):

$$
\begin{aligned}
0= & \delta \mathcal{L}\left(\phi, \eta, \phi_{s}\right) \mathrm{d} t:=\delta \int_{0}^{T}\left(\int_{R(t)}^{L}\left(\phi_{s} \frac{\partial \eta}{\partial t}-\frac{1}{2} g\left(\eta^{2}-H^{2}\right)\right) \mathrm{d} x\right. \\
& \left.-\int_{R(t)}^{L} \int_{-H(x)}^{\eta(x, t)} \frac{1}{2}|\nabla \phi|^{2} \mathrm{~d} z \mathrm{~d} x-\int_{-H(R(t))}^{\eta(R(t), t)} \frac{\mathrm{d} R}{\mathrm{~d} t} \phi_{w} \mathrm{~d} z\right) \mathrm{d} t
\end{aligned}
$$

where $(x, z)$ are the horizontal and vertical coordinates, respectively, $\nabla$ is the gradient operator in the vertical plane, $\phi(x, z, t)$ the velocity potential, $\mathbf{u}:=\nabla \phi$ the irrotational velocity field, and $\Omega=\{R(t)<x<L,-H(x)<z<\eta(x, t)\} \subset \mathbb{R}^{2}$ the time dependent flow domain. The boundaries of $\Omega$ are the free surface boundary $S_{F}: z=\eta(x, t)$ with $\eta$ the free surface height, the fixed bed at the bottom $S_{B}: z=-H(x)(z=0$ is the fixed reference level of the free surface at rest), a piston wave maker $S_{W}: x=R(t)$ and a solid wall $S_{L}: x=L$. For brevity's sake, we define $\phi_{s}(x, t)=\phi(x, \eta(x, t), t), \phi_{w}(z, t)=$ $\phi(R(t), z, t), \eta_{w}(t)=\eta(R(t), t), H_{w}=H(R(t))$ and $\phi_{s w}(t)=\phi(R(t), \eta(R(t), t), t)$. Note that the wave maker moves only horizontally and that the free surface is assumed to be singly-valued. 
Taking the variational derivatives with respect to $\phi, \eta$ and $\phi_{s}$ in (4.1) yields

$$
\begin{aligned}
0= & \delta \mathcal{L}=\int_{0}^{T}\left(-\int_{\Omega(t)} \nabla \phi \cdot \nabla \delta \phi \mathrm{d} x \mathrm{~d} z-\int_{-H_{w}(t)}^{\eta_{w}(t)} \frac{\mathrm{d} R}{\mathrm{~d} t} \delta \phi_{w} \mathrm{~d} z\right. \\
& \left.+\int_{R(t)}^{L}\left(\frac{\partial \eta}{\partial t} \delta \phi_{s}+\phi_{s} \frac{\partial \delta \eta}{\partial t}-\left(g \eta+\frac{1}{2}|\nabla \phi|_{s}^{2}\right) \delta \eta\right) \mathrm{d} x-\frac{\mathrm{d} R}{\mathrm{~d} t} \phi_{s w} \delta \eta_{w}\right) \mathrm{d} t .
\end{aligned}
$$

We simply took the variations with respect to the time-dependent boundary $z=\eta$ of the domain $\Omega(t)$, but could alternatively have used a variational analogue of the Reynolds transport theorem as in [54]. Integrating [4.2) by parts in space using Gauss' divergence theorem as well as integrating the term $\phi_{s} \partial \delta \eta / \partial t$ in 4.2 by parts in time using Leibnitz' rule yields

$$
\begin{aligned}
0= & \delta \mathcal{L}=\int_{0}^{T}\left(\int_{\Omega(t)} \nabla^{2} \phi \delta \phi \mathrm{d} x \mathrm{~d} z-\int_{\partial \Omega(t)} \mathbf{n} \cdot \nabla \phi \delta \phi \mathrm{d} S-\int_{-H_{w}(t)}^{\eta_{w}(t)} \frac{\mathrm{d} R}{\mathrm{~d} t} \delta \phi_{w} \mathrm{~d} z\right. \\
& \left.+\int_{R(t)}^{L}\left(\frac{\partial \eta}{\partial t} \delta \phi_{s}-\left(\frac{\partial \phi_{s}}{\partial t}+g \eta+\frac{1}{2}|\nabla \phi|_{s}^{2}\right) \delta \eta\right) \mathrm{d} x+\left.\int_{R(t)}^{L} \phi_{s} \delta \eta \mathrm{d} x\right|_{0} ^{T}\right) \mathrm{d} t
\end{aligned}
$$

with $\partial \Omega:=S_{F} \cup S_{B} \cup S_{W} \cup S_{L}$ and $\mathbf{n}$ the outward unit normal vector at the boundary $\partial \Omega$, and $\mathrm{d} S$ an infinitesimal line element along such a boundary. Due to the integration by parts in time an additional term arises such that the last term in $(4.2)$, the point at the free surface and wave-maker, cancels. By using the relations

$$
\begin{aligned}
\delta \phi_{s} & =(\delta \phi)_{s}+(\partial \phi / \partial z)_{s} \delta \eta, \\
\int_{S_{F}} \mathrm{~d} S & =\int_{R(t)}^{L}|\nabla(z-\eta)| \mathrm{d} x, \\
\left.\mathbf{n}\right|_{S_{F}} & =\nabla(z-\eta) /|\nabla(z-\eta)|, \\
\delta \eta(x, 0) & =\delta \eta(x, T)=0
\end{aligned}
$$

and after splitting the boundary integrals over the different types of boundary faces, the 
variations in 4.3 become

$$
\begin{aligned}
0=\delta \mathcal{L}= & \int_{0}^{T}\left(\int_{\Omega(t)} \nabla^{2} \phi \delta \phi \mathrm{d} x \mathrm{~d} z-\int_{S_{B} \cup S_{L}} \mathbf{n} \cdot \nabla \phi \delta \phi \mathrm{d} S\right. \\
& -\int_{-H_{w}(t)}^{\eta_{w}(t)}\left(\frac{\mathrm{d} R}{\mathrm{~d} t}-\left(\frac{\partial \phi}{\partial x}\right)_{w}\right) \delta \phi_{w} \mathrm{~d} z+\int_{R(t)}^{L}\left(\left(\frac{\partial \eta}{\partial t}+\left(\frac{\partial \phi}{\partial x}\right)_{s} \frac{\partial \eta}{\partial x}-\left(\frac{\partial \phi}{\partial z}\right)_{s}\right) \delta \phi_{s}\right. \\
& \left.\left.-\left(\frac{\partial \phi_{s}}{\partial t}+g \eta+\frac{1}{2}\left(\frac{\partial \phi_{s}}{\partial x}\right)^{2}-\frac{1}{2}\left(\frac{\partial \phi}{\partial z}\right)_{s}^{2}\left(1+\left(\frac{\partial \eta}{\partial x}\right)^{2}\right)\right) \delta \eta\right) \mathrm{d} x\right) \mathrm{d} t
\end{aligned}
$$

Given the arbitrariness of the variations involved in 4.5 , the following dimensional equations of motion emerge

$$
\begin{aligned}
& \nabla^{2} \phi=0 \quad \text { in } \Omega(t), \\
& \frac{\mathrm{d} R}{\mathrm{~d} t}=\frac{\partial \phi}{\partial x} \quad \text { at } S_{W}(t): x=R(t), \\
& \mathbf{n} \cdot \nabla \phi=0 \quad \text { at } S_{B}: z=-H(x) \cup S_{L}: x=L, \\
& \frac{\partial \eta}{\partial t}+\left(\frac{\partial \phi_{s}}{\partial x}\right) \frac{\partial \eta}{\partial x}-\left(\frac{\partial \phi}{\partial z}\right)_{s}\left(1+\left(\frac{\partial \eta}{\partial x}\right)^{2}\right)=0 \quad \text { at } S_{F}(t): z=\eta(x, t), \\
& \frac{\partial \phi_{s}}{\partial t}+\frac{1}{2}\left(\frac{\partial \phi_{s}}{\partial x}\right)^{2}+g \eta-\frac{1}{2}\left(\frac{\partial \phi}{\partial z}\right)_{s}^{2}\left(1+\left(\frac{\partial \eta}{\partial x}\right)^{2}\right)=0 \text { at } S_{F}(t): z=\eta(x, t),
\end{aligned}
$$

viz. the Laplace equation with Neumann boundary conditions (at solid walls) and the free surface equations expressed in terms of the surface potential $\phi_{s}$ and wave height $\eta$. Recall that $\partial\left(\phi_{s}\right) / \partial t \neq(\partial \phi / \partial t)_{s}$, cf. 4.4a.

\subsection{Variational Finite Element Formulation}

\subsubsection{Tessellation}

A point $\mathbf{x} \in \mathbb{R}^{3}$ in the space-time domain $\mathcal{E}$ has coordinates $\mathbf{x}=(t, x, z)$. The flow domain $\Omega(t)$ at time $t$ is defined as $\Omega(t):=\left\{(x, z) \in \mathbb{R}^{2} \mid(t, x, z) \in \mathcal{E}\right\}$. The space-time domain boundary $\partial \mathcal{E}$ consists of the hypersurfaces $\Omega(0):=\{\mathbf{x} \in \partial \mathcal{E} \mid t=0\}, \Omega(T):=$ $\{\mathbf{x} \in \partial \mathcal{E} \mid t=T\}$ and $\mathcal{Q}:=\{\mathbf{x} \in \partial \mathcal{E} \mid 0<t<T\}=: S_{F} \cup S_{W} \cup S_{B} \cup S_{L} \times(0, T)$.

Using the partition $0 \leq \cdots<t^{n}<\cdots \leq t^{N+1}=T$ of the time interval $(0, T)$, we can subdivide the space-time domain $\mathcal{E}$ into space-time slabs $\mathcal{E}^{n}:=\{\mathrm{x} \in \mathcal{E} \mid t \in$ $\left.\left(t^{n}, t^{n+1}\right)\right\}$ with $0 \leq n \leq N$. If the space-time boundary faces that are part of $\mathcal{Q}$ are restricted to the space-time slab $\mathcal{E}^{n}$ then we use the superscript $n$. The remaining boundaries of the space-time slab $\mathcal{E}^{n}$ are $\Omega^{n}=\Omega\left(t^{n}\right)$ and $\Omega^{n+1}=\Omega\left(t^{n+1}\right)$. 
The tessellation $\mathcal{T}_{h}$ is constructed by generating a mesh with quadrilateral elements in the domain $\Omega(t)$, yielding the computational domain $\Omega_{h}(t)$. The spatial elements are denoted as $K_{k_{e}}$, with $k_{e}$ the element index in the tessellation. Due to the free surface and wave maker motion the nodes of each element $K_{k_{e}}^{n}$ at $t=t^{n}$ in domain $\Omega_{h}\left(t^{n}\right)$ will move to a new position at $t=t^{n+1}$, resulting in the spatial element $K_{k_{e}}^{n+1}$ and domain $\Omega_{h}\left(t^{n+1}\right)$. The mesh deformation due to the domain boundary motion is unknown a priori and part of the solution. The geometry of the elements is therefore updated during the solution process.

We will consider structured meshes. Nodes at the free surface and wave maker belong, respectively, to the sets $\mathcal{N}_{F}$ and $\mathcal{N}_{W}$; nodes in the domain $\Omega_{h}$ are collected in the set $\mathcal{N}_{\Omega_{h}}$ and nodes of the domain excluding the free surface nodes in $\bar{\Omega}_{h} \backslash S_{F}$, with $\bar{\Omega}_{h}$ the closure of $\Omega_{h}$, in the set $\mathcal{N}_{\bar{\Omega}_{h}}$. The domain is split in two parts: in $\Omega_{1 h}: R(t)<x<L_{w}$ the nodes in the mesh can move horizontally as well as vertically and in $\Omega_{2 h}: L_{w}<x<L$ with $L_{w}<L$ and $-H(x)<z<\eta(x, t)$ the nodes in mesh move only in the vertical.

\subsubsection{Function spaces and approximations}

The space for two-dimensional basis functions $\tilde{\varphi}$ on $\Omega$ for the potential $\phi$ is $W^{1}(\Omega)=$ $\left\{\tilde{\varphi} \in H^{1}(\Omega)\right\}$ with the standard Sobolev space $H^{1}(\Omega)$. The approximation $\phi_{h}$ to potential $\phi$ is $\phi_{h} \in W_{h}^{k_{p}}=W^{1}(\Omega) \cup X_{h}^{k_{p}}$ with $X_{h}^{k_{p}}$ a finite element space of continuous functions, and including at least polynomials of degree $k_{p}$ on each element $K_{k_{e}}$. The free surface $S_{F}$ is parameterized with coordinate $x \in[R(t), L]$. The associated space for the one-dimensional basis functions $\varphi$ on $x \in[R(t), L]$ for the free surface height $\eta$ and the free surface potential $\phi_{s}$ is $W^{1}([R(t), L])=\left\{\varphi \in H^{1}([R(t), L])\right\}$.

The nodes of the elements are time dependent, making the standard global basis functions also time dependent, as follows. For simplicity, we use quadrilateral elements with the standard two-dimensional piecewise linear and continuous (global) basis functions $\tilde{\varphi}_{j}(x, z, t)$ in space for the "interior" potential $\phi(x, z, t)$. The four local basis functions on a quadrilateral element $K_{k_{e}}$ are

$$
\hat{\psi}_{\alpha}\left(\zeta_{1}, \zeta_{2}\right)=\frac{1}{4}\left(1 \pm \zeta_{1}\right)\left(1 \pm \zeta_{2}\right)
$$

on the reference element $\widehat{K}=\left(\zeta_{1}, \zeta_{2}\right)^{T}=[-1,1]^{2}$ with local node numbers $\alpha=$ $1, \ldots, 4$. Physical coordinates and local reference coordinates are related by a mapping $F_{k_{e}}$ with the same standard shape basis functions

$$
F_{k_{e}}: \widehat{K} \rightarrow K_{k_{e}}:\left(\zeta_{1}, \zeta_{2}\right) \mapsto(x, z):=\left(x_{k_{e}(\alpha)}, z_{k_{e}(\alpha)}\right) \hat{\psi}_{\alpha}\left(\zeta_{1}, \zeta_{2}\right),
$$

with the four vertex coordinates $\left(x_{k_{e}(\alpha)}(t), z_{k_{e}(\alpha)}(t)\right)$ of $K_{k_{e}}$ at time $t$, global node number $j=k_{e}(\alpha)$, in which Einstein's convention of summation over repeated indices (here $\alpha$ ) has been used. Similarly, one-dimensional piecewise linear and continuous basis functions $\varphi_{l}(x, t)$ in space are used at the free surface, for $\eta(x, t)$ and $\phi_{s}(x, t)$. Given 
free-surface element $\bar{K}_{k_{s}}$ and local node number $\beta$, the global free-surface node number $l=k_{s}(\beta)$. The mapping for each free surface face $\bar{K}_{k_{s}}$ to the reference element $\bar{K}=\zeta \in[-1,1]$ reads

$$
\bar{F}_{s}: \bar{K} \rightarrow \bar{K}_{k_{s}}: \zeta \mapsto x=x_{k_{s}(\beta)}(t) \psi_{\beta}(\zeta),
$$

with $x$-coordinates $x_{k_{s}(\beta)}(t)$ of the two surface nodes. The standard two elemental shape functions with $k_{s}$ the global free-surface element index are $\varphi_{k_{s}(\beta)}(x(\zeta, t), t)=\psi_{\beta}(\zeta)=$ $\frac{1}{2}(1 \pm \zeta)$ with local index $\beta=1,2$. When reference coordinates are used the time dependence in the formulation appears in the Jacobian of the mapping (see also Appendix A).

The resulting global finite element approximations are

$$
\begin{aligned}
& \phi_{h}(x, z, t)=\phi_{j}(t) \tilde{\varphi}_{j}(x, z, t), \\
& \phi_{s h}(x, t)=\phi_{k}(t) \varphi_{k}(x, t) \quad \text { and } \quad \eta_{h}(x, t)=\eta_{l}(t) \varphi_{l}(x, t),
\end{aligned}
$$

in which indices $i, j=1, \ldots, N_{n}$ concern all nodes of the mesh and indices $k, l, r, s=$ $1, \ldots, N_{f}$ only the free surface nodes (indices $r, s$ are used later). Summation over repeated indices is adopted. Hereafter, nodes indexed by $i^{\prime}, j^{\prime}$ concern all nodes except the free surface ones. Due to the definition in reference coordinates, it follows that $\left(\tilde{\varphi}_{l}\right)_{s}=\varphi_{l}$, i.e., aligned here by taking $\zeta_{2}=1$ (see Appendices A and B).

Following our restriction to a single-valued free surface and a parameterization of the free surface as $S_{F}:(x, z=\eta(x, t))$, the $x$-coordinate of the free surface nodes is a prescribed time-dependent function $x_{k}(t)$ in the $x$-direction, while the $z$-coordinate is $z=\eta_{h}\left(x_{k}, t\right)$. When there is no wave maker motion, the horizontal node positions $x_{i}$ are fixed in time. A consequence of this restriction is that the movements of the verticallyaligned interior nodes are slaved to the corresponding free surface nodes at the respective $x$-location. The computational mesh in the computational flow domain $\Omega_{1 h}(t)$ is then obtained by moving each node $i_{k}$ according to:

$$
x_{i_{k}}(t)=x_{i_{k}}(0)+\gamma_{x, i_{k}} R(t) \quad \text { and } \quad z_{i_{k}}(t)=z_{i_{k}}(0)+\gamma_{z, i_{k}} \eta\left(x_{k}, t\right),
$$

with the multiplication factors given by $\gamma_{x, i_{k}}:=\left(L_{w}-x_{i_{k}}(0)\right) / L_{w}$ and $\gamma_{z, i_{k}}:=\left(H\left(x_{k}\right)+z_{i_{k}}(0)\right) / H\left(x_{k}\right)$. Nodes $i_{k}$ below the respective free surface node $k$ lie on vertical mesh lines. For the free surface node $k$, we find indeed that $z_{k}=\eta\left(x_{k}, t\right)$ since $z_{k}(0)=0$. For the one at the bottom $z_{i_{k}}(t)=-H\left(x_{k}\right)$ since then $z_{i_{k}}(0)=-H\left(x_{k}\right)$. When the initial free surface at $t=0$ has no elevation, then $z_{k}(0)=0$ for nodes at the free surface. So here we have limited the nodes $x_{i_{k}}(0)$ and $z_{i_{k}}(0)$ to form a structured mesh. In $\Omega_{2 h}$, only the vertical movement in 4.11 is retained with no horizontal node movement. A corresponding sketch of the discretized domain is given in Fig. 4.2 Our finite element approach permits this limitation to be lifted for a general parameterization $S_{F}:\left(x_{s}(t), z_{s}(t)\right)$ given free surface nodes $k$ with positions $\left(x_{s, k}, z_{s, k}\right)$.

The consequence of the mesh movement is therefore that the basis functions in 4.10) depend explicitly on time. Even for the case of unstructured mesh movement, this time 
dependence can generically be expressed as

$$
\tilde{\varphi}_{j}(x, z, t)=\tilde{\varphi}_{j}(x, z ; \boldsymbol{\eta}, R(t)) \quad \text { and } \quad \varphi_{j}(x, t)=\varphi_{j}(x ; R(t)) .
$$

The vector $\boldsymbol{\eta}(t)$ has components $\eta_{r}(t)$, the vector $\tilde{\boldsymbol{\phi}}(t)$ components $\phi_{i^{\prime}}(t)$ at interior nodes, and $\phi$ components $\phi_{l}(t)$ at free surface nodes. Hence, basis functions implicitly depend on time through the time dependence of the variables $\boldsymbol{\eta}$ and explicitly depend on time through the prescribed function $R(t)$ for the piston position. The basis functions on the free surface do therefore not depend on time when $R(t)$ is constant, i.e., in the absence of a wave maker. Hereafter we may not always distinguish these different types of time dependence in the basis functions explicitly.

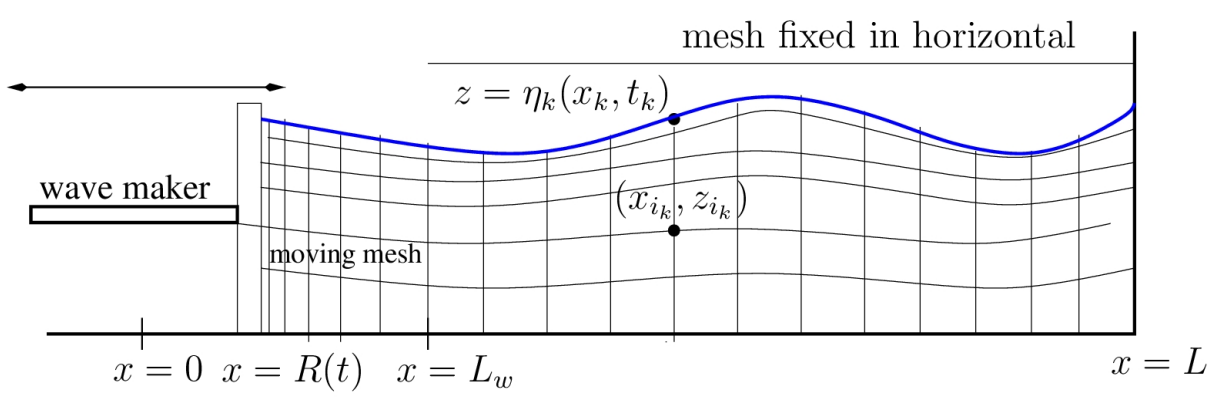

Figure 4.2: A sketch is made of the discrete wave basin with a mesh in the area $R(t)<$ $x<L_{w}$ and $-H(x)<z<\eta(x, t)$ influenced by the piston wave maker at $x=R(t)$ with both horizontal and vertical mesh movement, and an area $L_{w}<x<L$ with only vertical mesh movement.

\subsubsection{Space-plus-time formulation}

We demonstrate how the two key challenges (i) and (ii) mentioned in 4.1 are resolved by first discretizing the variational principle (4.1) in space and subsequently in time. Without any integration by parts or use of Gauss' law, substitution of expansions 4.10) into the variational principle 4.1 yields

$$
\begin{aligned}
0 & =\delta \int_{0}^{T} L(\tilde{\boldsymbol{\phi}}, \boldsymbol{\phi}, \boldsymbol{\eta} ; R(t)) \mathrm{d} t \\
& =\delta \int_{0}^{T}\left(M_{k l} \phi_{k} \frac{\mathrm{d} \eta_{l}}{\mathrm{~d} t}-D_{k l} \phi_{k} \eta_{l}-\frac{1}{2} g M_{k l} \eta_{k} \eta_{l}-\frac{1}{2} A_{i j} \phi_{i} \phi_{j}-W_{m} \phi_{m}\right) \mathrm{d} t,
\end{aligned}
$$


with the following matrices and vectors defined as

$$
\begin{aligned}
& M_{k l}(R(t)):=\int_{R(t)}^{L} \varphi_{k} \varphi_{l} \mathrm{~d} x, \quad D_{k l}(R(t)):=-\int_{R(t)}^{L} \frac{\partial \varphi_{l}}{\partial t} \varphi_{k} \mathrm{~d} x, \\
& A_{i j}(\boldsymbol{\eta}(t), R(t)):=\int_{\Omega_{h}(t)}\left(\nabla \tilde{\varphi}_{i} \cdot \nabla \tilde{\varphi}_{j}\right) \mathrm{d} x \mathrm{~d} z, \\
& W_{m}\left(\eta_{1}(t), R(t)\right):=\left.\int_{-H_{w}(t)}^{\eta_{1}(t)} \frac{\mathrm{d} R}{\mathrm{~d} t} \tilde{\varphi}_{m}\right|_{x=R(t)} \mathrm{d} z,
\end{aligned}
$$

where $\eta_{1}(t)$ is the wave height variable on the wave maker, and index $m$ is used for the nodes on the wave maker. The first node with $m=1$ lies at the free surface and all other nodes on the wave maker carry index $m^{\prime}$. We will also use index $\tilde{m}^{\prime}$ for these interior wave maker nodes. Note that it may be desirable to discretize the bottom boundary also in a piecewise linear manner. Near the wave maker the bottom is, for simplicity, taken to be flat: $H_{w}=H(R(t))$ is constant. These different kinds of time dependence have been explicitly indicated in 4.14 following that dependence in 4.12). The dependence $A_{i j}(\boldsymbol{\eta})$ signifies that $A_{i j}$ in principle depends on all $\eta_{k}$ 's.

Variation of (4.13) while incorporating (4.14) yields

$$
\begin{aligned}
0=\int_{0}^{T} & \left(M_{k l} \frac{\mathrm{d} \eta_{l}}{\mathrm{~d} t}-D_{k l} \eta_{l}-A_{i k} \phi_{i}-W_{1} \delta_{1 k}\right) \delta \phi_{k} \\
& -\left(\frac{\mathrm{d}\left(M_{k l} \phi_{k}\right)}{\mathrm{d} t}+D_{k l} \phi_{k}+g M_{k l} \eta_{k}+\frac{1}{2} \frac{\partial A_{i j}}{\partial \eta_{l}} \phi_{i} \phi_{j}+\frac{\partial W_{m}}{\partial \eta_{1}} \delta_{1 l} \phi_{m}\right) \delta \eta_{l} \\
& \left.-\left(A_{i^{\prime} j^{\prime}} \phi_{i^{\prime}}+A_{l j^{\prime}} \phi_{l}+W_{m^{\prime}} \delta_{m^{\prime} j^{\prime}}\right) \delta \phi_{j^{\prime}}\right) \mathrm{d} t
\end{aligned}
$$

in which the boundary term arising from the integration by parts in time has vanished because we used $\delta \eta_{l}(0)=\delta \eta_{l}(T)=0$ (the first relation says that the variation of the initial condition is zero, while the latter follows similarly by reversing time), and in which $\delta_{m k}$ is the Kronecker delta symbol: it is one when $m=k$ and zero otherwise. The ordinary differential equations are therefore

$$
\begin{array}{r}
\delta \eta_{l}: \frac{\mathrm{d}\left(M_{k l} \phi_{k}\right)}{\mathrm{d} t}+D_{k l} \phi_{k}+g M_{k l} \eta_{k}+\frac{1}{2} \frac{\partial A_{i j}}{\partial \eta_{l}} \phi_{i} \phi_{j}+\frac{\partial W_{m}}{\partial \eta_{1}} \delta_{1 l} \phi_{m}=0 \\
\delta \phi_{k}: M_{k l} \frac{\mathrm{d} \eta_{l}}{\mathrm{~d} t}-D_{k l} \eta_{l}-A_{i k} \phi_{i}-W_{1} \delta_{1 k}=0 \\
\delta \phi_{j^{\prime}}: A_{i^{\prime} j^{\prime}} \phi_{i^{\prime}}+A_{l j^{\prime}} \phi_{l}+W_{m^{\prime}} \delta_{m^{\prime} j^{\prime}}=0
\end{array}
$$


with clearly distinguished variations of surface degrees of freedom arising from the arbitrariness of $\delta \eta_{l}$ and $\delta \phi_{k}$, and interior ones arising from $\delta \phi_{j^{\prime}}$.

The discretized Laplace equation reveals that we can eliminate the interior degrees of freedom indexed by $i^{\prime}, j^{\prime}$ as follows

$$
\phi_{i^{\prime}}=-A_{l j^{\prime}} A_{j^{\prime} i^{\prime}}^{-1} \phi_{l}-W_{m^{\prime}} A_{m^{\prime} i^{\prime}}^{-1}
$$

Substitution of 4.17) into the variational principle (4.13) then leads to Hamiltonian free surface dynamics governed by the following variational principle

$$
\begin{aligned}
0 & =\delta \int_{0}^{T} L(\boldsymbol{\phi}, \boldsymbol{\eta} ; R(t)) \mathrm{d} t \\
& =\delta \int_{0}^{T}\left(M_{k l} \phi_{k} \frac{\mathrm{d} \eta_{l}}{\mathrm{~d} t}-\frac{1}{2} g M_{k l} \eta_{k} \eta_{l}-\frac{1}{2} B_{k l} \phi_{k} \phi_{l}\right. \\
& \left.-D_{k l} \phi_{k} \eta_{l}-C_{l} \phi_{l}-F_{w}\right) \mathrm{d} t,
\end{aligned}
$$

in which the Schur complement

$$
B_{k l}(\boldsymbol{\eta}, R(t))=\left(A_{k l}-A_{k i^{\prime}} A_{i^{\prime} j^{\prime}}^{-1} A_{j^{\prime} l}\right)
$$

of the full matrix $A_{i j}$ emerges as a consequence of this elimination of the interior degrees of freedom, a vector $C_{l}$ and a function $F_{w}$, i.e.,

$$
C_{l}(\boldsymbol{\eta}, R(t))=W_{1} \delta_{1 l}-A_{l j^{\prime}} A_{j^{\prime} m^{\prime}}^{-1} W_{m^{\prime}} \text { and } F_{w}(t)=-\frac{1}{2} W_{\tilde{m}^{\prime}} A_{\tilde{m}^{\prime} m^{\prime}}^{-1} W_{m^{\prime}}
$$

The notation $B_{k l}(\boldsymbol{\eta})$ means that $B_{k l}$ depends in principle on all surface degrees of freedom $\boldsymbol{\eta}$.

Variational principle 4.18 in essence captures a discrete boundary element representation of the dynamics. One can verify that the dynamics 4.16, in which the interior degrees of freedom $\phi_{i^{\prime}}$ or $\phi_{j^{\prime}}$ have been eliminated using (4.17), follows directly from this free-surface variational principle 4.18 . We never calculate the matrix inverse $A_{i^{\prime} j^{\prime}}^{-1}$ explicitly. This inverse is only used to derive the appropriate time stepping scheme in the next step. In the end, we "unfold" the algebraic system again to avoid a direct calculation of this inverse matrix because that is computationally expensive. It is large and depends implicitly and explicitly on time.

The variational principle 4.18 is a canonical Hamiltonian system

$$
0=\delta \int_{0}^{T}\left(p_{l} \frac{\mathrm{d} \eta_{l}}{\mathrm{~d} t}-H(\mathbf{p}, \boldsymbol{\eta} ; R(t))\right) \mathrm{d} t
$$


with conjugate variables $\left\{\mathbf{p}_{l}, \boldsymbol{\eta}_{l}\right\}=\left\{p_{l}=M_{k l} \phi_{k}, \eta_{l}\right\}$ and extended (Hamiltonian) function $H=H_{h}+H_{n a}$ split into the following Hamiltonian, $H_{h}$, and wave maker part, $H_{n a}$ (non-autonomous),

$$
H_{h} \equiv \frac{1}{2} g M_{k l} \eta_{k} \eta_{l}+\frac{1}{2} B_{k l} \phi_{k} \phi_{l} \quad \text { and } \quad H_{n a} \equiv D_{k l} \phi_{k} \eta_{l}+C_{l} \phi_{l}+F_{w} .
$$

Without wave maker $H_{h}$ is time independent and $H_{n a}$ disappears. A standard yet semiimplicit and symplectic finite-difference Störmer-Verlet time integrator [64] would then suffice to discretize 4.18) for $R(t)=0$ (or 4.19) with $H_{n a}=0$ ).

The explicit time dependence that makes 4.18 a non-autonomous variational principle stimulated us to develop a discontinuous Galerkin finite element approach in time. In each time slab the DG basis functions $\psi_{t}(t)$ are continuous and the associated space is $V_{h}^{k_{t}}=\left\{\psi_{t} \in \mathcal{P}_{k_{t}}\left(t \in\left(t^{n}, t^{n+1}\right)\right)\right\}$ with $\mathcal{P}_{k_{t}}$ polynomials of degree $k_{t}$. We will restrict attention to piecewise linear polynomials $\psi_{t}(t)$, i.e., $k_{t}=1$. We choose the following linear expansions within each time slab $t \in\left(t^{n}, t^{n+1}=t^{n}+\Delta t^{n}\right)$ :

$$
\begin{aligned}
\eta_{l}(t) & =\eta_{l}^{n,+}\left(\frac{t^{n+1}-t}{\Delta t^{n}}\right)+\eta_{l}^{n+1,-}\left(\frac{t-t^{n}}{\Delta t^{n}}\right) \text { and } \\
\phi_{k}(t) & =\phi_{k}^{n+1 / 2} \frac{2\left(t-t_{n}\right)}{\Delta t^{n}}+\phi_{k}^{n,+} \frac{\left(t^{n}+t^{n+1}-2 t\right)}{\Delta t^{n}}
\end{aligned}
$$

where $\eta_{l}^{n, \pm}=\lim _{\epsilon \downarrow 0} \eta_{l}\left(t^{n} \pm \epsilon\right), \phi_{k}^{n,+}=\lim _{\epsilon \downarrow 0} \phi_{k}\left(t^{n}+\epsilon\right)$ and $\phi_{k}^{n+1 / 2}=\phi_{k}\left(t=t^{n+1 / 2}\right)$. (For symplectic schemes the time step needs to be fixed, such that $\Delta t^{n}=\Delta t$, also in the simulations presented later.) A sketch of these temporal basis functions is given in Fig. 4.3 These expansions are continuous within the time slab but discontinuous across. Hence, we have to define the delta function arising from the time derivative in the term $M_{k l} \phi_{k} \mathrm{~d} \eta_{l} / \mathrm{d} t$ in 4.18 . The contribution of this term can be posited by appealing to the theory of nonconservative products (for hyperbolic systems [34, 125, 126] or quasi-linear equations [147]), and it can be derived (e.g., [52]). Under a specific choice of coefficients, which is discussed in Chapter 3 , at node $t^{n+1}$ an outcome is the contribution:

$$
M_{k l} \phi_{k} \frac{\mathrm{d} \eta_{l}}{\mathrm{~d} t} \approx M_{k l}^{n+1,+} \phi_{k}^{n+1,+}\left(\eta_{l}^{n+1,+}-\eta_{l}^{n+1,-}\right),
$$

i.e., $M_{k l} \phi_{k}$ is evaluated on the future side of node $t^{n+1}$ and a jump in $\eta$ across $t^{n+1}$ emerges. When $M_{k l}$ is considered continuous in time, i.e., for continuous $R(t)$, then $M_{k l}^{n+1,+}=M_{k l}^{n+1}$. We will take $R(t)$ continuous.

The other terms in the time integral of (4.18) are approximated over each time slab such that they are second order in time. The resulting integrals are then approximated by 

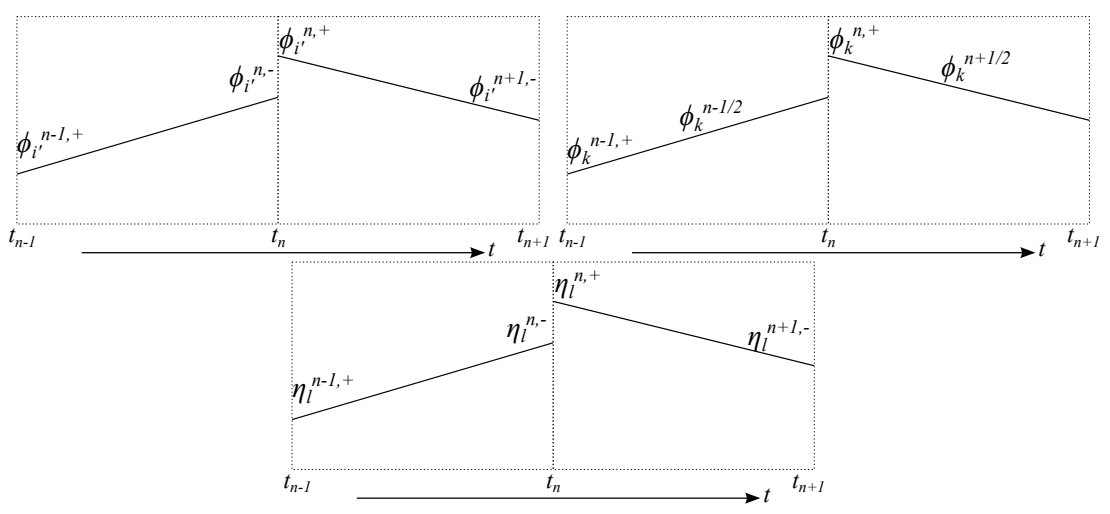

Figure 4.3: Piecewise linear approximation in time of the potential $\phi$, free surface potential $\phi_{s}$ and wave height $\eta$.

the following quadrature rules:

$$
\begin{aligned}
& \int_{t_{n}}^{t_{n+1}} M_{k l} \phi_{k} \frac{\mathrm{d} \eta_{l}}{\mathrm{~d} t} \mathrm{~d} t \approx M_{k l}^{n+1 / 2} \phi_{k}^{n+1 / 2}\left(\eta_{l}^{n+1,-}-\eta_{l}^{n,+}\right) ; \\
& \int_{t_{n}}^{t_{n+1}} D_{k l} \phi_{k} \eta_{l} \mathrm{~d} t \approx \frac{1}{2} \Delta t^{n} D_{k l}^{n+1 / 2} \phi_{k}^{n+1 / 2}\left(\eta_{l}^{n,+}+\eta_{l}^{n+1,-}\right) ; \\
& \int_{t_{n}}^{t_{n+1}} \frac{1}{2} M_{k l} \eta_{k} \eta_{l} \mathrm{~d} t \approx \frac{1}{4} \Delta t^{n} M_{k l}^{n+1 / 2}\left(\eta_{k}^{n,+} \eta_{l}^{n,+}+\eta_{k}^{n+1,-} \eta_{l}^{n+1,-}\right) ;
\end{aligned}
$$

and,

$$
\begin{aligned}
& \int_{t_{n}}^{t_{n+1}} \frac{1}{2} B_{k l} \phi_{k} \phi_{l} \mathrm{~d} t \approx \\
& \quad \frac{1}{4} \Delta t^{n}\left(B_{k l}^{n+1 / 2}\left(\boldsymbol{\eta}^{n,+}\right)+B_{k l}^{n+1 / 2}\left(\boldsymbol{\eta}^{n+1,-}\right)\right) \phi_{k}^{n+1 / 2} \phi_{l}^{n+1 / 2}, \\
& \int_{t_{n}}^{t_{n+1}} C_{l} \phi_{l} \mathrm{~d} t \approx \frac{1}{2} \Delta t^{n}\left(C_{l}^{n+1 / 2}\left(\boldsymbol{\eta}^{n,+}\right)+C_{l}^{n+1 / 2}\left(\boldsymbol{\eta}^{n+1,-}\right)\right) \phi_{l}^{n+1 / 2} .
\end{aligned}
$$

In the first integral, we either approximate $M_{k l}$ at the mid-time level, or we can view it as an exact evaluation of the entire conjugate variable expanded in a linear way in time. 
The remaining integrals are approximated using a mid-point rule with $\phi_{k}$, trapezoidal rule with $\eta_{l}$ and time evaluated at $t=t^{n+1 / 2}$. Here, $C_{l}^{n+1 / 2}\left(\boldsymbol{\eta}^{n,+}\right)=C_{l}\left(\boldsymbol{\eta}^{n,+}, R\left(t^{n+1 / 2}\right)\right)$, etc. The time derivative of $\varphi$ in $D_{k l}$ leads via the chain rule to a partial derivative with respect to $R(t)$ times $\mathrm{d} R / \mathrm{d} t$. The latter is either found explicitly or $R(t)$ is approximated and then all expressions are evaluated at $t^{n+1 / 2}$. Hence, by combining the resulting approximations derived in (4.22) and (4.23) the algebraic, space-time discrete variational principle at the free surface in essence becomes

$$
\begin{aligned}
0= & \delta \sum_{n=0}^{N} L\left[\boldsymbol{\phi}^{n,+}, \boldsymbol{\phi}^{n+1 / 2}, \boldsymbol{\eta}^{n,+}, \boldsymbol{\eta}^{n+1,-}\right] \\
= & \delta \sum_{n=0}^{N} M_{k l}^{n+1 / 2} \phi_{k}^{n+1 / 2}\left(\eta_{l}^{n+1,-}-\eta_{l}^{n,+}\right) \\
& -\frac{\Delta t^{n}}{2}\left(H^{n+1 / 2}\left(\boldsymbol{\phi}^{n+1 / 2}, \boldsymbol{\eta}^{n,+}\right)+H^{n+1 / 2}\left(\boldsymbol{\phi}^{n+1 / 2}, \boldsymbol{\eta}^{n+1,-}\right)\right) \\
& +\delta \sum_{n=-1}^{N} M_{k l}^{n+1} \phi_{k}^{n+1,+}\left(\eta_{l}^{n+1,+}-\eta_{l}^{n+1,-}\right),
\end{aligned}
$$

with $H^{n+1 / 2}\left(\boldsymbol{\phi}^{n+1 / 2}, \boldsymbol{\eta}^{n+1,-}\right)=H\left(\boldsymbol{\phi}^{n+1 / 2}, \boldsymbol{\eta}^{n+1,-}, t^{n+1 / 2}\right)$.

Variation of (4.24) with respect to the independent variables indicated yields an extension of the symplectic Störmer-Verlet time integration scheme

$$
\begin{aligned}
\delta \phi_{k}^{n+1,+}: & \eta_{l}^{n+1,+}=\eta_{l}^{n+1,-} \\
\delta \eta_{l}^{n,+}: & M_{k l}^{n+1 / 2} \phi_{k}^{n+1 / 2}=M_{k l}^{n} \phi_{k}^{n,+}-\frac{1}{2} \Delta t^{n} \frac{\partial H^{n+1 / 2}\left(\phi^{n+1 / 2}, \boldsymbol{\eta}^{n,+}\right)}{\partial \eta_{l}^{n,+}} \\
\delta \phi_{k}^{n+1 / 2}: & M_{k l}^{n+1 / 2} \eta_{l}^{n+1,-}=M_{k l}^{n+1 / 2} \eta_{l}^{n,+} \\
+ & \frac{1}{2} \Delta t^{n}\left(\frac{\partial H^{n+1 / 2}\left(\phi^{n+1 / 2}, \boldsymbol{\eta}^{n,+}\right)}{\partial \phi_{k}^{n+1 / 2}}+\frac{\partial H^{n+1 / 2}\left(\phi^{n+1 / 2}, \boldsymbol{\eta}^{n+1,-}\right)}{\partial \phi_{k}^{n+1 / 2}}\right) \\
\delta \eta_{l}^{n+1,-}: & M_{k l}^{n+1} \phi_{k}^{n+1,+}=M_{k l}^{n+1 / 2} \phi_{k}^{n+1 / 2}-\frac{1}{2} \Delta t^{n} \frac{\partial H^{n+1 / 2}\left(\phi^{n+1 / 2}, \boldsymbol{\eta}^{n+1,-}\right)}{\partial \eta_{l}^{n+1,-}} .
\end{aligned}
$$

Both the choice of the numerical flux (or path) and the quadratures used above were guided by our intention to derive the classic Störmer-Verlet scheme in the absence of explicit time dependence, i.e., here when $R(t)=0$.

Finally, the inverse of the matrix $A_{i^{\prime} j^{\prime}}$ in the non-constant matrix $B_{k l}$ and the nonconstant vector $C_{l}$ can be avoided by unfolding the free surface dynamics again to include 
the interior degrees of freedom. When we use the notations

$$
\begin{aligned}
\phi_{i^{\prime}}^{n,+} & =-A_{l j^{\prime}}^{n+1 / 2}\left(\boldsymbol{\eta}^{n,+}\right) A_{j^{\prime} i^{\prime}}^{-1}\left(\boldsymbol{\eta}^{n,+}, t^{n+1 / 2}\right) \phi_{l}^{n+1 / 2} \\
\phi_{i^{\prime}}^{n+1,-} & =-A_{l j^{\prime}}^{n+1 / 2}\left(\boldsymbol{\eta}^{n+1,-}\right) A_{j^{\prime} i^{\prime}}^{-1}\left(\boldsymbol{\eta}^{n+1,-}, t^{n+1 / 2}\right) \phi_{l}^{n+1 / 2}
\end{aligned}
$$

in the Schur complement approximation (4.23d, we get

$$
\begin{aligned}
\int_{t_{n}}^{t_{n+1}} B_{k l} \phi_{k} \phi_{l} \mathrm{~d} t \approx \\
\quad \frac{1}{2} \Delta t^{n}\left(A_{k l}^{n+1 / 2}\left(\boldsymbol{\eta}^{n,+}\right) \phi_{k}^{n+1 / 2} \phi_{l}^{n+1 / 2}+A_{i^{\prime} l}^{n+1 / 2}\left(\boldsymbol{\eta}^{n,+}\right) \phi_{i^{\prime}}^{n,+} \phi_{l}^{n+1 / 2}\right. \\
\left.\quad+A_{k l}^{n+1 / 2}\left(\boldsymbol{\eta}^{n+1,-}\right) \phi_{k}^{n+1 / 2} \phi_{l}^{n+1 / 2}+A_{i^{\prime} l}^{n+1 / 2}\left(\boldsymbol{\eta}^{n+1,-}\right) \phi_{i^{\prime}}^{n+1,-} \phi_{l}^{n+1 / 2}\right) .
\end{aligned}
$$

Hence, it seems that the finite element expansion for the interior degrees of freedom is

$$
\phi_{i^{\prime}}=\phi_{i^{\prime}}^{n,+}\left(\frac{t^{n+1}-t}{\Delta t^{n}}\right)+\phi_{i^{\prime}}^{n+1,-}\left(\frac{t-t^{n}}{\Delta t^{n}}\right)
$$

with $\phi_{i^{\prime}}^{n, \pm}=\lim _{\epsilon \downarrow 0} \phi_{i^{\prime}}\left(t^{n} \pm \epsilon\right)$.

The integral of the kinetic energy $K=A_{i j} \phi_{i} \phi_{j} / 2$ in the standard Hamiltonian $H_{h}=$ $K+P=A_{i j} \phi_{i} \phi_{j} / 2+g M_{k l} \eta_{k} \eta_{l} / 2$ can be rewritten as

$$
\begin{aligned}
& \sum_{n=0}^{N} \frac{1}{2} \Delta t^{n}\left(K\left(\tilde{\boldsymbol{\phi}}^{n,+}, \boldsymbol{\phi}^{n+1 / 2}, \boldsymbol{\eta}^{n,+}, t^{n+1 / 2}\right)+K\left(\tilde{\boldsymbol{\phi}}^{n+1,-}, \boldsymbol{\phi}^{n+1 / 2}, \boldsymbol{\eta}^{n+1,-}, t^{n+1 / 2}\right)\right) \equiv \\
& \sum_{n=0}^{N} \frac{\Delta t^{n}}{4}\left(A_{k l}^{n+1 / 2}\left(\boldsymbol{\eta}^{n,+}\right) \phi_{k}^{n+1 / 2} \phi_{l}^{n+1 / 2}+A_{k l}^{n+1 / 2}\left(\boldsymbol{\eta}^{n+1,-}\right) \phi_{k}^{n+1 / 2} \phi_{l}^{n+1 / 2}\right. \\
& +A_{k j^{\prime}}^{n+1 / 2}\left(\boldsymbol{\eta}^{n,+}\right) \phi_{k}^{n+1 / 2} \phi_{j^{\prime}}^{n++}+A_{k j^{\prime}}^{n+1 / 2}\left(\boldsymbol{\eta}^{n+1,-}\right) \phi_{k}^{n+1 / 2} \phi_{j^{\prime}}^{n+1,-} \\
& +A_{i^{\prime} l}^{n+1 / 2}\left(\boldsymbol{\eta}^{n,+}\right) \phi_{i^{\prime}}^{n,+} \phi_{l}^{n+1 / 2}+A_{i^{\prime} l}^{n+1 / 2}\left(\boldsymbol{\eta}^{n+1,-}\right) \phi_{i^{\prime}}^{n+1,-} \phi_{l}^{n+1 / 2} \\
& \left.+A_{i^{\prime} j^{\prime}}^{n+1 / 2}\left(\boldsymbol{\eta}^{n,+}\right) \phi_{i^{\prime}}^{n,+} \phi_{j^{\prime}}^{n,+}+A_{i^{\prime} j^{\prime}}^{n+1 / 2}\left(\boldsymbol{\eta}^{n+1,-}\right) \phi_{i^{\prime}}^{n+1,-} \phi_{j^{\prime}}^{n+1,-}\right)
\end{aligned}
$$

after some algebraic manipulations. A similar unfolding can be done for the wave maker term involving $C_{l}$ but is not required.

Analogously, we now see that the time integral of the wave maker contribution in 
4.13) is approximated as

$$
\begin{aligned}
& \int_{0}^{T} W_{m} \phi_{m} \mathrm{~d} t \cong \\
& \sum_{n=0}^{N} \frac{\Delta t^{n}}{2}\left(W_{q}^{n+1 / 2}\left(\eta_{q}^{n,+}\right) \phi_{q}^{n+1 / 2}+W_{q}^{n+1 / 2}\left(\eta_{q}^{n+1,-}\right) \phi_{q}^{n+1 / 2}\right. \\
& \\
& \left.+W_{m^{\prime}}^{n+1 / 2}\left(\eta_{q}^{n,+}\right) \phi_{m^{\prime}}^{n,+}+W_{m^{\prime}}^{n+1 / 2}\left(\eta_{q}^{n+1,-}\right) \phi_{m^{\prime}}^{n+1,-}\right),
\end{aligned}
$$

with interior wave maker nodes $m^{\prime}$, the wave maker node $q=1$ at the free surface and $W_{m^{\prime}}^{n+1 / 2}\left(\eta_{q}^{n,+}\right)=W_{m^{\prime}}\left(\eta_{q}^{n,+}, t^{n+1 / 2}\right)$, etc. The vertices on the wave maker are thus evaluated at time $t^{n+1 / 2}$.

By using approximations $4.23(\mathrm{a}, \mathrm{b}, \mathrm{c})$ for the first three free surface and potential energy terms in 4.13 and approximations 4.29 for the kinetic energy and 4.30 for the wave maker terms in 4.13, the space discrete principle 4.13) becomes the following space-time discrete variational principle

$$
\begin{aligned}
0=\delta & L\left[\tilde{\boldsymbol{\phi}}^{n,+}, \tilde{\boldsymbol{\phi}}^{n+1,-}, \boldsymbol{\phi}^{n,+}, \boldsymbol{\phi}^{n+1 / 2}, \boldsymbol{\eta}^{n,+}, \boldsymbol{\eta}^{n+1,-}\right] \\
=\delta & \sum_{n=0}^{N} M_{k l}^{n+1 / 2} \phi_{l}^{n+1 / 2}\left(\eta_{k}^{n+1,-}-\eta_{k}^{n,+}\right)-\frac{1}{2} \Delta t^{n} D_{k l}^{n+1 / 2} \phi_{l}^{n+1 / 2}\left(\eta_{k}^{n,+}+\eta_{k}^{n+1,-}\right) \\
& \quad-\frac{1}{2} \Delta t^{n}\left(H_{h}^{n+1 / 2}\left(\tilde{\boldsymbol{\phi}}^{n,+}, \boldsymbol{\phi}^{n+1 / 2}, \boldsymbol{\eta}^{n,+}\right)+H_{h}^{n+1 / 2}\left(\tilde{\boldsymbol{\phi}}^{n+1,-}, \boldsymbol{\phi}^{n+1 / 2}, \boldsymbol{\eta}^{n+1,-}\right)\right) \\
& -\frac{1}{2} \Delta t^{n}\left(\left(W_{q}^{n+1 / 2}\left(\eta_{q}^{n,+}\right)+W_{q}^{n+1 / 2}\left(\eta_{q}^{n+1,-}\right)\right) \phi_{q}^{n+1 / 2}\right. \\
& \left.\quad+W_{m^{\prime}}^{n+1 / 2}\left(\eta_{q}^{n,+}\right) \phi_{m^{\prime}}^{n+,}+W_{m^{\prime}}^{n+1 / 2}\left(\eta_{q}^{n+1,-}\right) \phi_{m^{\prime}}^{n+1,-}\right) \\
& +\sum_{n=-1}^{N} M_{k l}^{n+1} \phi_{l}^{n+1,+}\left(\eta_{k}^{n+1,+}-\eta_{k}^{n+1,-}\right),
\end{aligned}
$$

with indices $k, l \in \mathcal{N}_{F}, m^{\prime} \in \mathcal{N}_{W} \backslash \mathcal{N}_{F}, i^{\prime}, j^{\prime} \in \mathcal{N}_{\bar{\Omega}_{h}}$ and $q \in \mathcal{N}_{F} \cap \mathcal{N}_{W}$. It consists of sums of discretized integrals and jump terms over and across the space-time slabs $\mathcal{E}^{n}$.

\subsubsection{Dynamics}

Taking variations of 4.31 over the independent variables $\phi_{l}^{n+1 / 2}, \phi_{l}^{n,+}, \phi_{i^{\prime}}^{n,+}, \phi_{i^{\prime}}^{n+1,-}$ ,$\eta_{k}^{n,+}$ and $\eta_{k}^{n+1,-}$ yields an algebraic system for these potential and wave height coeffi- 
cients:

$$
\begin{aligned}
\delta \phi_{l}^{n+1,+} & : M_{k l}^{n+1} \eta_{k}^{n+1,+}=M_{k l}^{n+1} \eta_{k}^{n+1,-}, \\
\delta \phi_{i^{\prime}}^{n,+} & : \frac{\partial H_{h}^{n+1 / 2}\left(\tilde{\boldsymbol{\phi}}^{n,+}, \boldsymbol{\phi}^{n+1 / 2}, \boldsymbol{\eta}^{n,+}\right)}{\partial \phi_{i^{\prime}}^{n,+}}=-W_{m^{\prime}}^{n+1 / 2}\left(\eta_{q}^{n,+}\right) \delta_{m^{\prime} i^{\prime}}, \\
\delta \eta_{k}^{n,+}: & \left(M_{k l}^{n+1 / 2}+\frac{1}{2} \Delta t^{n} D_{k l}^{n+1 / 2}\right) \phi_{l}^{n+1 / 2}=M_{k l}^{n} \phi_{l}^{n,+} \\
& -\frac{1}{2} \Delta t^{n}\left(\frac{\partial H_{h}^{n+1 / 2}\left(\tilde{\boldsymbol{\phi}}^{n,+}, \boldsymbol{\phi}^{n+1 / 2}, \boldsymbol{\eta}^{n,+}\right)}{\partial \eta_{k}^{n,+}}+\frac{\partial W_{q}^{n+1 / 2}\left(\eta_{q}^{n,+}\right)}{\partial \eta_{q}^{n,+}} \phi_{q}^{n+1 / 2} \delta_{k q}\right. \\
& +\frac{\partial W_{m^{\prime}}^{n+1 / 2}\left(\eta_{q}^{n,+}\right)}{\left.\partial \eta_{q}^{n,+} \phi_{m^{\prime}}^{n,+} \delta_{k q}\right),} \\
\delta \phi_{l}^{n+1 / 2} & :\left(M_{k l}^{n+1 / 2}-\frac{1}{2} \Delta t^{n} D_{k l}^{n+1 / 2}\right) \eta_{k}^{n+1,-}=\left(M_{k l}^{n+1 / 2}+\frac{1}{2} \Delta t^{n} D_{k l}^{n+1 / 2}\right) \eta_{k}^{n,+} \\
& +\frac{1}{2} \Delta t^{n}\left(\frac{\partial H_{h}^{n+1 / 2}\left(\tilde{\boldsymbol{\phi}}^{n,+}, \boldsymbol{\phi}^{n+1 / 2}, \boldsymbol{\eta}^{n,+}\right)}{\partial \phi_{l}^{n+1 / 2}}+\frac{\partial H_{h}^{n+1 / 2}\left(\tilde{\boldsymbol{\phi}}^{n+1,-}, \boldsymbol{\phi}^{n+1 / 2}, \boldsymbol{\eta}^{n+1,-}\right)}{\partial \phi_{l}^{n+1 / 2}}\right) \\
& +\frac{1}{2} \Delta t^{n}\left(W_{q}^{n+1 / 2}\left(\eta_{q}^{n,+}\right)+W_{q}^{n+1 / 2}\left(\eta_{q}^{n+1,-}\right)\right) \delta_{q l}, \\
\delta \phi_{i^{\prime}}^{n+1,-} & : \frac{\partial H_{h}^{n+1 / 2}\left(\tilde{\boldsymbol{\phi}}^{n+1,-}, \boldsymbol{\phi}^{n+1 / 2}, \boldsymbol{\eta}^{n+1,-}\right)}{\partial \phi_{i^{\prime}}^{n+1,-}}=-W_{m^{\prime}}^{n+1 / 2}\left(\eta_{q}^{n+1,-}\right) \delta_{m^{\prime} i^{\prime}}, \quad(4.32 \mathrm{e})
\end{aligned}
$$

The variations of the potential energy are straightforward:

$$
\delta\left(\frac{1}{2} g M_{k l}^{n+1 / 2} \eta_{k}^{n_{p}} \eta_{l}^{n_{p}}\right)=g M_{k l}^{n+1 / 2} \eta_{k}^{n_{p}} \delta \eta_{l}^{n_{p}},
$$

with $n_{p}=n,+$ or $n_{p}=n+1,-$. The variations of the kinetic energy with respect to the 
velocity potential include both interior variations $\phi_{j^{\prime}}$ and free surface ones $\phi_{l}$, yielding

$$
\begin{aligned}
\delta_{\phi} K\left(\tilde{\boldsymbol{\phi}}^{n_{p}}, \boldsymbol{\phi}^{n+1 / 2}, \boldsymbol{\eta}^{n_{p}}, t^{n+1 / 2}\right) & \\
= & \left(A_{l j^{\prime}}^{n+1 / 2}\left(\boldsymbol{\eta}^{n_{p}}\right) \phi_{l}^{n+1 / 2}+A_{i^{\prime} j^{\prime}}^{n+1 / 2}\left(\boldsymbol{\eta}^{n_{p}}\right) \phi_{i^{\prime}}^{n_{p}}\right) \delta \phi_{j^{\prime}}^{n_{p}} \\
& \quad+\left(A_{l k}^{n+1 / 2}\left(\boldsymbol{\eta}^{n_{p}}\right) \phi_{l}^{n+1 / 2}+A_{i^{\prime} k}^{n+1 / 2}\left(\boldsymbol{\eta}^{n_{p}}\right) \phi_{i^{\prime}}^{n_{p}}\right) \delta \phi_{k}^{n+1 / 2} .
\end{aligned}
$$

Variations of the kinetic energy with respect to the free surface elevation $\eta_{l}$ are more complicated to compute since the nodal coordinates $\left(x_{i}, z_{i}\right)$ in the mesh change every time step due free surface and piston wave maker movements as described in the 4.7.2 By introducing

$$
C_{l i j}^{n+1 / 2}\left(\boldsymbol{\eta}^{n_{p}}\right)=\frac{\partial A_{i j}^{n+1 / 2}\left(\boldsymbol{\eta}^{n_{p}}\right)}{\partial \eta_{l}^{n_{p}}},
$$

we can compute the variations of the kinetic energy with respect to the free surface elevation

$$
\begin{aligned}
\delta_{\eta} K\left(\tilde{\boldsymbol{\phi}}^{n_{p}}\right. & \left., \boldsymbol{\phi}^{n+1 / 2} ; \boldsymbol{\eta}^{n_{p}}, t^{n+1 / 2}\right) \\
& =\frac{1}{2}\left(C_{r i^{\prime} j^{\prime}}^{n+1 / 2}\left(\boldsymbol{\eta}^{n_{p}}\right) \phi_{i^{\prime}}^{n_{p}} \phi_{j^{\prime}}^{n_{p}}+C_{r l j^{\prime}}^{n+1 / 2}\left(\boldsymbol{\eta}^{n_{p}}\right) \phi_{l}^{n+1 / 2} \phi_{j^{\prime}}^{n_{p}}\right. \\
& \left.+C_{r i^{\prime} k}^{n+1 / 2}\left(\boldsymbol{\eta}^{n_{p}}\right) \phi_{i^{\prime}}^{n_{p}} \phi_{k}^{n+1 / 2}+C_{r l k}^{n+1 / 2}\left(\boldsymbol{\eta}^{n_{p}}\right) \phi_{l}^{n+1 / 2} \phi_{k}^{n+1 / 2}\right) \delta \eta_{r}^{n_{p}} .
\end{aligned}
$$

Finally, after combining all contributions, the variations of the Hamiltonian can be written as

$$
\begin{aligned}
& \delta H_{h}^{n+1 / 2}\left(\tilde{\boldsymbol{\phi}}^{n_{p}}, \boldsymbol{\phi}^{n+1 / 2}, \boldsymbol{\eta}^{n_{p}}\right) \\
&=\left(A_{l j^{\prime}}^{n+1 / 2}\left(\boldsymbol{\eta}^{n_{p}}\right) \phi_{l}^{n+1 / 2}+A_{i^{\prime} j^{\prime}}^{n+1 / 2}\left(\boldsymbol{\eta}^{n_{p}}\right) \phi_{i^{\prime}}^{n_{p}}\right) \delta \phi_{j^{\prime}}^{n_{p}} \\
& \quad+\left(A_{l k}^{n+1 / 2}\left(\boldsymbol{\eta}^{n_{p}}\right) \phi_{l}^{n+1 / 2}+A_{i^{\prime} k}^{n+1 / 2}\left(\boldsymbol{\eta}^{n_{p}}\right) \phi_{i^{\prime}}^{n_{p}}\right) \delta \phi_{k}^{n+1 / 2} \\
&+\left(\frac { 1 } { 2 } \left(C_{r i^{\prime} j^{\prime}}^{n+1 / 2}\left(\boldsymbol{\eta}^{n_{p}}\right) \phi_{i^{\prime}}^{n_{p}} \phi_{j^{\prime}}^{n_{p}}+C_{r l j^{\prime}}^{n+1 / 2}\left(\boldsymbol{\eta}^{n_{p}}\right) \phi_{l}^{n+1 / 2} \phi_{j^{\prime}}^{n_{p}}\right.\right. \\
&\left.+C_{r i^{\prime} k}^{n+1 / 2}\left(\boldsymbol{\eta}^{n_{p}}\right) \phi_{i^{\prime}}^{n_{p}} \phi_{k}^{n+1 / 2}+C_{r l k}^{n+1 / 2}\left(\boldsymbol{\eta}^{n_{p}}\right) \phi_{l}^{n+1 / 2} \phi_{k}^{n+1 / 2}\right) \\
&+\left.g M_{k r}^{n+1 / 2} \eta_{k}^{n_{p}}\right) \delta \eta_{r}^{n_{p}} .
\end{aligned}
$$


Likewise, variations of the wave maker contribution yield

$$
\begin{aligned}
& \delta\left(W_{q}^{n+1 / 2}\left(\eta_{q}^{n_{p}}\right) \phi_{q}^{n+1 / 2}+W_{m^{\prime}}^{n+1 / 2}\left(\eta_{q}^{n_{p}}\right) \phi_{m^{\prime}}^{n_{p}}\right) \\
& =\frac{\partial W_{q}^{n+1 / 2}\left(\eta_{q}^{n_{p}}\right)}{\partial \eta_{q}^{n_{p}}} \phi_{q}^{n+1 / 2} \delta \eta_{q}^{n_{p}}+\frac{\partial W_{m^{\prime}}^{n+1 / 2}\left(\eta_{q}^{n_{p}}\right)}{\partial \eta_{q}^{n_{p}}} \phi_{m^{\prime}}^{n_{p}} \delta \eta_{q}^{n_{p}} \\
& +W_{q}^{n+1 / 2}\left(\eta_{q}^{n_{p}}\right) \delta \phi_{q}^{n+1 / 2}+W_{m^{\prime}}^{n+1 / 2}\left(\eta_{q}^{n_{p}}\right) \delta \phi_{m^{\prime}}^{n_{p}} \\
& =E_{q}^{n+1 / 2}\left(\eta_{q}^{n_{p}}\right) \phi_{q}^{n+1 / 2} \delta \eta_{q}^{n_{p}}+E_{m^{\prime}}^{n+1 / 2}\left(\eta_{q}^{n_{p}}\right) \phi_{m^{\prime}}^{n_{p}} \delta \eta_{q}^{n_{p}} \\
& +W_{q}^{n+1 / 2}\left(\eta_{q}^{n_{p}}\right) \delta \phi_{q}^{n+1 / 2}+W_{m^{\prime}}^{n+1 / 2}\left(\eta_{q}^{n_{p}}\right) \delta \phi_{m^{\prime}}^{n_{p}}
\end{aligned}
$$

with $E_{m}^{n+1 / 2}\left(\eta_{q}^{n_{p}}\right):=\partial W_{m}^{n+1 / 2}\left(\eta_{q}^{n_{p}}\right) / \partial \eta_{q}^{n_{p}}$. As we can see in $\left.4.32 \mathrm{~b}, \mathrm{e}\right)$, there is no dynamical equation for the interior velocity potential $\phi_{i^{\prime}}$ at the nodes in the domain. For convenience' sake, we rename the interior potentials as $\phi_{i^{\prime}}^{n}:=\phi_{i^{\prime}}^{n,+}$ and $\phi_{i^{\prime}}^{n+1}:=\phi_{i^{\prime}}^{n+1,-}$. Since wave height $\eta_{h}$ is continuous in time, see (4.32a), we rename $\eta_{l}^{n}:=\eta_{l}^{n,+}$ and $\eta_{l}^{n+1}:=\eta_{l}^{n+1,-}$. The stability of our time integration scheme satisfies the standard criterion [64] for the Störmer-Verlet scheme. The construction and stability of such (novel) time discontinuous Galerkin finite element schemes are considered in Chapter 3 In addition, we rename $\phi_{l}^{n,+}$ as $\phi_{l}^{n}$.

By introducing variations (4.34, , 4.35) into 4.31, the final system of discrete equations for the potential and wave height becomes

$$
\left\{\begin{array}{c}
\left(M_{k l}^{n+1 / 2}+\frac{1}{2} \Delta t^{n} D_{k l}^{n+1 / 2}\right) \phi_{l}^{n+1 / 2}=M_{k l}^{n} \phi_{l}^{n} \\
-\frac{1}{2} \Delta t^{n}\left(\frac { 1 } { 2 } \left(C_{k i^{\prime} j^{\prime}}^{n+1 / 2}\left(\boldsymbol{\eta}^{n}\right) \phi_{i^{\prime}}^{n} \phi_{j^{\prime}}^{n}+C_{k l j^{\prime}}^{n+1 / 2}\left(\boldsymbol{\eta}^{n}\right) \phi_{l}^{n+1 / 2} \phi_{j^{\prime}}^{n}\right.\right. \\
\left.\left.+C_{k i^{\prime} s}^{n+1 / 2}\left(\boldsymbol{\eta}^{n}\right) \phi_{i^{\prime}}^{n} \phi_{s}^{n+1 / 2}+C_{k l s}^{n+1 / 2}\left(\boldsymbol{\eta}^{n}\right) \phi_{l}^{n+1 / 2} \phi_{s}^{n+1 / 2}\right)+g M_{k l}^{n+1 / 2} \eta_{l}^{n}\right) \\
-\frac{1}{2} \Delta t^{n}\left(E_{q}^{n+1 / 2}\left(\eta_{q}^{n}\right) \phi_{q}^{n+1 / 2} \delta_{k q}+E_{m^{\prime}}^{n+1 / 2}\left(\eta_{q}^{n}\right) \phi_{m^{\prime}}^{n} \delta_{k q}\right), \\
A_{i^{\prime} j^{\prime}}^{n+1 / 2}\left(\boldsymbol{\eta}^{n}\right) \phi_{j^{\prime}}^{n}+A_{i^{\prime} l}^{n+1 / 2}\left(\boldsymbol{\eta}^{n}\right) \phi_{l}^{n+1 / 2}=-W_{m^{\prime}}^{n+1 / 2}\left(\eta_{q}^{n}\right) \delta_{m^{\prime} i^{\prime}},
\end{array}\right.
$$

$$
\left\{\begin{array}{c}
\left(M_{k l}^{n+1 / 2}-\frac{1}{2} \Delta t^{n} D_{k l}^{n+1 / 2}\right) \eta_{k}^{n+1}=\left(M_{k l}^{n+1 / 2}+\frac{1}{2} \Delta t^{n} D_{k l}^{n+1 / 2}\right) \eta_{k}^{n} \\
+\frac{\Delta t^{n}}{2}\left(W_{q}^{n+1 / 2}\left(\eta_{q}^{n}\right)+W_{q}^{n+1 / 2}\left(\eta_{q}^{n+1}\right)\right) \delta_{q l} \\
+\frac{1}{2} \Delta t^{n}\left(A_{k l}^{n+1 / 2}\left(\boldsymbol{\eta}^{n}\right) \phi_{k}^{n+1 / 2}+A_{i^{\prime} l}^{n+1 / 2}\left(\boldsymbol{\eta}^{n}\right) \phi_{i^{\prime}}^{n}\right. \\
\left.+A_{k l}^{n+1 / 2}\left(\boldsymbol{\eta}^{n+1}\right) \phi_{k}^{n+1 / 2}+A_{i^{\prime} l}^{n+1 / 2}\left(\boldsymbol{\eta}^{n+1}\right) \phi_{i^{\prime}}^{n+1}\right), \\
A_{i^{\prime} j^{\prime}}^{n+1 / 2}\left(\boldsymbol{\eta}^{n+1}\right) \phi_{j^{\prime}}^{n+1}+A_{i^{\prime} l}^{n+1 / 2}\left(\boldsymbol{\eta}^{n+1}\right) \phi_{l}^{n+1 / 2}=-W_{m^{\prime}}^{n+1 / 2}\left(\eta_{q}^{n+1}\right) \delta_{m^{\prime} i^{\prime}},
\end{array}\right.
$$




$$
\begin{aligned}
M_{k l}^{n+1} & \phi_{l}^{n+1}=\left(M_{k l}^{n+1 / 2}-\frac{1}{2} \Delta t^{n} D_{k l}^{n+1 / 2}\right) \phi_{l}^{n+1 / 2} \\
& -\frac{1}{2} \Delta t^{n}\left(\frac { 1 } { 2 } \left(C_{k i^{\prime} j^{\prime}}^{n+1 / 2}\left(\boldsymbol{\eta}^{n+1}\right) \phi_{i^{\prime}}^{n+1} \phi_{j^{\prime}}^{n+1}+C_{k l j^{\prime}}^{n+1 / 2}\left(\boldsymbol{\eta}^{n+1}\right) \phi_{l}^{n+1 / 2} \phi_{j^{\prime}}^{n+1}\right.\right. \\
& \left.+C_{k i^{\prime} s}^{n+1 / 2}\left(\boldsymbol{\eta}^{n+1}\right) \phi_{i^{\prime}}^{n+1} \phi_{s}^{n+1 / 2}+C_{k l s}^{n+1 / 2}\left(\boldsymbol{\eta}^{n+1}\right) \phi_{l}^{n+1 / 2} \phi_{s}^{n+1 / 2}\right) \\
& \left.+g M_{k l}^{n+1 / 2} \eta_{l}^{n+1}\right) \\
& -\frac{1}{2} \Delta t^{n}\left(E_{q}^{n+1 / 2}\left(\eta_{q}^{n+1}\right) \phi_{q}^{n+1 / 2} \delta_{k q}+E_{m^{\prime}}^{n+1 / 2}\left(\eta_{q}^{n+1}\right) \phi_{m^{\prime}}^{n+1} \delta_{k q}\right),
\end{aligned}
$$

with indices $k, l, r, s \in \mathcal{N}_{F}, i^{\prime}, j^{\prime} \in \mathcal{N}_{\bar{\Omega}_{h}}, m^{\prime} \in \mathcal{N}_{W} \backslash \mathcal{N}_{F}$ and $q \in \mathcal{N}_{F} \cap \mathcal{N}_{W}$. The first and second steps, indicated by curly brackets, are implicit in time. The third step is explicit. When we consider a wave basin without wave maker, the matrices no longer depend explicitly on time. Upon elimination of the interior degrees of freedom, we have shown that the time integration method becomes the symplectic Störmer-Verlet one [64] for a nonlinear canonical Hamiltonian system. This limit motivated the approximations of the interior potential in the kinetic energy and the quadrature rules chosen for the time integrals of the Hamiltonian. Finally, we solve the implicit equations with an iterative Newton method, with tolerance $\epsilon=10^{-10}$. The resulting linear system of equations in this iteration is solved with PETSc's linear solver KSPSolve.

\subsection{Numerical Results}

\subsubsection{Verification}

\section{Nonlinear Fenton waves}

We first verify our variational finite element method against a nonlinear semi-analytical solution of the free surface water wave equations using Fenton's method [47]. In this method a Fourier approximation of the velocity potential and wave height is used at discrete points in a two-dimensional domain with a flat bottom and periodic boundary conditions in the horizontal. The resulting wave solution is a harmonic traveling wave. In this subsection, the equations of motion are solved in nondimensional form, effectively realized by taking " $g=1$ ".

We initialize the numerical simulation with a harmonic wave solution constructed by using 64 Fourier modes and simulate for 100 time periods on a regular mesh with $128 \times 16$ elements. The numerical simulation shows no decay in the wave amplitude, even after 100 time periods. The energy shows no drift, which is confirmed in Fig. 4.4 A small phase shift in the final wave profile is, however, found when compared with the initial wave profile, which is caused by the dispersion error present in the numerical scheme.

We also performed a series of tests on various meshes to verify the order of accuracy of our finite element discretization. The results are presented in Table 4.1 and show that the numerical scheme is second order accurate. 


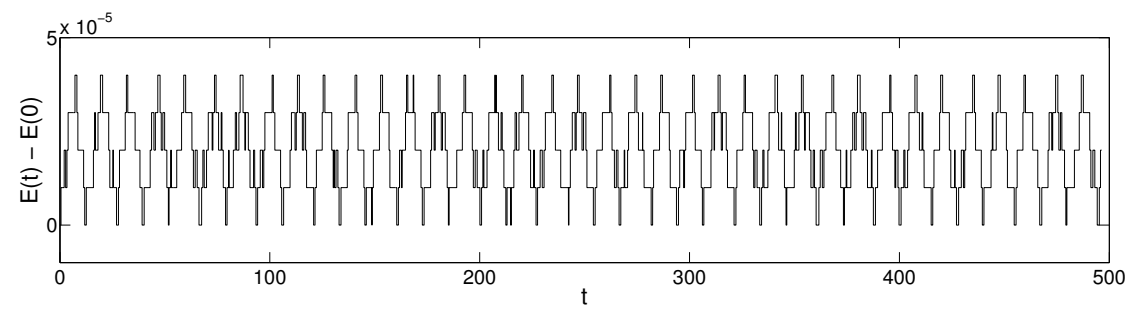

Figure 4.4: Plot of discrete energy difference $E(t)-E(0)$ versus time $t$ over 100 periods, where one period is $T_{p}=4.9636 . E(t)$ is the discrete energy at a given time $t$. It has bounded fluctuations in time and shows no drift.

\begin{tabular}{|c|c|c|c|c|c|c|c|}
\hline & & \multicolumn{2}{|l|}{$\overline{L^{2}}$} & \multicolumn{2}{|l|}{$L^{\infty}$} & \multicolumn{2}{|c|}{$\Delta E$} \\
\hline \multicolumn{2}{|l|}{ Mesh size } & $L^{2}$-error & order & $L^{\infty}$-error & order & $\Delta E$ & order \\
\hline \multirow{2}{*}{$\Delta x, \Delta z, \Delta t$} & $\eta$ & 0.052831 & - & 0.0365923 & - & 7.E-4 & - \\
\hline & $\phi$ & 0.0045161 & - & 0.0054115 & - & & \\
\hline \multirow{2}{*}{$\frac{\Delta x}{2}, \frac{\Delta z}{2}, \frac{\Delta t}{2}$} & $\eta$ & 0.0131235 & 2.009 & 0.00900984 & 2.022 & $1.7 \mathrm{E}-4$ & 2.042 \\
\hline & $\phi$ & 0.0007522 & 2.586 & 0.00118985 & 2.185 & & \\
\hline \multirow{2}{*}{$\frac{\Delta x}{4}, \frac{\Delta x}{4}, \frac{\Delta t}{4}$} & $\eta$ & 0.00327116 & 2.004 & 0.00223324 & 2.012 & $4.0 \mathrm{E}-5$ & 2.087 \\
\hline & $\phi$ & $6.2 \mathrm{E}-5$ & 3.601 & 0.00024878 & 2.258 & & \\
\hline \multirow{2}{*}{$\frac{\Delta x}{8}, \frac{\Delta x}{8}, \frac{\Delta t}{8}$} & $\eta$ & 0.00081288 & 2.009 & 0.000554157 & 2.011 & $1.0 \mathrm{E}-5$ & 2.0 \\
\hline & $\phi$ & $5.68 \mathrm{E}-6$ & 3.448 & $6.418 \mathrm{E}-5$ & 1.955 & & \\
\hline
\end{tabular}

Table 4.1: Spatial errors in the $L^{2}-$ and $L^{\infty}$-norms, order of accuracy and deviation of total energy $\Delta E$ for Fenton waves at time $10 T_{\text {per }}=49.636$. Wave height and potential are, respectively, $\eta$ and $\phi$. Time step $\Delta t=0.01551125$. The mesh is regular with $\Delta x=0.1551121$ and $\Delta z=0.25$. 


\subsubsection{Validation against laboratory data}

We have validated the numerical scheme against experimental data provided by the Maritime Research Institute Netherlands (MARIN). The experimental data are based on recordings of wave height measurements using probes at specific locations in the wave basin. There are two experimental set-ups: the first case concerns several irregular long crested waves, which are propagating over a sloping bottom, and the second test case concerns several wave groups over a flat bottom. We used the open cases classified under numbers 103001 and 202002, as opposed to other, blind cases. In both laboratory cases, a piston type wave maker is installed at the left side of the domain and a beach lies at the right side of the domain. While the piston type wave maker generates waves traveling towards the right, the laboratory beach reduces wave reflection of incoming waves via damping by wave breaking. Our present numerical scheme, instead, has a vertical solid wall on the right. This limits the validity of the numerical simulations till the time when reflected waves start to interfere with the results at the given probe locations. We investigate the capability of the numerical method to deal with (i) a sloping bottom; (ii) waves generated by the irregular movement of the wave maker; and, (iii) wave focussing.

\section{Irregular waves propagating over a sloping bottom}

First, we show a sketch of the bathymetry of the actual and numerical wave basin in Fig. 4.5 A piston-type wave maker lies at the left boundary of the domain. The wave maker always starts from rest at $x=0 \mathrm{~m}$ and can only move in the horizontal direction. To the right of the wave maker there is a flat bottom with water depth of $0.60 \mathrm{~m}$. At $x=143.41 \mathrm{~m}$ a slope of steepness $1: 20$ starts. It ends at $x=149.41 \mathrm{~m}$ at a water depth of $0.30 \mathrm{~m}$. Behind the slope there is a $24 \mathrm{~m}$ long flat bottom part. A sloping beach starts at $x=173.41 \mathrm{~m}$. As mentioned, in the numerical simulations the sloping beach is replaced with a vertical wall at $x=173.41 \mathrm{~m}$. In the numerical wave basin, the horizontal mesh movement due to the wave maker gradually diminishes, to vanish for $x>L_{w}=40 \mathrm{~m}$.

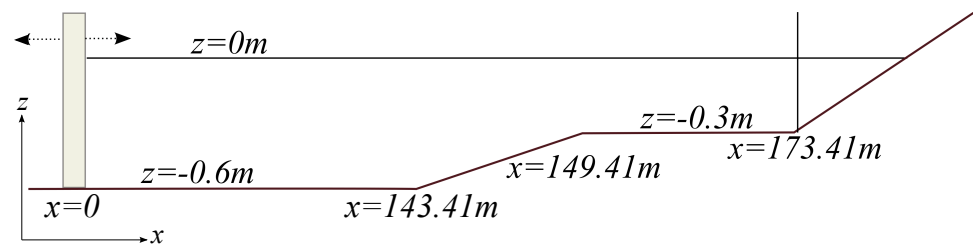

Figure 4.5: Sketch of the bathymetry for Case 103001 with a beach in the laboratory basin and a wall at the start of the beach in the numerical basin.

The measured piston motion used in the numerical simulations is shown in Fig. 4.6 Wave elevations are measured at various probes, with their positions given in Table 4.2 


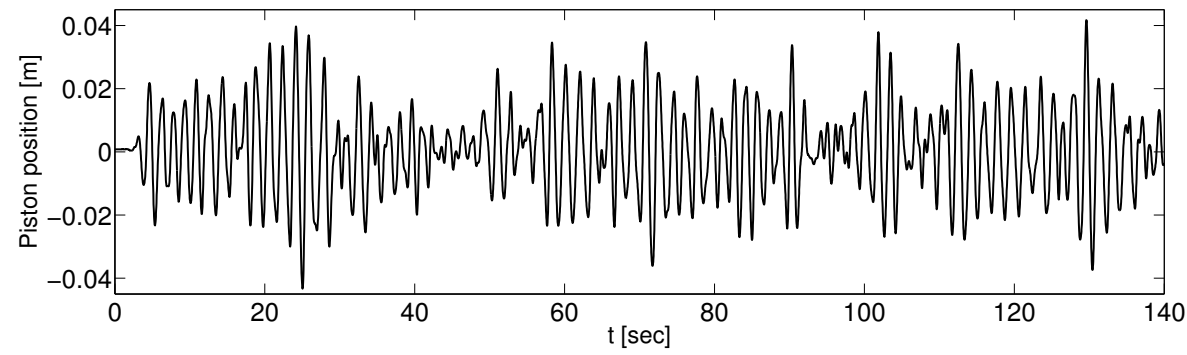

Figure 4.6: Measured wave maker motion for Case 103001.

\begin{tabular}{l|l|l|l|l|l|l|l|l}
\hline Wave probe & 1 & 2 & 9 & 12 & 13 & 15 & 17 & 25 \\
\hline Distance $(\mathrm{m})$ & 39.15 & 78.8 & 102.12 & 143.41 & 146.43 & 149.41 & 157.74 & 172.89 \\
\hline
\end{tabular}

Table 4.2: Locations of probes for wave height measurements in Case 103001.

For Case 103001, the results of the computations for the variable bottom are summarized as follows.

For this test case, the motion of the wave maker, shown in Fig. 4.6, generates highly irregular waves. A fixed time step, $\Delta t=0.00125 \mathrm{~s}$, is used in the numerical simulations with $N_{x}=12000$ equidistantly distributed points in the horizontal direction. In the vertical direction we have $N_{z}=10$ points distributed exponentially, such that the smallest element lies at the free surface.

A comparison of the experiments and numerical simulations at all available probes is shown in Fig. 4.7 The experimental ("red") and numerical results ("blue") generally agree well. The only larger mismatch observed is found in Fig.4.7(c,h), presenting results for Probes 9 and 25. The mismatch for Probe 9 is due to a known error in the documented position of this probe, while Probe 25 simply lies too close to the beach or vertical wall.

Since the incoming waves reach the solid wall around $t=110 \mathrm{~s}$, comparisons with experimental data have been made only till $t=120$ s, to prevent contamination from the simulated wave reflections off the solid wall. As can be seen for Probe 25 at $x=$ $172.89 \mathrm{~m}$, located close to the reflecting boundary, the comparison stops to be accurate after $t=110 \mathrm{~s}$. To obtain a rough estimate of the time when the reflected waves will reach this probe, we use the frequency relation for linear water waves with $\omega^{2}=g k \tanh \left(k H_{0}\right)$ and $H_{0}=0.6 \mathrm{~m}$. As we can see from Figure 4.8 (a), there are a large number of long waves with frequencies around $\omega=3 / \mathrm{s}$. The relevant wavenumber is $k=1.362 / \mathrm{m}$. For an average depth $H_{0}=0.6 \mathrm{~m}$, we obtain a phase velocity $c_{p} \equiv \omega / k=2.42 \mathrm{~m} / \mathrm{s}$, just short of the fastest, long wave speed $c_{p}=\sqrt{g H_{0}} \approx 2.45 \mathrm{~m} / \mathrm{s}$. The distance of $173.41 \mathrm{~m}$ is then covered in about 70s at this frequency, which is an adequate time estimate to halt the numerical simulations before they are contaminated by reflections from the end wall.

To validate our numerical results further, we compute spectra using a fast Fourier 


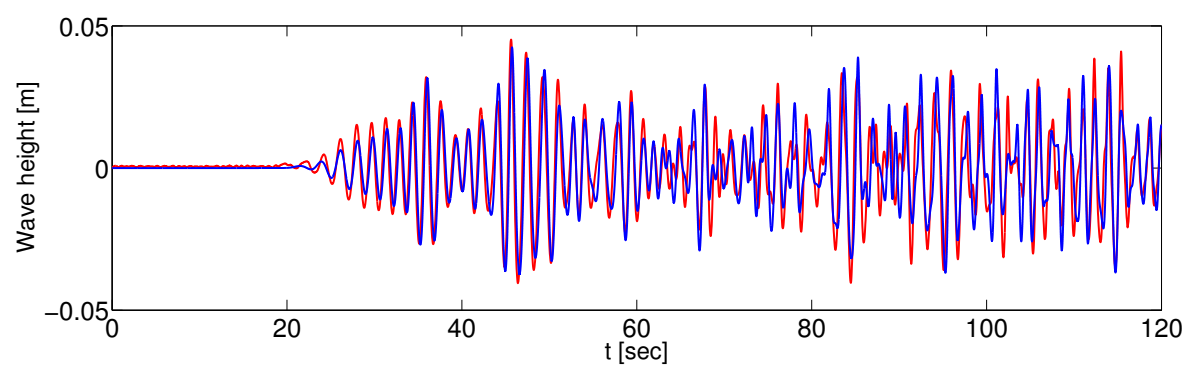

(a) $x=39.15 \mathrm{~m}$.

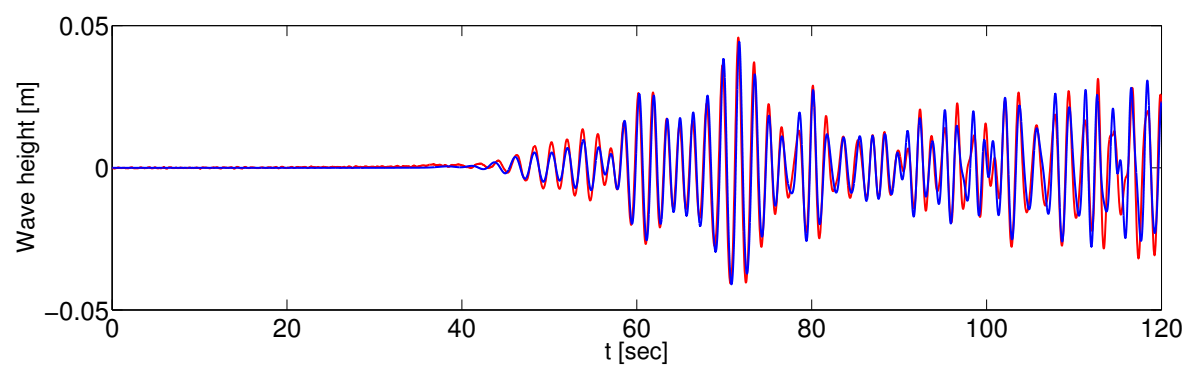

(b) $x=78.80 \mathrm{~m}$.

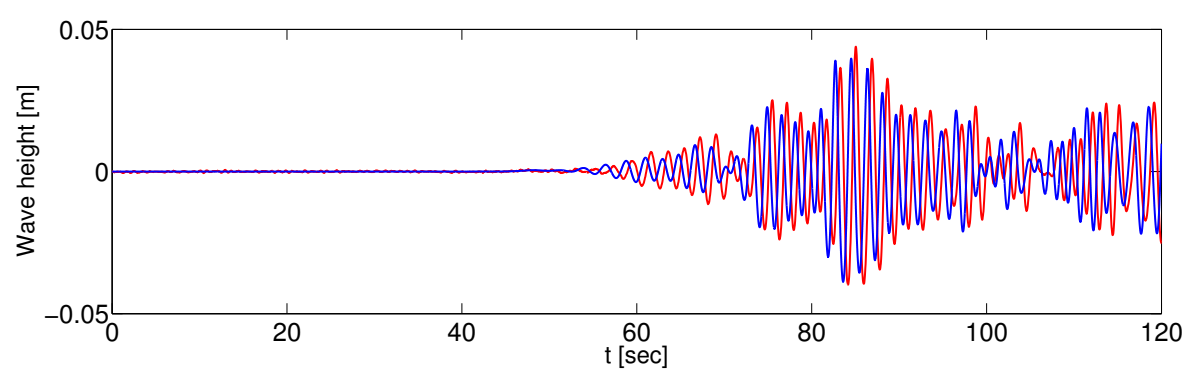

(c) $x=102.12 \mathrm{~m}$.

Figure 4.7: Comparison of wave heights in the experiment and numerical simulation at all available probe locations for test case 103001 with a sloping bottom. The numerical simulation is performed on a mesh of $12000 \times 10$ elements with time step $\Delta t=0.00125 \mathrm{~s}$. Experiments are in "red" and numerical simulations in "blue". 


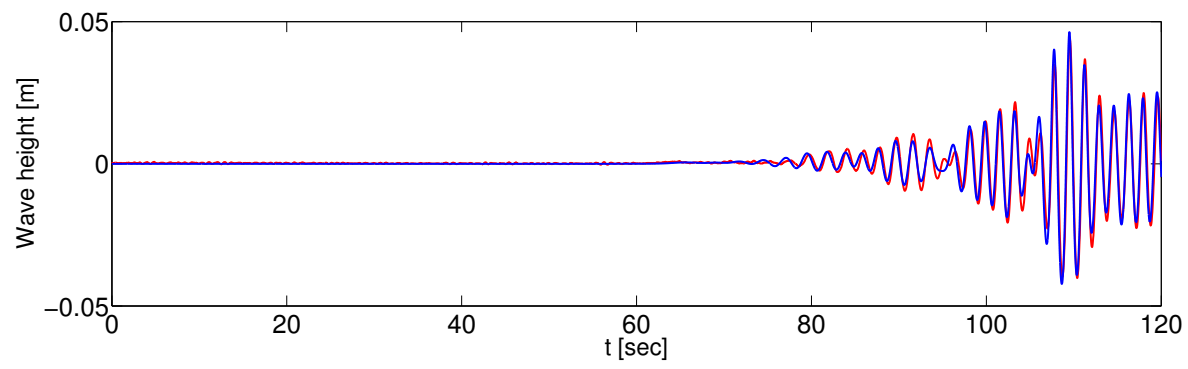

(d) $x=143.41 \mathrm{~m}$.

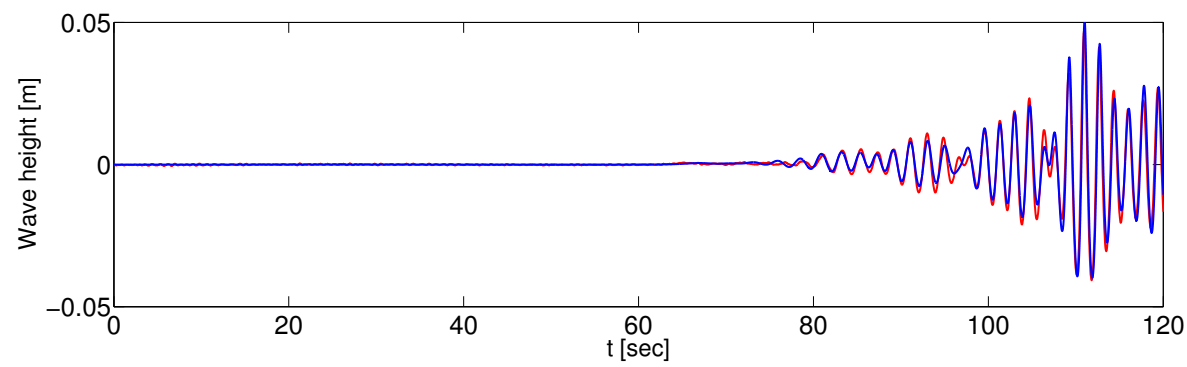

(e) $x=146.43 \mathrm{~m}$.

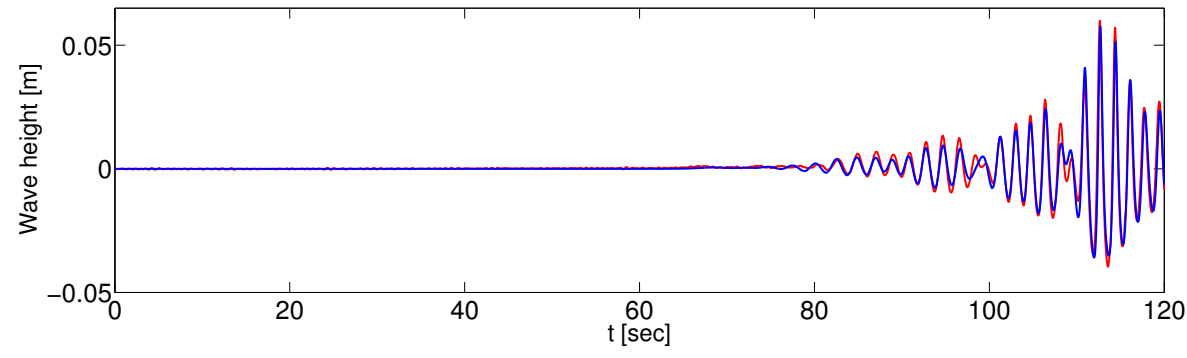

(f) $x=149.41 \mathrm{~m}$.

Figure 4.7 continued: Continued: comparison of wave heights in the experiment and numerical simulation for test case 103001. Experiments are in "red" and numerical simulations in "blue". 


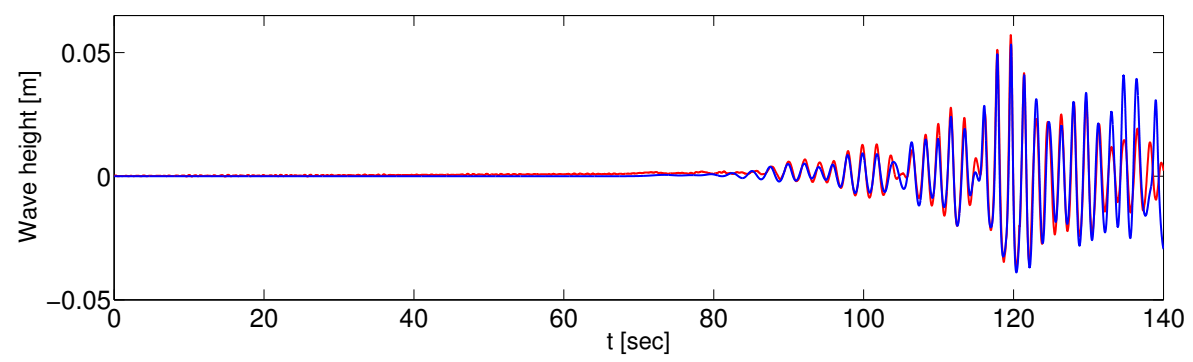

(g) $x=157.74 \mathrm{~m}$.

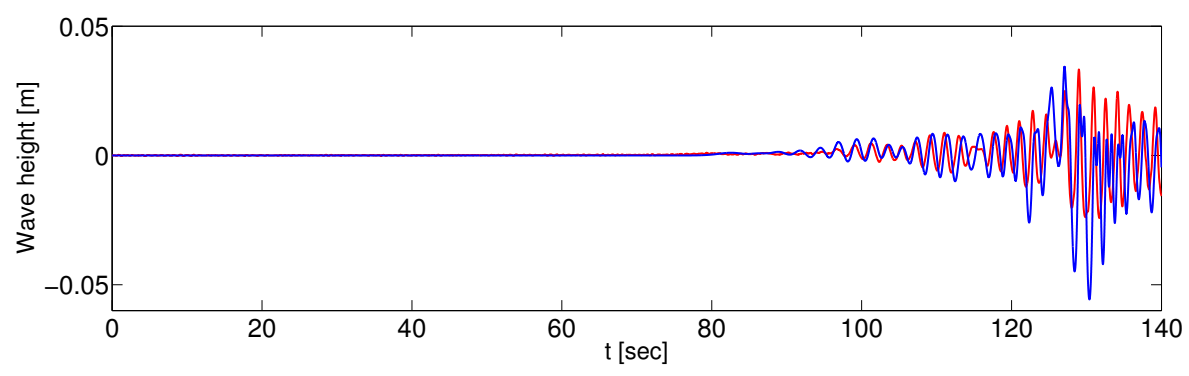

(h) $x=172.89 \mathrm{~m}$.

Figure 4.7 continued: Continued: comparison of wave heights in the experiment and numerical simulation for the test case 103001. Experiments are in "red" and numerical simulations in "blue". 
transform (FFT) to analyze the data from the numerical simulations and the laboratory experiments. We consider three probes: Probe 1 at $x=39.15 \mathrm{~m}$ located at the beginning of the basin, Probe 2 at $x=78.80 \mathrm{~m}$, and Probe 9 at $x=102.12 \mathrm{~m}$ in the middle. We can observe in Fig. 4.8 that the comparison is good for all three probes (despite the known error in the recorded position of Probe 9).

The spectral analysis is also useful to estimate the mesh size and time step before the numerical simulation is performed. Considering the spectra of the laboratory data at the first probe ("red") in Fig. 4.8.a), we see that frequencies up to $\omega=11 / \mathrm{s}$ are relevant and that the rest can be neglected. After an analysis of wave heights at all the probes, we stay on the safe side by taking a limiting frequency $\omega=15 / \mathrm{s}$. The time period of the shortest wave is then $T=2 \pi / \omega=0.419$ s. Taking at least 10 time steps per period, we obtain a time step estimate $\Delta t=0.0419 \mathrm{~s}$. Using the linear frequency relation for the intermediate depth $H_{0}$, we find the corresponding wavenumber $k=22.94 / m=2 \pi / \lambda$. Taking at least 10 points per shortest wave length $\lambda=0.274 \mathrm{~m}$, we obtain that the required horizontal mesh size is $\Delta x=0.0274 \mathrm{~m}$, which gives a number of elements equal to $N_{x}=173.41 / \Delta x \approx 6500$. Snapshots of water waves at various moments of time are presented in Fig. 4.9 .

\section{Wave groups propagating over a flat bottom}

In test Case 202002, the wave basin has a flat bottom and the horizontally-driven wave maker starts from rest at $x=0 \mathrm{~m}$. The wave basin is $1 \mathrm{~m}$ deep and $195.4 \mathrm{~m}$ long. The measured piston motion is shown in Fig. 4.10. Wave elevations are measured at various probes, see Table. 4.3. Measured and computed wave heights at the probes are seen to be in good agreement, from Fig. 4.11. The piston motion generates a slow moving wave group followed by a fast moving wave group, resulting in wave focussing around $t=109.56 \mathrm{~s}$ and $x=50 \mathrm{~m}$, see Fig. 4.12

\begin{tabular}{l|l|l|l|l|l|l}
\hline Wave probe number & 1 & 2 & 3 & 4 & 5 & 6 \\
\hline Distance $(\mathrm{m})$ & 10 & 20 & 40 & 49.5 & 50 & 54 \\
\hline
\end{tabular}

Table 4.3: Location of probes for wave height measurements in Case 202002.

Spectral analysis is again used to estimate the required mesh size and time step. We analyzed the laboratory data for all available probes. Considering the spectrum of the second probe ("red") placed at $x=20 \mathrm{~m}$, see Figure 4.13 a), we note that frequencies up to $\omega=11 / \mathrm{s}$ are relevant. This limiting frequency corresponds to a time pe$\operatorname{riod} T=2 \pi / \omega=0.571 \mathrm{~s}$. With 10 time steps per period, this leads to a time step of $\Delta t=0.0571 \mathrm{~s}$. Given the dispersion relation $\omega=\sqrt{g k \tanh \left(k H_{0}\right)}$ with depth $H_{0}=1 \mathrm{~m}$, the corresponding wavenumber $k=2 \pi / \lambda=12.34 / \mathrm{m}$. Using 10 points for this wave length $\lambda=0.509 \mathrm{~m}$, we estimate a horizontal mesh size of $\Delta x=0.0509 \mathrm{~m}$. We know, however, from the laboratory experiment that a splash occurs around this probe, so a 


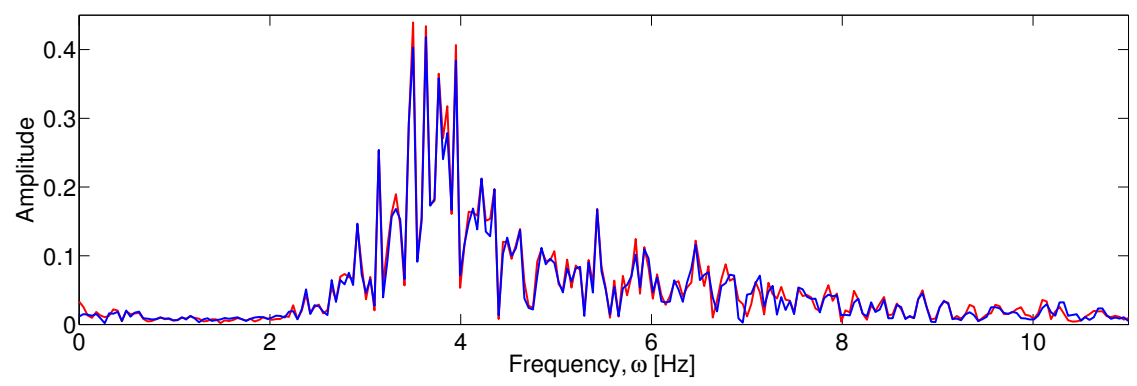

(a) $x=39.15 \mathrm{~m}$.

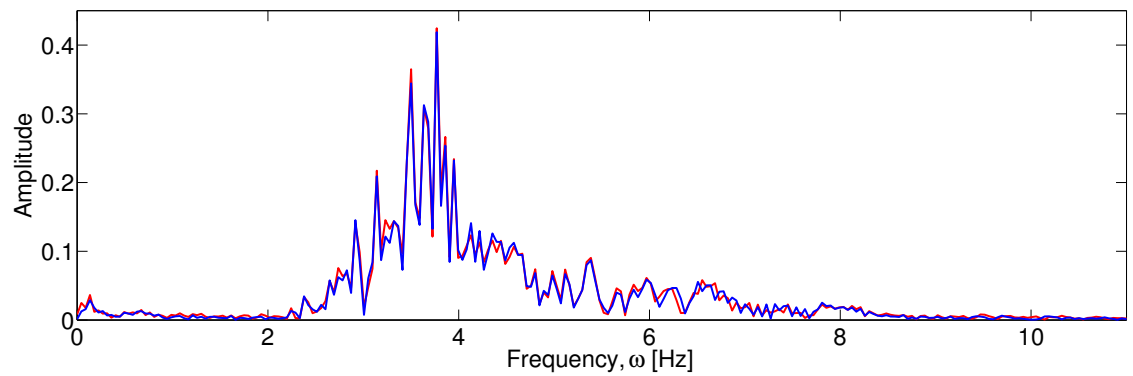

(b) $x=78.80 \mathrm{~m}$.

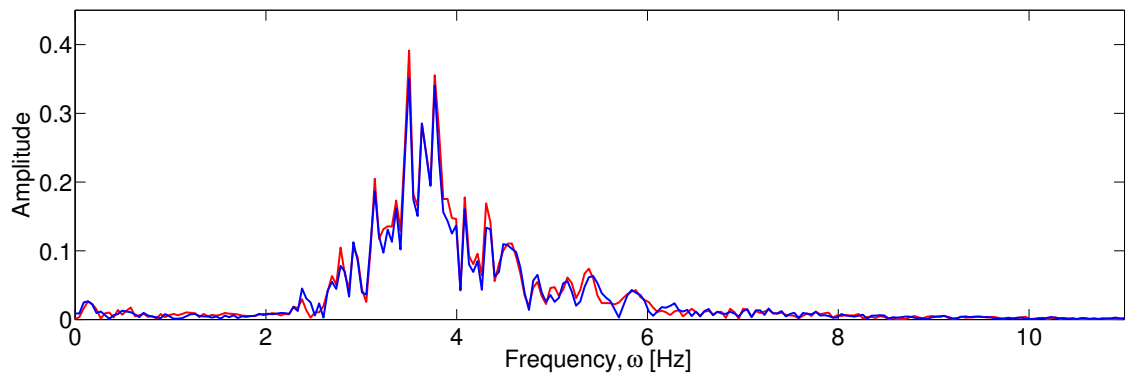

(c) $x=102.12 \mathrm{~m}$.

Figure 4.8: Time spectra of measured wave heights ("red") and computed wave heights ("blue") at Probes 1, 2 and 9. 


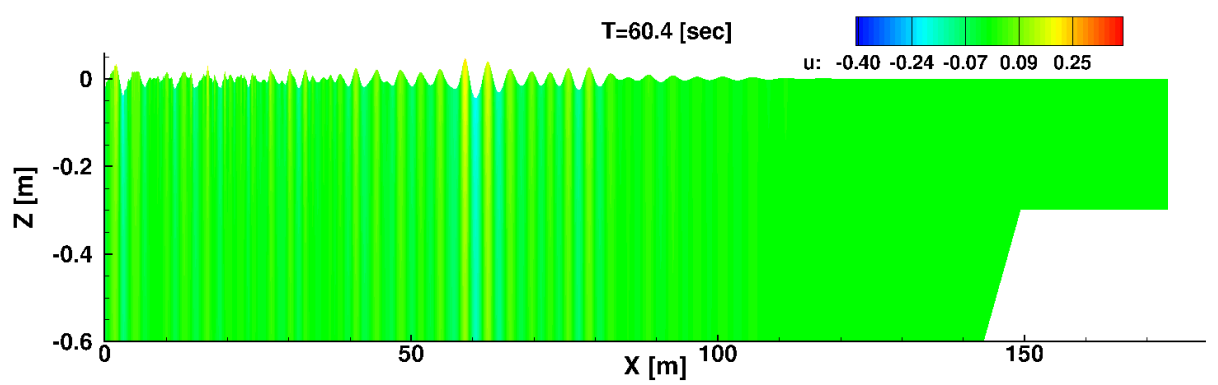

(a)

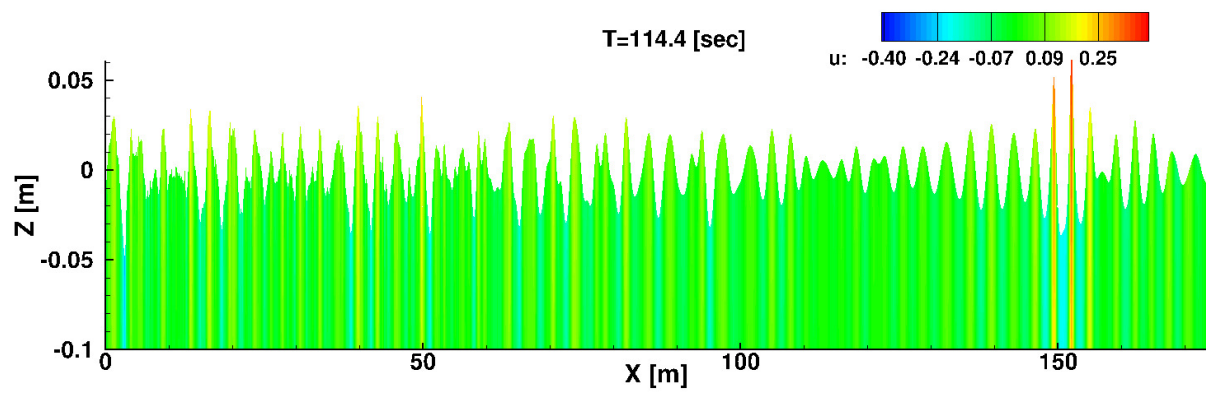

(b)

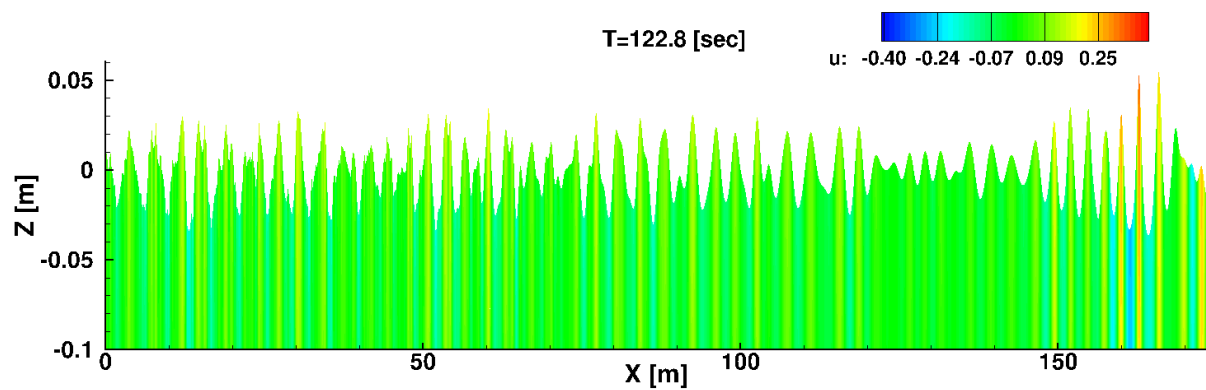

(c)

Figure 4.9: Snapshots of the simulated domain and horizontal velocity are shown at times $t=60.4 \mathrm{~s}, t=114.4 \mathrm{~s}$ and $t=122.8 \mathrm{~s}$ for the variable bottom test Case 103001 . We observe highly irregular waves generated by the wave maker and reflections due to a solid wall at the right end of the domain. A mesh of $12000 \times 10$ elements with time step $\Delta t=0.00125 \mathrm{~s}$ is used. (a) Complete domain and (b,c) zoom-ins at the free surface. 


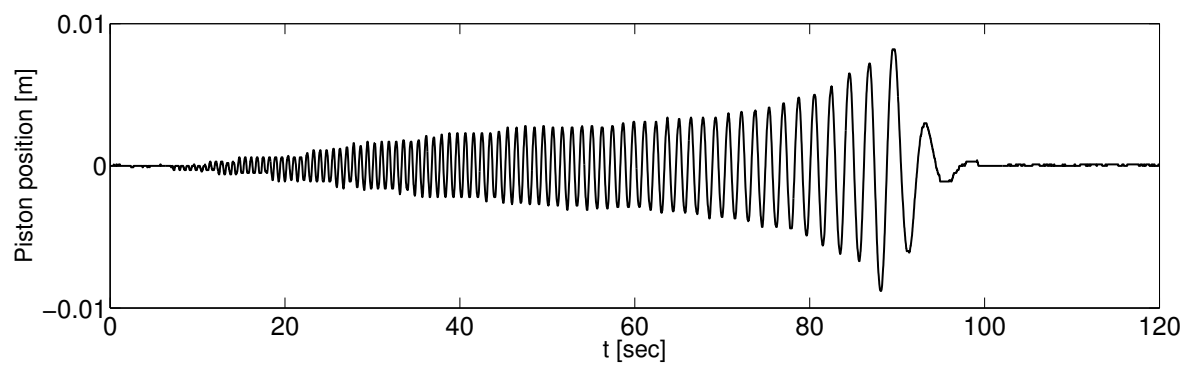

Figure 4.10: Wave maker motion for Case 202002.

more restrictive time step follows from the "red" laboratory spectrum of the fifth probe at $x=50 \mathrm{~m}$. In Figure 4.13 (c), a peak is observed at $\omega=36 / \mathrm{s}$. For this limiting frequency $\omega=36 / \mathrm{s}$ the time period of the shortest wave is $T=0.1745 \mathrm{~s}$ and the wave length is $\lambda=0.0385 \mathrm{~m}$. The associated time step is $\Delta t=0.01745 \mathrm{~s}$ and mesh size $\Delta x=0.00385 \mathrm{~m}$. The mesh size should thus be much smaller near the fifth probe than near the first probe. In order to estimate the vertical structure, we perform an analysis of linear waves for the peak frequency $\omega=36 / \mathrm{s}$. An exponential distribution of $N_{z}=20$ points proves to be sufficient, when we resolve the vertical structure of the corresponding linear free mode for $\omega=36 / \mathrm{s}$.

To save computing time, we have adapted the mesh to satisfy the mesh size requirements around each probe. At the beginning of the domain $\Delta x=0.0509 \mathrm{~m}$ is sufficient everywhere, except around $x=50.5 \mathrm{~m}$ where we require at least $\Delta x=0.00385 \mathrm{~m}$. The parameters for the calculations are selected as: $\Delta x=0.01559 \mathrm{~m}$ in the first part of the domain and $\Delta x=0.0027 \mathrm{~m}$ in the splash zone. Initially, the vertical space step ranges from $\Delta z=0.098222$ near the flat bottom to $\Delta z=0.00247959$ near the free surface. The time step is taken constant $\Delta t=0.001 \mathrm{~s}$. As we can see for the second probe in Fig. 4.13 a), the comparison between the experimental spectra ("red") and those of the numerical simulation ("blue") is good. Unfortunately, the numerical simulations do not capture the peak at $\omega=36 / \mathrm{s}$ seen in the experimental spectrum for the fifth probe, see Fig. 4.13 b). The origin of this peak in the experimental spectrum remains unclear. In the only other numerical study available on Case 202002 [91], this high frequency part of this spectrum is absent.

\subsection{Application of new third order symplectic time inte- grator}

In this section we apply the novel third order integrator derived in Chapter 3 to the numerical method developed in the current chapter for nonlinear water waves. In contrast to the rest of this chapter, we only consider an autonomous variational principle, i.e. a 


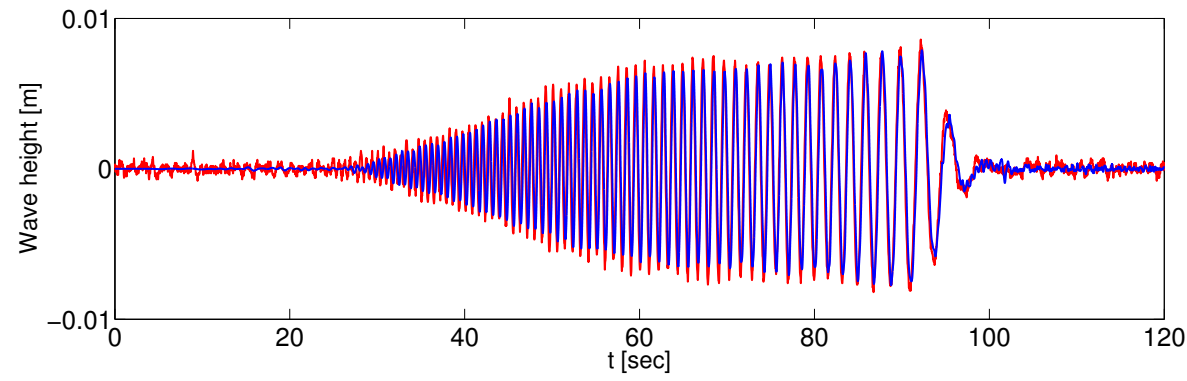

(a) $x=10 \mathrm{~m}$

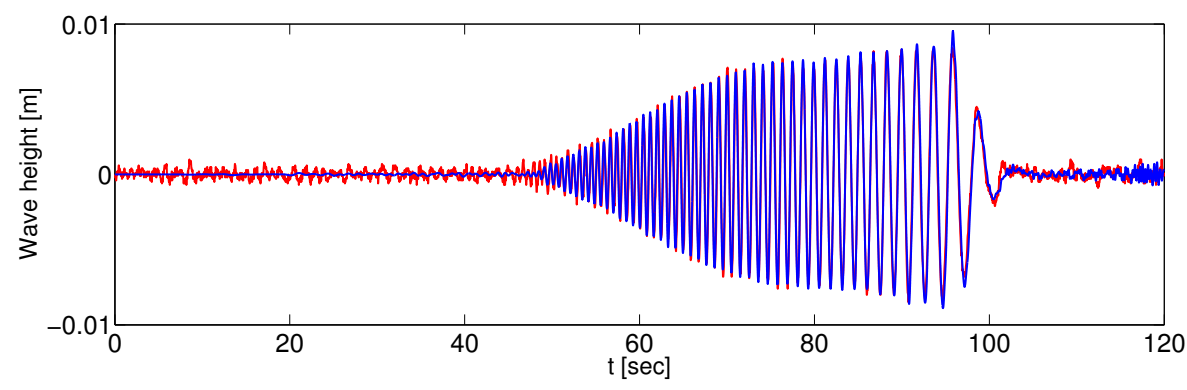

(b) $x=20 \mathrm{~m}$.

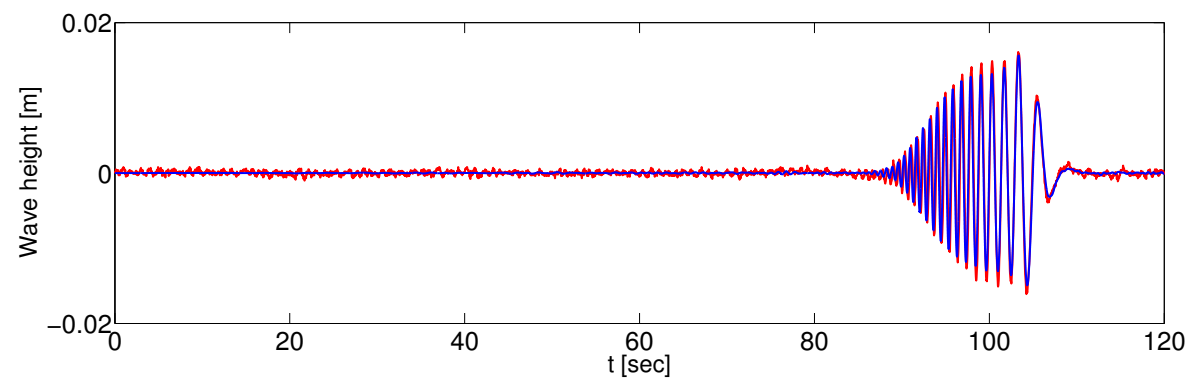

(c) $x=40 \mathrm{~m}$.

Figure 4.11: Comparison of wave heights in experiments and numerical simulations at all available probe locations for test Case 202002 with a flat bottom. The numerical simulation is performed on a nonuniform mesh with $6000 \times 20$ elements and time step $\Delta t=0.001 \mathrm{~s}$. Experimental data are shown in "red" and numerical ones in "blue". 


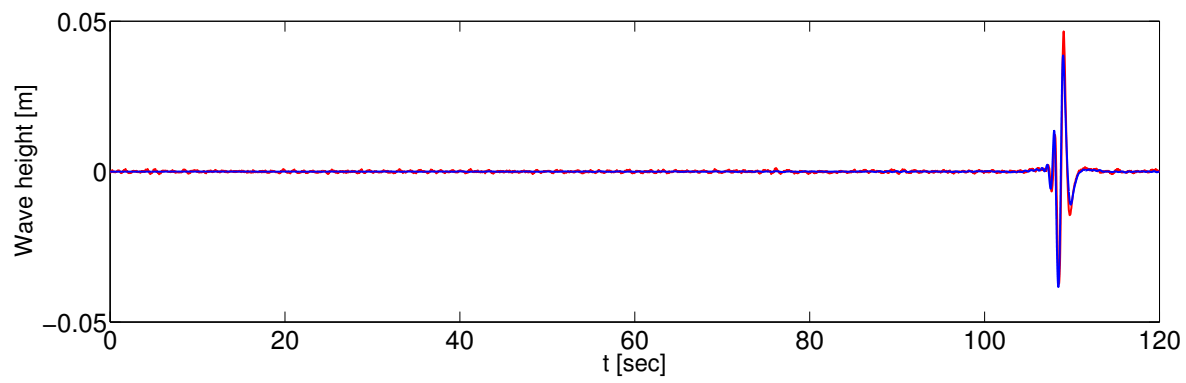

(d) $x=49.5 \mathrm{~m}$.

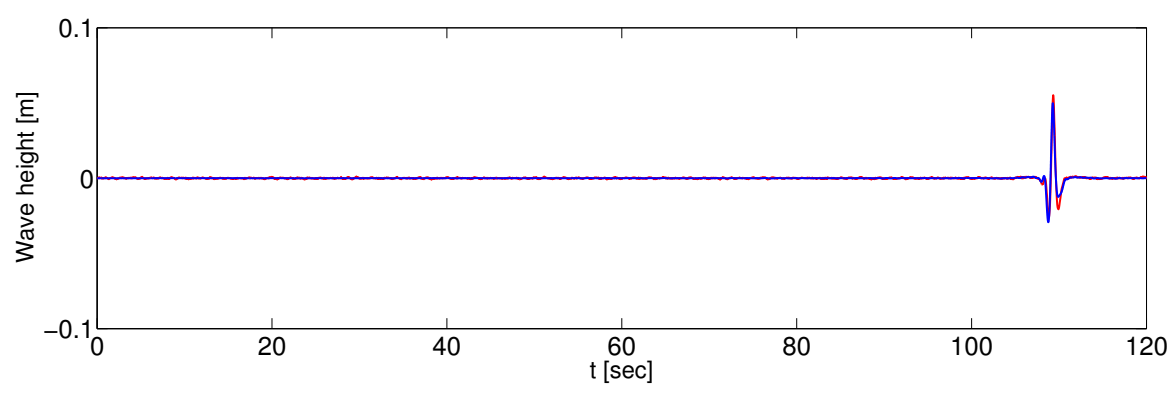

(e) $x=50 \mathrm{~m}$.

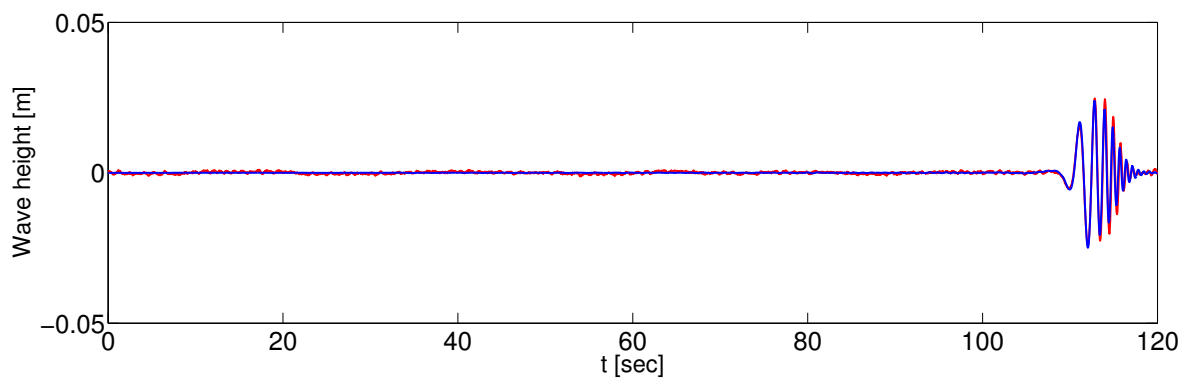

(f) $x=54 \mathrm{~m}$

Figure 4.11 continued: Continued: comparison of wave heights in experiments and numerical simulations for test Case 202002. Experimental data in "red" and numerical ones in "blue". 


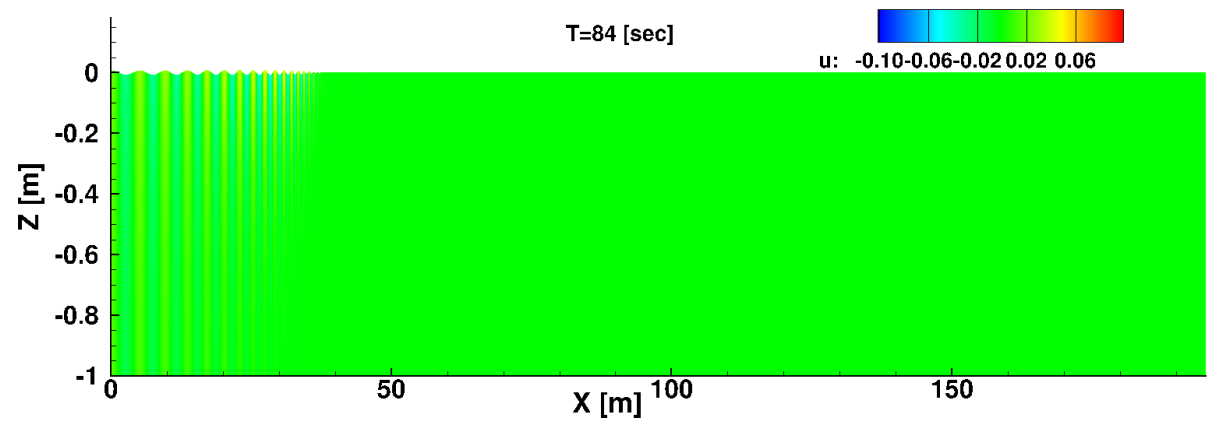

(a)

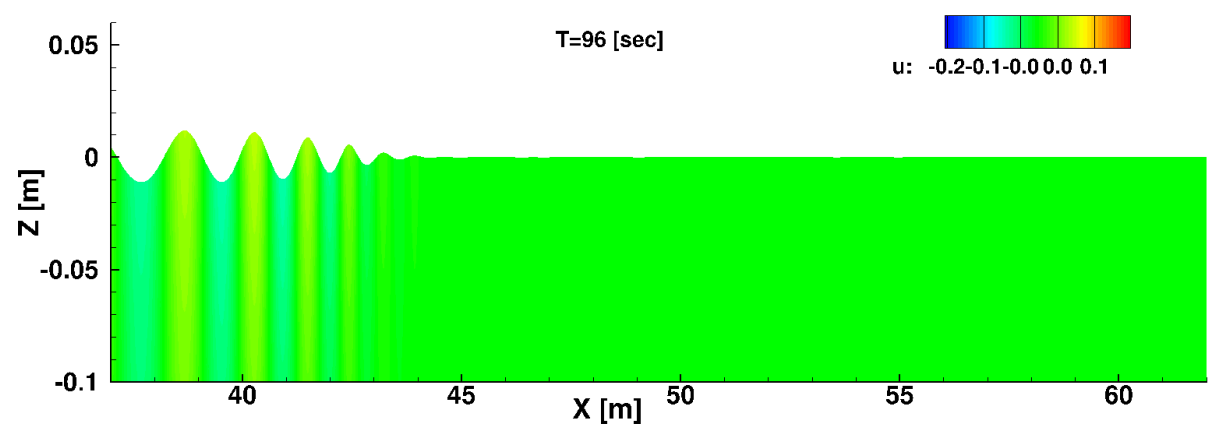

(b)

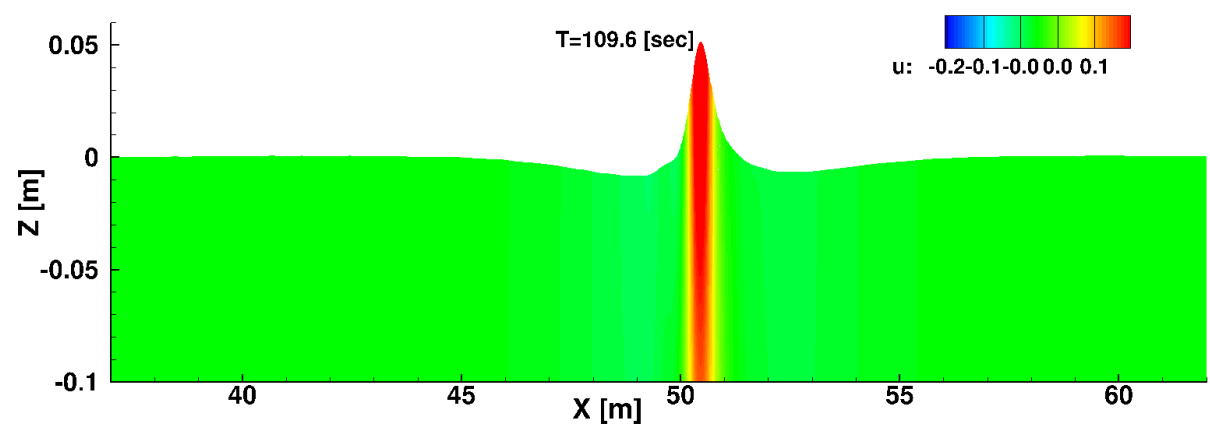

(c)

Figure 4.12: Snapshots of domain and horizontal velocity at different times for the flat bottom Case 202002: a fast moving wave group is overtaking a slow moving wave group, resulting in wave focussing at $t=109.56 \mathrm{~s}$ around $x=50.75 \mathrm{~m}$. (a) Complete domain and (b)-(d) zoom-ins at the free surface. 


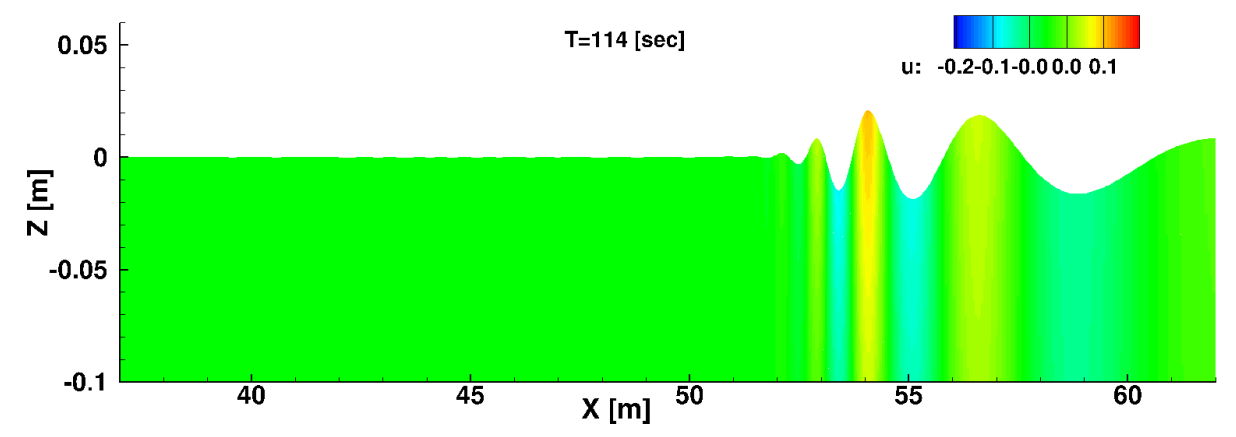

(d)

Figure 4.12 continued: Continued.

wave maker motion is not included.

\subsubsection{Nonlinear potential flow water waves}

We investigate the performance of the third order symplectic time integrator 3.38 to compute nonlinear potential flow water waves in a water basin. The dynamics of the water waves can be embedded in Miles' variational principle [106]. For a wave basin with solid boundaries Miles' variational principle 4.1) takes the form

$$
\begin{aligned}
0 & =\delta \mathcal{L}\left(\phi, \eta, \phi_{s}\right) \\
& :=\delta \int_{0}^{T}\left(\int_{0}^{L}\left(\phi_{s} \frac{\partial \eta}{\partial t}-\frac{1}{2} g\left(\eta^{2}-b^{2}\right)\right) \mathrm{d} x-\int_{0}^{L} \int_{-b(x)}^{\eta(x, t)} \frac{1}{2}|\nabla \phi|^{2} \mathrm{~d} z \mathrm{~d} x\right) \mathrm{d} t .
\end{aligned}
$$

The domain $\Omega=\{0<x<L,-b(x)<z<\eta(x, t)\} \subset \mathbb{R}^{2}$ is time dependent due to the movement of the free surface boundary $S_{F}: z=\eta(x, t)$. The bottom is fixed $S_{B}: z=$ $-b(x)$, and also the solid walls to the left and right of the basin: $S_{L}: x=0, x=L$ are fixed. The total energy of the system - the Hamiltonian - takes the form

$$
H\left(\phi, \eta, \phi_{s}\right):=\int_{0}^{L}\left(\frac{1}{2} g\left(\eta^{2}-b^{2}\right)\right) \mathrm{d} x+\int_{0}^{L} \int_{-b(x)}^{\eta(x, t)} \frac{1}{2}|\nabla \phi|^{2} \mathrm{~d} z \mathrm{~d} x .
$$

By taking the variational derivatives with respect to $\phi, \eta, \phi_{s}$ and the velocity potential at the fixed boundaries $\phi(0, z, t), \phi(L, z, t), \phi(x,-b, t)$, in 4.37) we obtain the potential flow equation with nonlinear boundary conditions. The equations are analogous to the ones described by 4.6 under the condition $R(t) \equiv 0$. 


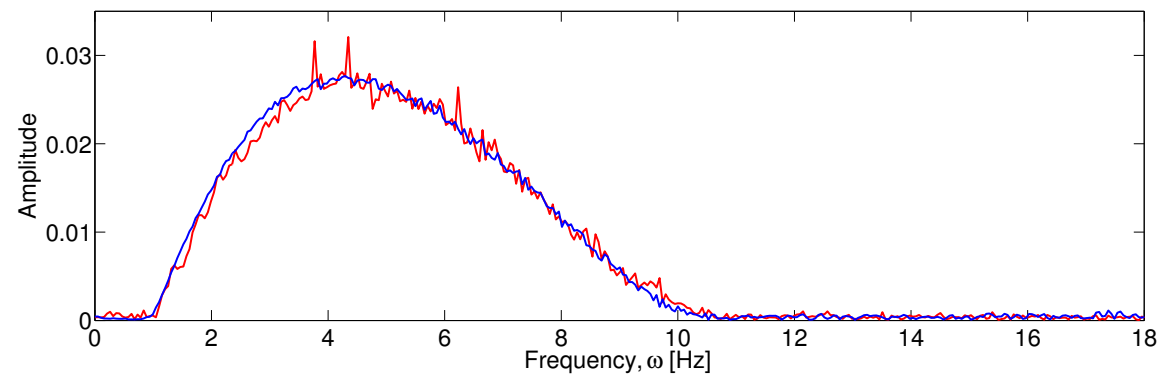

(a) $x=20 \mathrm{~m}$.

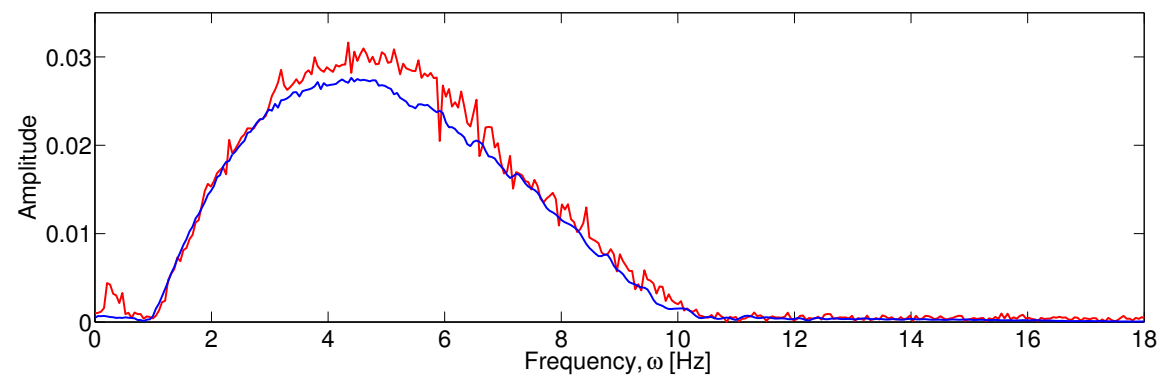

(b) $x=40 \mathrm{~m}$.

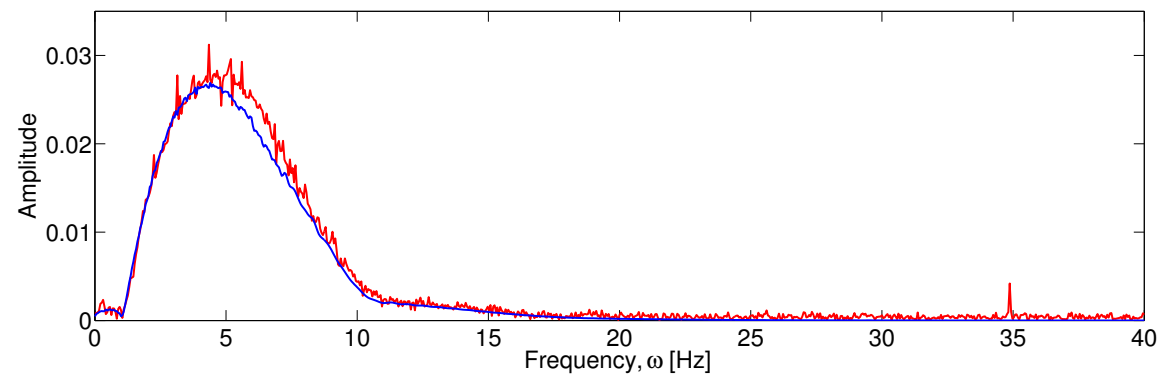

(c) $x=50 \mathrm{~m}$.

Figure 4.13: Time spectra of measured wave ("red") and computed wave heights ("blue") for Case 202002 at Probes 2, 3 and 5. 
In the autonomous case space discrete variational principle (4.13) takes the form

$$
\begin{aligned}
0 & =\delta \int_{0}^{T} L\left(\phi_{i^{\prime}}, \phi_{k}, \eta_{l}\right) \mathrm{d} t \\
& =\delta \int_{0}^{T}\left(M_{k l} \phi_{k} \frac{\mathrm{d} \eta_{l}}{\mathrm{~d} t}-\frac{1}{2} g M_{k l} \eta_{k} \eta_{l}-\frac{1}{2} A_{i j} \phi_{i} \phi_{j}\right) \mathrm{d} t,
\end{aligned}
$$

with the finite element matrices defined as in 4.14) with $R(t) \equiv 0$. The basis functions in the interior remain time dependent as they follow the free surface movement, while the basis functions at the free surface become time independent, therefore the matrix $D_{k l}$ is not present. The Hamiltonian 4.38 is approximated as

$$
H(\boldsymbol{\phi}, \boldsymbol{\eta})=\frac{1}{2} g M_{k l} \eta_{k} \eta_{l}+\frac{1}{2} A_{i j} \phi_{i} \phi_{j}
$$

In the spatial discretization, indices $i, j=1, \ldots, N_{n}$, denote nodes anywhere in the mesh, indices $k, l=1, \ldots, N_{f}$ denote the free surface nodes, and nodes indexed by $i^{\prime}, j^{\prime}$ denote interior nodes (all nodes excluding the free surface ones).

Further, as it is done in (4.21), we need to approximate the variables in time. We choose within each time interval $t \in\left(t^{n}, t^{n+1}\right)$, with $t^{n+1}=t^{n}+\Delta t$, a quadratic expansion in time analogous to the expansion (3.32). Then the free surface $\eta$ and potential $\phi$ are approximated as:

$$
\begin{aligned}
\eta_{l} & =\frac{\left(t^{n}+t^{n+1}-2 t\right)\left(t^{n+1}-t\right)}{\Delta t^{2}} \eta_{l}^{n,+}+\frac{4\left(t-t^{n}\right)\left(t^{n+1}-t\right)}{\Delta t^{2}} \eta_{l}{ }^{n+1 / 2} \\
& -\frac{\left(t^{n}+t^{n+1}-2 t\right)\left(t-t^{n}\right)}{\Delta t^{2}} \eta_{l}^{n+1,-} \\
\phi_{i} & =\frac{\left(t^{n}+t^{n+1}-2 t\right)\left(t^{n+1}-t\right)}{\Delta t^{2}} \phi_{i}{ }^{n++}+\frac{4\left(t-t^{n}\right)\left(t^{n+1}-t\right)}{\Delta t^{2}} \phi_{i}{ }^{n+1 / 2} \\
& -\frac{\left(t^{n}+t^{n+1}-2 t\right)\left(t-t^{n}\right)}{\Delta t^{2}} \phi_{i}{ }^{n+1,-} .
\end{aligned}
$$

Using approximations 4.41a we obtain an approximation of the continuous variational principle (4.37) analogously as done for the discrete variational principle (3.34), 
resulting in the discrete minimization problem

$$
\begin{aligned}
& 0=\delta \sum_{n=0}^{N-1} L\left(\phi_{i}{ }^{n,+}, \eta_{l}{ }^{n,+}, \phi_{i}{ }^{n+1 / 2}, \eta_{l}{ }^{n+1 / 2}, \phi_{i}{ }^{n+1,-}, \eta_{l}{ }^{n+1,-}\right) \\
& =\delta \sum_{n=0}^{N-1} M_{k l}\left(\frac{1}{6} \phi_{k}{ }^{n++}\left(-3 \eta_{l}{ }^{n++}+4 \eta_{l}{ }^{n+1 / 2}-\eta_{l}{ }^{n+1,-}\right)\right. \\
& \left.+\frac{2}{3} \phi_{k}{ }^{n+1 / 2}\left(-\eta_{l}{ }^{n,+}+\eta_{l}{ }^{n+1,-}\right)+\frac{1}{6} \phi_{k}{ }^{n+1,-}\left(\eta_{l}{ }^{n,+}-4 \eta_{l}{ }^{n+1 / 2}+3 \eta_{l}{ }^{n+1,-}\right)\right) \\
& -\frac{\Delta t}{6}\left(H\left(\phi_{i}{ }^{n,+}, \eta_{l}{ }^{n,+}\right)+4 H\left(\phi_{i}{ }^{n+1 / 2}, \eta_{l}{ }^{n+1 / 2}\right)+H\left(\phi_{i}{ }^{n+1,-}, \eta_{l}{ }^{n+1,-}\right)\right) \\
& -\delta \sum_{n=-1}^{N-1} \frac{1}{2} M_{k l}\left(\eta_{l}{ }^{n+1,-}-\eta_{l}{ }^{n+1,+}\right)\left(\phi_{k}{ }^{n+1,-}+\phi_{k}{ }^{n+1,+}\right) .
\end{aligned}
$$

Variation of 4.42 with respect to the independent variables $\phi_{k}^{n,+}, \eta_{l}^{n,+}, \phi_{k}^{n+1 / 2}, \phi_{j^{\prime}}^{n+1 / 2}$, $\eta_{l}^{n+1 / 2}, \phi_{k}^{n+1,-}, \phi_{j^{\prime}}^{n+1,-}, \eta_{l}^{n+1,-}$ gives the following variational discretization

$$
\begin{aligned}
& \int M_{k l} \eta_{l}^{n+1 / 2}=\frac{3}{4} M_{k l} \eta_{l}^{n,-}+\frac{1}{4} M_{k l} \eta_{l}^{n,+} \\
& +\frac{\Delta t}{4}\left(\frac{\partial H\left(\phi_{i}{ }^{n,+}, \eta_{l}{ }^{n,+}\right)}{\partial \phi_{k}^{n,+}}+\frac{\partial H\left(\phi_{i}{ }^{n+1 / 2}, \eta_{l}{ }^{n+1 / 2}\right)}{\partial \phi_{k}^{n+1 / 2}}\right), \\
& \left\{M_{k l} \phi_{k}^{n+1 / 2}=\frac{3}{4} M_{k l} \phi_{k}^{n,-}+\frac{1}{4} M_{k l} \phi_{k}^{n,+}\right. \\
& -\frac{\Delta t}{4}\left(\frac{\partial H\left(\phi_{i}{ }^{n,+}, \eta_{l}{ }^{n,+}\right)}{\partial \eta_{l}^{n,+}}+\frac{\partial H\left(\phi_{i}{ }^{n+1 / 2}, \eta_{l}{ }^{n+1 / 2}\right)}{\partial \eta_{l}^{n+1 / 2}}\right), \\
& A_{i^{\prime} j^{\prime}}\left(\boldsymbol{\eta}^{n+1 / 2}\right) \phi_{i^{\prime}}^{n+1 / 2}=-A_{k j^{\prime}}\left(\boldsymbol{\eta}^{n+1 / 2}\right) \phi_{k}^{n+1 / 2}, \\
& M_{k l} \eta_{l}^{n+1,-}=M_{k l} \eta_{l}^{n,+}+\Delta t \frac{\partial H\left(\phi_{i}^{n+1 / 2}, \eta_{l}^{n+1 / 2}\right)}{\partial \phi_{k}^{n+1 / 2}}, \\
& M_{k l} \phi_{k}^{n+1,-}=M_{k l} \phi_{k}^{n,+}-\Delta t \frac{\partial H\left(\phi_{i}{ }^{n+1 / 2}, \eta_{l}{ }^{n+1 / 2}\right)}{\partial \eta_{l}^{n+1 / 2}}, \\
& A_{i^{\prime} j^{\prime}}\left(\boldsymbol{\eta}^{n+1,-}\right) \phi_{i^{\prime}}^{n+1,-}=-A_{k j^{\prime}}\left(\boldsymbol{\eta}^{n+1,-}\right) \phi_{k}^{n+1,-} \text {, } \\
& M_{k l} \eta_{l}^{n+1,+}=\frac{4}{3} M_{k l} \eta_{l}^{n+1 / 2}-\frac{1}{3} M_{k l} \eta_{l}^{n,+}+\frac{\Delta t}{3} \frac{\partial H\left(\phi_{i}{ }^{n+1,-}, \eta_{l}{ }^{n+1,-}\right)}{\partial \phi_{k}^{n+1,-}}, \\
& M_{k l} \phi_{k}^{n+1,+}=\frac{4}{3} M_{k l} \phi_{k}^{n+1 / 2}-\frac{1}{3} M_{k l} \phi_{k}^{n,+}-\frac{\Delta t}{3} \frac{\partial H\left(\phi_{i}{ }^{n+1,-}, \eta_{l}{ }^{n+1,-}\right)}{\partial \eta_{l}^{n+1,-}}, \\
& A_{i^{\prime} j^{\prime}}\left(\boldsymbol{\eta}^{n+1,+}\right) \phi_{i^{\prime}}^{n+1,+}=-A_{k j^{\prime}}\left(\boldsymbol{\eta}^{n+1,+}\right) \phi_{k}^{n+1,+} \text {. }
\end{aligned}
$$

Bold symbols here mean that we use the whole range of approximated variables.

We consider now standing waves in a rectangular basin with a unit depth. The basin length $L=10$, the wave number $k=6$, and the maximal free surface amplitude is 
$A=0.25$. The initial conditions of the problem are given as

$$
\eta(x)=0 ; \quad \phi(x, z)=0.075 \cos (k x) \cosh (K z) .
$$

The waves computed with the variational discretization (4.43) show no decay in discrete energy over one hundred periods of time, as can be seen in Fig 4.14. The error in the discrete energy is used to compute the order of accuracy. As we can see in Table 4.4 the scheme is third order accurate in time.

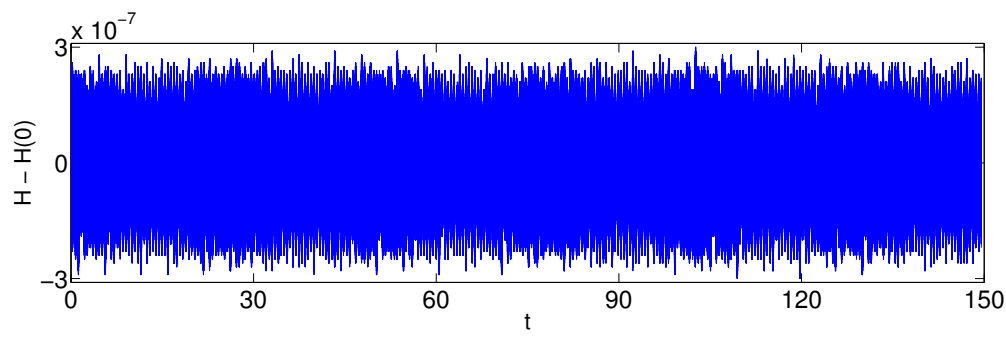

Figure 4.14: Error in the energy for standing waves in a basin after one hundred time periods. The wave number $k=6$, the water depth at rest $H_{0}=1$, and the maximal free surface amplitude is $A=0.25$. Triangular tessellation is used with $N_{x}=81, N_{z}=5$, the time step $\Delta t=0.00625$, precision to solve the implicit subsystem is $\epsilon=10^{-12}$.

\begin{tabular}{l|ll}
\hline \hline Step & $\Delta H$-error & order \\
\hline$\Delta t$ & $2.9372 \mathrm{E}-4$ & - \\
\hline$\Delta t / 2$ & $3.66132 \mathrm{E}-5$ & 3.0040 \\
\hline$\Delta t / 4$ & $4.5574 \mathrm{E}-6$ & 3.0060 \\
\hline$\Delta t / 8$ & $5.6923 \mathrm{E}-7$ & 3.0011 \\
\hline$\Delta t / 16$ & $7.1200 \mathrm{E}-8$ & 2.9991 \\
\hline$\Delta t / 32$ & $8.8996 \mathrm{E}-9$ & 3.0001 \\
\hline$\Delta t / 64$ & $1.1124 \mathrm{E}-9$ & 3.0000 \\
\hline
\end{tabular}

Table 4.4: Convergence of the energy error for standing waves in a square basin using the third order symplectic time integrator 4.43). Triangular tessellation is used with $N_{x}=81, N_{z}=5$, the biggest time step $\Delta t=0.05$, precision to solve the implicit subsystem is $\epsilon=10^{-12}$. The results are compared after ten time periods.

The time integration method (4.43) poses an advantage compared to the second order Störmer-Verlet scheme, presented in this Chapter, see also [52]. The Störmer-Verlet scheme requires a trapezoidal-midpoint quadrature rule, which makes it difficult to find solutions of the Laplace equation in the interior of the domain. The interior velocity potential depends on the free surface elevation and the free surface velocity potential and results in an unsymmetrical approximation of the variables, which is not optimal. The 
surface elevation is approximated in time via $\eta_{s}^{n,+}$ and $\eta_{s}^{n+1,-}$, while the free surface potential is approximated via $\phi_{s}^{n,+}$ and $\phi_{s}^{n+1 / 2}$. The resulting numerical discretization discussed in the first part of this chapter provides excellent results, but also it required a lot of additional analysis. In contrast, the method 4.43 is symmetrical, therefore there is no question how to find the velocity potential in the interior. Moreover, method 4.43 solves one implicit system with three equations, then six explicit steps and gives third order accuracy, while the Störmer-Verlet scheme solves an implicit system for $\phi_{i^{\prime}}, \phi_{s}$ first, then an implicit system for $\eta_{s}, \phi_{i^{\prime}}$ and one explicit step for $\phi_{s}$. Therefore the second order scheme requires the solution of two implicit systems.

\subsection{Conclusions}

A new variational finite element method was presented to compute irrotational water wave dynamics. It was based on a finite element discretization of Miles' variational principle, continuous in space and (dis)continuous Galerkin in time. The key two challenges that have been resolved were:

(i) Dynamic grid motion was taken into account as an integral part of the variational dynamics, with mesh motion in the interior being slaved to the (hyperbolic and Hamiltonian) free surface dynamics.

(ii) Both forced and free wave dynamics of the spatially (non)autonomous Hamiltonian system were discretized in time in a stable manner by direct use of the (spatially discrete) variational principle.

A variational space (plus time) finite element approach was developed, leading to a nonlinear algebraic system. A space-time discontinuous Galerkin finite-element approach will also lead to our discrete variational principle, provided one uses the right basis functions in unison in space and time. This is also a natural framework to explore the geometric conservation law [94] that presumably underpins our discretization implicitly. The methodology will likely work for a fully space discontinuous finite element discretization, as our work on variational DG methods for linear water waves indicates [3]. We used a Newton method to solve the nonlinear algebraic system, in combination with direct solvers available in the PETSc package. The latter gives good performance when the memory for sparse matrix storage is pre-allocated rather than allocated dynamically.

Verification of the numerical discretization against harmonic Fenton waves for 100 time periods demonstrated the accuracy of the novel variational finite element method in simulating waves for a long time. A key advantage of this finite element discretization is that the discrete energy shows no drift in time such that there is no reduction in wave height due to artificial, numerical dissipation. Validation of the numerical method with experimental data reveals that the agreement is good and also shows the robustness of the numerical discretization. Furthermore, the numerical scheme is capable of accurately producing the wave focussing phenomena observed in the experimental data provided by 
the Maritime Institute Netherlands (MARIN). For highly irregular propagating waves the accuracy can be further improved by using a higher order spatial and temporal discretization to obtain a smaller dispersion error.

The numerical validation with the MARIN experiments was somewhat limited because we used reflecting rather than absorbing boundary conditions at the laboratory beach. Interesting variational approaches to solve this boundary issue are: to use a new water wave model we developed [30, 54], which includes the nonlinear water wave limit as well as wave breaking in the form of shallow water bores at the beach, or to employ effective, semi-analytical nonlinear boundary conditions such as developed and used in Kristina et al. [86].

\subsection{Appendix}

\subsubsection{Evaluation of finite element matrices}

In this appendix, we will discuss some aspects of the construction of the finite element matrices. We will assume that the structured mesh with quadrilateral elements is used.

For the evaluation of the matrices in the discrete formulation we first consider the free surface $S_{F}$ of the domain $\Omega_{h}$. The global node number $l=k_{s}(\beta)$ follows from the element $k_{s}$ and local node number $\beta$ via assembly. We compute the Jacobian of the surface element transformation 4.9 as

$$
\bar{J}_{k_{s}}=\frac{\partial x}{\partial \zeta}=x_{k_{s}(1)}(t) \frac{\partial \psi_{1}}{\partial \zeta}+x_{k_{s}(2)}(t) \frac{\partial \psi_{2}}{\partial \zeta}=\frac{\left(x_{k_{s}(2)}(t)-x_{k_{s}(1)}(t)\right)}{2}=\frac{\Delta x_{k_{s}}(t)}{2} .
$$

This relation shows that the Jacobian is time-dependent in the zone where the nodes change due to the wave maker movement. The gradients of the surface basis functions are equal to

$$
\left(\begin{array}{c}
\frac{\partial \varphi_{\beta}}{\partial t} \\
\frac{\partial \varphi_{\beta}}{\partial x}
\end{array}\right)=\frac{1}{\left|\bar{J}_{k_{s}}\right|}\left(\begin{array}{cc}
\frac{\partial x}{\partial \zeta} & -\frac{\partial x}{\partial t} \\
0 & 1
\end{array}\right)\left(\begin{array}{c}
0 \\
\frac{\partial \psi_{\beta}}{\partial \zeta}
\end{array}\right)=\frac{1}{\left|\bar{J}_{k_{s}}\right|}\left(\begin{array}{c}
-\frac{\partial\left(x_{k_{s}(\beta)} \psi_{\beta}\right)}{\partial t} \frac{\partial \psi_{\beta}}{\partial \zeta} \\
\frac{\partial \psi_{\beta}}{\partial \zeta}
\end{array}\right) .
$$

The time derivative of the $x$-coordinate of a free surface node can now be computed using its dependence on the wave maker position 4.11) as

$$
\frac{\partial x_{k_{s}(\beta)}}{\partial t}=\gamma_{x, i_{k_{s}(\beta)}} \frac{\mathrm{d} R}{\mathrm{~d} t} .
$$

This relation shows that the time derivative of the horizontal coordinate is proportional to the wave maker velocity. This allows us to evaluate the time derivative in 4.46 and the matrices $D_{k l}$ and $M_{k l}$ in 4.14. 
For each surface element $\bar{K}_{k_{s}}^{n}$ the contributions to the global matrices $D_{k l}$ and $M_{k l}$ in 4.14) are now computed as follows:

$$
\begin{aligned}
D_{\alpha, \beta} & :=\int_{\bar{K}} \psi_{\beta}\left(\gamma_{x, k_{s}(1)} \psi_{1}+\gamma_{x, k_{s}(2)} \psi_{2}\right) \frac{\partial \psi_{\alpha}}{\partial \zeta} \frac{\mathrm{d} R}{\mathrm{~d} t} \mathrm{~d} \zeta \\
M_{\alpha, \beta} & :=\int_{\bar{K}} \psi_{\alpha} \psi_{\beta}\left|\bar{J}_{k_{s}}\right| \mathrm{d} \zeta .
\end{aligned}
$$

We also define the faces $\bar{K}_{k_{m}}(t)$ on the piston wave maker $S_{W}$, given by the vertical domain $\{R(t)\} \times[-H, \eta]$ and with face number $k_{m}$. Each wave maker face $\bar{K}_{k_{m}}$ is connected to reference element $\bar{K}$ using the transformation $\bar{F}_{w}: \bar{K} \mapsto \bar{K}_{k_{m}}$ defined as

$$
\bar{F}_{w}: \bar{K} \rightarrow \bar{K}_{k_{m}}: \zeta \mapsto z:=z_{k_{m}(\beta)}(t) \psi_{\beta}(\zeta)
$$

where $\beta=1,2$ denote the local node numbers of element $\bar{K}_{k_{m}}$ and the $z_{k_{m}(\beta)}(t)$ 's the coordinates of the vertices of the wave maker face. We compute the Jacobian of the transformation (4.50) at the nodes $l \in \mathcal{N}_{W}$ as

$$
J_{k_{m}}=\frac{\partial z}{\partial \zeta}=z_{k_{m}(1)} \frac{\partial \psi_{1}}{\partial \zeta}+z_{k_{m}(2)} \frac{\partial \psi_{2}}{\partial \zeta}=\frac{1}{2}\left(z_{k_{m}(2)}-z_{k_{m}(1)}\right)=\frac{1}{2} \Delta z_{k_{m}} .
$$

The local contribution per wave maker face $\bar{K}_{k_{m}}$ to the global vector $W_{m}$ in (4.14) has an elemental contribution:

$$
W_{\beta}:=\int_{\bar{K}} \frac{\mathrm{d} R}{\mathrm{~d} t} \psi_{\beta}\left|J_{k_{m}}\right| \mathrm{d} \zeta .
$$

\subsubsection{Variations of the kinetic energy}

The prescribed wave maker motion variations (4.11) of the $x$-coordinates of the nodes are zero: $\delta x_{i_{k}}=\delta\left(x_{i_{k}}(0)+\gamma_{x, i_{k}} R(t)\right)=\gamma_{x, i_{k}} \delta(R(t))=0$. Variations of the $z-$ coordinates do, however, relate to variations of $\eta_{k}$ as

$$
\delta z_{i_{k}}=\delta\left(z_{i_{k}}(0)+\gamma_{z, i_{k}} \eta_{k}\right)=\gamma_{z, i_{k}} \delta \eta_{k}
$$

The Jacobian matrix of the mapping $F_{k_{e}}: \hat{K} \rightarrow K_{k_{e}}$ (i.e., 4.8) with vertex coordinates $\left(x_{k_{e}(\alpha)}, z_{k_{e}(\alpha)}\right)$ is equal to

$$
J_{k_{e}}=\left(\begin{array}{ll}
\frac{\partial x}{\partial \zeta_{1}} & \frac{\partial x}{\partial \zeta_{2}} \\
\frac{\partial z}{\partial \zeta_{1}} & \frac{\partial z}{\partial \zeta_{2}}
\end{array}\right)
$$


Using (4.14, variations of the global matrix $A_{i j}$ for each element $K_{k_{e}}$ are

$$
\begin{aligned}
& \delta_{\eta} A_{\alpha, \beta}(\boldsymbol{\eta}, t)=\delta_{\eta} \int_{K_{k_{e}}}\left(\nabla \tilde{\varphi}_{\alpha} \cdot \nabla \tilde{\varphi}_{\beta}\right) \mathrm{d} x \mathrm{~d} z \\
& =\int_{\hat{K}} \frac{1}{\left|J_{k_{e}}\right|}\left(\begin{array}{l}
\frac{\partial \hat{\psi}_{\alpha}}{\partial \zeta_{1}} \\
\frac{\partial \hat{\psi}_{\alpha}}{\partial \zeta_{2}}
\end{array}\right)^{T}\left[\begin{array}{cr}
2\left(\frac{\partial z}{\partial \zeta_{2}}\right) \delta z_{\zeta_{2}} & -\frac{\partial z}{\partial \zeta_{1}} \delta z_{\zeta_{2}}-\frac{\partial z}{\partial \zeta_{2}} \delta z_{\zeta_{1}} \\
-\frac{\partial z}{\partial \zeta_{1}} \delta z_{\zeta_{2}}-\frac{\partial z}{\partial \zeta_{2}} \delta z_{\zeta_{1}} & 2\left(\frac{\partial z}{\partial \zeta_{1}}\right) \delta z_{\zeta_{1}}
\end{array}\right]\left(\begin{array}{l}
\frac{\partial \hat{\psi}_{\beta}}{\partial \zeta_{1}} \\
\frac{\partial \hat{\psi}_{\beta}}{\partial \zeta_{2}}
\end{array}\right) \\
& \frac{\delta\left|J_{k_{e}}\right|}{\left|J_{k_{e}}\right|^{2}}\left(\begin{array}{l}
\frac{\partial \hat{\psi}_{\alpha}}{\partial \zeta_{1}} \\
\frac{\partial \hat{\psi}_{\alpha}}{\partial \zeta_{2}}
\end{array}\right)^{T}\left[\begin{array}{cr}
\left(\frac{\partial z}{\partial \zeta_{2}}\right)^{2}+\left(\frac{\partial x}{\partial \zeta_{2}}\right)^{2} & -\frac{\partial z}{\partial \zeta_{1}} \frac{\partial z}{\partial \zeta_{2}}-\frac{\partial x}{\partial \zeta_{1}} \frac{\partial x}{\partial \zeta_{2}} \\
-\frac{\partial z}{\partial \zeta_{1}} \frac{\partial z}{\partial \zeta_{2}}-\frac{\partial x}{\partial \zeta_{1}} \frac{\partial x}{\partial \zeta_{2}} & \left(\frac{\partial z}{\partial \zeta_{1}}\right)^{2}+\left(\frac{\partial x}{\partial \zeta_{1}}\right)^{2}
\end{array}\right]\left(\begin{array}{l}
\frac{\partial \hat{\psi}_{\beta}}{\partial \zeta_{1}} \\
\frac{\partial \hat{\psi}_{\beta}}{\partial \zeta_{2}}
\end{array}\right) \mathrm{d} \zeta_{1} \mathrm{~d} \zeta_{2},
\end{aligned}
$$

with $\delta z_{\zeta_{1}}=\delta\left(\partial z / \partial \zeta_{1}\right)$, etc.

Variations of $z$ still depend on the free surface height via 4.11, and by using 4.8) and 4.11) variations of $\delta z_{\zeta_{1}}$ and $\delta z_{\zeta_{2}}$ become

$$
\begin{aligned}
& \delta z_{\zeta_{1}}=-\frac{1-\zeta_{2}}{4} \delta z_{k_{e}(1)}+\frac{1-\zeta_{2}}{4} \delta z_{k_{e}(2)}+\frac{1+\zeta_{2}}{4} \delta z_{j(3)}-\frac{1+\zeta_{2}}{4} \delta z_{k_{e}(4)} \\
& =\left(-\gamma_{z, k_{e}(1)} \frac{1-\zeta_{2}}{4}-\gamma_{z, k_{e}(4)} \frac{1+\zeta_{2}}{4}\right) \delta \eta_{k}+\left(\gamma_{z, k_{e}(2)} \frac{1-\zeta_{2}}{4}+\gamma_{z, k_{e}(3)} \frac{1+\zeta_{2}}{4}\right) \delta \eta_{k+1} \\
& \delta z_{\zeta_{2}}=-\frac{1-\zeta_{1}}{4} \delta z_{k_{e}(1)}-\frac{1+\zeta_{1}}{4} \delta z_{k_{e}(2)}+\frac{1+\zeta_{1}}{4} \delta z_{k_{e}(3)}+\frac{1-\zeta_{1}}{4} \delta z_{k_{e}(4)} \\
& =\left(\gamma_{z, k_{e}(4)} \frac{1-\zeta_{1}}{4}-\gamma_{z, k_{e}(1)} \frac{1-\zeta_{1}}{4}\right) \delta \eta_{k}+\left(\gamma_{z, k_{e}(3)} \frac{1+\zeta_{1}}{4}-\gamma_{z, k_{e}(2)} \frac{1+\zeta_{1}}{4}\right) \delta \eta_{k+1} .
\end{aligned}
$$

Note that we aligned the first and fourth vertices of the element $K_{k_{e}}$ to be on the same mesh column as the corresponding free surface node $k$. Similarly, the second and third element vertex are at the same column as the free surface node $k+1$. The crucial part of these relations is that variations in the free surface are coupled to variations in the mesh. This dependence cannot be ignored in computing the variations of the discrete Miles' functional. Otherwise, incorrect dynamical equations are obtained, unstable for large amplitude wave motions. Finally, the variation of the Jacobian is given by

$$
\delta\left|J_{k_{e}}\right|=\left(\partial x / \partial \zeta_{1}\right) \delta z_{\zeta_{2}}-\left(\partial x / \partial \zeta_{2}\right) \delta z_{\zeta_{1}} .
$$

After substitution of 4.55 and 4.56 into 4.54, we can assemble $C_{k i j}$ in 4.33. 
Similarly, contributions of the face $\bar{K}_{k_{m}}$ at the wave maker to the variation of $W_{m}$ with respect to wave height $\eta$ are equal to

$$
\delta_{\eta} W_{\alpha}\left(\eta_{q}, t\right)=\delta_{\eta} \int_{\bar{K}_{k_{m}}} \frac{\mathrm{d} R}{\mathrm{~d} t} \varphi_{\alpha} \mathrm{d} z=\int_{\bar{K}} \frac{\mathrm{d} R}{\mathrm{~d} t} \psi_{\alpha} \delta\left|J_{k_{m}}\right| \mathrm{d} \zeta_{2},
$$

where $m=k_{m}(\alpha)$ is the global node number, and the variations of the Jacobian $\left|J_{k_{m}}\right|$ are given by

$$
\delta_{\eta}\left|J_{k_{m}}\right|=\delta_{\eta}\left(\frac{1}{2}\left(z_{k_{m}(2)}-z_{k_{m}(1)}\right)\right)=\frac{1}{2}\left(\gamma_{z, k_{m}(2)}-\gamma_{z, k_{m}(1)}\right) \delta \eta_{q},
$$

with $q \in \mathcal{N}_{F} \cap \mathcal{N}_{W}$. Finally, substituting (4.58) into 4.57) allows assembly of contributions in 4.35. 


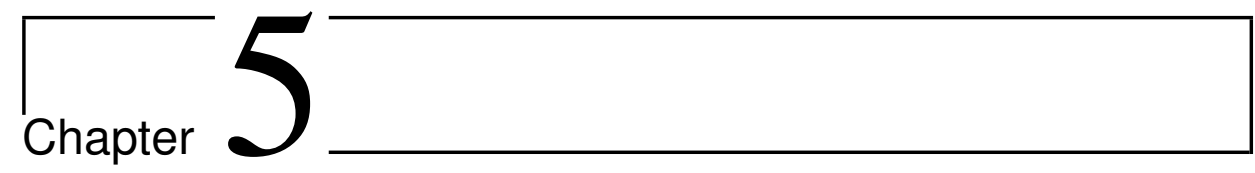

\section{Conclusions}

In this thesis various aspects of water wave modelling are considered. The main topic is the development of an efficient numerical method to construct a numerical wave tank using a variational approach. This allows the creation of stable numerical methods methods with no energy drift and bounded oscillations, which are based on a mixed space continuous - time discontinuous Galerkin finite element method. The resulting scheme was verified against the semi-analytical solution of nonlinear potential flow water waves derived by Fenton and Rienecker, [47]. An extensive comparison with laboratory data provided by MARIN was also performed.

In this thesis a number of theoretical and practical aspects had to be considered. Here, we summarize the main steps, results; and, suggest directions for future work.

\subsection{Recommendations}

An interesting future extension of the research presented in this thesis will be to obtain a linear shallow water solution for a run up in the basin set up analogous to the one used in Bore-Soliton-Splash experiment. A number of solutions for various bathimetries was already considered in [118]. It is also interesting to determine the amplification factor for the simplest analytical model. One of the reasons to explore this is the fact that quite some early tsunami warning systems use the linear shallow water equations for the run up simulation. Many bays along the Pacific coast of Japan have an inclined bottom. It would be interesting, therefore, to make a run up analogy of the Bore-SolitonSplash experiment for realistic bathimetry. Nevertheless, it is worth to mention that the Bore-Soliton-Splash is a nonlinear phenomena. A collision of solitons that results in a massively amplified wave was analytically described for weakly nonlinear flow with the Kadomtsev-Petviashvilli equations by Y. Kodama in 2012 following the method described in [157]. 
A further investigation of the jump conditions presented in Chapter 2 is required. It was not studied analytically whether the obtained time derivative of the energy functional during the "wave breaking" in the potential flow system is sign-definite. One could verify this numerically by simulating the wave phenomena when a barrier between two domains with different water height is removed.

The time integrator theme leaves a broad range of possible extensions. We haven't tried fourth and higher order polynomials yet. The third order scheme was verified only for an autonomous case. Can it handle non-autonomous problems, such as waves generated by a wave maker?

For the numerical wave tank, naturally, it is necessary to extend the code to three dimensions, which is ongoing work. The inclusion of (a vertical component of) vorticity is also an important extension. Other model extensions would be adaptive mesh refinement at locations with strong wave interaction, such as in the splash zone of a freak wave. Adaptive time stepping will be necessary to save computing time, nevertheless in order to keep the symplectic structure a further analysis has to be performed. The modeling of a beach could also be included. The variational coupling technique, investigated by Kristina, [86], [87], [85], and F. Klaver [81], provides a very useful approach. In the current model one could couple the potential flow code for "deep" water in a similar way with an analytical solution, for example, at the shore. The laboratory experiments of Chabchoub, [24], provide a nice data set for validation of our numerical method. This comparison is fairly easy to do - one just needs to take the appropriate basin set up. As it could be observed in [53] an adaptive mesh technique coupled with the elliptic mesh generator of Knupp [83], has enabled us to get stable results for fairly nonlinear waves with a height equal to the basin depth. This adaptive meshing technique was not used so far for the piston wave maker case, since it is very costly, but improvements in its efficiency are certainly possible. 


\section{Summary}

This thesis starts in Chapter 2 with the study the theoretical aspects of water wave modelling using a variational framework, which is directly associated with phase space and energy conservation laws. In particular, we focus on a new variational model [54] based on the work of Cotter and Bokhove, [30]. This model includes a potential flow model, the shallow water model and the Boussinesq type model of Klopman [82] as its limits. The new model embraces the benefits of the mentioned models, such as accurate dispersion and the incorporation of the vertical component of vorticity and breaking waves modeled as bores. In this thesis the Hamiltonian structure of the new water wave model is derived. A novel approach to find jump conditions at a bore is presented based on the work of Wakelin [148]. This allows the recovery of known results for bores within the shallow water equations. The question of stability of the solution in the bore still remains uninvestigated.

In general, preserving the properties of physical and mathematical systems at the discrete level is desirable since in general it may result in superior numerical discretizations. The Hamiltonian framework provides a powerful approach to analyze the conservation properties and stability of some nonlinear (fluid) systems. In addition, symplectic time integrators are suitable to integrate Hamiltonian systems in time and preserve many important properties of these systems after time discretization. In Chapter 3 we use a variational approach to derive a class of symplectic time integrators. We start with a Hamiltonian system with coordinate and momentum as canonically conjugated variables. A discrete variational principle in time is constructed, which is discretized with a discontinuous Galerkin finite element method. To connect disjoint time elements a specially chosen numerical flux is used, which is associated with the work of Rhebergen et al. [125], [126], and Dal Maso, Le Floch and Murat [34] on nonconservative products. Following this approach we are able to obtain known time integrators, such as the symplectic Euler, symplectic modified midpoint and Störmer-Verlet schemes. Some new time integration schemes are obtained as well. The variational approach gives some freedom in the choices of parameters. Naturally, not every choice leads to a stable integrator with ex- 
cellent dispersion properties. We extend therefore an existing analysis to make it suitable for the time discontinuous variational discretization.

The next topic considered in this thesis is the construction of a numerical wave tank, which is discussed in Chapter 4 A second order accurate variational finite element method for the nonlinear potential flow water wave equations is developed. The potential flow equations include dispersion, which is important in modelling waves of intermediate depth generated by a wave maker. An important aspect is that the free surface and wave maker motion changes the computational domain each time step. The verification of the numerical method includes a comparison with the semi-analytical solution of Fenton and Rienecker [47], which shows an absence of energy drift for long time simulations. A detailed validation using experimental wave tank data demonstrates the excellent capabilities of the numerical method to simulate realistic wave experiments. In particular, wave focussing is simulated accurately, catching both the location and time when a freak wave occurred in the experiment. Also, the numerical spectra coincide well with the measured spectra of the incoming waves at a number of wave probes. The results for an uneven bottom and irregular waves are also found to be excellent. These results provide a suitable basis for the extension of the method to a fast moving ship in waves.

In Chapter 4 we also combine the novel third order time integrator developed in Chapter 3 with the discrete variational method constructed earlier in Chapter 4 for nonlinear free surface water waves. The resulting numerical approximation is verified against a test case of standing waves in a wave basin with solid walls. A long time calculation reveals no energy or amplitude decay, which provides an adequate base for a further usage of the third order time integrator for more complicated water wave problems. 


\section{Samenvatting}

Als eerste onderwerp van dit proefschrift worden in hoofdstuk 2 de theoretische aspecten van het modelleren van watergolven besproken. Hiervoor wordt een variationele aanpak gebruikt die gerelateerd is aan de faseruimte en de wet van behoud van energie. We hebben daarbij een nieuw variationeel model [54] bestudeerd dat gebaseerd is op het werk van Cotter en Bokhove, [30]. Dit model beschrijft als bijzondere gevallen onder andere een model voor watergolven in een potentiaalstroming, een model voor watergolven op ondiep water en een Boussinesq model dat ontwikkeld is door Klopman [82]. Dit nieuwe watergolfmodel combineert de voordelen van genoemde modellen, zoals bijvoorbeeld nauwkeurige dispersie eigenschappen, de beschrijving van een verticale component van de vorticiteit, en brekende golven die gemodelleerd worden als een discontinue sprong in de waterhoogte ("bore"). In dit proefschrift wordt de Hamiltoniaanse structuur van dit nieuwe watergolfmodel afgeleid. Daarnaast wordt een nieuwe aanpak besproken om sprong relaties in het model voor een "bore" af te leiden. Deze method is gebaseerd op het werk van Wakelin [148]. Met dit model zijn bekende resultaten voor modellen voor brekende golven in ondiep water verkregen. Er is niet gekeken naar de stabiliteit van de oplossing van een "bore".

In het algemeen is het wenselijk om de eigenschappen van zowel de fysieke als wiskundige systemen op discreet niveau te behouden, aangezien dit in het algemeen leidt tot een betere numerieke discretisatie. Het Hamiltoniaanse kader biedt een krachtige methode om de behoudswetten en stabiliteit van sommige niet-lineaire (vloeistof)systemen te analyseren. Bovendien kunnen symplectische tijdsintegratiemethoden, die veel belangrijke eigenschappen van het systeem op discreet niveau behouden, gebruikt worden om Hamiltoniaanse systemen in de tijd te integreren. In hoofdstuk 3 gebruiken we een variationele methode om een klasse van symplectische tijdsintegratiemethoden af te leiden. We beginnen met een Hamiltoniaans systeem met de positie en moment als canonieke geconjugeerde variabelen. Een discreet variationeel principe in de tijd is geconstrueerd, waarna dit wordt gediscretiseerd met een discontinue Galerkin eindige elementen methode. Om de disjuncte tijdselementen aan elkaar te koppelen wordt er gebruik gemaakt 
van een speciaal gekozen numerieke flux. Deze numerieke flux is geassocieerd met het onderzoek naar niet-conservatieve producten van Rhebergen et. al. [125], [126] en Dal Maso, Le Floch en Murat [34]. Met deze aanpak waren we in staat om bekende tijdsintegratiemethoden af te leiden, zoals het symplectische Euler schema, het symplectische gemodificeerde middelpunt schema en het Störmer-Verlet schema. Verder zijn er enkele nieuwe tijdsintegratiemethoden verkregen. Binnen het variationele kader is er nog enige ruimte in de keuze van parameters. Niet elke keuze van parameters leidt tot een stabiele tijdsintegratiemethode met de gewenste dispersie eigenschappen. Daarom breiden we de bestaande analyse uit om deze geschikter te maken voor discontinue variationele discretizaties in de tijd.

Het volgende onderwerp dat bestudeerd wordt in dit proefschrift is de ontwikkeling van een numeriek model voor het beschrijven van watergolven in een golfbasin. Dit wordt besproken in hoofdstuk 4 In dit hoofdstuk wordt een tweede orde nauwkeurige variationele eindige elementenmethode ontwikkeld voor watergolven in een potentiaalstroming. De vergelijking voor watergolven in een potentiaalstroming beschrijft het dispersiegedrag van de watergolven correct, wat belangrijk is voor het modelleren van golven die gegenereerd worden door een golfopwekker op ondiep water. Een belangrijk aspect hierbij is dat door de beweging van het vrije oppervlak en de golfopwekker het domein waarop de berekeningen worden uitgevoerd ieder tijdstap verandert.

De verificatie van de numerieke methode bestaat onder andere uit een vergelijking met de semi-analytische oplossing van Fenton en Rienecker[47]. Deze berekeningen laten zien dat de totale hoeveelheid energie behouden blijft bij het simuleren over een lange tijdspanne. Een gedetailleerde validatie, die gebruikt maakt van experimenten in een golftank, laat ook zien dat de numerieke methode realistische golfexperimenten nauwkeurig kan simuleren. In het bijzonder het focusseren van de golven wordt accuraat gesimuleerd. Hierbij worden zowel de plaats als de tijd goed voorspeld wanneer er een extreme golf werd waargenomen in de experimenten. Bovendien komen de numerieke spectra voor inkomende golven goed overeen met de spectra die gemeten werden met een aantal golfharken. De resultaten voor een oneffen bodem en onregelmatige golven zijn ook uitstekend. Deze resultaten vormen een geschikte basis voor de uitbreiding van de methode om een snel schip in golven te simuleren.

Tot slot combineren we in hoofdstuk 4 de nieuwe derde orde tijdsintegratiemethode, die ontwikkeld is in hoofdstuk 3 , met de variationele methode voor niet-lineaire watergolven die in het eerste deel van hoofdstuk 4 is beschreven. De resulterende numerieke oplossing is geverifieerd met behulp van een berekening voor staande golven in een golftank met vaste wanden. Het simuleren over een lange tijd laat zien dat er geen sprake is van een afname in de energie of de amplitude van de watergolven. De nieuwe derde orde nauwkeurige tijdsintegratie methode geeft daarmee een goede basis voor berekeningen aan meer complexe watergolfproblemen. 


\section{Bibliography}

[1] D. Adytia. Coastal zone simulations with variational Boussinesq modelling. $\mathrm{PhD}$ thesis, University of Twente, Enschede, May 2012.

[2] A. Ali and H. Kalisch. Energy balance for undular bores. Comptes Rendus Mecanique, 338(2):67 - 70, 2010.

[3] V. R. Ambati. Forecasting Water Waves and Currents: A Space-time Approach. $\mathrm{PhD}$ thesis, University of Twente, Enschede, February 2008.

[4] M. Antuono. A shock solution for the nonlinear shallow water equations. Journal of Fluid Mechanics, 658:166-187, 92010.

[5] D. N. Arnold, F. Brezzi, B. Cockburn, and L. Donatella Marini. Unified analysis of discontinuous Galerkin methods for elliptic problems. SIAM Journal on Numerical Analysis, pages 1749-1779, 2001.

[6] V. I. Arnold. Mathematical Methods of Classical Mechanics, volume 60 of Graduate Texts in Mathematics. Springer, 1989. Originally published by Nauka, Moscow, 1974, 2nd ed. 1989, XVI, 519 p.

[7] J. Bajars. Geometric integration and thermostat methods for Hamiltonian systems. $\mathrm{PhD}$ thesis, University of Amsterdam (UvA), Amsterdam, December 2012.

[8] H. Bateman. Partial Differential Equations of Mathematical Physics. Cambridge University Press, 2nd edition, 1944.

[9] J. T. Beale. A convergent boundary integral method for three-dimensional water waves. Mathematics of Computation, 70:977-1029, 2001.

[10] T. B. Benjamin and P. J. Olver. Hamiltonian structure, symmetries and conservation laws for water waves. Journal of Fluid Mechanics, 125:137-185, 121982. 
[11] P. Bettess. Operation counts for boundary integral and finite element methods. International Journal for Numerical Methods in Engineering, 17(2):306-308, 1981.

[12] E. M. Bitner-Gregersen, L. I. Eide, T. Hørte, and R. Skjong. Ship and Offshore Structure Design in Climate Change Perspective. SpringerBriefs in Climate Studies, XII. Springer, 2013.

[13] O. Bokhove, E. Gagarina, W. Zweers, and A. Thornton. Bore-soliton-splash: van spektakel naar oceaangolf. Nederlands Tijdschrift voor Natuurkunde, 77:450-454, 2011.

[14] P. Bonneton, E. Barthelemy, F. Chazel, R. Cienfuegos, D. Lannes, F. Marche, and M. Tissier. Recent advances in Serre-Green-Naghdi modelling for wave transformation, breaking and runup processes. European Journal of Mechanics - B/Fluids, 30(6):589 - 597, 2011. Special Issue: Nearshore Hydrodynamics.

[15] A.-C. Boulanger, M.-O. Bristeau, and J. Sainte-Marie. Analytical solutions for the free surface hydrostatic Euler equations. Communications in Mathematical Sciences, 11(4):993 - 1010, 2013.

[16] J. Boussinesq. Théorie de l'intumescence liquide, applellée onde solitaire ou de translation, se propageant dans un canal rectangulaire. Comptes Rendus de l'Academie des Sciences, 72:755-759, 1871.

[17] T. J. Bridges and N. M. Donaldson. Variational principles for water waves from the viewpoint of a time-dependent moving mesh. Mathematika, 57:147-173, 12011.

[18] T. J. Bridges and D. J. Needham. Breakdown of the shallow water equations due to growth of the horizontal vorticity. Journal of Fluid Mechanics, 679:655-666, 7 2011.

[19] M. J. Briggs, C. E. Synolakis, G. S. Harkins, and D. R. Green. Laboratory experiments of tsunami runup on a circular island. Pure and applied geophysics, 144(3-4):569-593, 1995.

[20] L. J. F. Broer. On the Hamiltonian theory of surface waves. Applied Scientific Research, 29(1):430-446, 1974.

[21] J. Broeze, E. F. G. van Daalen, and P. J. Zandbergen. A three-dimensional panel method for nonlinear free surface waves on vector computers. Computational Mechanics, 13(1-2):12-28, 1993.

[22] T. Bunnik. Benchmark workshop on numerical wave modeling - description of test cases. Technical Report 700221RD, MARIN, The Netherlands, Wageningen, The Netherlands, 2010. 
[23] H. C. A. A. Carbonel, A. C. Galeãpoundso, and A. D. Loula. Numerical study of Petrov-Galerkin formulations for the shallow water wave equations. Journal of the Brazilian Society of Mechanical Sciences, 22:231 - 247, 002000.

[24] A. Chabchoub, N. P. Hoffmann, and N. Akhmediev. Rogue wave observation in a water wave tank. Physical Review Letters, 106:204502, May 2011.

[25] J. Chambarel, C. Kharif, and O. Kimmoun. Generation of two-dimensional steep water waves on finite depth with and without wind. European Journal of Mechanics - B/Fluids, 29(2):132 - 142, 2010.

[26] A. Chawla and J. T. Kirby. Experimental study of breaking waves on a blocking current. In Proc. 26th International Conference on Coastal Engineering, Copenhagen, volume 5 of Ocean Engineering, pages 759-772, June 1998.

[27] D. Clamond and D. Dutykh. Practical use of variational principles for modeling water waves. Physica D: Nonlinear Phenomena, 241(1):25 - 36, 2012.

[28] G. F. Clauss, C. E. Schmittner, and J. Hennig. Systematically varied rogue wave sequences for the experimental investigation of extreme structure behavior. Journal of Offshore Mechanics and Arctic Engineering, 130(2):11, 2008.

[29] J. W. Clebsch. Ueber die integration der hydrodynamischen gleichungen. Journal Fur Die Reine Und Angewandte Mathematik, 56, 1859.

[30] C. Cotter and O. Bokhove. Variational water-wave model with accurate dispersion and vertical vorticity. Journal of Engineering Mathematics, 67(1-2):33-54, 2010.

[31] W. Craig and M. D. Groves. Hamiltonian long-wave approximations to the waterwave problem. Wave Motion, 19(4):367 - 389, 1994.

[32] W. Craig and C. Sulem. Numerical simulation of gravity waves. Journal of Computational Physics, 108(1):73 - 83, 1993.

[33] A. D. D. Craik. The origins of water wave theory. Annual Review of Fluid Mechanics, 36(1):1-28, 2004.

[34] G. Dal Maso, P. G. LeFloch, and F. Murat. Definition and weak stability of nonconservative products. Journal de Math/ematiques Pures et Appliqu/ees, 74:483-548, 1995.

[35] I. I. Daniljuk. On integral functionals with a variable domain of integration. Proceedings of the Steklov Institute of Mathematics, 118:1-144, 1976.

[36] O. Darrigol. The spirited horse, the engineer, and the mathematician: Water waves in nineteenth-century hydrodynamics. Archive for History of Exact Sciences, 58(1):21-95, 2003. 
[37] R. G. Dean and R. A. Dalrymple. Water wave mechanics for engineers and scientists, volume 2 of Advanced series on ocean engineering. Singapore [etc.]: World Scientific, 1991.

[38] M. Delfour, W. Hager, and F. Trochu. Discontinuous Galerkin methods for ordinary differential equations. Mathematics of Computation, 36(154):pp. 455-473, 1981.

[39] M. C. Delfour and F. Dubeau. Discontinuous polynomial approximations in the theory of one-step, hybrid and multistep methods for nonlinear ordinary differential equations. Mathematics of Computation, 47(175):pp. 169-189, 1986.

[40] F. Dias and T. J. Bridges. The numerical computation of freely propagating timedependent irrotational water waves. Fluid Dynamics Research, 38(12):803, 2006.

[41] R. Eatock-Taylor, B. T. Wang, and G. X. Wu. On the transient analysis of the wavemaker. In Proc. 9th International Workshop on Water Waves and Floating Bodies, Kuju, Oita, Japan, 1994.

[42] G. A. El, R. H. J. Grimshaw, and N. F. Smyth. Unsteady undular bores in fully nonlinear shallow-water theory. Physics of Fluids (1994-present), 18(2), 2006.

[43] F. Enet and S. T. Grilli. Experimental study of tsunami generation by threedimensional rigid underwater landslides. Journal of Waterway, Port, Coastal, and Ocean Engineering, 133:442, 2007.

[44] D. Estep and D. French. Global error control for the continuous Galerkin finite element method for ordinary differential equations. ESAIM: Mathematical Modelling and Numerical Analysis, 28:815-852, 1994.

[45] C. R. Ethier and D. A. Steinman. Exact fully 3D Navier-Stokes solutions for benchmarking. International Journal for Numerical Methods in Fluids, 19:369-375, Sept. 1994.

[46] O. M. Faltinsen. Numerical solutions of transient nonlinear free surface motion outside of inside or inside moving bodies. In Proc. 2nd Int. Conf. Num. Ship Hydrodyn, Berkley, CA, volume 15, pages 347-357, Apr. 1977.

[47] J. D. Fenton and M. M. Rienecker. A Fourier method for solving nonlinear waterwave problems: application to solitary-wave interactions. Journal of Fluid Mechanics, 118:411-443, 1982.

[48] B. A. Finalson. The method of weighted residuals and variational principles. Academic press, 1972.

[49] H. Flanders. Differentiation under the integral sign. The American Mathematical Monthly, 80(6):615-627, 1973. 
[50] C. F. Fochesato and F. Dias. A fast method for nonlinear three-dimensional freesurface waves. Proceedings of the Royal Society A, 462(2073):2715-2735, 2006.

[51] C. F. Fochesato, S. T. Grill, and F. Dias. Numerical modeling of extreme rogue waves generated by directional energy focusing. Wave Motion, 44:395-416, 2007.

[52] E. Gagarina, V. R. Ambati, J. J. W. van der Vegt, and O. Bokhove. Variational space-time (dis)continuous Galerkin method for nonlinear free surface water waves. Journal of Computational Physics, 275(0):459 - 483, 2014.

[53] E. Gagarina, J. J. W. van der Vegt, V. R. Ambati, and O. Bokhove. A Hamiltonian Boussinesq model with horizontally sheared currents. In Proc. 3rd Int. Symp. on Shallow Flows, Iowa, USA, June 2012.

[54] E. Gagarina, J. J. W. van der Vegt, and O. Bokhove. Horizontal circulation and jumps in Hamiltonian wave models. Nonlinear Processes in Geophysics, 20(4):483-500, 2013.

[55] A. W. Gill and W. W. Read. Efficient analytic series solutions for two-dimensional potential flow problems. International Journal for Numerical Methods in Fluids, 23(5):415-430, 1996.

[56] K. Goto, T. Kawana, and F. Imamura. Historical and geological evidence of boulders deposited by tsunamis, southern Ryukyu Islands, Japan. Earth-Science Reviews, 102(1-2):77 - 99, 2010.

[57] S. Gottlieb, G. W. Wei, and S. Zhao. A unified discontinuous Galerkin framework for time integration, June 2010.

[58] S. T. Grilli, P. Guyenne, and F. Dias. A fully non-linear model for threedimensional overturning waves over an arbitrary bottom. International Journal for Numerical Methods in Fluids, 35(7):829-867, 2001.

[59] R. Grimshaw. Wave action and wave-mean flow interaction, with application to stratified shear flows. Annual Review of Fluid Mechanics, 16(1):11-44, 1984.

[60] E. W. C. v. Groesen, T. Bunnik, and A. Andonowati. Surface wave modelling and simulation for wave tanks and coastal areas. In Proceedings of the International Conference on Developments in Marine CFD, Chennai, India, International Conference on Developments in Marine CFD, pages 1-5, London, 2011. The Royal Institution of Naval Architects.

[61] M. Gross. Conserving Time Integrators for Nonlinear Elastodynamics. PhD thesis, Department of Mechanical Engineering, University of Kaiserslautern, 2004.

[62] E. Guerber, M. Benoit, S. T. Grilli, and C. Buvat. A fully nonlinear implicit model for wave interactions with submerged structures in forced or free motion. Engineering Analysis with Boundary Elements, 36(7):1151 - 1163, 2012. 
[63] P. Guyenne and S. T. Grilli. Numerical study of three-dimensional overturning waves in shallow water. Journal of Fluid Mechanics, 547:361-388, 2006.

[64] E. Hairer, C. Lubich, and G. Wanner. Geometric numerical integration. Springer series in computational mathematics. Berlin: Springer, 2nd edition, 2006.

[65] J. Hall and M. Leok. Spectral Variational Integrators. ArXiv e-prints, Nov. 2012.

[66] R. Hargreaves. A pressure-integral as kinetic potential. Philosophical Magazine, 6:436-444, 1908.

[67] M. He, B. Ren, F. Jiang, and C. Ma. Simulation of Dynamic Coupling Between Waves and a Free-Floating Rectangular Box by Smoothed Particle Hydrodynamics. In Proc. ASME 2013 32nd International Conference on Ocean, Offshore and Arctic Engineering, Nantes, France, volume 5 of Ocean Engineering, June 2013.

[68] J. Hennig and C. E. Schmittner. Experimental Variation of Focusing Wave Groups for the Investigation of Their Predictability. In Proc. ASME 2009 28th International Conference on Ocean, Offshore and Arctic Engineering, Honolulu, Hawaii, USA, volume 6 of Materials Technology; C.C. Mei Symposium on Wave Mechanics and Hydrodynamics; Offshore Measurement and Data Interpretation, page 11, June 2009.

[69] T. Hou and P. Zhang. Convergence of a boundary integral method for 3D water waves. SIAM Journal on Numerical Analysis, 2:1-34, 2002.

[70] R. H. M. Huijsmans, G. Klopman, N. Karjanto, and A. Andonowati. Experiments on extreme wave generation using the Soliton on Finite Background. ArXiv eprints, Oct. 2011.

[71] A. Jensen, G. K. Pedersen, and D. J. Wood. An experimental study of wave run-up at a steep beach. Journal of Fluid Mechanics, 486:161-188, July 2003.

[72] R. S. Johnson, editor. A modern introduction to the mathematical theory of water waves. Cambridge University Press, 1997.

[73] R. S. Johnson, editor. The Handbook of Fluid Dynamics. CRC Pres, 1998.

[74] E. Kalnay. Atmospheric Modeling, Data Assimilation and Predictability. Cambridge University Press, 2003.

[75] O. Karakashian and C. Makridakis. A space-time finite element method for the nonlinear Schrödinger equation: The continuous Galerkin method. SIAM Journal on Numerical Analysis, 36(6):1779-1807, 1999.

[76] C. Kharif, E. N. Pelinovsky, and A. Slunyaev. Rogue Waves in the Ocean. Springer, 2009. 
[77] C. Kim, A. Clement, and K. Tanizawa. Recent research and development of numerical wave tanks-a review. International Journal of Offshore and Polar Engineering, 9:241-256, 1999.

[78] J. W. Kim and K. J. Bai. A finite element method for two-dimensional water-wave problems. International Journal for Numerical Methods in Fluids, 30(1):105-122, 1999.

[79] J. W. Kim, K. J. Bai, R. C. Ertekin, and W. C. Webster. A strongly-nonlinear model for water waves in water of variable depth - the irrotational Green-Naghdi model. Journal of Offshore Mechanics and Arctic Engineering, 125:25-32, 2003.

[80] O. Kimmoun, H. Branger, and B. Zucchini. Laboratory PIV measurements of wave breaking on a beach. In Offshore and Polar Engineering Conference, Toulon, France, Ocean Engineering, May 2004.

[81] F. Klaver. Coupling of numerical models for deep and shallow water. Master's thesis, University of Twente, the Netherlands, 2009.

[82] G. Klopman. Variational Boussinesq modelling of surface gravity waves over bathymetry. PhD thesis, University of Twente, Enschede, The Netherlands, May 2010.

[83] P. M. Knupp. A robust elliptic grid generator. Journal of Computational Physics, 100(2):409-418, June 1992.

[84] J. Kozák and V. Cermák. The Illustrated History of Natural Disasters. SpringerVerlag, 2010.

[85] W. Kristina. Effective Coastal Boundary Conditions for Tsunami Propagation. $\mathrm{PhD}$ thesis, University of Twente, Enschede, The Netherlands, 2014.

[86] W. Kristina, O. Bokhove, and E. W. C. van Groesen. Effective coastal boundary conditions for tsunami wave run-up over sloping bathymetry. Nonlinear Processes in Geophysics Discussions, 1(1):317-369, 2014.

[87] W. Kristina, E. W. C. van Groesen, and O. Bokhove. Effective coastal boundary conditions for dispersive tsunami propagation. Revision Theor. Comput. Fluid Dynamics, page 20, 2013. Submitted July 2012.

[88] E. A. Kuznetsov and A. V. Mikhailov. On the topological meaning of canonical Clebsch variables. Physics Letters A, 77(1):37 - 38, 1980.

[89] I. Lakhturov, D. Adytia, and E. W. C. van Groesen. Optimized variational 1D Boussinesq modelling for broad-band waves over flat bottom. Wave motion, 49(2):309-322, March 2012. 
[90] L. D. Landau and E. M. Lifshitz. Fluid Mechanics, volume 6. ButterworthHeinemann, 2nd edition, 1987.

[91] A. L. Latifah and E. W. C. v. Groesen. Coherence and predictability of extreme events in irregular waves. Nonlinear Processes in Geophysics, 19(2):199-213, 2012.

[92] B. Leimkuhler and S. Reich. Simulating Hamiltonian dynamics, volume 14. Cambridge University Press, 2004.

[93] M. Leok and T. Shingel. General Techniques for Constructing Variational Integrators. ArXiv e-prints, Feb. 2011.

[94] M. Lesoinne and C. Farhat. Geometric conservation laws for flow problems with moving boundaries and deformable meshes, and their impact on aeroelastic computations. Computer Methods in Applied Mechanics and Engineering, 134(12):71 $-90,1996$.

[95] R. Levi. The wave that changed science, Mar. 2008. http://thefutureofthings.com/3698-the-wave-that-changed-science.

[96] C. C. Lin. Liquid Helium. In Proc. of the Enrico Fermi International School of Physics, Course XXI, New York. Academic Press, 1953.

[97] M. S. Longuet-Higgins and E. D. Cokelet. The deformation of steep surface waves on water. I. A numerical method of computation. Proceedings of the Royal Society of London. Series A, Mathematical and Physical Sciences, 350(1660):pp. 1-26, 1976.

[98] J. C. Luke. A variational principle for a fluid with a free surface. Journal of Fluid Mechanics, 27(2):395-397, 1967.

[99] Q. W. Ma, G. X. Wu, and R. Eatock-Taylor. Finite element simulation of fully nonlinear interaction between vertical cylinders and steep waves. part 1: methodology and numerical procedure. International Journal for Numerical Methods in Fluids, 36(3):265-285, 2001.

[100] Q. W. Ma, G. X. Wu, and R. Eatock-Taylor. Finite element simulations of fully non-linear interaction between vertical cylinders and steep waves. part 2: numerical results and validation. International Journal for Numerical Methods in Fluids, 36(3):287-308, 2001.

[101] Q. W. Ma and S. Yan. Quasi $\{$ ALE $\}$ finite element method for nonlinear water waves. Journal of Computational Physics, 212(1):52 - 72, 2006.

[102] N. I. Makarenko and V. K. Kostikov. Non-linear water waves generated by impulsive motion of submerged obstacle. Natural Hazards and Earth System Sciences Discussions, 1(6):7647-7665, 2013. 
[103] J. E. Marsden and T. S. Ratiu. Introduction to mechanics and symmetry: a basic exposition of classical mechanical systems, volume 17. Springer, 1999.

[104] J. E. Marsden and M. West. Discrete mechanics and variational integrators. Acta Numerica 2001, 10:357-514, 52001.

[105] D. M. Milder. A note on: "on Hamilton's principle for surface waves". Journal of Fluid Mechanics, 83:159-161, 1977.

[106] J. W. Miles. On Hamilton's principle for surface waves. Journal of Fluid Mechanics, 83:153-158, 111977.

[107] J. W. Miles and R. Salmon. Weakly dispersive nonlinear gravity waves. Journal of Fluid Mechanics, 157:519-531, 1985.

[108] F. Mohammed, B. McFall, and H. M. Fritz. Tsunami Generation by 3D Deformable Granular Landslides, chapter 28, pages 310-320. American Society of Civil Engineers, 2011.

[109] P. J. Morrison. Hamiltonian description of the ideal fluid. Rev. Mod. Phys., 70:467521, Apr 1998.

[110] I. Nikolkina and I. Didenkulova. Catalogue of rogue waves reported in media in 2006-2010. Natural Hazards, 61(3):989-1006, 2012.

[111] E. Noether. Invariant variation problems. Transport Theory and Statistical Physics, 1:186-207, 1971.

[112] S. Nurijanyan. Discrete and continuous Hamiltonian systems for wave modelling. $\mathrm{PhD}$ thesis, University of Twente, Enschede, October 2013.

[113] S. Ober-Blöbaum and N. Saake. Construction and analysis of higher order Galerkin variational integrators. ArXiv e-prints, Apr. 2013.

[114] E. Okal, C. de Beer, J. Visser, and N. Kalligeris. The rogue wave of 27 August 1969 at Dwarskersbos, South Africa: Field survey and simulation as a meteo-tsunami. In EGU General Assembly Conference Abstracts, volume 15 of EGU General Assembly Conference Abstracts, page 1571, Apr. 2013.

[115] P. J. Olver. Applications of Lie Groups to Differential Equations, volume 107 of Graduate Texts in Mathematics. Springer-Verlag, 2nd edition, 1993.

[116] M. Onorato, A. R. Osborne, M. Serio, L. Cavaleri, C. Brandini, and C. T. Stansberg. Extreme waves, modulational instability and second order theory: wave flume experiments on irregular waves. European Journal of Mechanics - B/Fluids, 25(5):586 - 601, 2006. Rogue waves European Geosciences Union Assembly. 
[117] B. Parker. The Power of the Sea: Tsunamis, Storm Surges, Rogue Waves, and Our Quest to Predict Disasters. Palgrave Macmillan, 2010.

[118] E. N. Pelinovsky, editor. The Climbing of Tsunami Waves on the Beach. Institute Applied Physics Press, Nizhny Novgorod, 1985.

[119] E. N. Pelinovsky. Tsunami Wave Hydrodynamics. Institute Applied Physics Press, Nizhny Novgorod, 1996.

[120] E. N. Pelinovsky. Hydrodynamics of tsunami waves. In J. Grue and K. Trulsen, editors, Waves in Geophysical Fluids, volume 489 of CISM International Centre for Mechanical Sciences, pages 1-48. Springer Vienna, 2006.

[121] D. H. Peregrine. Surf zone currents. Theoretical and Computational Fluid Dynamics, 10:295-310, 1998.

[122] D. H. Peregrine. Water-wave imapct on walls. Annual Review of Fluid Mechanics, 35(1):23-43, 2003.

[123] D. H. Peregrine and O. Bokhove. Vorticity and surf zone currents. Coastal Engineering Proceedings, 1(26), 2001.

[124] L. J. Pratt. On inertial flow over topography. part 1. semigeostrophic adjustment to an obstacle. Journal of Fluid Mechanics, 131:195-218, 61983.

[125] S. Rhebergen. Discontinuous Galerkin finite element methods for (non)conservative partial differential equations. $\mathrm{PhD}$ thesis, University of Twente, Enschede, February 2010.

[126] S. Rhebergen, O. Bokhove, and J. J. W. van der Vegt. Discontinuous Galerkin finite element methods for hyperbolic nonconservative partial differential equations. Journal of Computational Physics, 227(3):1887 - 1922, 2008.

[127] J. E. Romate. Local error analysis of three-dimensional panel methods in terms of curvilinear surface coordinates. SIAM Journal on Numerical Analysis, 27(2):pp. 529-542, 1990.

[128] J. E. Romate. The numerical simulation of nonlinear gravity waves. Engineering Analysis with Boundary Elements, 7(4):156 - 166, 1990.

[129] J. E. Romate and P. J. Zandbergen. Boundary integral equation formulations for free-surface flow problems in two and three dimensions. Computational Mechanics, 4(4):276-282, 1989.

[130] R. Salmon. Hamiltonian fluid mechanics. Annual Review of Fluid Mechanics, 20(1):225-256, 1988. 
[131] B. Satish, W. D. Gropp, L. C. McInnes, and B. F. Smith. Efficient management of parallelism in object-oriented numerical software libraries. In Modern Software Tools in Scientific Computing, pages 163-202. Birkhauser Press, 1997.

[132] B. Satish, B. Kris, E. Victor, D. G. William, K. Dinesh, G. K. Matthew, L. C. McInnes, B. F. Smith, and Z. Hong. Petsc users manual, 2004. Argonne National Laboratory, NL-95/11 - Revision 2.1.5, http: / /www-unix.mcs.anl.gov/ petsc.

[133] B. Satish, B. Kris, D. G. William, K. Dinesh, G. K. Matthew, L. C. McInnes, B. F. Smith, and Z. Hong. Petsc web page, 2001. http://www.mcs.anl.gov/ petsc.

[134] E. Seas. Design for ship safety in extreme seas. http://www.mar.ist.utl.pt/extremeseas/home.aspx.

[135] L. She Liam. Mathematical modelling of generation and forward propagation of dispersive waves. PhD thesis, University of Twente, Enschede, May 2013.

[136] T. G. Shepherd. A unified theory of available potential energy. Atmosphere-Ocean, 31(1):1-26, 1993.

[137] T. Shimozono, S. Sato, A. Okayasu, Y. Tajima, H. M. Fritz, H. Liu, and T. Takagawa. Propagation and inundation characteristics of the 2011 Tohoku tsunami on the central Sanriku coast. Coastal Engineering Journal, 54(01):1250004, 2012.

[138] C.-W. Shu. A brief survey on discontinuous Galerkin methods in computational fluid dynamics. Advances in Mechanics, 43:541-554, 2013.

[139] Y. Suris. Hamiltonian methods of Runge-Kutta type and their variational interpretation. Matematicheskoe Modelirovanie, 2(4):78-87, 1990.

[140] C. E. Synolakis. The runup of solitary waves. Journal of Fluid Mechanics, 185:523-545, 121987.

[141] A. R. Thornton, A. J. van der Horn, E. Gagarina, W. Zweers, D. van der Meer, and O. Bokhove. Hele-shaw beach creation by breaking waves: a mathematics-inspired experiment. Environmental Fluid Mechanics, pages 1-23, 2014.

[142] W. Tsai and D. K. P. Yue. Computation of nonlinear free-surface flows. Annual Review of Fluid Mechanics, 28(1):249-278, 1996.

[143] M. P. Tulin and M. Landrini. Breaking waves in the ocean and around ships. In N. R. C. Office of Naval Research, Bassin d'Essais des Carenes, editor, Twenty-Third Symposium on Naval Hydrodynamics, pages 713-745. The National Academies Press, 2001. 
[144] J. J. W. van der Vegt and Y. Xu. Space-time discontinuous Galerkin method for nonlinear water waves. Journal of Computational Physics, 224(1):17 - 39, 2007. Special Issue Dedicated to Professor Piet Wesseling on the occasion of his retirement from Delft University of Technology.

[145] J. J. W. v. d. Vegt and S. K. Tomar. Discontinuous Galerkin method for linear free-surface gravity waves. Journal of Scientific Computing, 22-23(1-3):531-567, 2005.

[146] T. Vinje and P. Brevig. Numerical simulation of breaking waves. Advances in Water Resources, 4(2):77 - 82, 1981.

[147] A. I. Vol'pert. The spaces BV ans quasilinear equations. Mathematics of the USSRSbornik, 2(2):225-267, 1967.

[148] S. L. Wakelin. Variational principles and the finite element method for channel flows. PhD thesis, University of Reading, Reading, United Kingdom, 1993.

[149] C. Wang and G. Wu. A brief summary of finite element method applications to nonlinear wave-structure interactions. Journal of Marine Science and Application, 10(2):127-138, 2011.

[150] C. Y. Wang. Exact solutions of the steady-state Navier-Stokes equations. Annual Review of Fluid Mechanics, 23:159-177, 1991.

[151] C. Z. Wang and G. X. Wu. Interactions between fully nonlinear water waves and cylinder arrays in a wave tank. Ocean Engineering, 37(4):400 - 417, 2010.

[152] J.-H. Westhuis. The numerical simulation of nonlinear waves in a hydrodynamic model test basin. PhD thesis, University of Twente, Enschede, 2001.

[153] G. B. Whitham. A general approach to linear and non-linear dispersive waves using a Lagrangian. Journal of Fluid Mechanics, 22:273-283, 61965.

[154] G. B. Whitham. Non-linear dispersion of water waves. Journal of Fluid Mechanics, 27:399-412, 21967.

[155] G. B. Whitham. Linear and Nonlinear Waves. John Wiley \& Sons, 1974.

[156] G. X. Wu and Z. Z. Hu. Simulation of nonlinear interactions between waves and floating bodies through a finite-element-based numerical tank. Proceedings: Mathematical, Physical and Engineering Sciences, 460(2050):pp. 2797-2817, 2004.

[157] H. Yeh, W. Li, and Y. Kodama. Mach reflection and KP solitons in shallow water. European Physical Journal Special Topics, 185:97-111, July 2010.

[158] Z. Yoshida. Clebsch parameterization: Basic properties and remarks on its applications. Journal of Mathematical Physics, 50(11):113101-113101-16., 2009. 
[159] V. E. Zakharov. Stability of periodic waves of finite amplitude on the surface of a deep fluid. Journal of Applied Mechanics and Technical Physics, 9(2):190-194, 1968.

[160] W. Zweers. Soliton-splash webpage with details of the Bore-soliton-splash experiment, youtube-videos and photos, 2010. http://www.woutzweers.nl/ Click on "what's new". Bore-soliton-splash Youtube channel: http://www. youtube.com/user/woutzweers. 
Bibliography 


\section{Acknowledgments}

This dissertation is the outcome of my research performed at the Department of Applied Mathematics at the University of Twente. It is largely inspired and encouraged by my supervisors, family, friends and colleagues whom I would like to acknowledge.

First and foremost, I would like to express my gratitude towards my supervisors: Jaap van der Vegt and Onno Bokhove without whom this thesis would not be possible. I am thankful for the attentive guidance, fruitful discussions and the continuous support they provided. I owe my deepest appreciation to Jaap, whose wisdom and caring attitude have led me through these years. It has been an honor to work with him. I am grateful to Jaap for his patience, interesting discussions and various aspects of mathematics he taught me. I would like to thank Onno for his enthusiasm, inspiration and for his encouragement to improve my work. Under his supervision I learned that one should never give up. His willingness to accomplish the best results and his insightful suggestions played an important role in my $\mathrm{PhD}$.

I would like to thank Prof. Dr. P. M. G. Apers, Prof. Dr. Ir. E. W. C. van Groesen, Prof. Dr. H. J. Zwart, Prof. Dr. Ir. R. H. M. Huijsmans, Prof. Dr. A. E. P. Veldman and Dr. T. Bunnink for being part of my graduation committee and for the time and effort they spent reviewing my thesis. I appreciate our discussions, which provided me with a different perspective on the matters.

Let me thank all the current and former members of the MaCS group for the cooperation, fun and friendship I received. I will keep warm memories about the time here. I am particularly grateful for all the assistance and friendly attitude given by Mikhail Bochev, his wife Lisette and their daughters Maria and Varvara. I am very thankful to the secretaries Marielle, Linda, Carin and Marja who helped me with various issues arising around life in the Netherlands.

I would like to offer my special thanks to Anthony and Shavarsh, whose support, reliability and sense of humor I truly appreciate. It is a pleasure to know them. I would like to thank Irana for all the valuable help (with translation especially) and understanding she offered. I will miss our cookie-afternoons. Let me express my gratitude to Wenny, 
whose patience and loving heart I greatly admire. Many thanks to Lilya and Sveta for being an incredible source of joy. In this wonderful company it is a pleasure to speak about everything in the world.

I am obliged to my group and collaborators Bob, Bram, Deepak, Devashish, Ferenc, Freekjan, Lulu, Sjoerd, Tatyana, Thomas, Tito for being a good team, for the research assistance and interesting seminars. I hope the collaboration does not stop here. I would like to express special gratitude to Sander and Vijaya for the contribution to my research and extensive tutorials. Many thanks to colleagues Anastasia, Bettina, David, Didit, Edo, Edson, Felix, Gijs, Huan, Ivan, Jaroslav, Julia, Maurice, Milos, Mika, Nida, Paolo, Rudi for the nice atmosphere they created at work.

I owe sincere and earnest thankfulness to the clergy and parish of St. Nicholas of Myra Russian Orthodox Church in Amsterdam for giving a hand whenever I needed it. Many thanks to my friends Dasha, Lera, Olga and Marina, whose friendship made the days sunnier. I would like to express great appreciation to my dear friend Masha. The doors of her house are always open to me and her heart is full of love. I am happy to know her for all these years and I am proud to be her friend. Many thanks to my dear cousin Olesya. I admire her optimism and greatly appreciate our life-long friendship.

This thesis would not be possible without my beloved family, mama Nadezhda and grandma Galina. They always trust me, believe in me and share their love with me which is priceless. I am thankful to my late uncle Sergei, who was my earliest tutor of mathematics. My gratitude to the Göttsche family, especially Helga and Peter, for the privilege to be a part of their amiable family.

Finally, I would like to express a special gratitude to my husband Ove for all the wonderful moments we share. Thank you for being there for me. Your love and patience are indispensable. 